\title{
5. Site 356: SAO PAUlo PlateaU
}

\author{
The Shipboard Scientific Party'
}

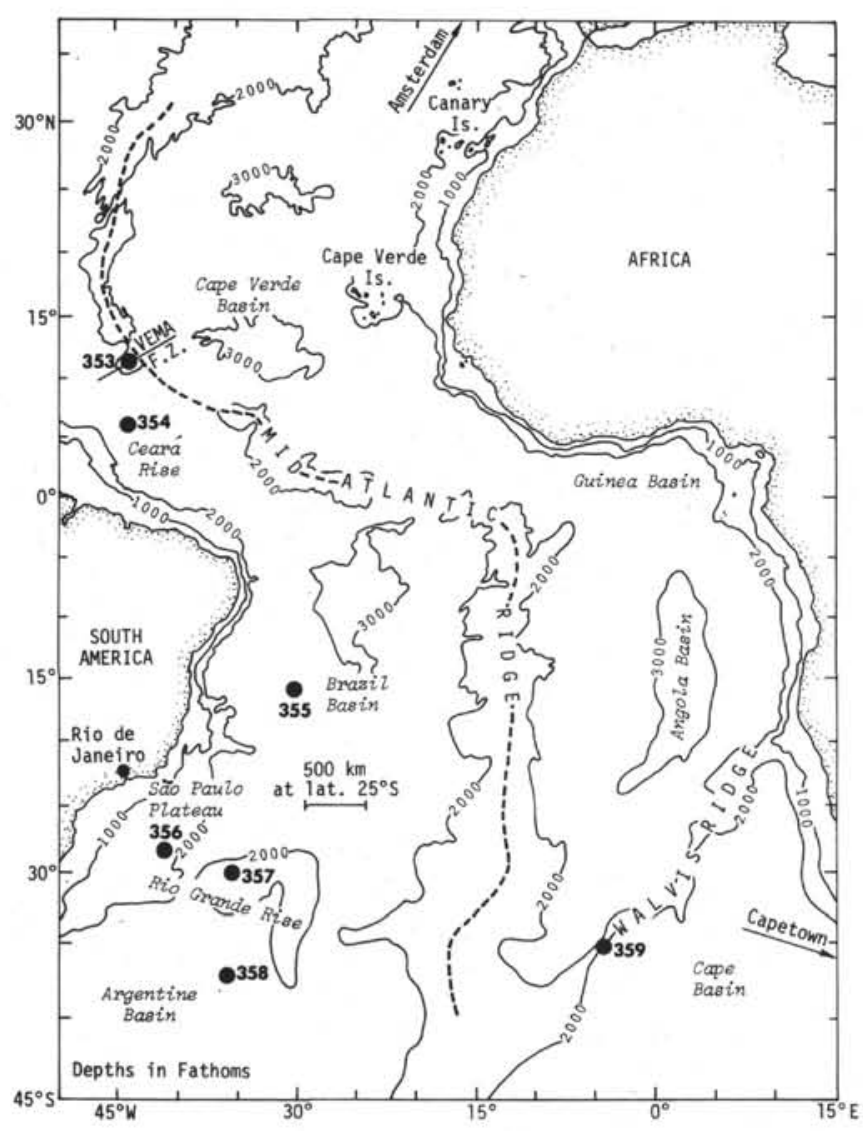

\section{SITE DATA}

Date Occupied: 16 November 1974 (1317Z)

Date Departed: 21 November 1974 (1850Z)

Time on Site: 5 days, 5 hours, 33 minutes

Position: $28^{\circ} 17.22^{\prime} \mathrm{S}, 41^{\circ} 05.28^{\prime} \mathrm{W}$

Accepted Water Depth: 3175 meters (echo sounding, corrected)

Penetration: 741 meters

Number of Holes: 2

'K. Perch-Nielsen, Eidg. Technische Hochschule, Zürich, Switzerland (Co-chief scientist); P.R. Supko, Scripps Institution of Oceanography, La Jolla, California (Co-chief scientist); A. Boersma, Lamont-Doherty Geological Observatory, Palisades, New York; R.L. Carlson, University of Washington, Seattle, Washington; M.G. Dinkelman, The Florida State University, Tallahassee, Florida; R.V. Fodor, University of New Mexico, Albuquerque, New Mexico; N. Kumar, Lamont-Doherty Geological Observatory, Palisades, New York; F. McCoy, Lamont-Doherty Geological Observatory, Palisades, New York; J. Thiede, Oregon State University, Corvallis, Oregon; H. B. Zimmerman, Union College, Schenectady, New York.
Number of Cores:

Hole 356: 44

Hole 356A: 2

Total Length of Cored Sections:

Hole 356: 418 meters

Hole 356A: 19 meters

Total Core Recovered:

Hole 356: 215.9 meters

Hole 356A: 17.6 meters

Principal Results: We cored sediments to a subbottom depth of 741 meters on the southern edge of the São Paulo Plateau. The oldest sediments recovered are Albian laminated dolomitic limestones, which were deposited at intermediate depth $(\sim 1000 \mathrm{~m})$. These are overlain by Turonian-Coniacian conglomerates and mudstones, rich in organic matter, which were laid down in part at least, in a semirestricted basin of local or regional extent. PostConiacian sediments are mostly pelagic biogenic carbonates with varying admixtures of terrigenous material, and were deposited in an open marine basin. The carbonate section is continuous across the CretaceousTertiary boundary; a thick Danian section was recovered. Siliceous components are present in significant quantities only in the Eocene. There is a hiatus at the PaleoceneEocene boundary, an important hiatus from middle

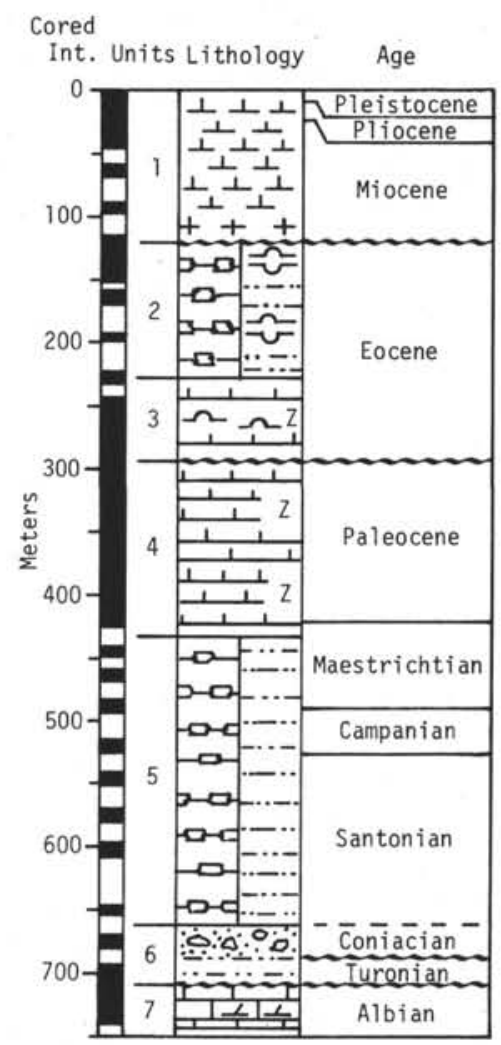


Eocene to lower Miocene, and a hiatus from lower Miocene to Pliocene.

\section{BACKGROUND AND OBJECTIVES}

Site 356 is on the southeastern edge of the São Paulo Plateau at a water depth of 3175 meters (Figure 1). The plateau is triangular in plan view, and extends up to 950 $\mathrm{km}$ offshore from the Brazilian shoreline.

Two-ship refraction profiles in 1960 and seismicrefraction surveys in 1967, carried out by LamontDoherty Geological Observatory, had indicated in this area a shallow seismic horizon which could contain evaporites (Leyden et al., 1971). As a result of further surveys carried out by L-DGO in 1972, 1973, and 1974, the full extent of the diapir zone off the margin of Brazil has been established (Leyden and Nunes, 1972; Leyden et al., in press). The diapir zone off Brazil extends 810 $\mathrm{km}$ offshore at $28^{\circ} \mathrm{S}$ and narrows to less than $30 \mathrm{~km}$ at $11^{\circ} \mathrm{S}$. In the south, the seaward edge of the diapir zone lies in more than 2000 meters of water, whereas in the north, the zone occurs only on the shelf (Figure 2).

Most of the area of the São Paulo Plateau is underlain by diapirs. An east-west basement ridge marks the southern margin of the plateau (Figure 3). This ridge can be followed westward for some distance in the sub-bottom and may connect on land with the southern edges of the Precambrian Ponta Grossa Arch (see Kumar et al., this volume).

The eastern edge of the São Paulo Plateau roughly coincides with the eastern limit of the diapir zone; whether the scarp is controlled by basement structure is not known.
A similar zone of diapirs has been mapped between $1^{\circ} \mathrm{N}$ and $14^{\circ} \mathrm{S}$ from Gabon to Angola, off the West African margin (Leyden et al., 1972; Pautot et al., 1972). Leyden and Nunes (1972) have shown that when the boundaries of the diapir zones on both sides of the Atlantic are brought together, they fit each other perfectly and form one continuous zone of salt deposition in the South Atlantic.

The salt present on both sides of the Atlantic is supposed to be Aptian-Albian in age (Asmus and Ponte, 1973). If the rifting in the South Atlantic started sometime in the Neocomian, about 127 m.y. ago (Larson and Ladd, 1973), the South Atlantic must have still been a narrow restricted basin in Aptian-Albian time. Spreading started in the southern South Atlantic, and the region south of Walvis Ridge and Rio Grande Rise formed an open marine basin. The Walvis Ridge and the basement ridge of the São Paulo Plateau, together with the Rio Grande Rise, formed a barrier to open circulation. Circulation was also restricted between the North Atlantic and the South Atlantic until late in the Cretaceous (Reyment and Tait, 1972). Hence, conditions were well suited for salt deposition in the northern South Atlantic.

Site 356 is in the zone between the escarpment of São Paulo Plateau and the area of diapirs (Figure 2). This zone either contains a very thin salt layer (not thick enough to form large diapirs), gypsum, and anhydrite, or contains nonevaporitic sediments. Owing to considerations of safety and politics, it was not possible to drill within the zone of diapirs (most of the diapir zone lies within the 200 -mile limit claimed by Brazil as

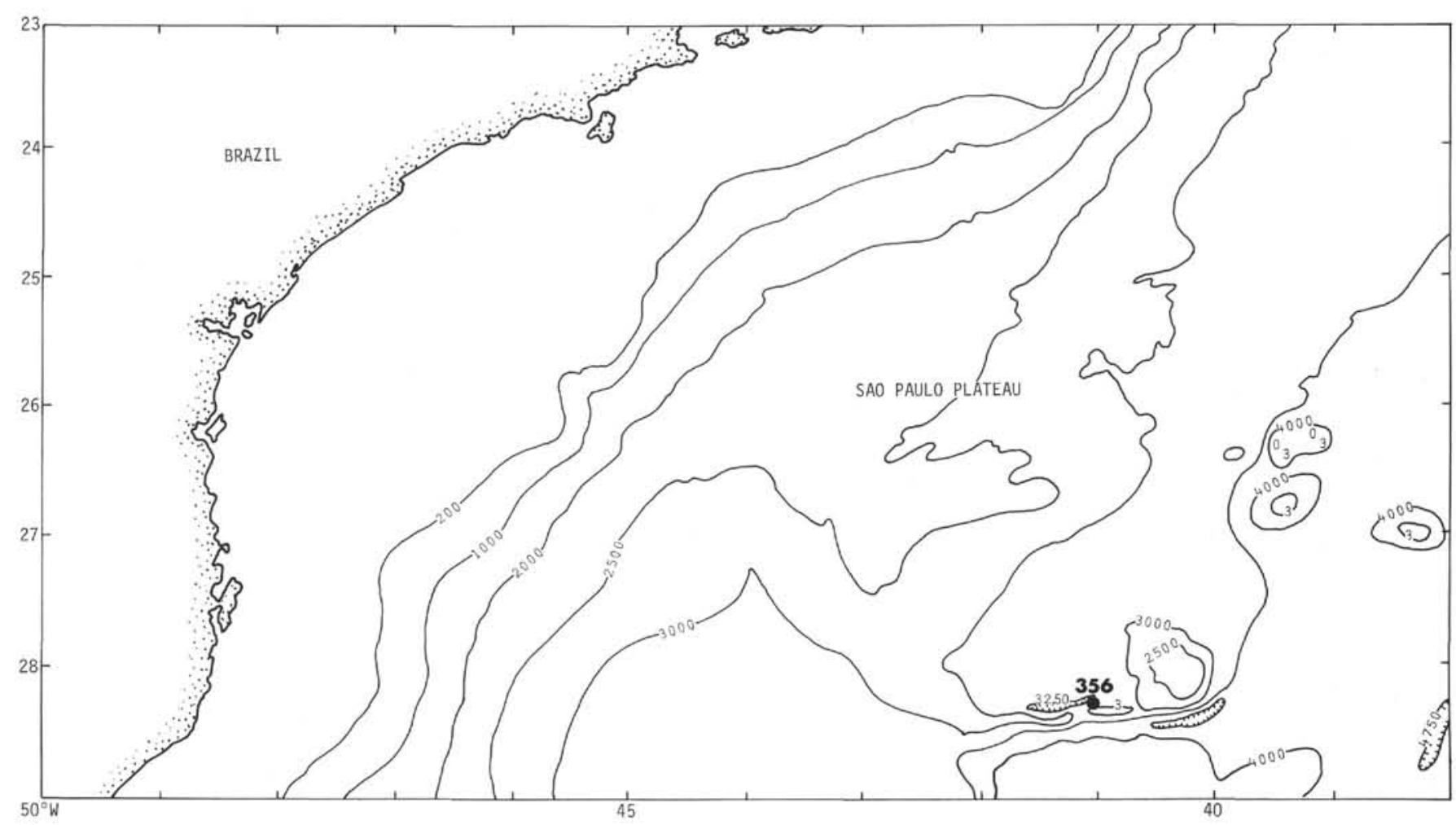

Figure 1. Base map of São Paulo Plateau showing location of Site 356. Contours in meters, from Connary and Moody, 1975, bathymetry of the continental margin of Brazil (unpublished). 


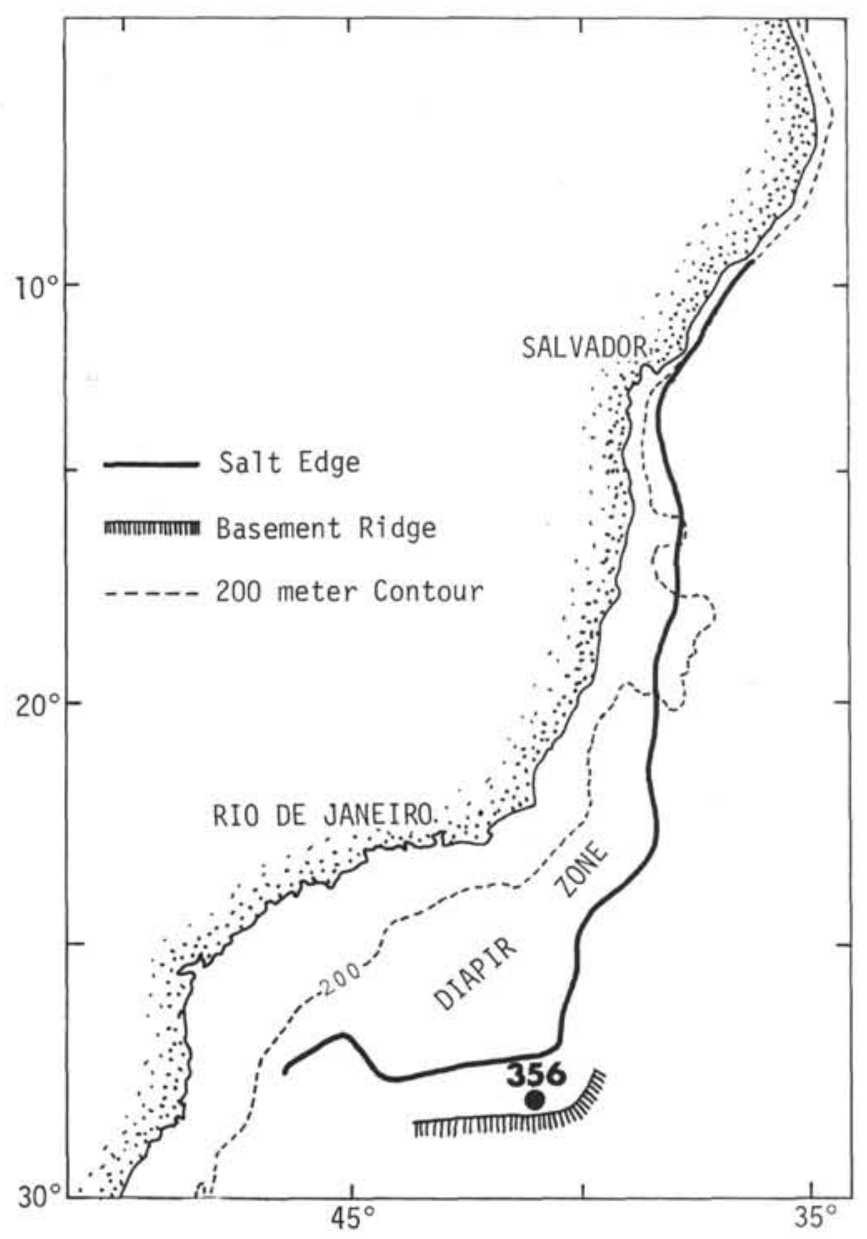

Figure 2. Site 356 in relation to the shelf break, inferred edge of the salt diapir zone, and southern escarpment of the São Paulo Plateau. From Leyden et al (in press).

territorial waters). The present site was chosen because it appeared to offer us the opportunity to sample (a) sediments younger than the salt, (b) sediment equivalent in age to salt, (c) sediments older than the salt, and (d) the basement of the São Paulo Plateau.

Drilling at Site 356 was expected to help answer the following questions:

1) What was the nature of the South Atlantic basin before the time salt was deposited-i.e., shallow or deep?

2) Did the salt form in deep or shallow water?

3) What is the nature of the basement rocks underlying the salt: is the basement ridge forming the southern edge of the São Paulo Plateau made up of oceanic tholeiites, or rocks related to fracture zones?

4) What is the relationship between the basement under the São Paulo Plateau and the basement under the Rio Grande Rise?

Site 356 was the first site drilled to address the question of the origin of salt in the South Atlantic. All pre-existing information on salt came from onshore outcrops and wells, some wells on the shelf, and seismic data. In addition to investigating the salt question, we attempted, by coring in the upper part of the stratigraphic sequence, to detail changes in the pattern of sediment accumulation on the plateau during the Tertiary. The sediments were expected to show transition from an isolated basin in the Cretaceous to a semi-isolated enclosed condition, and finally to open marine conditions. Periods of eustatic low sea level, such as the Miocene and Plio-Pleistocene, were expected to show increased supply of terrigenous components.

\section{OPERATIONS}

We approached Site 356 on a heading of $210^{\circ} \mathrm{T}$. It was our intent to come onto a course of $180^{\circ}$, roughly at right angles to the basement ridge, some eight miles north (point A, Figure 4) of the proposed site location (see Figure 3, $2150 \mathrm{hr}$ on LDGO record number 1514, cruise RC 16). At 1020Z, 16 November 1974, we believed we were about 4.5 miles north of point $\mathrm{A}$ (based on a computer input omission and resultant bad dead reckoning), and altered course to $180^{\circ} \mathrm{T}$. A satellite fix received just before the turn showed Glomar Challenger east and slightly north of the turn point. We changed heading to $230^{\circ}$ at $1045 \mathrm{Z}$ and to $180^{\circ}$ at $1053 \mathrm{Z}$ and reduced speed to 6 knots at $1100 \mathrm{Z}$. We passed the proposed site and found a more likely drilling location at $1200 \mathrm{Z}$. At $1233 \mathrm{Z}$ we turned onto a reciprocal course. We dropped the beacon while underway at $1317 \mathrm{Z}$, retrieved gear, and returned over the beacon.

The site is on the north slope of the basement ridge (i.e., south of the valley), with $\sim 0.75 \mathrm{sec}$ to basement (Figure 5) and roughly the same sequence of internal reflectors as the (slightly thicker) section north of the valley (Figure 4).

As at all Leg 39 sites, the bottom-hole assembly consisted of a Smith 10-1/8" F94CK chisel-tooth tungsten carbide inset bit, core barrel, three 8-1/4" drill collars, one Baash-Ross bumper sub, three 8-1/4" drill collars, two Baash-Ross bumper subs, two 8-1/4" drill collars, one $7-1 / 2^{\prime \prime}$ drill collar, and one joint of $5-1 / 2^{\prime \prime}$ heavy weight drill pipe. The bit chosen seems to offer the best compromise between penetration rates in soft sediments and ability to drill through hard formations such as chert or basalt.

Accepted water depth as measured by PDR was 3175 meters, corrected, or 3185 meters to the rig floor. The driller thought he felt bottom in the joint between 3193.5-3203 meters; a water core was pulled. The next attempt (3203-3212.5 m) yielded about a thumbnail full of Pleistocene foraminifer sand. Since bottom had obviously been reached somewhere in this joint, depth to the sediment/water interface was accepted as 3203 meters below the rig floor for measurement purposes, and coring began.

The first nine cores (see Table 1) cut very easily and quickly (5-15 $\mathrm{min}$ per core). The sequence between Cores 9 and 16 was silicified, and it took about twice as long to cut each core (Figure 6). (At about this degree of induration, it is no longer possible to pump the material completely away in the drilled intervals; but the problem did not arise in this case, since this section was of high biostratigraphic interest and therefore continuously cored.) The section from Cores 19 through 30 cut at a slightly faster rate; the downhole 


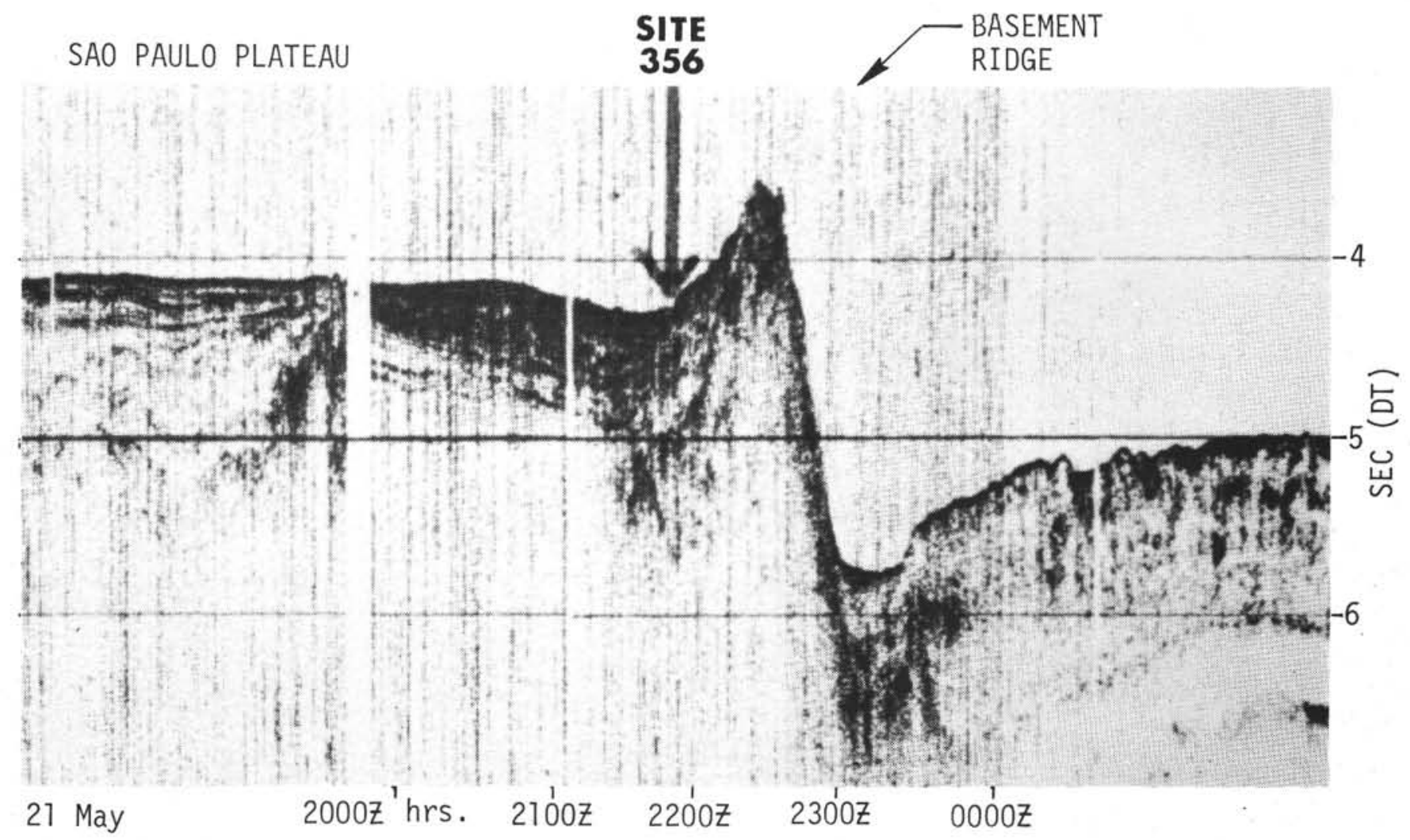

Figure 3. Lamont-Doherty Geological Observatory seismic profile number 1514 (Cruise RC 16-10) shot on a heading of $153^{\circ}$ across the location of Site 356 and the basement ridge marking the southern edge of the São Paulo Plateau.

TABLE 1

Coring Summary, Site 356

\begin{tabular}{|c|c|c|c|c|}
\hline Core & $\begin{array}{l}\text { Depth Below } \\
\text { Sea Floor } \\
(\mathrm{m})\end{array}$ & $\begin{array}{l}\text { Length } \\
\text { Cored } \\
\text { (m) }\end{array}$ & $\begin{array}{l}\text { Length } \\
\text { Recovered } \\
\text { (m) }\end{array}$ & $\begin{array}{l}\text { Recovery } \\
\text { (m) }\end{array}$ \\
\hline \multicolumn{5}{|c|}{ Hole 356} \\
\hline 1 & $0.0-9.5$ & 9.5 & $\operatorname{Tr}$ & 0 \\
\hline 2 & $9.5-19.0$ & 9.5 & 9.5 & 100 \\
\hline 3 & $38.0-47.5$ & 9.5 & 9.5 & 100 \\
\hline 4 & $57.0-66.5$ & 9.5 & 9.4 & 99 \\
\hline 5 & $85.5-95.0$ & 9.5 & 9.5 & 100 \\
\hline 6 & $114.0-123.5$ & 9.5 & 8.8 & 93 \\
\hline 7 & $133.0-142.5$ & 9.5 & 9.5 & 100 \\
\hline 8 & $161.5-171.0$ & 9.5 & 2.6 & 27 \\
\hline 9 & $190.0-199.5$ & 9.5 & 2.5 & 26 \\
\hline 10 & $218.5-228.0$ & 9.5 & 5.2 & 55 \\
\hline 11 & $237.5-247.0$ & 9.5 & 4.7 & 50 \\
\hline 12 & $247.0-256.5$ & 9.5 & 3.2 & 34 \\
\hline 13 & $256.5-266.0$ & 9.5 & 1.8 & 19 \\
\hline 14 & $266.0-275.5$ & 9.5 & 1.6 & 17 \\
\hline 15 & $275.5-285.0$ & 9.5 & 4.5 & 47 \\
\hline 16 & $285.0-294.5$ & 9.5 & 2.1 & 22 \\
\hline 17 & $294.5-304.0$ & 9.5 & 9.5 & 100 \\
\hline 18 & $304.0-313.5$ & 9.5 & 8.6 & 91 \\
\hline 19 & $313.5-323.0$ & 9.5 & 8.3 & 87 \\
\hline 20 & $323.0-332.5$ & 9.5 & 4.6 & 48 \\
\hline 21 & $332.5-342.0$ & 9.5 & 5.2 & 55 \\
\hline 22 & $342.0-351.5$ & 9.5 & 8.3 & 87 \\
\hline 23 & $351.5-361.0$ & 9.5 & 8.4 & 88 \\
\hline 24 & $361.0-370.7$ & 9.5 & 9.5 & 100 \\
\hline 25 & $370.5-380.0$ & 9.5 & 6.3 & 66 \\
\hline 26 & $380.0-389.5$ & 9.5 & 9.5 & 100 \\
\hline 27 & $389.5-399.0$ & 9.5 & 9.3 & 98 \\
\hline 28 & $399.0-408.5$ & 9.5 & 8.8 & 93 \\
\hline
\end{tabular}

TABLE 1 - Continued

\begin{tabular}{ccccc}
\hline Core & $\begin{array}{c}\text { Depth Below } \\
\text { Sea Floor } \\
(\mathrm{m})\end{array}$ & $\begin{array}{c}\text { Length } \\
\text { Cored } \\
(\mathrm{m})\end{array}$ & $\begin{array}{c}\text { Length } \\
\text { Recovered } \\
(\mathrm{m})\end{array}$ & $\begin{array}{c}\text { Recovery } \\
(\mathrm{m})\end{array}$ \\
\hline 29 & $408.5-418.0$ & 9.5 & 9.2 & 97 \\
30 & $418.0-427.5$ & 9.5 & 9.2 & 97 \\
31 & $437.0-446.5$ & 9.5 & 9.5 & 100 \\
32 & $456.0-465.5$ & 9.5 & 8.1 & 85 \\
33 & $484.5-494.0$ & 9.5 & 9.5 & 100 \\
34 & $513.0-522.5$ & 9.5 & 9.5 & 100 \\
35 & $541.5-551.0$ & 9.5 & 9.5 & 100 \\
36 & $570.0-579.5$ & 9.5 & 6.0 & 63 \\
37 & $598.5-608.0$ & 9.5 & 9.5 & 100 \\
38 & $646.0-655.5$ & 9.5 & 4.2 & 44 \\
39 & $674.5-684.0$ & 9.5 & 8.2 & 86 \\
40 & $693.5-703.0$ & 9.5 & 9.5 & 100 \\
41 & $703.0-712.5$ & 9.5 & 6.5 & 68 \\
42 & $712.5-722.0$ & 9.5 & 9.0 & 95 \\
43 & $722.0-731.5$ & 9.5 & 9.5 & 100 \\
44 & $731.5-741.0$ & 9.5 & 8.3 & 87 \\
Total & & 418.0 & 315.9 & 76 \\
& & & & \\
Hole 356A & & & & 100 \\
1 & $19.0-28.5$ & 9.5 & 9.5 & 85 \\
2 & $28.5-38.0$ & 9.5 & 8.1 & 93 \\
Total & & 19.0 & 17.6 & \\
\hline & & & & \\
\hline
\end{tabular}

slowing within the Core 19 to 30 interval (Figure 6) was probably a consequence of greater compaction and a higher state of diagenesis of the chalks down the section. Between Cores 30 and 38 , drilling and coring were slower, probably because of the high clay content 


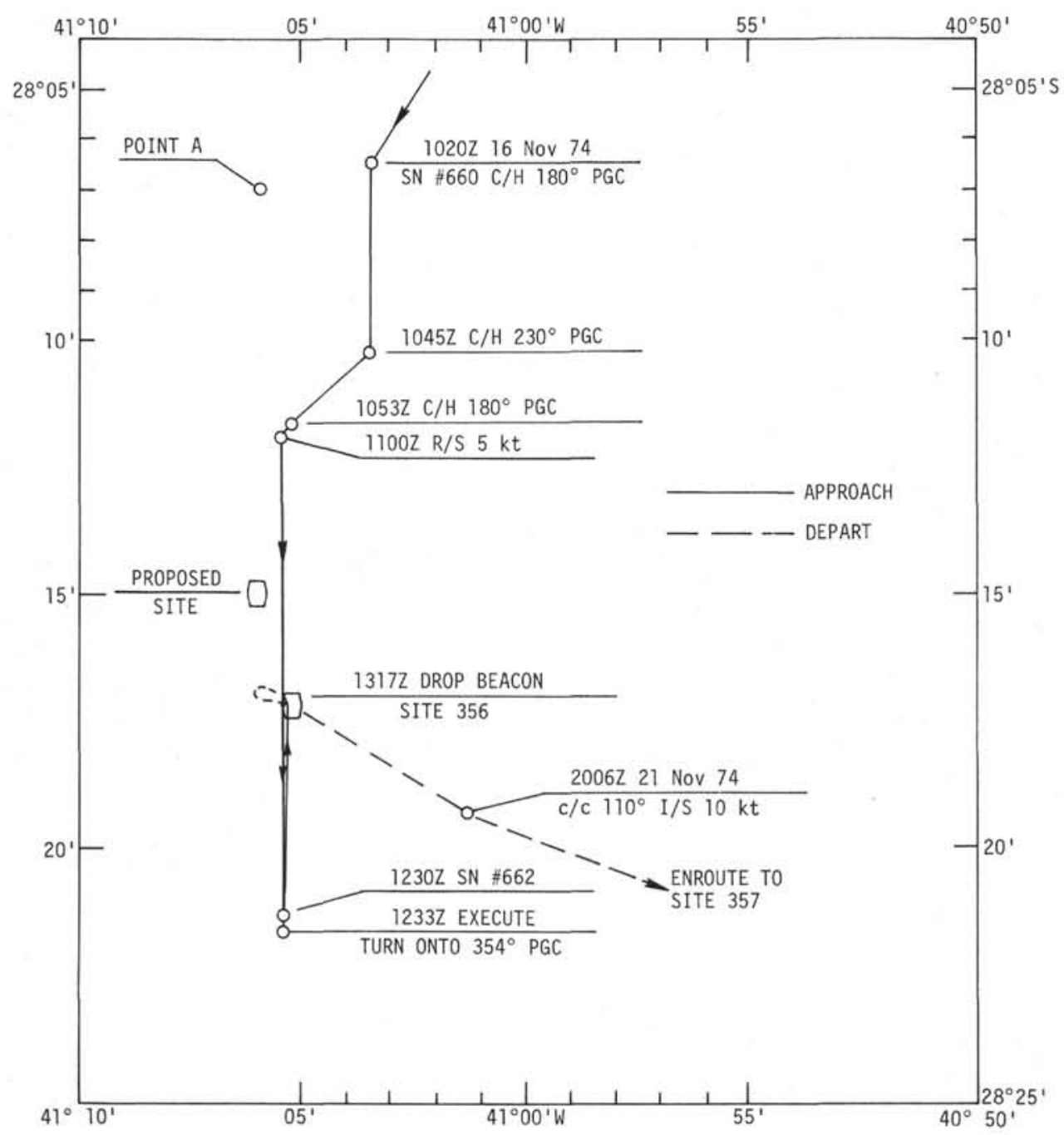

Figure 4. Glomar Challenger track in the vicinity of Site 356, São Paulo Plateau.

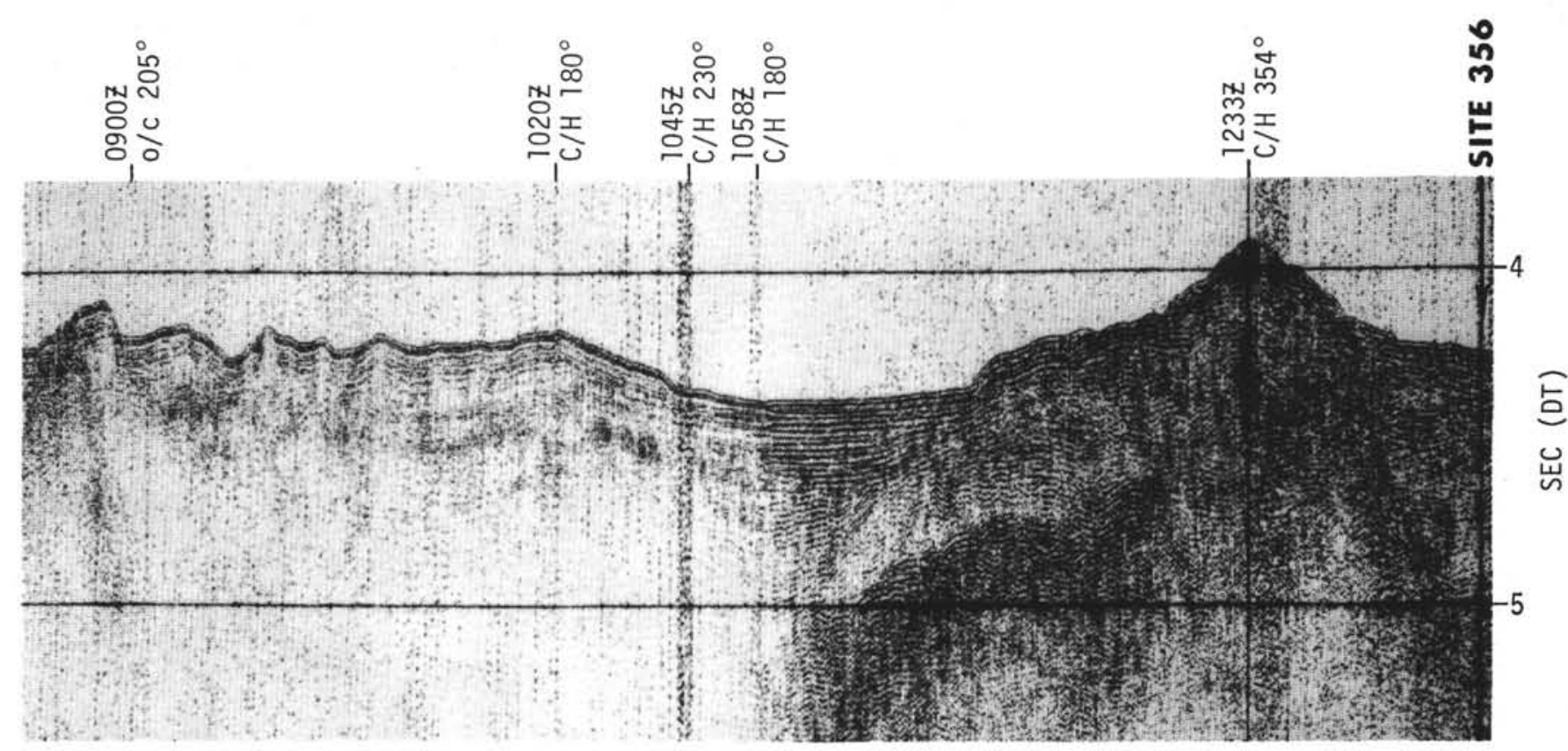

Figure 5. Glomar Challenger seismic reflection profile shot on approach to Site 356. 


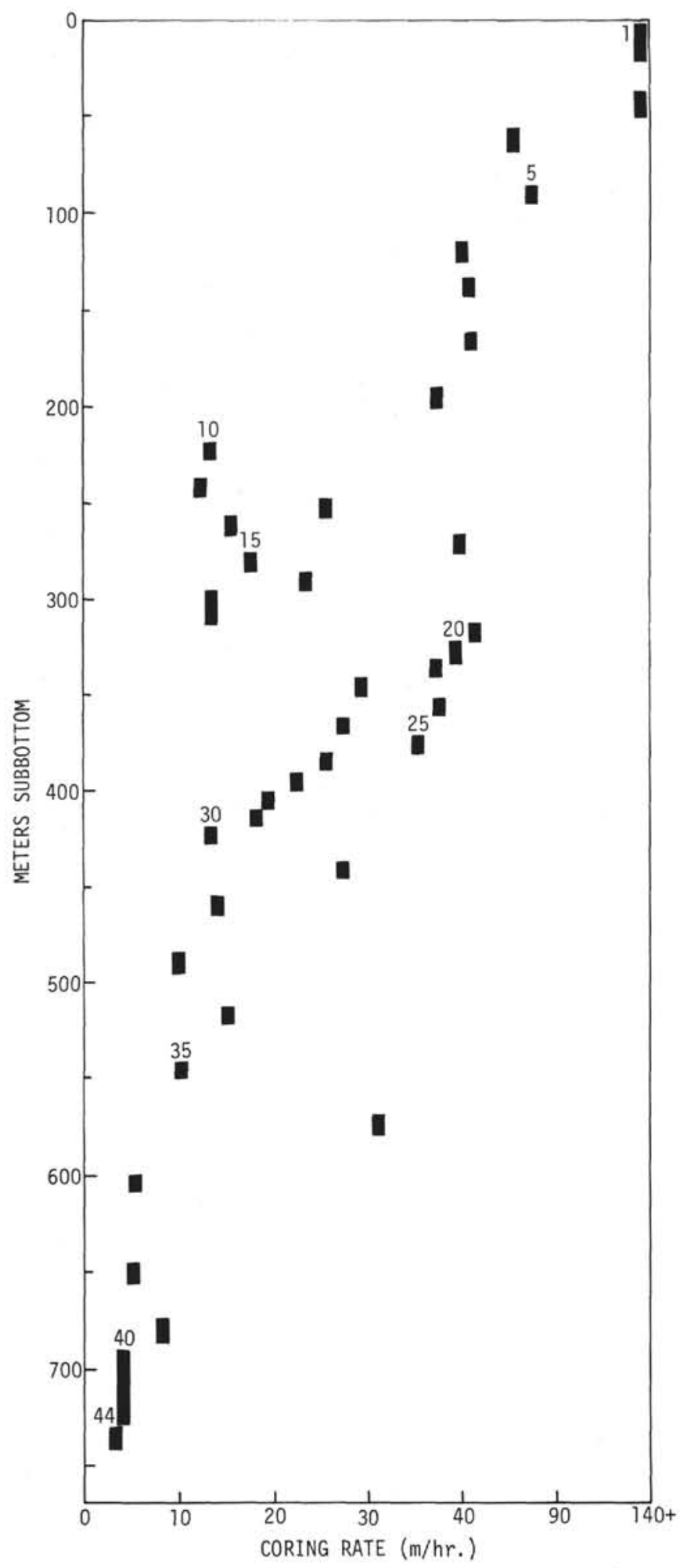

Figure 6. Coring rates, Site 356. Every fifth core is designated by number.

in this marly chalk sequence. The tungsten carbide inset center bit was dropped to drill the interval between 608 and 646 meters subbottom, at a rate of about one joint per hour.

The limestone section below Core 38 cored very slowly; it took up to three hours to cut a single core.
This rate $(3 \mathrm{~m} / \mathrm{hr})$ is of the same order as that required to core basalt, and presents a real problem in penetrating such a limestone section when time is a constraining factor.

Basement at $0.75 \mathrm{sec}$ on the approach (Figure 4) and departure records correlates with the top of the limestone, but there is some faint indication of layering for an additional $0.25 \mathrm{sec}$, which, assuming a velocity of $2.4 \mathrm{~km} / \mathrm{sec}$, could be an additional 300 meters of sedimentary rock above the igneous basement of the ridge. At a rate of three hours per core, an additional four days would have been needed to core to basement. Accordingly, we decided to abandon the site after cutting Core 44.

The pipe was tripped to slightly above the mud line and a second hole was spudded at $0650 \mathrm{hr}, 21$ November 74 , in order to sample the uncored interval between 19 and 38 meters subbottom, which should contain a hiatus or very slow rate of deposition. Core $1 \mathrm{~A}$ was cut and retrieved, Core $2 \mathrm{~A}$ was cut and tripped to the surface with the drill pipe. Examination of Core 1A showed it to correlate almost exactly with Core 3, indicating we were about 18 meters too low in section. There are two possible explanations for the depth discrepancy: We may have spudded Hole $356 \mathrm{~A}$ at a point some 18 meters shallower, or the drill pipe count may have been in error by two joints. The second explanation is the more probable, since the ship had not been offset to spud Hole 356A, and color changes and an ash layer in Cores 3 and $1 \mathrm{~A}$ correlate so closely. By the time the situation was discovered, the pipe had been tripped almost to the surface. The Chief Scientists awarded themselves the Royal Order of Albatross and hindsight wins again!

Glomar Challenger got underway at 1850Z, 21 November 74 , on course $290^{\circ}$; streamed gear, and executed a Williamson turn to pass over the beacon on course $120^{\circ}$ at 6 knots. We crossed the beacon at $1935 \mathrm{Z}$, and at 2006 altered course to $110^{\circ}$ and increased speed to 10 knots, enroute to Site 357 .

\section{LITHOLOGY}

Site 356 was drilled at the southeastern edge of São Paulo Plateau on the Brazilian continental margin. The total penetration was 741 meters and the oldest sediments drilled were of late Albian age. Crystalline basement was not reached. The sequence consists of calcareous, calcareous hemipelagic, pelagic siliceouscalcareous, and terrigenous sediments. The sequence has been divided into the seven units described below. Table 2 and Figure 7 summarize the lithology and stratigraphy of the sedimentary section drilled at Site 356.

\section{Unit 1}

Unit 1 starts with Core 1 and ends in Core 5, Section 5. It is composed primarily of calcareous oozes. A 1.5 meter layer of siliceous nannofossil ooze occurs within the unit, and the bottom of the unit is marly in composition. Colors range from grayish orange to greenish gray to pinkish gray. Texturally the unit is composed of $55 \%$ clay-, $25 \%$ silt-, and $20 \%$ sand-sized 
TABLE 2

Lithologic Summary, Site 356

\begin{tabular}{|c|c|c|c|c|c|}
\hline Unit & Cores & $\begin{array}{l}\text { Dep th Below } \\
\text { Sea Floor } \\
\text { (m) }\end{array}$ & $\begin{array}{l}\text { Thickness } \\
(\mathrm{m})\end{array}$ & Age & Description \\
\hline 1 & 1 to $5(5)$ & $0-93$ & 93 & $\begin{array}{l}\text { Pleistocene- } \\
\text { early Miocene }\end{array}$ & Calcareous oozes \\
\hline 2 & $5(5)$ to $10(4)$ & $93-222$ & 129 & $\begin{array}{l}\text { Middle Eocene- } \\
\text { early Eocene }\end{array}$ & $\begin{array}{l}\text { Calcareous-siliceous and } \\
\text { siliceous-calcareous oozes }\end{array}$ \\
\hline 3 & $10(4)$ to 16 & $222-294.5$ & 72.5 & Early Eocene & Silicified calcareous chalks \\
\hline 4 & 17 to 30 & $294.5-432$ & 137.5 & $\begin{array}{l}\text { Late Paleocene- } \\
\text { Maestrichtian }\end{array}$ & $\begin{array}{l}\text { Nanno and nanno-foram } \\
\text { chalks }\end{array}$ \\
\hline 5 & 31 to 38 & $432-665$ & 233 & $\begin{array}{l}\text { Maestrich tian- } \\
\text { Santonian }\end{array}$ & Marly calcareous chalks \\
\hline 6 & 39 to $41(4)$ & $665-708$ & 43 & $\begin{array}{l}\text { Coniacian- } \\
\text { Mid Turonian }\end{array}$ & $\begin{array}{l}\text { Dark gray mudstones and } \\
\text { clay-pebble conglomerates }\end{array}$ \\
\hline 7 & $41(4)$ to 44 & $708-?$ & $3-?$ & Late Albian & $\begin{array}{l}\text { Marly dolomitic and } \\
\text { calcareous mudstones }\end{array}$ \\
\hline
\end{tabular}

material, and contains $40 \%$ nannofossils, $15 \%$ foraminifers, and $10 \%$ authigenic carbonate. The remaining $35 \%$ consists of clay minerals, pyrite, glauconite, quartz, feldspar, and zeolite. Volcanic glass is present as a discrete ash layer and disseminated in the surrounding sediment.

The 3-cm-thick lower Miocene rhyolitic vitric ash present in Section 4-5 is the only distinct volcanic ash bed observed at this site. Chemical analysis is given in Table 3. Volcanic components are predominantly glass shards (99\%-100\%); crystal components (mica) are rare (1\%); lithic fragments are absent. Three types of shards are present: clear plates, elongated striated fragments, and shards with bubble-wall textures. The majority are colorless and unaltered; about $10 \%$ indicate devitrification. Many of the clear plates have a very fine scalloped surface, suggesting a small-scale bubble-wall texture. Elongated striated shard types contain pipestem vesicles filled with carbonates and clays.

The ash deposit is graded; sand to silt ratios, based upon point counts of smear slides (Figure 8), vary from 1.4 (base) to 1.0 (middle) to 0.8 (top). Glass shard morphological types reflect this size grading: clear

TABLE 3

Chemical Analysis of Volcanic Ash ${ }^{\text {a }}$, Sample 4-5, $58 \mathrm{~cm}$

\begin{tabular}{lc}
\hline $\mathrm{SiO}_{2}$ & 72.8 \\
$\mathrm{TiO}_{2}$ & 0.18 \\
$\mathrm{Al}_{2} \mathrm{O}_{3}$ & 11.9 \\
$\mathrm{FeO}$ & 1.1 \\
$\mathrm{MnO}$ & 0.02 \\
$\mathrm{MgO}$ & 0.06 \\
$\mathrm{CaO}$ & 0.70 \\
$\mathrm{Na}_{2} \mathrm{O}$ & 3.2 \\
$\mathrm{~K}_{2} \mathrm{O}$ & 3.8 \\
$\mathrm{P}_{2} \mathrm{O}_{5}$ & 0.03 \\
& $93.79^{\mathrm{b}}$ \\
\hline
\end{tabular}

\footnotetext{
${ }^{\mathrm{a}} \mathrm{R}$. Fodor, analyst; analysis by electron microprobe.

$\mathrm{b}_{6 \%} \mathrm{H}_{2} \mathrm{O}$ suggested.
}

plates form larger sand-sized fragments, striated forms occur in both sand and silt sizes, and bubble-wall shard fragments occur predominantly in the silt sizes. This graded texture, and its relationship to shard types, as well as the coarseness and angularity of shard particles, all indicate an air-fall deposit with a minimum of disturbance by oceanic currents or by benthic organisms.

In its upper part, Unit 1 also contains slumped zeolitic foraminifer-nannofossil ooze of late Eocene age. The slumped material also includes small pieces of manganese crust.

\section{Unit 2}

Unit 2 begins in Core 5, Section 5, and extends down to Core 10 , Section 4 . It is greenish gray to light greenish gray, and is composed primarily of calcareoussiliceous and siliceous-calcareous oozes. Occasionally the sediment has been compacted enough to be designated as a chalk instead of an ooze. The average textural composition of the unit is $55 \%$ clay, $25 \%$ silt, and $15 \%$ sand. The average composition for the entire unit is as follows: $25 \%$ nannofossils, $10 \%-15 \%$ foraminifers, $10 \%$ radiolarians, and $10 \%$ diatoms. Authigenic components make up $20 \%$, and the remaining $20 \%$ consists of quartz and clay minerals. A few dark gray layers, composed of predominantly siliceous biogenic material, are present.

The unit is quite mottled, and a few distinct burrows are evident. Bedding is seldom apparent. This unit is distinguished from Unit 1 on the basis of its color and the presence of siliceous biogenic components.

\section{Unit 3}

Unit 3 begins in Core 10, Section 4, and extends down to Core 16 . It is composed primarily of silicified chalks, but occasionally contains siliceous-nannofossil oozes, zeolitic chalks, and nannofossil oozes as well. The chalks are well indurated; some of them could almost be termed limestones. One 3-meter layer of marly chalk is present. The unit is light greenish-gray, and sometimes medium bluish gray. It contains $45 \%$ clay-, $30 \%$ silt-, and $25 \%$ sand-sized material, and is 


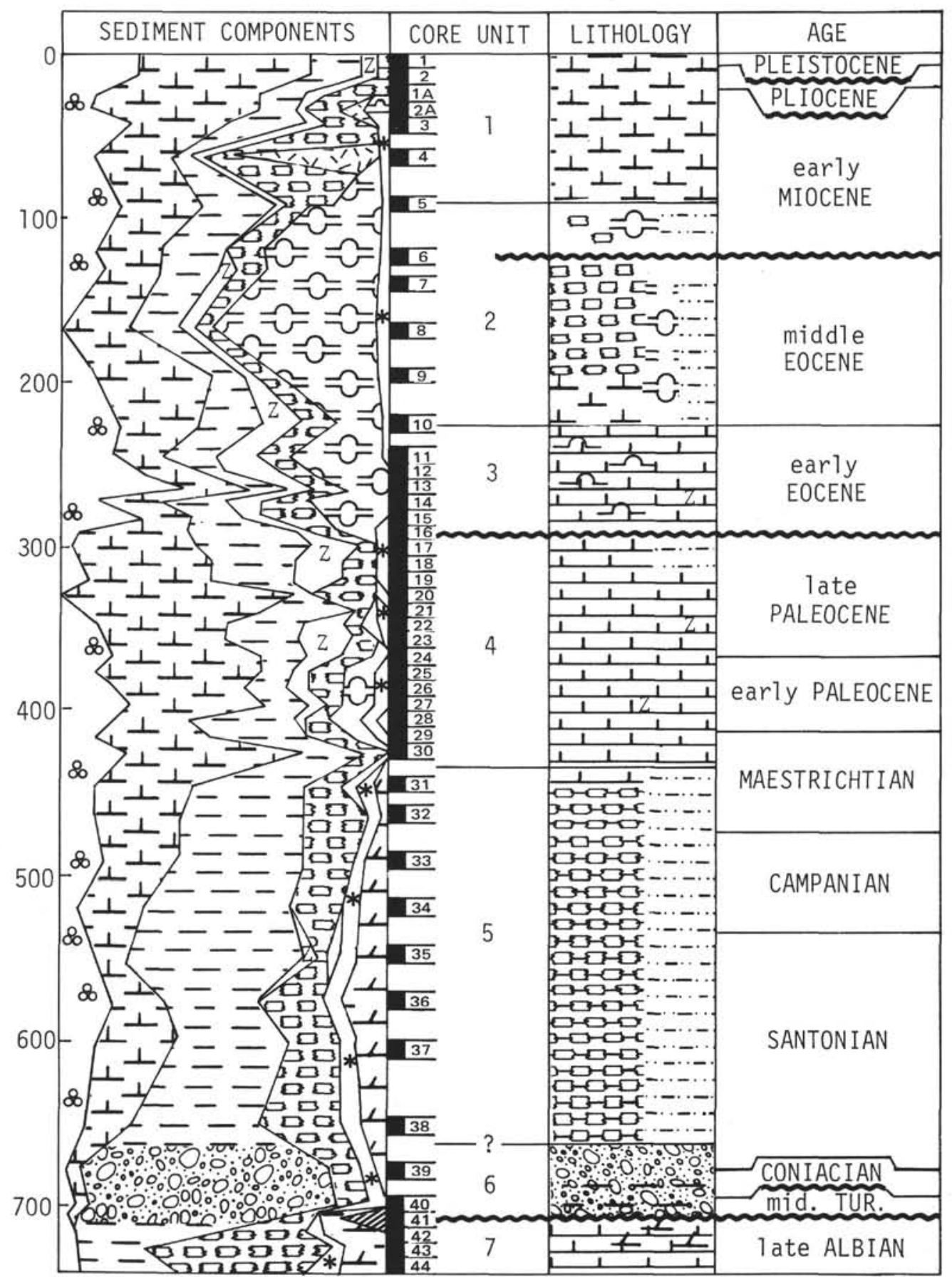

$$
\begin{array}{cccccc}
1 & 1 & 1 & 1 & 1 & 1 \\
0 & 10 & 20 & 30 & 40 & 50
\end{array}
$$

$800-$

$\$$ \&Foraminifers

$L^{\perp}$ Nannofossils

- Clay(clay minerals, quartz

- - feldspar \& mica)

$\mathrm{B}$ 믐 $\mathrm{CO}_{3}$ fragments
$=2$ Diatom-rads

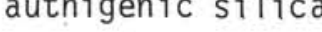

益乲Volc. glass

Z Z Z Zeolites

* * Fe oxides +

Figure 7. Lithologic summary, Site 356. 


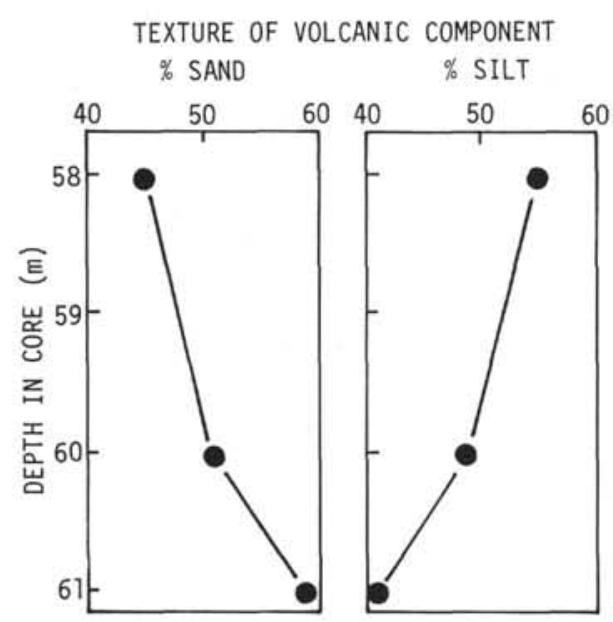

GLASS SHARD MORPHOLOGY

$\%$ CLEAR

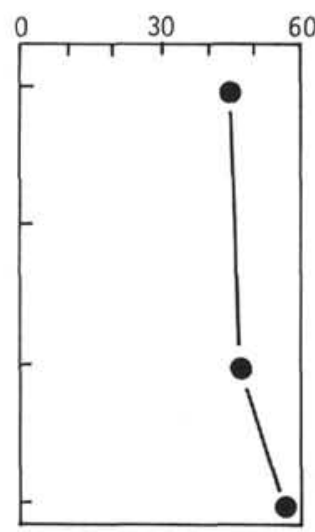
$\%$ STRIATED

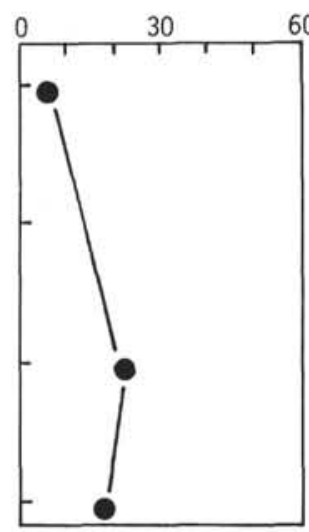

$\%$ BUBBLE-WALL

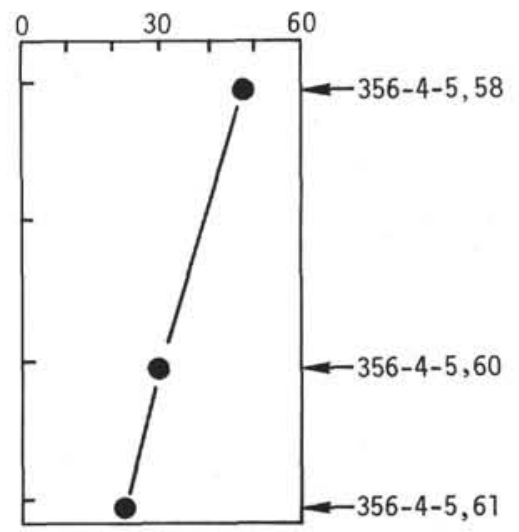

Figure 8. Textural and compositional variations in a volcanic ash layer (Sample 356-4-5, 58-6l cm).

composed of $10 \%$ nannofossils and $10 \%-15 \%$ foraminifers. Authigenic silica (cement for the sediment particles) makes up $15 \%-20 \%$ of the unit and authigenic carbonate $10 \%-15 \%$. The remaining $30 \%$ comprises zeolites and clays, micronodules, and dolomite rhombs.

Unit 3 is basically a well-indurated cemented version of Unit 2. In fact, the main distinction between Units 3 and 2 is the induration of Unit 3, which contains alternating bands of chalks (from $10 \mathrm{~cm}$ to $1 \mathrm{~m}$ thick) and oozes (up to $1 \mathrm{~m}$ thick). Light bluish gray and light greenish gray beds alternate in the chalks. Occasional white streaks (1-2 mm thick) of dolomitic foraminiferal limestone are present within the chalks.

This unit is well bedded and moderately burrowed. Some burrows are moderate yellowish brown with a dark rim, others are dark gray. Burrows reach a maximum width of $1 \mathrm{~cm}$ in this unit.

\section{Unit 4}

This unit extends from Core 17 to Core 30 . Unit 4 is distinguished from the overlying Unit 3 by a lack of siliceous material, and is composed of nannofossil and nannofossil-foraminifer chalks. Sometimes the composition grades to marly nannofossil chalk and zeolitic nannofossil chalk. The terrigenous component increases toward the base of the unit. Cores 28 and 29 contain several 10 - to $40-\mathrm{cm}$-thick layers of ferruginous calcareous mudstone. Colors in this unit are very diverse, and range from greenish black, light bluish gray, pale yellowish brown to pinkish gray. Unit 4 is composed of $60 \%$ clay-, $25 \%$ silt-, and $15 \%$ sand-sized material, and contains $40 \%$ nannofossils and $10-15 \%$ foraminifers. Clay minerals form $10 \%-15 \%$ of the sediment and the authigenic carbonate $10 \%$. The remaining $20 \%$ includes zeolite, opaque minerals, feldspars, and glauconite. The bedding in this unit is not readily apparent, except as color banding. Most burrows are parallel to the bedding, but some are at angles up to $90^{\circ}$ with the bedding.

Slumped material occurs in the unit at several levels. Core 19 (upper Paleocene) contains a $20-\mathrm{cm}$ thick bed of slump breccia (Sample 19-4, 130-150 cm). This breccia consists of greenish gray, angular to subrounded pebble-size chalk fragments. The age of the slumped material is early Maestrichtian to midCampanian.

Core 24 (Sections 3-6) and Core 25 (Section 1, 0-135 $\mathrm{cm}$ ) (lower Paleocene) contain three beds of slump material, from $80 \mathrm{~cm}$ to 4 meters thick. This material is middle to early Maestrichtian in age and hence is slightly younger than the slumped breccia of Core 19. Slumped material of Cores 24 and 25 is marly siliceous chalk, dark yellowish brown to greenish gray. Softsediment deformation is apparent in one of the slump beds.

A 1.5-meter-thick bed of dolomitic calcareous chalk occurs in Core 28.

\section{Unit 5}

Unit 5 extends from Core 31 to Core 38. It is composed of marly calcareous chalks which are sometimes slightly dolomitic. The unit is distinguished from the overlying unit by its higher clay-mineral content. Texturally the sediment is $65 \%$ clay-, $20 \%$ silt-, and $10 \%-15 \%$ sand-sized material, and contains $20 \%$ nannofossils, $10 \%$ foraminifers, and as much as $25 \%$ clay minerals. Quartz makes up $10 \%$ of the sediment, feldspar $5 \%$. The remaining $30 \%$ is mica, opaque minerals, and radiolarians. Color in this unit varies from light gray to dark greenish gray to pale red.

The unit is moderately indurated. Foraminifers have been recrystallized and appear as small soft white specks. The unit is well bedded; bedding is readily apparent because of bands of various shades of gray.

Inoceramus fragments are present from a depth of 50 meters in the unit to its bottom. Fragments are generally 2-5 mm wide and 2-5 cm long. A 2-cm-thick coarse layer of brown sand-sized grains composed of glauconite and iron oxides occurs in Section 35-1. Other dark bands of calcareous mud, usually about 1 $\mathrm{cm}$ thick, are also present within the unit.

A small, sub-rounded basalt pebble, about $3.5 \mathrm{~cm}$ long and $2 \mathrm{~cm}$ thick, was recovered from Section 38-3, along with pebble-sized fragments of dark mudstone. The basalt pebble is dark gray and contains phenocrysts of oxidized olivine, clinopyroxene, and 
plagioclase. Thin-section examination indicates that the basalt is highly altered. Groundmass consists of a mixture of plagioclase laths, altered $\mathrm{Fe}-\mathrm{Mg}$ silicates, probably layer-lattice silicates such as chlorite or serpentine, and titaniferous magnetite (see Fodor et al., this volume).

\section{Unit 6}

This unit extends from Core 39 to Core 41, Section 4. It is composed of clay-pebble conglomerates and mudstones. The pebbles consist of calcareous mudstone, zeolitic siltstone, ferruginous mudstone, and basalt pebbles and cobbles. Fine-grained layers are silty claystone, calcareous mudstone, and dolomitic calcareous mudstone. Color in the unit varies from light gray to dark gray. Textural composition is $60 \%$ clay-, $25 \%-30 \%$ silt-, and 10\%-15\% clay-sized material. Only traces of foraminifers and nannofossils occur, but authigenic carbonate (recrystallized calcareous biogenic material) forms $10 \%$ of the sediment. Clay minerals make up $40 \%-45 \%$ of the sediment, quartz $20 \%$, and feldspar $10 \%$. The remaining $15 \%$ includes limonite, opaque minerals, mica, glauconite, pyrite, and heavy minerals.

This unit is well indurated and well bedded. Burrowing and graded bedding are common. The conglomerates occur as several discrete layers $(30 \mathrm{~cm}$ to $2 \mathrm{~m}$ thick) in the top part of the unit, and grade upward into dark gray silty claystone. Each conglomerate-tomudstone bed is separated from the next by a light gray calcareous mudstone bed. Because of intense alteration, the composition and nature of basalt pebbles in Core 39 cannot be determined. The lower part of the unit consists of alternate bands ( $30-50 \mathrm{~cm}$ thick), dark gray, medium gray, and greenish-gray, with sharp and gradational contacts. The dark gray bands of silty mudstone contain pyrite nodules and stringers ranging from $2 \mathrm{~mm}$ to $2 \mathrm{~cm}$ in size. The higher bands consist of calcareous mudstone or dolomitic calcareous mudstone. Organic carbon content is as high as $4.8 \%$ in Core 41 (Section 2).

Inoceramus fragments are infrequent. Thin calcite layers (approximately $1 \mathrm{~cm}$ thick) occur at the base of the unit.

Unit 6 is the most distinctive unit at Site 356. The conglomerates and dark claystones make it very easy to distinguish from units above and below.

\section{Unit 7}

Unit 7 begins in Core 41 , Section 4 , and continues at least to the bottom of the hole. The sediment is well indurated and light bluish-gray to medium dark gray, with occasional white and green bands. The unit is composed primarily of marly dolomitic limestones and has a textural composition of $45 \%-50 \%$ clay-, $35 \%$ silt-, and $15 \%-20 \%$ sand-sized components. It contains $50 \%$ authigenic carbonate (recrystallized calcareous biogenic material), $15 \%$ dolomite, $15 \%$ clay minerals, and $10 \%$ quartz. The remaining $10 \%$ includes feldspar, heavy minerals, opaque minerals, glauconite, and iron oxides.

The unit is very well bedded and shows graded beds, load casts, and burrows. Contacts between beds are fairly sharp. Beds are only $10-20 \mathrm{~cm}$ thick. The lighter beds are dolomitic limestone or marly dolomitic limestone, and the darker ones are calcareous mudstone. The white laminae, recrystallized carbonate, are sometimes only $2-5 \mathrm{~cm}$ apart, and range from $1 \mathrm{~mm}$ to $1 \mathrm{~cm}$ in thickness. A few coarse layers with graded bedding also occur. They contain glauconite, quartz, iron oxides, and recrystallized limestone grains. Unit 7 is distinguished from Unit 6 by its color, banding, and composition.

\section{GEOCHEMISTRY}

We measured $p \mathrm{H}$, alkalinity, salinity, and $\mathrm{Ca}^{++}$, and $\mathrm{Mg}^{++}$, contents of twelve interstitial water samples onboard ship. Data are presented in Table 4 and Figure 9.

\section{PHYSICAL PROPERTIES}

The measured and computed physical properties at Site 356 correlate remarkably well with the lithology of the sequence. Three of six lithologic boundaries are represented by marked discontinuities superimposed on the compaction trend. A rather high degree of seismic anisotropy is also apparent. Only velocities measured in the vertical are recorded here. Corresponding horizontal values are discussed in a later chapter (Carlson and Christensen, this volume).

Measurements of acoustic velocity at Site 356 by the previously described delay line method proved an unqualified success. Standards were checked after every five or six sample measurements, often more frequently. For 51 standard measurements, the range of error was 0.04 to $0.95 \%$, (average of $0.34 \%$ ).

Physical properties data from Site 356 are summarized in Table 5 and Figure 10, according to the format described for Site 354. The uppermost unit consists of poorly consolidated oozes, characterized by low acoustic velocities which increase slowly with depth to a maximum of 1.63 to $1.64 \mathrm{~km} / \mathrm{sec}$. A shallow gradient also appears to occur in wet bulk density, though there is significant scatter in the density data. Water content shows a marked decrease within the ooze unit, beginning at about 125 meters. There is only a suggestion of increasing density in the same interval.

The silicified chalk unit (222 to $294.5 \mathrm{~m}$ ) stands out dramatically. The acoustic velocities in this unit are widely scattered and high; they reach a maximum of more than $2.5 \mathrm{~km} / \mathrm{sec}$ and the minimum is $1.75 \mathrm{~km} / \mathrm{sec}$. The average of 18 measurements for the unit is 2.07 $\mathrm{km} / \mathrm{sec}$. The highest velocities occur in the uppermost part of the chalk, as do markedly higher density values.

The lower limit of the silicified chalk is marked by a pronounced velocity inversion, which is also reflected in a slight increase in water content within the upper part of the underlying unit of nannofossil and nannofossil-foraminifer oozes. Within this unit, which extends to a depth of 432 meters, velocity, density, and water content show the strongest gradients in the section. The nannofossil-foraminifer ooze cannot be clearly distinguished from the underlying marly calcareous chalk. Though the velocities and densities in the chalk appear to be nearly constant, the previously 
TABLE 4

Summary of Shipboard Geochemical Data, Site 356

\begin{tabular}{|c|c|c|c|c|c|c|c|c|}
\hline Sample & $\begin{array}{c}\text { Sample } \\
\text { (Interval in } \mathrm{cm} \text { ) }\end{array}$ & $\begin{array}{l}\text { Subdepth } \\
\text { (m) }\end{array}$ & $p \mathrm{H}$ & $\begin{array}{l}\text { Alkalinity } \\
(\mathrm{meq} / \mathrm{l})\end{array}$ & $\begin{array}{c}\text { Salinity } \\
(\% \circ)\end{array}$ & $\underset{(\mathrm{mmoles} / 1)}{\mathrm{Ca}++}$ & $\underset{(\text { mmoles/l) }}{\mathrm{Mg++}}$ & Remarks \\
\hline 27 & $2-3,144-150$ & 14 & 7.38 & 2.54 & 34.9 & 11.22 & 53.10 & \\
\hline 28 & $4-5,144-150$ & 64.5 & 7.28 & 2.96 & 34.9 & 15.59 & 48.77 & \\
\hline 29 & $6-4,144-150$ & 120 & 7.27 & 3.36 & 34.6 & 20.50 & 44.64 & \\
\hline 30 & $10-3,144-150$ & 223 & 7.39 & 1.93 & 34.4 & 26.91 & 41.41 & \\
\hline 31 & $12-1,142-150$ & 248.5 & 7.24 & 2.36 & 34.4 & 31.23 & 36.42 & \\
\hline 32 & $17-3,142-150$ & 299 & 7.36 & 2.30 & 34.1 & 39.72 & 28.74 & \\
\hline 33 & $22-5,144-150$ & 349.5 & 7.29 & 0.60 & 34.6 & 37.58 & 31.17 & \\
\hline 34 & $27-5,144-150$ & 397.0 & 7.12 & 1.54 & 34.4 & 43.09 & 26.58 & \\
\hline 35 & $32-5,132-150$ & 464.5 & 7.80 & 0.26 & 35.2 & 47.21 & 21.48 & \\
\hline 36 & $34-2,144-150$ & 516 & 7.75 & - & 34.9 & 55.41 & 16.25 & \\
\hline 37 & $37-5,140-150$ & 606 & - & - & 36.0 & 65.34 & 11.19 & Not enough $\mathrm{H}_{2} \mathrm{O}$ for $p \mathrm{H}$, Alk \\
\hline 38 & $38-2,0-10$ & 649 & - & - & 36.0 & 66.68 & 10.66 & Not enough $\mathrm{H}_{2} \mathrm{O}$ for $p \mathrm{H}$, Alk \\
\hline
\end{tabular}
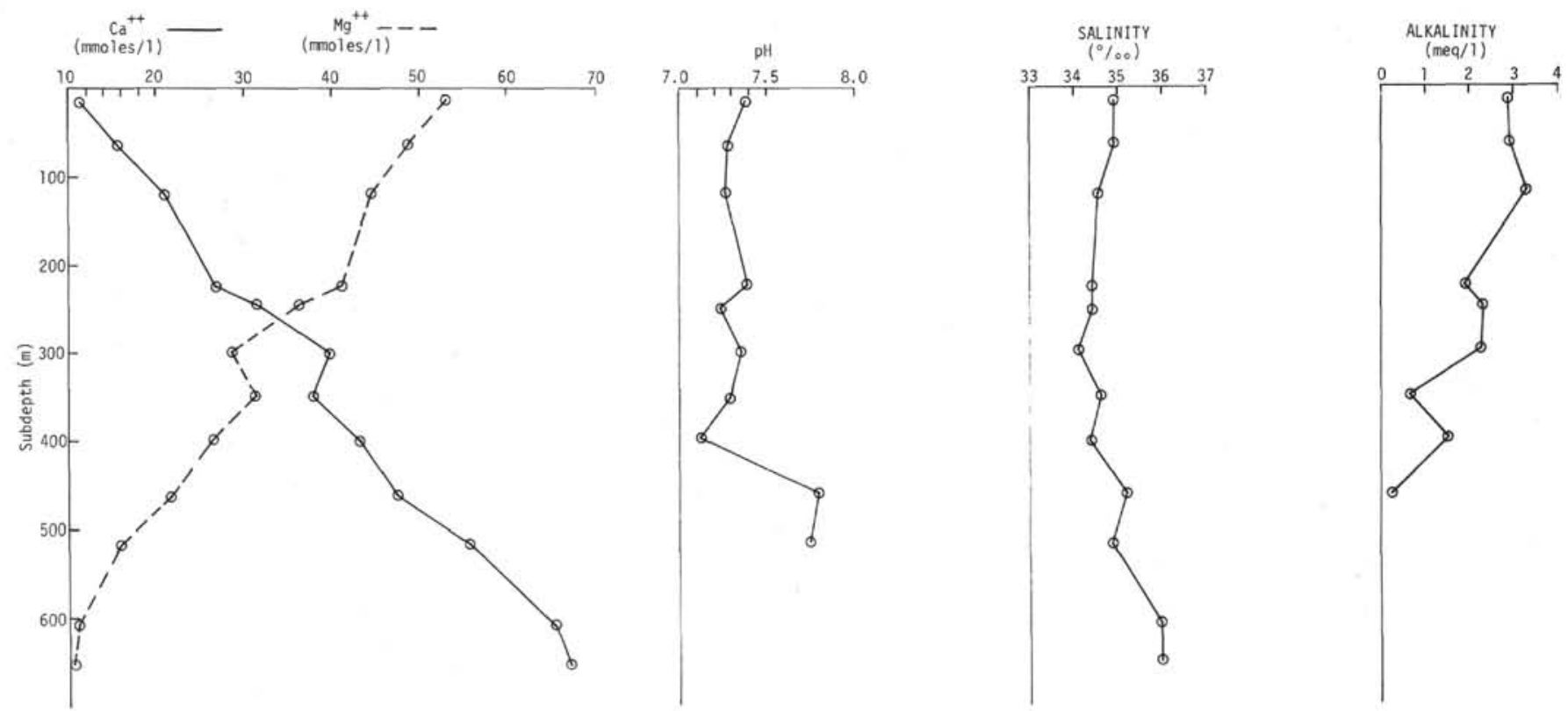

Figure 9. Geochemical parameters ( $\mathrm{pH}$, salinity, alkalinity, Ca++, and $\mathrm{Mg}++$ content) of interstitial waters versus depth, Site 356.

discussed gradient extends to a depth of about 500 meters. The velocity and density maxima at this depth are followed by a modest inversion, extending to 600 meters, which is also evident in the water content data.

The lowermost mudstone units in the section are easily distinguished from the overlying chalks by their higher maximum velocities and densities and by much greater scatter in the data.

\section{BIOSTRATIGRAPHIC SUMMARY}

An extensive sequence of Cretaceous and Tertiary deposits was cored at this site. Calcareous fossils are abundant in most samples. Radiolarians and diatoms are abundant to common only in the lower Miocene and the middle Eocene, and occur in trace quantities in the lower Eocene and the Albian. The combined stratigraphic zonations, based on planktonic foraminifers, calcareous nannofossils, silicoflagellates, and radiolarians, are presented in Figure 11.

Most of the Neogene is missing at Site 356. The top cores contain Pleistocene mixed into Pliocene. A hiatus separates these sediments from a thick lower Miocene sequence. We were able to use both calcareous and siliceous fossils to zone the lower Miocene, which is complete at this site. A second hiatus occurs between the lowermost Miocene and the middle Eocene. Below the hiatus, poorly preserved calcareous faunas and floras of middle Eocene and early Eocene age are present. A third hiatus spans the lowermost Eocene and the upper Paleocene. Below this hiatus, however, we cored a thick and nearly complete sequence of Paleocene deposits, the highlight of which is a very thick and nearly complete Danian section (Cores 25-29, about $50 \mathrm{~m}$ thick). We recognized, together with large quantities of reworked upper Maestrichtian fossils, very abundant, well preserved, and diverse Danian calcareous faunas and floras. This is one of the best preserved and thickest Danian deposits retrieved so far from the deep sea. In addition, it contains the "oldest" Danian levels so far described.

We place the Cretaceous/Tertiary boundary at Sample 29-3, $33 \mathrm{~cm}$. Below this level, no more Danian 
TABLE 5

Physical Properties Data, Site 356

\begin{tabular}{|c|c|c|c|c|c|c|c|c|c|c|c|c|}
\hline \multirow{2}{*}{$\begin{array}{c}\text { Sample } \\
\text { (Interval in } \mathrm{cm} \text { ) }\end{array}$} & \multirow{2}{*}{$\begin{array}{l}\text { Depth } \\
\text { (m) }\end{array}$} & \multirow{2}{*}{$\begin{array}{l}\text { Velocity } \\
(\mathrm{km} / \mathrm{sec})\end{array}$} & \multicolumn{3}{|c|}{ Density $(g / c c)$} & \multirow[b]{2}{*}{ Wt $\%$} & \multicolumn{3}{|c|}{ Porosity (\%) } & \multicolumn{3}{|c|}{ Acoustic Impedance } \\
\hline & & & $\mathrm{S}$ & I & G & & S & I & G & $S$ & I & G \\
\hline $3-1,45$ & 28.45 & 1.535 & - & 1.520 & 1.668 & - & - & 70.45 & 61.61 & 2.49 & 2.33 & 2.56 \\
\hline $3-1,45$ & 38.48 & - & 1.623 & - & - & 40.88 & 64.30 & - & - & - & - & - \\
\hline $3-2,91$ & 40.41 & 1.520 & 1.560 & - & - & 42.58 & 68.06 & - & - & 2.37 & - & - \\
\hline $3-3,130$ & 42.30 & 1.539 & 1.591 & - & - & 42.07 & 66.21 & - & - & 2.45 & - & - \\
\hline $3-4,45$ & 42.95 & 1.529 & 1.579 & - & - & 44.09 & 66.93 & - & - & 2.41 & - & - \\
\hline $3-5,102$ & 45.02 & 1.543 & 1.564 & - & - & 43.79 & 67.82 & - & - & 2.41 & - & - \\
\hline $3-6,103$ & 46.53 & 1.535 & 1.557 & - & - & 45.32 & 68.24 & - & - & 2.39 & - & - \\
\hline $4-1,80$ & 57.80 & 1.543 & - & - & - & - & - & - & - & - & - & - \\
\hline $4-2,136$ & 59.86 & 1.529 & - & 1.490 & 1.628 & - & - & 72.24 & 64.00 & 2.42 & 2.28 & 2.49 \\
\hline $4-2,155$ & 60.06 & - & 1.581 & - & - & 44.82 & 66.81 & - & - & - & - & - \\
\hline $4-3,67$ & 60.67 & 1.517 & 1.569 & - & - & 44.10 & 67.52 & - & - & 2.38 & - & - \\
\hline $4-4,100$ & 62.50 & 1.517 & 1.583 & - & - & 44.84 & 66.69 & - & - & 2.40 & - & - \\
\hline $4-5,125$ & 64.25 & 1.542 & 1.633 & 1.539 & 1.662 & 42.10 & 63.70 & 69.31 & 61.97 & 2.52 & 2.37 & 2.56 \\
\hline $4-6,83$ & 65.33 & 1.523 & 1.533 & - & - & 44.43 & 69.67 & - & - & 2.33 & - & - \\
\hline $5-1,110$ & 86.60 & 1.571 & 1.523 & - & - & 48.02 & 70.27 & - & - & 2.39 & - & - \\
\hline $5-3,88$ & 89.38 & 1.568 & - & 1.463 & 1.628 & 45.59 & - & 73.85 & 64.00 & - & 2.29 & 2.55 \\
\hline $5-4,70$ & 90.70 & 1.594 & - & 1.493 & 1.611 & 45.67 & - & 72.06 & 65.01 & - & 2.38 & 2.57 \\
\hline $5-5,62$ & 92.12 & 1.598 & 1.606 & - & - & 42.41 & 65.31 & - & - & 2.57 & - & - \\
\hline $5-6,65$ & 93.65 & 1.526 & 1.453 & - & - & 50.70 & 74.45 & - & - & 2.22 & - & - \\
\hline $6-2,65$ & 116.15 & 1.553 & 1.542 & - & - & 44.58 & 69.13 & - & - & 2.39 & - & - \\
\hline $6-3,139$ & 118.39 & 1.531 & 1.523 & 1.436 & 1.594 & 46.06 & 70.27 & 65.46 & 66.03 & 2.33 & 2.20 & 2.44 \\
\hline $6-4,90$ & 119.40 & 1.532 & 1.572 & 1.462 & 1.563 & 46.07 & 67.34 & 73.91 & 67.88 & 2.41 & 2.24 & 2.39 \\
\hline $6-5,72$ & 120.72 & 1.532 & 1.551 & - & - & 44.70 & 68.60 & - & - & 2.38 & - & - \\
\hline $6-6,125$ & 122.75 & 1.549 & 1.617 & - & - & 41.37 & 64.66 & - & - & 2.50 & - & - \\
\hline $7-1,121$ & 134.21 & - & - & - & - & 41.87 & - & - & - & - & - & - \\
\hline $7-1,135$ & 134.35 & 1.545 & - & - & - & - & - & - & - & - & - & - \\
\hline $7-2,120$ & 135.70 & 1.551 & - & - & - & 41.65 & - & - & - & - & - & - \\
\hline $7-3,82$ & 136.82 & 1.558 & 1.570 & 1.523 & 1.678 & 40.58 & 67.46 & 70.27 & 61.01 & 2.45 & 2.37 & 2.61 \\
\hline $7-4,90$ & 138.40 & 1.550 & 1.586 & - & - & 42.17 & 66.51 & - & - & 2.46 & - & - \\
\hline $7-5,61$ & 139.61 & 1.550 & 1.608 & - & - & 40.22 & 65.19 & - & - & 2.49 & - & - \\
\hline $7-6,90$ & 141.40 & 1.572 & 1.514 & - & - & 31.99 & 70.81 & - & - & 2.38 & - & - \\
\hline $8-1,145$ & 162.95 & 1.628 & 1.681 & 1.644 & - & 33.72 & 60.84 & 63.04 & - & 2.74 & 2.58 & - \\
\hline $8-2,145$ & 164.45 & 1.593 & 1.718 & 1.613 & 1.717 & 17.40 & 58.63 & 64.90 & 58.69 & 2.74 & 2.57 & 2.74 \\
\hline $9-1,148$ & 191.48 & 1.639 & - & 1.550 & 1.631 & 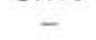 & - & 68.66 & 63.82 & - & 2.54 & 2.67 \\
\hline $9-2,48$ & 191.98 & 1.620 & - & - & 1.693 & - & - & - & 60.12 & - & - & 2.74 \\
\hline $9-2,38$ & 191.88 & 1.614 & - & - & - & - & - & - & - & - & - & - \\
\hline $10-2,148$ & 221.48 & 1.997 & - & 1.793 & 1.774 & - & - & 54.15 & 55.28 & - & 3.58 & 3.54 \\
\hline $10-3,2$ & 221.52 & 2.136 & - & 1.819 & 1.845 & - & - & 52.60 & 51.04 & - & 3.89 & 3.94 \\
\hline $10-4,15$ & 223.15 & 2.466 & - & - & - & 22.30 & - & - & - & - & - & - \\
\hline $11-1,44$ & 237.94 & 2.240 & - & 1.815 & 1.826 & 20.66 & - & 52.84 & 52.18 & - & 4.07 & 4.09 \\
\hline $11-1,52$ & 238.02 & 1.846 & - & - & - & 28.56 & - & - & - & - & - & - \\
\hline $11-2,73$ & 239.73 & 2.536 & - & 2.106 & 1.848 & 28.66 & - & 35.46 & 50.87 & - & 5.34 & 4.69 \\
\hline $11-3,98$ & 241.48 & 2.095 & - & 1.967 & 1.821 & 27.50 & - & 43.76 & 52.48 & - & 4.12 & 3.81 \\
\hline $12-1,3$ & 247.03 & 1.811 & - & 2.067 & 1.777 & 27.50 & - & 37.79 & 55.10 & - & 3.74 & 3.22 \\
\hline $12-2,103$ & 249.53 & 1.746 & - & 1.847 & 1.726 & 32.45 & - & 50.93 & 58.15 & - & 3.22 & 3.01 \\
\hline $12-2,3$ & 249.03 & 1.909 & - & - & - & - & - & - & - & - & - & - \\
\hline $13-1,132$ & 257.83 & 1.863 & - & 1.637 & - & 31.55 & - & 63.46 & - & - & 3.05 & - \\
\hline $13-2,38$ & 258.38 & 1.846 & - & 1.650 & 1.737 & 31.84 & - & 62.69 & 57.49 & - & 3.05 & 3.21 \\
\hline $14-1,18$ & 266.18 & 1.900 & - & 1.691 & 1.766 & - & - & 60.24 & 55.76 & - & 3.21 & 3.36 \\
\hline $15-1,75$ & 276.25 & 2.346 & - & - & 1.812 & - & - & - & 53.01 & - & - & 4.25 \\
\hline $15-2,125$ & 278.25 & 2.416 & - & 1.714 & 1.754 & 26.06 & - & 58.87 & 56.48 & - & 4.14 & 4.24 \\
\hline $15-3,96$ & 279.46 & 2.272 & - & 1.695 & 1.756 & - & - & 60.00 & 56.36 & - & 3.85 & 3.99 \\
\hline $16-1,88$ & 285.88 & 1.972 & - & - & - & 27.54 & - & - & - & - & - & - \\
\hline $16-2,127$ & 287.77 & 1.912 & - & 1.758 & 1.823 & 27.98 & - & 56.24 & 52.36 & - & 3.36 & 3.49 \\
\hline $17-1,23$ & 294.73 & 1.722 & - & 1.785 & 1.847 & 29.65 & - & 54.63 & 50.93 & - & 3.07 & 3.18 \\
\hline $17-2,130$ & 297.30 & 1.765 & - & 1.782 & 1.824 & 31.48 & - & 54.81 & 52.30 & - & 3.15 & 3.22 \\
\hline $17-4,137$ & 300.37 & 1.717 & - & 1.784 & 1.839 & 29.25 & - & 54.69 & 51.40 & - & 3.06 & 3.16 \\
\hline $18-1,143$ & 305.43 & 1.698 & - & 1.785 & 1.896 & 30.37 & - & 54.63 & 48.00 & - & 3.03 & 3.22 \\
\hline $18-2,111$ & 306.61 & 1.746 & - & 1.799 & 1.858 & - & - & 53.79 & 50.27 & - & 3.14 & 3.24 \\
\hline $18-3,55$ & 307.55 & - & - & 1.789 & 1.866 & - & - & 54.39 & 49.79 & - & - & - \\
\hline $18-4,95$ & 309.45 & 1.675 & - & - & - & - & - & - & - & - & - & - \\
\hline $18-6,52$ & 312.02 & 1.712 & - & 1.801 & 1.818 & 31.49 & - & 53.67 & 52.66 & - & 3.08 & 3.11 \\
\hline $19-1,142$ & 314.92 & 1.698 & - & 1.783 & 1.882 & 30.65 & - & 54.75 & 48.84 & - & 3.03 & 3.20 \\
\hline $19-2,142$ & 316.42 & 1.701 & - & 1.795 & 1.865 & 28.70 & - & 54.03 & 49.85 & - & 3.05 & 3.17 \\
\hline $19-3,103$ & 317.53 & 1.722 & - & 1.796 & 1.863 & 30.09 & - & 53.97 & 49.97 & - & 3.09 & 3.21 \\
\hline $19-4,41$ & 318.41 & 1.737 & - & - & - & - & - & - & - & - & - & - \\
\hline $19-5,148$ & 320.98 & 1.719 & - & 1.843 & 1.910 & 27.06 & - & 51.16 & 47.16 & - & 3.17 & 3.28 \\
\hline $19-6,96$ & 321.96 & 1.715 & - & - & - & - & - & - & - & - & - & - \\
\hline
\end{tabular}


TABLE 5 - Continued

\begin{tabular}{|c|c|c|c|c|c|c|c|c|c|c|c|c|}
\hline \multirow{2}{*}{$\begin{array}{c}\text { Sample } \\
\text { (Interval in } \mathrm{cm} \text { ) }\end{array}$} & \multirow{2}{*}{$\begin{array}{l}\text { Depth } \\
(\mathrm{m})\end{array}$} & \multirow{2}{*}{$\begin{array}{l}\text { Velocity } \\
(\mathrm{km} / \mathrm{sec})\end{array}$} & \multicolumn{3}{|c|}{ Density $(\mathrm{g} / \mathrm{cc})$} & \multirow[b]{2}{*}{ Wt $\%$} & \multicolumn{3}{|c|}{ Porosity (\%) } & \multicolumn{3}{|c|}{ Acoustic Impedance } \\
\hline & & & $\mathrm{S}$ & I & G & & $\mathrm{S}$ & I & G & $\mathrm{S}$ & I & G \\
\hline $20-2,96$ & 325.46 & 1.700 & - & 1.809 & 1.840 & 27.96 & - & 53.19 & 51.34 & - & 3.08 & 3.13 \\
\hline $21-1,108$ & 333.58 & 1.728 & - & 1.801 & 1.834 & 28.40 & - & 53.67 & 51.70 & - & 3.11 & 3.17 \\
\hline $22-1,127$ & 343.27 & 1.705 & - & 1.809 & 1.883 & 28.16 & - & 53.19 & 48.78 & - & 3.08 & 3.21 \\
\hline $22-2,7$ & 343.57 & 1.719 & - & - & - & - & - & - & - & - & - & - \\
\hline $22-3,51$ & 345.51 & 1.706 & - & - & 1.879 & 27.62 & - & - & 49.01 & - & - & 3.21 \\
\hline $23-1,47$ & 351.97 & 1.728 & - & 1.828 & 1.906 & 27.29 & - & 52.06 & 47.40 & - & 3.16 & 3.29 \\
\hline $23-3,118$ & 354.98 & 1.774 & - & - & - & 25.49 & - & - & - & - & - & - \\
\hline $23-2,145$ & 354.45 & 1.784 & - & - & - & - & - & - & - & - & - & - \\
\hline $23-4,144$ & 357.44 & 1.740 & - & 1.876 & 1.930 & 24.27 & - & 49.19 & 45.97 & - & 3.26 & 3.36 \\
\hline $24-1,149$ & 362.49 & 1.783 & - & 1.902 & 1.955 & 23.20 & - & 47.64 & 44.48 & - & 3.39 & 3.49 \\
\hline $24-2,148$ & 363.98 & 1.817 & - & - & - & - & - & - & - & - & - & - \\
\hline $24-3,149$ & 365.49 & 1.835 & - & - & 1.900 & 24.76 & - & - & 47.76 & - & - & 3.49 \\
\hline $24-5,149$ & 368.49 & 1.883 & - & 1.871 & 1.961 & 22.83 & - & 49.49 & 44.12 & - & 3.52 & 3.69 \\
\hline $25-1,147$ & 371.97 & 1.871 & - & - & - & - & - & - & - & - & - & - \\
\hline $25-2,103$ & 373.03 & 1.703 & - & 1.871 & 2.099 & 21.82 & - & 49.49 & 35.88 & - & 3.19 & 3.57 \\
\hline $25-3,107$ & 374.57 & 1.845 & - & - & - & - & - & - & - & - & - & - \\
\hline $25-4,108$ & 376.08 & 1.839 & - & - & 1.717 & 23.74 & - & - & 58.69 & - & - & 3.16 \\
\hline $26-1,58$ & 380.58 & 1.936 & - & 1.956 & 2.029 & 22.63 & - & 44.42 & 40.06 & - & 3.79 & 3.93 \\
\hline $26-3,12$ & 383.12 & 1.804 & - & 1.893 & 1.919 & 24.27 & - & 48.18 & 44.63 & - & 3.41 & 3.46 \\
\hline $26-6,36$ & 387.86 & 1.785 & - & - & 1.961 & - & - & - & 44.12 & - & - & 3.50 \\
\hline $27-1,4$ & 389.54 & 1.858 & - & 1.905 & 2.003 & 22.55 & - & 47.46 & 41.61 & - & 3.54 & 3.72 \\
\hline $27-5,45$ & 395.95 & 1.925 & - & - & - & - & - & - & - & - & - & - \\
\hline $28-1,133$ & 400.33 & 1.813 & - & 2.080 & 2.008 & 22.92 & - & 37.01 & 41.31 & - & 3.77 & 3.64 \\
\hline $28-3,87$ & 402.87 & 1.866 & - & 1.921 & 1.985 & 23.44 & - & 46.51 & 42.69 & - & 3.58 & 3.70 \\
\hline $28-6,107$ & 407.57 & 1.995 & - & - & - & - & - & - & - & - & - & - \\
\hline $29-2,148$ & 411.48 & 1.798 & - & 1.808 & 1.890 & 27.45 & - & 53.25 & 48.36 & - & 3.25 & 3.40 \\
\hline $29-3,148$ & 412.98 & 1.944 & - & 2.051 & 2.111 & 18.16 & - & 38.75 & 35.16 & - & 3.99 & 4.10 \\
\hline $29-4,63$ & 413.63 & 1.950 & - & - & - & - & - & - & - & - & - & - \\
\hline $29-6,107$ & 417.07 & 1.939 & - & - & - & - & - & - & - & - & - & - \\
\hline $30-6,3$ & 425.53 & 1.861 & - & 1.989 & 2.130 & 19.01 & - & 42.45 & 34.03 & - & 3.70 & 3.96 \\
\hline $31-2,24$ & 438.74 & 1.910 & - & - & - & - & - & - & - & - & - & - \\
\hline $31-6,15$ & 444.65 & 1.886 & - & 2.062 & 2.135 & 19.59 & - & 38.09 & 33.73 & - & 3.89 & 4.03 \\
\hline $32-1,117$ & 457.17 & 1.968 & - & 2.125 & 2.168 & 16.01 & - & 34.33 & 31.76 & - & 4.18 & 4.27 \\
\hline $32-3,30$ & 459.30 & 1.968 & - & - & - & - & - & - & - & - & - & - \\
\hline $33-2,132$ & 487.32 & 2.036 & - & 2.163 & 2.237 & 15.18 & - & 32.06 & 27.64 & - & 4.40 & 4.55 \\
\hline $34-1,148$ & 514.48 & 2.149 & - & 2.195 & 2.260 & 13.94 & - & 30.15 & 26.27 & - & 4.72 & 4.86 \\
\hline $34-3,148$ & 517.48 & 2.099 & - & 2.153 & - & 14.32 & - & 32.66 & - & - & 4.52 & - \\
\hline $34-6,134$ & 521.84 & 2.070 & - & - & - & - & - & - & - & - & - & - \\
\hline $35-1,140$ & 542.90 & 2.104 & - & 2.172 & 2.210 & 14.09 & - & 31.52 & 29.25 & - & 4.57 & 4.65 \\
\hline $35-3,29$ & 544.79 & - & - & - & - & - & - & - & - & - & - & - \\
\hline $35-6,73$ & 549.73 & 2.035 & - & 2.094 & 2.158 & 16.26 & - & 36.18 & 32.36 & - & 4.26 & 4.39 \\
\hline $36-1,18$ & 570.18 & 2.073 & - & 2.132 & 2.174 & 17.01 & - & 33.91 & 31.40 & - & 4.42 & 4.51 \\
\hline $36-4,40$ & 574.90 & 2.077 & - & - & - & - & - & - & - & - & - & - \\
\hline $37-2,146$ & 601.46 & 2.242 & - & 2.138 & 1.921 & - & - & 33.55 & 46.51 & - & 4.79 & 4.31 \\
\hline $37-3,149$ & 602.99 & 2.345 & - & - & - & - & - & - & - & - & - & - \\
\hline $37-5,58$ & 605.08 & 2.286 & - & - & - & - & - & - & - & - & - & - \\
\hline $37-0,0$ & 608.00 & 2.340 & - & 2.194 & 2.044 & - & - & 30.21 & 39.16 & - & 5.13 & 4.78 \\
\hline $38-1,149$ & 647.49 & 2.289 & - & 2.189 & 2.044 & 14.24 & - & 30.51 & 39.16 & - & 5.01 & 4.68 \\
\hline $38-3,8$ & 649.08 & 2.340 & - & - & - & - & - & - & - & - & - & - \\
\hline $39-2,149$ & 677.49 & 3.372 & - & 2.201 & 2.034 & 13.61 & - & 29.79 & 39.76 & - & 7.42 & 6.86 \\
\hline $39-4,142$ & 680.42 & 2.288 & - & 2.184 & 2.021 & 13.83 & - & 30.81 & 40.54 & - & 5.00 & 4.62 \\
\hline $39-6,88$ & 682.88 & 2.592 & - & - & - & - & - & - & - & - & - & - \\
\hline $40-1,65$ & 694.15 & 2.463 & - & 2.224 & 2.015 & 13.83 & - & 28.42 & 40.90 & - & 5.48 & 4.96 \\
\hline $40-3,127$ & 697.77 & 2.292 & - & - & 1.949 & - & - & - & 44.84 & - & - & 4.47 \\
\hline $40-6,84$ & 701.84 & - & 2.263 & 2.050 & - & - & 12.06 & 38.81 & - & 5.19 & 4.70 & - \\
\hline $40-6,62$ & 701.62 & 2.294 & - & - & - & 12.06 & - & - & - & - & - & - \\
\hline $41-2,48$ & 704.98 & 2.221 & - & 20.96 & 1.932 & 17.62 & - & 36.06 & 45.85 & - & 4.66 & 4.29 \\
\hline $41-4,53$ & 708.03 & 2.056 & - & - & 1.832 & - & - & - & 51.82 & - & - & 3.77 \\
\hline $41-4,119$ & 708.69 & 2.845 & - & 2.406 & 2.198 & 9.40 & - & 17.55 & 29.97 & - & 6.85 & 6.25 \\
\hline $41-5,125$ & 710.25 & 2.839 & - & - & 2.167 & - & - & - & 31.82 & - & - & 6.15 \\
\hline $42-1,105$ & 713.55 & 2.861 & - & 2.343 & 2.209 & 8.08 & - & 21.31 & 29.31 & - & 6.70 & 6.32 \\
\hline $42-3,144$ & 716.94 & 2.404 & - & - & - & - & - & - & - & - & - & - \\
\hline $42-6,147$ & 721.47 & 3.952 & - & 2.896 & 2.239 & - & - & - & 27.52 & - & 8.44 & 8.85 \\
\hline $43-1,148$ & 723.48 & 2.865 & - & 2.261 & 2.081 & 11.13 & - & 26.21 & 36.96 & - & 6.48 & 5.96 \\
\hline $43-3,2$ & 725.02 & 2.799 & - & - & 2.087 & - & - & - & 36.60 & - & - & 5.84 \\
\hline $43-5,148$ & 729.48 & 3.524 & - & 2.353 & 2.201 & 8.82 & - & 20.72 & 29.79 & - & 8.29 & 7.76 \\
\hline $44-1,146$ & 732.96 & - & - & - & 2.189 & - & - & - & 30.51 & - & - & - \\
\hline $44-3,108$ & 735.58 & 2.658 & - & - & 2.047 & - & - & - & 38.99 & - & - & 5.44 \\
\hline
\end{tabular}

NOTE: $\mathrm{S}=$ syringe technique, $\mathrm{I}=$ immersion technique, $\mathrm{G}=$ GRAPE. 


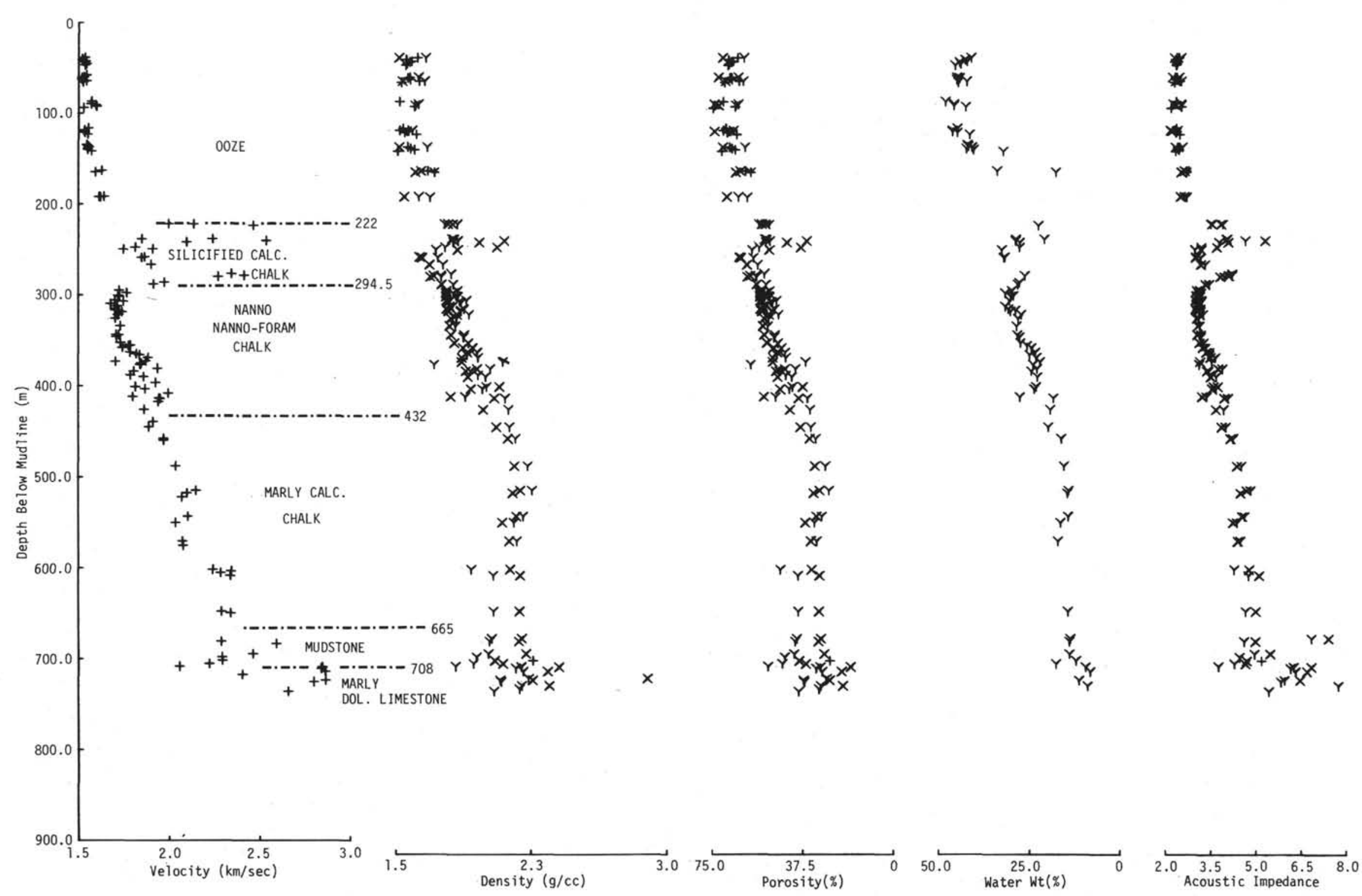

Figure 10. Physical properties versus depth;,$+ x$, and $y$, represent syringe, immersion, and GRAPE values, respectively. 


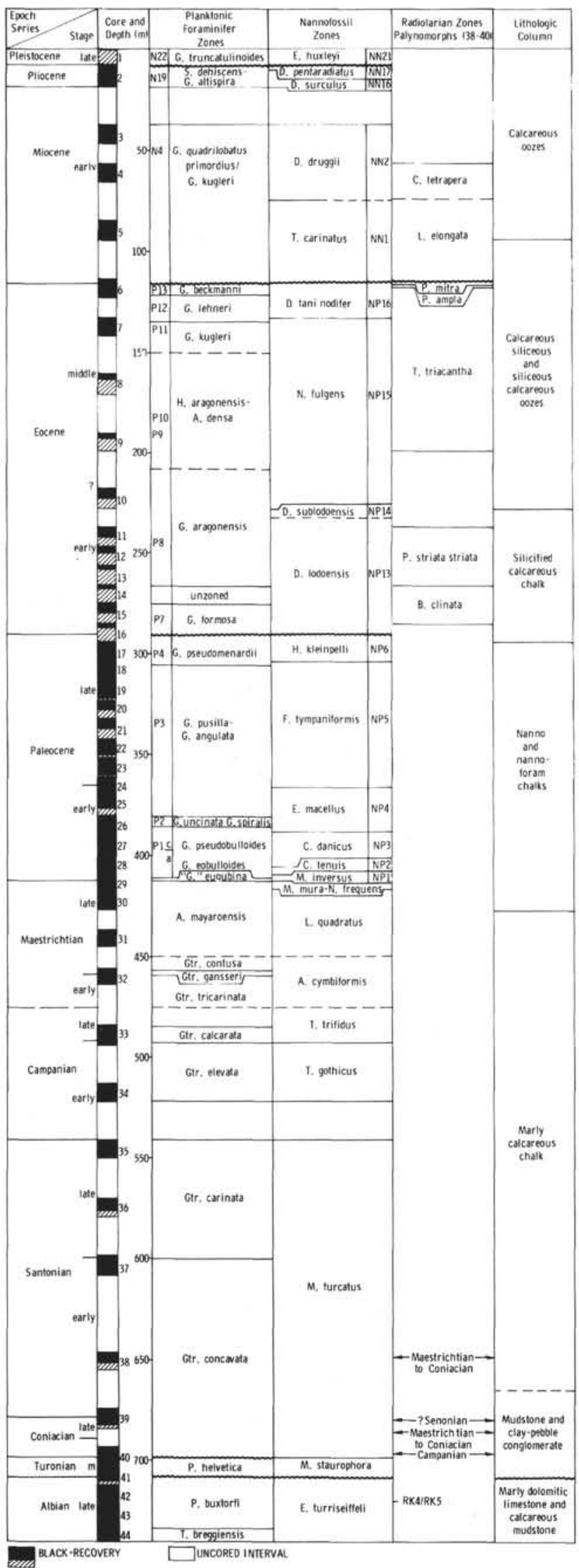

Figure 11. Biostratigraphic summary, Site 356. fossils occur and the lithology changes markedly. Diverse upper Maestrichtian to Coniacian planktonic faunas and floras are present. Although we identified the complete sequence of zones, small sections of zones are apparently missing. Reworking is also common, particularly in the Campanian sediments. Dissolution has destroyed the foraminifer faunas of parts of the lower and upper Campanian section at this site.

The foraminifers in Core 41 indicate a middle Turonian age, whereas the calcareous nannofossils indicate a late Turonian age. The deposits between Core 39 and Section 41-4 represent part of an episode of sapropel formation at this site from the middle to late Turonian through the early Santonian. The sapropels are separated from the underlying indurated limestone by a sharp lithologic break. The entire Cenomanian and lower Turonian are apparently missing, since the limestone yielded a fauna and flora of late Albian age. This upper Albian limestone, which continues to the bottom of the hole, contains plentiful calcareous fossils (some of them presumably redeposited from a topographic high and/or by turbidites), as well as the only common radiolarians of Cretaceous age.

\section{Paleoecology}

Site 356 lies in the present climatic "temperate" zone and the latitudinal "subtropics." Sediment there, being thus situated, is apt to contain varying percentages of the so-called tropical and subtropical species. The presence and/or relative percentages of these climatic indicator species (or faunas) in the past can provide information regarding the paleoclimatology of this area, as well as the paleochemistry of water masses in this area and paleocirculation patterns.

Depth-restricted foraminifers, primarily the benthic species, and ostracodes and some species of coccoliths can further provide information on the depositional depths of this site and can signal the presence of reworked material.

\section{Water Depth and Redeposition of Fossils}

Throughout this sequence the benthic foraminifers indicate deposition of sediment at depths between about 1000 and 3000 meters. Depths closer to the deeper limit are indicated at the top of the sequence (Tertiary) and depths toward the shallower limit in the Cretaceous part of the sequence. The ostracodes, dating back to about 75 m.y.B.P., do not indicate any time wherein shallow faunas were present (Benson, this volume).

Calcareous nannofossils-the presence of $Z$. bijugatus and high diversity of nannofossil faunas - suggest a possible shallower origin, less than 1000 meters, for Eocene sediments. In the sediments of Late Cretaceous (late Campanian and Maestrichtian) age, the presence of $K$. magnificus indicates shallower conditions. The presence of littoral diatoms in the middle Eocene sediment supports the evidence of the calcareous nannofossils for relatively shallow accumulation conditions or transport from littoral areas into deeper depths (Fenner, this volume). 
The in-situ benthic foraminifers corroborate a shallower water depth in the Cretaceous, but not in the early Tertiary. Since slumping and redeposition have mixed faunas from different depths, it may not be possible to resolve the inconsistencies between the foraminifers and the nannofossil and diatom data.

\section{Paleotemperatures and Paleocirculation}

\section{Tertiary}

Both tropical and subtropical components are present in most of the Tertiary faunas. However, evidence from the mid-Eocene foraminifers, coccoliths, and radiolarians indicates a significant change in water temperature and/or circulation.

The low discoaster/chiasmolithus ratio in both middle and lower Eocene faunas indicates cooler water (although see Perch-Nielsen, this volume). An incursion of cooler water species into the middle and lower Eocene radiolarian faunas confirms this indication.

A change below Sample 6, CC in the ratio of keeled globorotalids and acute-edged acaraninids to globigerinids and round-edged acaraninids indicates cooler waters in the mid-Eocene. All keeled globorotalids, except for an occasional $G$. aragonensis, disappear in the lower Eocene faunas at this site.

Studies of the biogeographic patterns of planktonic foraminifers in the subtropics depict similar faunal changes (Premoli-Silva and $\mathrm{Haq}$, in preparation). Keeled globorotalids, except for G. aragonensis, disappeared in the early Eocene after the time of $G$. formosa at middle latitudes. This disappearance has been correlated with the first buildup of Antarctic land ice (Kennett, 1974, unpublished) and with the contemporaneous cooling of deep waters in the Southern Hemisphere (Shackleton and Kennett, 1974).

The middle Eocene was a time of generally carbonate-poor facies in the Atlantic (Premoli-Silva and Haq, in preparation), as well as of increased latitudinal differentiation of planktonic foraminifer and nannofossil assemblages.

The two main hiatuses in the Tertiary cover the time from late middle Eocene through Oligocene and late early Miocene through Pliocene. It may be no coincidence that these were also the coldest periods of the Tertiary, as indicated by high-latitude paleotemperature curves by Shackleton and Kennett (1974) and Boersma and Shackleton (this volume). A warming trend, i.e., warm relative to the Oligocene and later Miocene, characterized the early Miocene, and may be responsible for the presence of sediments of that age at this site.

There is one significant piece of evidence from the nannofossils which indicates restricted circulation or special conditions in this area in the earliest Danian. In Core 29-1, a Thoracosphaera-Braarudosphaera chalk occurs. Haq et al. (this volume) find that similar chalks are an oceanwide phenomenon in the lowermost Danian indicating oceanwide special conditions during that time, and because it indicates restricted environments in other parts of the world, it may have similar significance here. It is here taken as a local rather than oceanwide phenomenon.

Cretaceous

There is generally a stratigraphic hiatus at the Cretaceous/Tertiary boundary, reflecting the events which resulted in mass extinctions of faunas and floras at the end of the Maestrichtian. The completeness of the section at this site is contrary to the suggestion of Worsley (1974) that good Maestrichtian/Paleocene calcareous sections should be found only on the shelf, since the CCD in the open ocean had shallowed into the photic zone, precluding abundant deposition of carbonate at any great depth. Site 356 , however, lay close to 1000 meters in the Late Cretaceous.

\section{Foraminifers}

Cores taken at this site on the São Paulo Plateau contain foraminifers ranging in age from Pleistocene to Early Cretaceous. At least one sample per section from all 44 cores was examined for foraminifers.

\section{Plio/Pleistocene}

Although only the core catcher from Core 1 contains slight amounts of sediment, a diverse tropical assemblage of planktonic foraminifers occurs; it includes Globorotalia truncatulinoides, $G$. inflata, $G$. cultrata, G. tumida, G. flexuosa, Pulleniatina obliquiloculata, and pink Globigerinoides ruber. This assemblage is well preserved, and many forms still have visible spines and spine bases. Benthic foraminifers are rare, but the genera Lagena, Pyrgo, Laticarinina, and hispid and hispido-costate Uvigerina can be recognized. Smooth and spiny ostracodes are also present.

The fauna is of Pleistocene age. The pink G. ruber indicate the late Pleistocene and $G$. flexuosa, which became extinct at $80,000 \mathrm{yr}$ B.P., sets an upper age limit. The benthic foraminifers are bathyal.

Section 2-1 through Sample 2, CC contains Pleistocene fossils mixed in with the Pliocene species Globorotalia miocenica, G. multicamerata, Globigerinoides obliquus, and Neogloboquadrina humerosa. There is apparently extensive mixing throughout this core, but the age is considered to be Pliocene (N19), older than the extinction of Sphaeroidinellopsis, but younger than the extinction of Globorotalia margaritae.

\section{Miocene}

Cores 3-5 all contain sediments of early Miocene age.

The faunas in all three cores, very similar in their content, include Globorotalia (Turborotalia) kugleri, Globoquadrina dehiscens, G. altispira, Catapsydrax sp., and in Core 5, Globigerinoides primordius. There are few benthic forms in these samples; those present belong to the genera Cassidulina, Stilostomella, and Laticarinina. Occasional fish teeth and echinoid fragments are present, and radiolarians and diatoms are common in Cores 4 and 5 .

These faunas all appear to belong in the lowermost Miocene (N4-N5). The lower portion of Core 5, however, has an "Oligocene character." Recognition of passage from the Miocene into the uppermost 
Oligocene is still difficult because definitive criteria are lacking.

\section{Eocene}

Eocene sediments were recovered in Cores 6-15. The sediments of middle Eocene age (Cores 6-10) vary from chalky, foraminifer-rich sediments to radiolarian and diatom-rich sediments which contain only a very small proportion of foraminifers. Those sediments composed predominantly of radiolarians characteristically contain more diverse assemblages of planktonic foraminifers and a greater percentage (relative to globigerinids) of keeled globorotalids. In the radiolarian-rich samples, however, benthic foraminifers are scarcer, if present at all.

Samples from Core 6 are rich in foraminifers, particularly Truncorotaloides rohri, Globorotalia (Morozovella) lehneri, G. (M.) spinulosa, Acarinina densa, A. bullbroki, A. mckannai, Pseudohastigerina micra, and Hantkenina aragonensis. Keeled globorotalids and acute-edged acaraninids are abundant; round-edged acaraninids and globigerinids are secondary in importance. Chilogumbelina and Pseudohastigerina, however, are also important components of the fauna. The preservation of this assemblage is good, and excellent in Section 6-2. Benthic foraminifers are very scarce.

Core 6 probably encompasses both the Orbulinoides beckmanni (P13) and Globorotalia lehneri (P12) zones.

Cores 7 through 10 contain less diverse faunas in which the globigerinids and round-edged acaraninids predominate. Faunas typically include "Globigerinita" howei, Globigerina (Subbotina) trilocularis, Acarinina mckannai, A. wilcoxensis, Chilogumbelina wilcoxensis, and primitive Globigerinatheka. The keeled Globorotalia (Morozovella) aragonensis first appears in Core 9.

The benthic species in all these cores are rare, but Globocassidulina, Stilostomella $(=$ Siphonodosaria), and several lagenids occasionally occur, along with a few smooth ostracodes.

The faunas are often contaminated and sometimes lacking in zonal index fossils. However, this group of cores is called middle Eocene: Cores 6, P13-P12; Core 7, P11; Core 8, P10; Core 9, P9; and Core 10, P 8.

The planktonic foraminifers between Core 6 , at the top, and Cores 7-10 also indicate a significant environmental change. Cooling is signaled by the decreased abundance of keeled globorotalids and acute-edged acaraninids below Core 6 .

Lower Eocene faunas at this site are even more depleted than those of the middle Eocene. Cores 11 through 16, when a fauna is preserved and recognizable, contain poorly preserved specimens of Acarinina wilcoxensis, A. mckannai, Globorotalia (J.) broedermanni, Globigerina (S). trilocularis, and Globorotalia $(M$.) aragonensis. Benthic foraminifers are rare.

Since many of the age-diagnostic species are absent, Cores $11-14$ are tentatively assigned to the lower Eocene, P8, and Cores 15 and 16 to P7, on the basis of right-coiled G. (M.) aragonensis (Premoli-Silva, in preparation).

\section{Paleocene}

A striking Paleocene sequence, including an excellently preserved and complete Danian section, occurs from Sample 16, CC through Sample 29-3, 33 $\mathrm{cm}$. This section, further, includes some of the oldest Tertiary samples from the "Globigerina" eugubina Zone yet recorded.

Zones P4 through P2 can be recognized from Core 17 through Section 26-3. The age-diagnostic species Globorotalia (Planorotalites) pseudomenardii, G. (P.) pusilla, $G$. (M.) angulata, and $G$. (M.) uncinata are all present in this series of cores. Many of these faunas are poorly preserved and contain admixed Cretaceous fossils.

The Danian (P1) occurs in Sections 26-5 through 293. Only P1b appears to be absent. The "Globigerina" eugubina Zone, however, is well represented in several samples, and the oldest part of this zone so far recorded is found in Core 29 at the lithologic break marking the Cretaceous/Tertiary boundary. The $G$. eugubina fauna contains, besides $G$. eugubina and $G$. fringa, the probable precursor of Globoconusa daubjergensis. Within the Danian there are also admixed very well preserved planktonic foraminifers of latest Cretaceous age. Preservation of most of this Danian sequence is remarkably good.

Benthic foraminifers are common in the Paleocene samples. Most species are bathyal, although there is a large component of admixed Cretaceous benthic species from slightly shallower depths.

\section{Cretaceous}

An extensive and fairly well preserved sequence of Cretaceous deposits, including fossils of Maestrichtian through Coniacian, Turonian, and late Albian ages, was recovered in Cores 29 through 44 . The biostratigraphic zonation of Premoli-Silva and Bolli (1973) was used to zone this sequence.

The presence in Cores 29 through 31 of Abathomphalus mayaroensis, along with Globotruncana contusa, G. ventricosa, Rugoglobigerina rugosa, and Racemiguembelina fructicosa places these cores in the upper Maestrichtian A. mayaroensis Zone. Preservation is poorer in the top two cores, but the faunas are more diverse and better preserved in Core 31 . The biserial planktonic species also become more numerous and more diverse in Core 31.

Benthic species appear mixed, apparently because of downslope redeposition. Important forms include Hyperamina and the nodosarids.

The top section of Core 32 belongs to the Globotruncana contusa Zone, but lower sections contain assemblages dominated by $G$. tricarinata, $G$. falsostuarti, G. gagnebini, G. havenensis, G. plummerae, and abundant rugotruncanids. Some anomalous benthic forms from this core are discussed below. The Maestrichtian faunas appear tropical and open marine, and seem to have been deposited at bathyal depths. The lower sections of Core 32 are assigned to the $G$. tricarinata Zone, of early Maestrichtian age.

Cores 33 and 34 contain unusual benthic faunas which include many large agglutinated forms (see 
Sliter, this volume). Among the planktonic species are Globotruncana calcarata, G. elevata, G. leupoldi, Heterohelix pulchra, and Pseudotextularia elegans. In Core 34, the first specimens of Rugoglobigerina rugosa appear. The preservation of these fossils is fair; many are broken or are recrystallizing.

The large agglutinated forms are considered redeposited; the supposed in-situ benthonic forms include a Gavelinella-Gyroidinoides fauna with Pullenia, Osangularia, Aragonia, and fewer nodosarids than in younger samples.

Cores 33 and 34 apparently span the Campanian $G$. calcarata and $G$. elevata zones.

Cores 35 through 39 and the top of 40 contain similar faunas, including $G$. fornicata, $G$. carinata, $G$. coronata, $G$. linneianna, and in the lower cores, $G$. concavata. Among the smaller fauna, Clavihedbergella simplex, Heterohelix reussi, Rugoglobigerina pilula, and Shackoina spp. are present.

The benthic faunas in this sequence of cores have an austral character, and there has been much redeposition of material, presumably down the slope. The Gyroidinoides-Gavelinella fauna predominates, along with Tritaxia, Pleurostomella, Ramulina, and Hyperamina. The faunas are thought to characterize the bathyal zone.

The faunas span the Santonian and the top of the Coniacian; both the $G$. carinata and the $G$. concavata zones are well represented.

An unusual sequence of lithologies occurs in Cores 39 through 41 (Section 4). The sediments were apparently deposited in conditions ranging from totally reducing to totally open marine.

Foraminifers occur at various, though not all, levels, and the faunas vary from planktonic ooze to a highly dissolved and depauperate benthic fauna to an Ammodiscus and a few fragments. The relationship between the types of faunas and the chemical and other properties of the sediments is being investigated. At one level in Core 40, however, an ooze intercalated into the reducing layers contains a recognizable Turonian fauna that includes the zonal marker Globotruncana helvetica.

Core 41 is replete with black layers rich in pyrite, and these layers yielded no foraminiferal residue. Section 41-4, however, contains light gray limestone, at the bottom, which is rich in planktonic foraminifers. Although the limestone is badly preserved and highly altered by diagenesis, it is possible to distinguish in it Planomalina buxtorfi, Hedbergella amabilis, $H$. delrioensis, and Heterohelix globulosa. No rotaliporids are present. This same assemblage persists from Core 41 through Section 44-4; all of these cores have been assigned to the upper Albian P. buxtorfi Zone. The lack of rotaliporids, rather than a depth of dissolution indicator, signals the austral character of this fauna. The cores also contain abundant evidence of turbidites, but the supposed in-situ benthic fauna still contains Gavelinella, deep pleurostomellids, and other bathyal taxa, suggesting a water depth close to 1000 meters.

The bottom of Core 44 yielded a foraminiferal fauna dominated by Hedbergella and small Heterohelicidae. Ticinella is rare and limited to a few "primitive" species. The presence of $T$. breggiensis allows us to recognize the $T$. breggiensis Zone at the bottom of this hole.

\section{Calcareous Nannofossils}

All 44 cores recovered at this site contain calcareous nannofossils. With a few exceptions, preservation of the nannofossils is moderate to good in the Cenozoic, deteriorates in the Upper Cretaceous samples, and becomes poor in the upper Albian limestones in which the hole bottomed.

The distribution of coccolith zones is summarized in Figure 11, and the distribution of species is shown in a chapter by Perch-Nielsen (this volume).

\section{Pleistocene}

The only sample from Core 1, Sample 1, CC, contains a subtropical assemblage of the E. huxleyi Zone and sparse reworked Pliocene forms.

\section{Pliocene}

Subtropical assemblages of the Discoaster pentaradiatus and $D$. surculus zones occur in Core 2 . The presence of scarce to a few Reticulofenestra pseudoumbilica in all samples of Core 2 could indicate that this core belongs to the $R$. pseudoumbilica Zone. They are, however, here considered to be reworked. Slumps of lower Eocene (NP 13) zeolitic ooze occur in Sections 21 and $2-2$.

\section{Miocene}

The lower Miocene assemblages found in Cores 3 through 6 (Section 1) all lack age-diagnostic sphenoliths, but include Discoaster druggi, Triquetrorhabdulus milowi, and, from Core 5 downward, extremely wellpreserved $T$. carinatus, up to $60 \mu \mathrm{m}$ long; a new Triquetrorhabdulus similar to $T$. striatus, $T$. challengeri, also occurs. These assemblages would indicate the $D$. druggi Zone (NN2), if it were not for the more than sporadic occurrence in most samples of Reticulofenestra abisecta. This species usually terminates below NN2. Discoasters, common in all Miocene samples, indicate a subtropical climate at this site in early Miocene time.

A hiatus in Core 6, Section 1, includes the very lowermost part of the lower Miocene, the whole Oligocene, and the upper Eocene part of the sequence.

\section{Eocene}

The mid-Eocene assemblages in Cores 6 through 10 and the lower Eocene assemblages in Cores 11 through 16 (Section 1) all contain few to common $Z y g$ rhablithus bijugatus. This species is usually found preserved only in samples from shelf areas or depths to about 1000 (?) meters, below which it dissolves (Edwards, personal communication). Another indication of shallow water conditions during the midEocene is the high diversity of the assemblage and the presence of few to common Pontosphaeraceae. Relatively cool climatic conditions during the midEocene are indicated by the estimated discoaster/chiasmolith ratio, which is very low here. 
On the same line of evidence, a warmer climate is indicated during the early Eocene (but see discussion in Perch-Nielsen, this volume). In the lower Eocene samples, diversity of the coccolith assemblage drops, as does the state of preservation.

A hiatus cored in Section 16-2 includes at least NP11 to NP7 (lowermost Eocene and uppermost Paleocene).

\section{Paleocene}

From NP6 (Heliolithus kleinpelli Zone) downward through all remaining coccolith zones to the base of the Danian, an extraordinarily thick sequence of Paleocene (over $120 \mathrm{~m}$ ) was cored continuously, with very high recovery, in Cores 16 through 29. The Cretaceous/Tertiary boundary occurs at Sample $356-29-3,33 \mathrm{~cm}$. The coccolith assemblages are rich and moderately well preserved from Core 17 downward, until the diversity diminishes because fewer species had evolved. Thoracosphaera spp. also becomes increasingly abundant toward the base of the Danian, and in Samples 29-1, $81 \mathrm{~cm}$ and 29-1, $90 \mathrm{~cm}$, it forms a Thoracosphaera-Braarudosphaera chalk. The remainder of the Danian is characterized by the presence of common Thoracosphaera and reworked, very well preserved Maestrichtian coccoliths, including the upper Maestrichtian form Micula mura, which also occurs in the underlying Maestrichtian sequence. The presence of Tertiary planktonic foraminifers of the $G$. eugubina Zone shows this assemblage to belong to the Danian, despite the lack of Biantholithus sparsus, which elsewhere occurs at the very base of this stage. The common occurrence of Thoracosphaera in the Danian, and especially in its lowermost part, has been noted worldwide. The presence of few to common Braarudosphaera in the Danian samples of Core 29 indicates restricted conditions, as found in bays or other nearcontinent or shelf areas. The samples collected from this sequence will provide excellent material for further studies on the evolution of the earliest Tertiary coccoliths. Reworked Late Cretaceous, mainly Maestrichtian, coccoliths are rare to few in most Paleocene samples. Their abundance generally increases downward. In several cores (e.g., 19 and 24) whole layers (slumps) of Maestrichtian chalk occur.

\section{Tertiary/Cretaceous Boundary}

Although very long hiatuses or very compressed Paleocene sequences have been commonly reported from other DSDP sites, and were found at Site 354 on the Ceará Rise and Site 355 in the Brazil Basin, no biostratigraphically recognizable hiatus was found at this site. In the lowermost Tertiary as well as in the uppermost Cretaceous, coccoliths are common and moderately well preserved. The lowermost Tertiary coccolith zone ( $M$. inversus $-B$. sparsus) and the uppermost Cretaceous coccolith zone (M. mura- $N$. frequens) are represented. Reworked Cretaceous coccoliths are, however, present in various amounts in all lower Danian samples.

\section{Upper Cretaceous and Upper Albian}

The upper Cretaceous assemblages are quite diverse but only poorly to moderately well preserved in Cores
29 through 38. In Cores 39 to 41 (Section 4), coccoliths are usually few and poorly preserved. Kamptnerius magnificus, a species indicative of relatively shallow water deposits, is rare in most samples from Cores 29 through 32; below Core 32, it occurs only sporadically. Arkhangelskiella cymbiformis, which was very rare in the open ocean Site 355 in the Brazil Basin, is more common in the Maestrichtian and upper Campanian at this site. It is usually even more common in shelf deposits of chalk, as in the North Sea area. Marthasterites furcatus shows a wide variation of forms, and is most common in Sample 37, CC. It is an easily recognizable marker fossil for the Santonian and Coniacian, and was reported also in the upper Cretaceous on the Falkland Plateau. A late Turonian age is indicated for the lowermost Upper Cretaceous sediments by the presence of Micula staurophora and scarce Gartnerago obliquum and the absence of $M$. furcatus. Below the lithologic change in Section 41-4, infrequent Nannoconus occur in most samples. Because of the very poor preservation of the coccoliths in this interval, age assignment is difficult. The only species classed as few or common in some samples are $W$. barnesae and E. turriseiffeli, two solution-resistant species. Other forms occur rarely and sporadically. The presence of $E$. turriseiffeli suggests a maximum age of late Albian. Lithraphidites alatus, the marker species of the Cenomanian, is absent at this site.

\section{Radiolaria}

The first three cores are barren of siliceous microfossils. Abundant to common radiolarians occur at this site only in the lower Miocene and lower and middle Eocene sediment (Cores 4-15). Radiolarians form a consistent but low-frequency constituent of the coarse fraction in the upper Paleocene sediment (Cores 16 to 23). Except in a few samples from near the bottom of the hole (Samples 42-6, 30-32 cm, and 44, CC) no radiolarians are present in the lower Paleocene and Cretaceous sediments.

Diversity is generally high in the lower Miocene and middle Eocene interval, and decreases downhole in the lower Eocene and upper Paleocene interval. Preservation shows the same trend downhole.

A well-preserved assemblage of the lower Miocene $C$. tetrapora Zone is present in Core 4 and in the bottom and core-catcher sample of Core 2 of Hole 356A. The Lychnocanoma elongata Zone is represented in Core 5, and extends down to Sample $6-1,80 \mathrm{~cm}$. Spines and mesh fragments of orosphaerids, as well as a few callosphaerids, are also present. The total aspect of the assemblages is of a tropical open ocean environment.

The lower Miocene sediment directly overlies middle Eocene sediment containing assemblages of the Podocyrtis mitra Zone, Samples 6-1, $113 \mathrm{~cm}$ and 6-2, 35 $\mathrm{cm}$. The presence of scarce specimens of Sethochytris triconiscus points to the upper part of the zone. Although there is no problem assigning this zone by the standard equatorial radiolarian zonation, a number of species present may indicate a cool- or temperate-water admixed element in the assemblage. An assemblage indicative of the middle Eocene $P$. ampla Zone occurs in Sample 6-2, $35 \mathrm{~cm}$; the rest of Core 6 and Cores 7 
through 9 can be assigned to the Thyrsocyrtis triacantha Zone. Core 10 contains no age-diagnostic radiolarians.

The lower Eocene $P$. striata striata Zone occurs throughout Cores 10 to 13 , and Cores 14 and 15 belong to the Buryella clinata Zone. Again, poor preservation of the assemblages makes it difficult to distinguish between $P$. striata striata, the earliest evolutionary appearance of which defines the base of the $P$. striata striata Zone, and P. striata exquisita.

Although the radiolarians in Sample 16-2, $113 \mathrm{~cm}$ still have an "Eocene look," the radiolarian assemblages from Sample 16-2, $143 \mathrm{~cm}$ to the bottom of Core 23 fit into the unzoned upper Paleocene interval, as described by Foreman (1973). Dictyoceras caia occurs only in Cores 16 and 17; Bekoma divaricata, Buryella tetradica, B. pentadica, Stylosphaera goruna, and Orbula sp. occur consistently but sparsely in all samples down to 23, CC. From the samples examined, it is clear that dissolution and remobilization of biogenic silica has occurred in all samples of early Eocene and late Paleocene age. Recrystallization of silica is evident from Core 24 to Sample 27, CC, where only the general shape of radiolarians, mainly spumellarians, can be recognized.

No radiolarians are present in the sediment below Sample 27, CC except in Sample 42-6, 30-32 cm. The radiolarians in this assemblage are recrystallized, but the following species can be recognized: Lithocampe ananassa, Cyrtocapsa grutterinki, Crucella sp. A., Spongosaturnalis polymorphus, Dictyomitra formosa, Amphipyndax sp. cf. A. stocki, and Patulibracchium cf. $P$. ungulae. The assemblage indicates a late Albian age (RK4/5).

\section{Silicoflagellates}

Bukry (this volume) reports on rich middle Eocene silicoflagellate assemblages from Sections 6-2 through 9-2. Sections 6-2 through 7-1 are assigned to the $D$. hexacantha Zone, Sections 7-3 through 9-1 to the newly defined $D$. spinosa Subzone of the $N$. foliacea Zone, and Section 9-2 to an unnamed subzone of the $N$. foliacea Zone. D. spinosa and Naviculopsis foliacea occur in all four cores, whereas $D$. hexacantha s. str. is restricted to the upper two. The sequence containing silicoflagellates belongs to the upper part of the Nannotetrina fulgens Zone (NP 15) and to the Discoaster bifax Subzone of Bukry (1973).

\section{Diatoms}

Fenner (this volume) reports on diatoms from Cores 5, lower Miocene, through Core 9, middle Eocene. The diatoms in the middle Miocene samples from Core 5 and the top of Section 6-1 are rare and poorly preserved, and permit no age assignment. Below the hiatus in Section 6-1 and through the upper part of Core 9, diatoms are common and well preserved. In the lower part of Core 9 and below, the zeolite content of the sediment increases and diatoms are lacking because of diagenetic dissolution.

Benthic diatoms such as Auliscus sp. and Arachnoidiscus decorus, and species of the genera Rhabdonema,
Raphoneis, Sceptroneis, and Cymatosira (interpreted as littoral diatoms according to their present habitat), occur in all diatom-bearing middle Eocene cores, and indicate relatively shallow conditions or transport from littoral areas into deeper depths. These diatom assemblages also include warm-, temperate-, and coldwater species.

\section{Palynomorphs}

Ionnides and Colin (this volume) have studied palynomorphs from Cores 38 through 41 . They assign a Senonian age, probably younger than late Coniacian, to Core 38, where a sparse and impoverished assemblage including Dinopterygium cladoides, D. acuminatum, and Xenascus ceratoides occurs. Core 39 contains the richest palynomorph assemblage which includes, among others, Dinogymnium, a genus not known to occur before late Coniacian. The palynologic information for Core 40 does not indicate an age more precise than Late Cretaceous; Core 41, virtually devoid of any palynomorphs, shows rare occurrences apparently derived from older sediments.

\section{SEDIMENT ACCUMULATION RATES}

Figure 12 summarizes the sediment accumulation history at Site 356 . Five intervals containing hiatuses or showing extremely slow accumulation rates were cored; two others probably occur between cores.

The accumulation rate was more than $1 \mathrm{~cm} / 1000 \mathrm{yr}$ in the late Albian, when the marly dolomitic limestones and calcareous mudstones with calcareous laminae (Cores 44 to 41 ) were deposited. Nondeposition or erosion followed during the Cenomanian and early Turonian until, later in the late Turonian, black claystones and gray marlstones (Cores 41 through 40 ) were deposited. Similar sediments then formed an intraformational clay-pebble conglomerate (Cores 40 and 39) during the Coniacian. During the latest Coniacian and the Santonian, marly chalks (Cores 38 to 35 ) were deposited at a rate of about 2 to $3 \mathrm{~cm} / 1000 \mathrm{yr}$. Between Cores 35 (upper Santonian) and 36 (Campanian), an interval with slow accumulation or a hiatus occurs, if, as in Figure 12, a long duration is accepted for the Campanian. Accumulation continued at a rate of about $1-2 \mathrm{~cm} / 1000 \mathrm{yr}$ in the late Campanian and the Maestrichtian, and slowed only at the end of the Maestrichtian (Cores 33 to 29).

The thick Paleocene sequence (Cores 29 to 16) of nannofossil and nannofossil-foraminifer chalks was deposited at a rate of about $2 \mathrm{~cm} / 1000 \mathrm{yr}$. Accumulation then ceased for some $5 \mathrm{~m}$.y. during the latest Paleocene and the earliest Eocene $(\sim 57$ to 52 m.y.B.P.), or sediments deposited then were eroded in the earliest Eocene. The early Eocene silicified chalks (Cores 16 to 10) were deposited at the high rate of about $6 \mathrm{~cm} / 1000 \mathrm{yr}$, and are overlain in Core 10 by a thin sequence of siliceous calcareous ooze, which accumulated at the very slow rate of about $0.5 \mathrm{~cm} / 1000$ $\mathrm{yr}$, probably as a result of a high rate of dissolution of biogenic sediments. During the remainder of the middle Eocene (Cores 10-6), accumulation of siliceous 


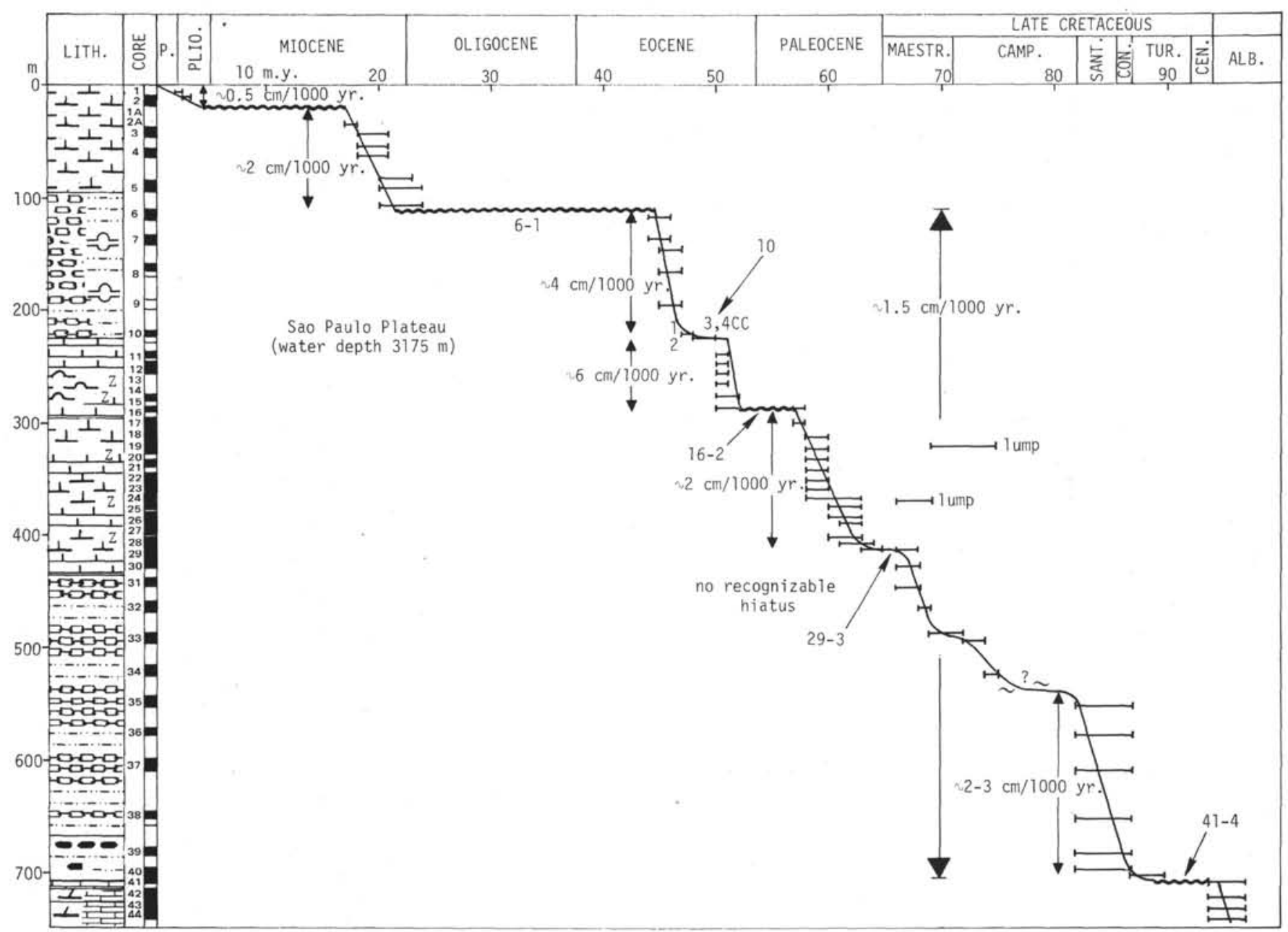

Figure 12. Sedimentation history, Site 356.

calcareous oozes continued at a rate of about 4 $\mathrm{cm} / 1000$ yr until it ceased about $44 \mathrm{~m}$.y. ago. Section 61 includes a hiatus representing some $24 \mathrm{~m} . \mathrm{y}$. and ending about $20 \mathrm{~m} . \mathrm{y}$. ago in the early Miocene, when accumulation resumed.

Lower Miocene nannofossil-foraminifer oozes (Cores 5 to 3, 2A, 1A) were deposited at a rate of 2 $\mathrm{cm} / 1000 \mathrm{yr}$; upper lower to upper Miocene sediments were not recovered. If upper lower to upper Miocene sediments are present between Cores 3 and 2, which were taken with a 19-meter uncored interval between, they would have had to accumulate at an average rate of 0.1 to $0.2 \mathrm{~cm} / 1000 \mathrm{yr}$. This seems unlikely, and we therefore assume that most of the time for which no sediments were recovered is represented by a hiatus. The rate of sediment accumulation in the uppermost part of the sequence was low, in the order of 0.5 $\mathrm{cm} / 1000 \mathrm{yr}$.

Surprisingly many hiatuses or intervals with very slow accumulation occur at this site. But during the periods when sediments were being deposited, accumulation was more rapid than "normal", calcareous ooze accumulation. This may be explained to some extent by the location of Site 356 on the northern flank of a high ridge crest, at the base of which lies a channel almost filled with sediments. The site thus was constantly an area potentially influenced by processes occurring in this valley. Eroding currents probably produced hiatuses or very slow sediment accumulation, while other currents resulted in higher than normal rates of deposition. In fact, the average accumulation rate from the Coniacian through the midEocene is $1.5 \mathrm{~cm} / 1000 \mathrm{yr}$.

A major hiatus was expected at the Cretaceous/Tertiary boundary. The presence at this site of a calcareous Danian succession over 50 meters thick, including the base of the Tertiary, is remarkable, as is the presence of topmost Maestrichtian. Apparently this site was never below the CCD.

Although the occurrence of abundant Braarudosphaera in some Danian samples could be interpreted as indicating shallow water, foraminifers provide evidence indicating water depths close to 1500 meters. A deep CCD at the Cretaceous/Tertiary boundary is indicated by evidence from other DSDP sites, and is discussed in a later chapter (Supko and Perch-Nielsen, this volume).

The slow accumulation in the Campanian and the Cenomanian hiatus correlate well with hiatuses found in the sediment sequences cored on the Falkland Plateau (Leg 36, Site 327). 


\section{CORRELATION OF REFLECTION PROFILE WITH DRILLING RESULTS}

Both the Lamont reference profile (Figure 3) and the approach profile to Site 356 obtained by Glomar Challenger (Figure 5) are of poor quality, so correlation of profiles with the stratigraphic section drilled and cored is difficult at best. Figure 13 contains an interpretive line drawing of the approach profile (Figure 5), ages and lithologies of sedimentary units penetrated, core control, and average interval velocities of sediment as measured with the Hamilton frame onboard ship. Four major reflecting horizons were picked on the approach profile at $0.12,0.28,0.50$, and $0.75 \mathrm{sec}$. These reflectors occur throughout the plateau, and can be correlated from track to track on L-DGO seismic profiles (see Kumar et al., this volume).

At an average velocity of $1.54 \mathrm{~km} / \mathrm{sec}$, the $0.12 \mathrm{sec}$ reflector correlates very well with the boundary, at 93 meters, between the nannofossil and foraminifer oozes of Unit 1 and the siliceous calcareous oozes of Unit 2. The average measured Hamilton frame velocity of 1.62 $\mathrm{km} / \mathrm{sec}$ (Table 5), for cores between the bottom of Unit 1 and the defined lithologic boundary between Units 2 and 3 , calculates to $0.16 \mathrm{sec}$, exactly the measured difference between reflectors 2 and 3 . By similar calculation (see velocities in Table 2), the reflector at $0.5 \mathrm{sec}$ corresponds exactly to the boundary between the nannofossil and nannofossil-foraminifer chalks of Unit 4 and the marly calcareous chalks of Unit 5 . The average core velocity $(2.28 \mathrm{~km} / \mathrm{sec})$ between the top of Unit 5 and the top of the upper Albian limestone sequence (Unit 7) allows calculation of a depth of 716 meters for the $0.75 \mathrm{sec}$ reflector. That is, the $0.75 \mathrm{sec}$ reflector, originally interpreted as regional basement on the basis of Lamont-Doherty reference profiles for the area and the Site 356 approach profile, corresponds to the top of the Albian limestones. Close examination of the approach profile (Figure 5) indicates that true basement may indeed be below the $0.75 \mathrm{sec}$ reflector (dotted line in the drawing of Figure 13).

\section{SUMMARY AND CONCLUSIONS}

Site 356, at the base of the northern flank of the basement ridge at the southeastern edge of the São Paulo Plateau, was drilled and cored to a sediment depth of 741 meters. Igneous basement was not reached; there is reason to believe that true basement lies some hundreds of meters below the total depth reached. The stratigraphic section consists mostly of biogenic sediment, predominantly nannofossil and foraminifer oozes and chalks, with an important siliceous contribution in the Eocene. Seven sedimentary units have been described. Sediment accumulation rates are generally high, on the order of $15 \mathrm{~m} / \mathrm{m}$.y.; several important hiatuses (see Figure 12) indicate periods of nondeposition or removal of sediment by erosional processes.

\section{Age of Basement and Oldest Sediments}

Because the oldest sediments and the crystalline basement could not be reached at this site, we have only an incomplete record with which to interpret the geologic history of this area. We may assume that sediments began to accumulate at this site soon after the opening of the South Atlantic. Estimates for the date of opening of the South Atlantic vary; an estimate of between 120 and $130 \mathrm{~m} . y$. ago may be made on the basis of dating of lavas presumably associated with the rifting (Amaral et al., 1966) and on the basis of ages of recognized magnetic anomalies in the South Atlantic (Larson and Ladd, 1973). The oldest sediments cored at this site are approximately $100 \mathrm{~m} . \mathrm{y}$. old. Hence, the record for approximately the first 20 to $30 \mathrm{~m}$.y. of the geologic history of this area was not sampled. But this estimate does not take into account the time needed to create the oceanic crust between this site and the ocean-continent boundary off the Brazilian continent; so the date for the opening of the South Atlantic is only an estimate of the oldest possible basement age at this site.

We can use the age of the salt to guess at the youngest possible age for the basement. The salt beds in the South Atlantic are Aptian-Albian in age (Asmus and Ponte, 1973; Brink, 1974). The ridge at the southern edge of the São Paulo Plateau, together with the Walvis Ridge, appears to have acted as a barrier to circulation during formation of the salt (Leyden and Nunes, 1972; Leyden et al., in press). The São Paulo ridge must have formed the barrier during deposition of the salt layer, which contains diapiric structures about $100 \mathrm{~km}$ to the northwest. Hence the crust at Site 356 must have been created prior to the late Aptian (approximately 110 m.y.), and the basement age must be somewhere between 110 and $127 \mathrm{~m} . \mathrm{y}$. This estimate is supported by the age of the crust underlying Site 364 , which is near the seaward edge of the salt layer on the African side. The crust underlying Site 364 is of anomaly M-4 age (116 m.y., Larson and Pitman, 1972; Bolli, Ryan, et al., 1975). Site 356 is on the Brazilian margin in a situation somewhat analogous to that of Site 364 on the Angolan margin.

Our estimates assume that the crust under the São Paulo Plateau is oceanic. The fit of South America with Africa (Bullard et al., 1965) in this area, and the geophysical data from the São Paulo Plateau (Leyden et al., 1971) support this assumption. Leyden (in press), however, has suggested the existence of a "transitional oceanic" crust, underlying the plateau, presumably created by intrusion of mafic material into tensional fissures in continental crust, and not from a central spreading ridge.

Sediment thickness under the plateau ranges up to 4 $\mathrm{km}$ (Francisconi and Kowsmann, in press). Presumably the São Paulo ridge, which formed during or immediately after the opening of the South Atlantic, acted as a barrier, so sediments supplied from the Brazilian continent could not escape into the open ocean. The sedimentary sequence underlying the São Paulo Plateau appears to be the offshore equivalent of the sequence in the Santos Basin, which underlies the Brazilian shelf between $23^{\circ}$ and $28^{\circ}$ latitudes (Kumar et al., this volume; Asmus and Ponte, 1973; Asmus, 1975; Ponte and Asmus, in press).

\section{Early Cretaceous History}

At the time the oldest unit cored at this site (Unit 7) was emplaced, salt deposition had ceased. Since salt 


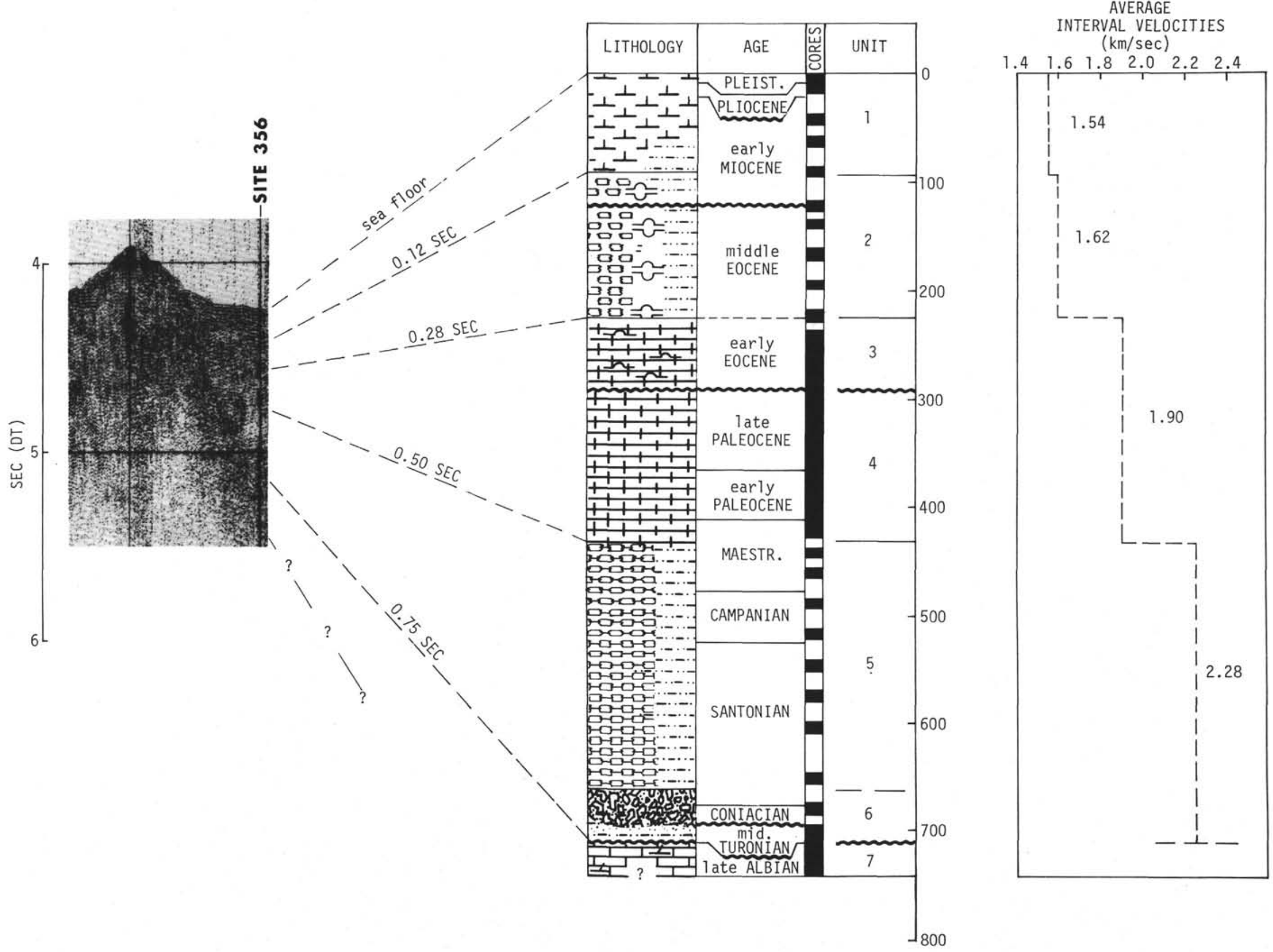


deposition had ceased by the end of the Aptian (Asmus and Ponte, 1973; Brink, 1974), and Unit 7 is of late Albian age, the sampled part of Unit 7 represents conditions that existed approximately 6 to $8 \mathrm{~m}$.y. after the end of salt deposition. Sediments equivalent in age and composition to Unit 7 were cored at Site 364 in the Angola Basin off the West African margin. At Site 364, upper Albian limestones overlie upper Aptian to lower Albian sapropelic shales and marly dolomitic limestones, which overlie the salt (Bolli, Ryan, et al., 1975).

The marly dolomitic limestones of Unit 7 were deposited in an open marine environment. Hence the dolomitic limestones of the kind represented in Unit 7 were deposited throughout the South Atlantic when open marine conditions were established at the end of salt deposition. The bulk of the sediment of Unit 7 is terrigenous-calcareous in composition. Therefore, the abundant white laminae of pure calcium carbonate must be the result of some process which removed the terrigenous clays and allowed calcium carbonate to concentrate in thin discrete layers. The thickness, abundance, and ungraded nature of these laminae suggest that bottom currents may have formed them by removing the terrigenous clays. Short-term rapid accumulation of pelagic calcareous material or calcareous turbidites may produce such laminae, but in the case of pelagic calcareous material one would expect the upper or lower contacts to be gradational, and in the case of calcareous turbidites one would expect graded bedding and gradational upper contacts.

The coarse sand-sized graded layers of Unit 7 probably represent turbidites which originated at the Brazilian margin. The presence of quartz in the coarse graded layers supports this suggestion. Thus, eroding, as well as depositing, currents were operating in the area at the time Unit 7 was deposited.

The benthic foraminifers imply a depth of approximately 1000 meters at the site when Unit 7 was deposited. So the basin was at least that deep 6 to 8 m.y. after salt deposition had ceased. Assuming that conditions remained more or less unchanged, one may conclude that the basin was several hundred meters deep when salt deposition ended in the South Atlantic. On the other hand, if the basin was only tens of meters deep at the end of salt deposition, a slow subsidence of only $0.2 \mathrm{~mm} / \mathrm{yr}$ ( $1000 \mathrm{~m}$ in $5 \mathrm{~m}$.y.) would be sufficient to create a depth of 1000 meters during the deposition of Unit 7. Aptian sediments at Site 361 and 364 (Walvis Ridge and Angola Basin, respectively) indicate, however, that the eastern basin was a few hundred meters deep at the end of salt deposition (Bolli, Ryan, et al., 1975). By analogy, the basin at Site 356 was most probably also a few hundred meters deep when salt deposition ended in late Aptian. The basin then subsided.

Deposition of salt took place entirely within the Aptian, probably within the late Aptian. Even if we assume that the entire period of salt deposition was only $3 \mathrm{~m} . \mathrm{y}$. , a subsidence of $1 \mathrm{~mm} / \mathrm{yr}$ could accommodate deposition of the $3 \mathrm{~km}$ of salts (Bolli, Ryan, et al., 1975; Leyden et al., 1971) that occur on both sides of the Atlantic. Although this rate of subsidence is not excessive, analogy with other salts suggests that deposition of salt occurs at such a high rate that crustal subsidence cannot keep pace with it (Faure, in press; Ryan, personal communication, 1975). Such indirect evidence thus favors an interpretation in which the basin is considered to have been 3 to $4 \mathrm{~km}$ deep before the salts began to accumulate, and almost filled to a depth of less than $1 \mathrm{~km}$ after the salt deposition had ceased (Bolli, Ryan, et al., 1975).

\section{Coniacian-Turonian Mudstones}

Cores 39,40 , and 41 contain repeating sequences of black carbonaceous mudstones (containing pyrite) and medium gray and greenish-gray layers of nannofossil marl. The black carbonaceous layers indicate reducing conditions, whereas the light gray layers were deposited under aerobic conditions (presence of benthic foraminifers and burrows). Two alternative hypotheses warrant consideration: that the black mudstone layers were periodically introduced from outside, or the redox state of the depositional site changed periodically.

If the pyritiferous mudstones were introduced periodically into an otherwise aerobic area of deposition, one would expect to see evidence of resedimentation. The conglomerate-to-dark clay sequence of Core 39 does indeed appear to have been transported through sediment-gravity flows. The sediments of Core 41 , by contrast, show gradational contacts and hence do not suggest resedimentation of the sediments contained in black layers. Gradational contacts imply in situ deposition. The high organic content and pyrite in the black layers indicate reducing conditions, and the presence of benthic foraminifers in the gray layers and burrowing indicate bottom life and therefore oxidizing conditions. Conditions of cyclic change from oxidizing to reducing conditions could explain this. The overall low accumulation rate of Unit 6 favors formation in situ of a major part of the sediments in this unit, rather than their introduction from outside. The graded conglomerate-to-clay sequence may represent local resedimentation in an already anaerobic basin.

Black sapropelic sediments were noted in the Cretaceous and Paleocene at several Leg 14 sites (see Berger and von Rad, 1972); they included a welldeveloped rhythmic sequence of black pyrite-bearing carbonaceous shales and gray-green dolomitic lutites. The authors explained the presence of the dark layers as a consequence of redeposition processes, although the preponderance of carbonaceous sediments in the Cretaceous North Atlantic (and Caribbean, for discussion see Saunders et al., 1973, and their fig. 11) sediments makes periodic basin-wide stagnation an alternative possibility.

Although the dark layers of Core 39 show evidence of resedimentation (conglomeratic nature, grading on a large scale), those of Cores 40 and 41 have gradational contacts at top and bottom. Thus, while some of the dark layers may have been emplaced by resedimentation processes, at least some seem to have formed in situ during periods when reducing conditions prevailed. The prominent basement ridge nearby leads one to 
suspect periodic reduced (or cessation of) deep circulation by relative fluctuations of the ridge topography and/or eustatic sea level.

\section{Post-Coniacian History}

Oxidizing conditions existed in the basin during deposition of the Santonian to Maestrichtian marly chalks (Unit 5). The sediments are fairly well burrowed, indicating that bottom-dwelling organisms existed in the area. The significance of the presence of Inoceramus fragments in terms of environment of deposition is unknown (see Thiede and Dinkelman, this volume). The terrigenous components were being supplied from the margin, whereas the calcareous component is pelagic-biogenic in nature. Occasional gravitycontrolled bottom flows deposited coarse graded layers. It is significant that conglomerates and coarsegrained sandstones were being deposited in the Santos basin during the time the marly chalks were deposited.

Unit 4 consists of relatively pure nannofossil and nannofossil-foraminifer chalks, only locally marly, of late Maestrichtian to late Paleocene age. The Cretaceous/Tertiary boundary at this site is represented by continuous deposition. Since benthic foraminifers indicate a water depth of 1000 meters at this time, it is necessary to look more critically at Worsley's (1974) hypothesis that the CCD in the open ocean had shallowed into the photic zone, thereby precluding abundant deposition of carbonates at any great depth. Indeed, a very thick (approximately $50 \mathrm{~m}$ ) Danian section was recovered, perhaps the best and oldest Danian section yet recovered from the deep sea.

The supply of terrigenous sediment to the area was cut off during the period when the Maestrichtian-upper Paleocene chalks were deposited. A Paleocene hiatus occurs in the Santos basin, suggesting that supply of coarse terrigenous material was minimal during that time (Leyden et al., in press; Asmus, 1975). Apparently because no terrigenous material was supplied to the basin, the resulting sediments in the distal part of the basin are pelagic-calcareous in nature. Cretaceous slump material present in Paleocene Cores 19, 24, and 25 must have come from the nearby São Paulo ridge, because supply of terrigenous material from South America was only minimal. Moreover, the slumped material is different from the sediments being deposited in the basin at that time. The slump material is almost entirely pelagic, which further supports the argument that it came from the ridge.

The Eocene was a time of incursion of cooler waters and of important contributions of siliceous fossil tests to the sediment. In contrast to the foraminifers and ostracodes (Benson, this volume), which indicate deposition at bathyal depths, nannofossils indicate water depths possibly less than 1000 meters. Benthic diatoms (Fenner, this volume) also indicate shallow water, but the high Eocene sedimentation rate of 4 $\mathrm{m} / 10 \mathrm{~m} . \mathrm{y}$. may reflect transport into the basin from shallow water areas. Evidence from all three major fossil groups (nannofossils, foraminifers, and radiolarians) indicates cool subtropical conditions, which are typical of middle latitudes at this time (Premoli-Silva, in preparation). The Eocene sediments are divided into two lithologic units. The siliceous component of the middle Eocene Unit 2 is composed of the tests of radiolarians and frustules of diatoms. In the lower Eocene Unit 3, the silica forms a cement for the (silicified) chalks. Because the transition from Unit 2 to Unit 3 is very sharp, it is probable that silica was remobilized as cement by chemical changes in the pore water, and not solely by overburden and burial. Preservation of siliceous biogenic components progressively deteriorates from Unit 2 down into Unit 3.

Major hiatuses cover the time span from late middle Eocene through Oligocene and late early Miocene through Pliocene. A correlation with high latitude paleotemperature curves (Shackleton and Kennett, 1975 ) indicates these to be the coldest periods of the late Paleogene and pre-Pleistocene Cenozoic.

The Neogene sediments of Unit 1 are almost entirely pelagic; hence terrigenous material has not reached this site since the early Miocene. Continued slumping from the nearby São Paulo ridge is indicated by the presence of manganese crust and zeolitic foraminifer-nannofossil ooze of Eocene age. Early Miocene vulcanism produced an ash layer in Core 4 . The volcanic ash appears to be from a continental source (interpreted silica content $60 \%$ ), but no continental vulcanism of Miocene age is known from the Brazilian continent at this time. The volcanic islands of the ColumbiaTrinidade seamount chain (see base map for Site 355) to the north of Site 356 have, however, been active as recently as Pliocene time (Cordani, 1970).

Figure 14 summarizes the possible sources of sediments for various units identified at Site 356 .

\section{REFERENCES}

Amaral, G., Cordani, U.G., Kawashita, K., and Reynolds, J.H., 1966. Potassium-argon dates of basaltic rocks from southern Brazil: Geochim. Cosmochim. Acta, v. 31, p. $117-142$.

Asmus, H.E., 1975. Controle Estrutural da deposicao Mesozoica nas bacias da margem continental Brasileira: Rev. Brasileira Geocienc., São Paulo, Brazil, v. 5, p. 160175.

Asmus, H.E. and Ponte, F.D., 1973. The Brazilian marginal basins: In Nairn, A.E.M. and Stehli, F.G. (Eds.), The ocean basins and margins, v. 1, the South Atlantic: New York (Plenum Press), p. 87-133.

Berger, W.H. and von Rad, U., 1972. Cretaceous and Cenozoic sediments from the Atlantic Ocean. In Hayes, D.E., Pimm, A.C. et al., Initial Reports of the Deep Sea Drilling Project, Volume 14: Washington (U.S. Government Printing Office), p. 787-954.

Bolli, H.M., Ryan, W.B.F., et al., 1975. Basins and margins of the eastern South Atlantic: Geotimes, v. 20, p. 22-24.

Brink, A.H., 1974. Petroleum geology of Gabon basin: Am. Assoc. Petrol. Geol. Bull., v. 58, p. 216-235.

Bukry, D., 1973. Low latitude coccolith biostratigraphic zonation. In Edgar, N.T., Saunders, J.B., et al., Initial Reports of the Deep Sea Drilling Project, Volume 15: Washington (U.S. Government Printing Office), p. 685703.

Bullard, E.C., Everett, J.E., and Smith, A.F., 1965. The fit of the continents around the Atlantic. In Blackett, P.M.S., Bullard, E., and Runcorn, S.K. (Eds.), A symposium on 


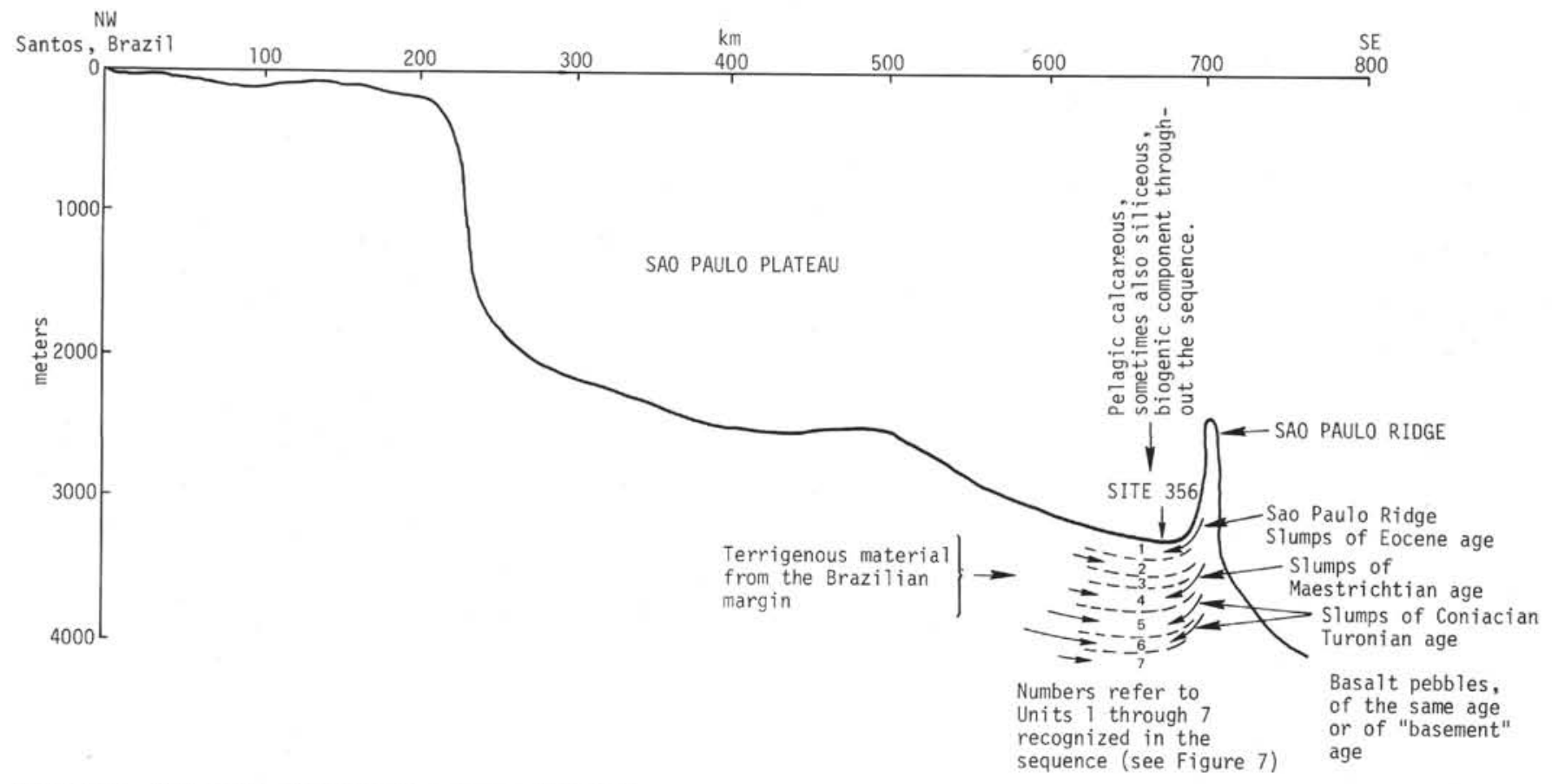

Figure 14. Summary of sediment sources, Site 356.

continental drift: Phil. Trans. Roy. Soc. London, ser. A, v. 258 , p. 41-51.

Cordani, U.G., 1970. Idade do vulcanismo no Atlantico Sul: Bol. Instituto do Geociencias e Astronomia, Universidade de São Paulo, v. i, p. 9-75.

Faure, H., in press. Vertical evolution of continental margins. In Almeida, F.F.M. de (Ed.), Symposium on Atlantic-type continental margins: São Paulo, Brazil, 13-18 Oct. 1975.

Foreman, H., 1973. Radiolaria of Leg 10 with systematics and ranges for the families Amphipyndacidae, Artostrobiidae, and Theoperidae. In Worzel, J.L., Bryant, W., et al., Initial Reports of the Deep Sea Drilling Project, Volume 10: Washington (U.S. Government Printing Office), p. 407-474.

Francisconi, O. and Kowsmann, R. O., in press. Preliminary structural study of the South Brazilian continental margin. In Almeida, F.F.M. de (Ed.), Symposium on Atlantic-type continental margins: São Paulo, Brazil, 13-18 Oct. 1975.

Larson, R.L. and Ladd, J.W., 1973. Evidence for the opening of the South Atlantic in the Early Cretaceous: Nature, v. 246 , p. $209-212$.

Larson, R.L. and Pitman, W.C., III, 1972. World-wide correlation of Mesozoic magnetic anomalies and its implications: Geol. Soc. Am. Bull., v. 83, p. 3645-3662.

Leyden, R., in press. Salt distribution and crustal models for the eastern Brazilian margin. In Almeida, F.F.M. de (Ed.), Symposium on Atlantic-type continental margins: São Paulo, Brazil, 13-18 Oct. 1975.

Leyden, R. and Nunes, J.R., 1972. Diapiric structures offshore southern Brazil: 26th Brazilian Geol. Cong. Trans., p. 45-50.

Leyden, R., Ludwig, W.J., and Ewing, M., 1971. Structure of the continental margin off Punta del Este, Uruguay and Rio de Janeiro, Brazil: Am. Assoc. Petrol. Geol. Bull., v. 55 , p. $2161-2173$.

Leyden, R., Bryan, G., and Ewing, M. 1972. Geophysical reconnaissance on the African Shelf: 2, Margin sediments from Gulf of Guinea to the Walvis Ridge: Am. Assoc. Petrol. Geol. Bull., v. 56, p. 682-693.

Leyden, R., Asmus, H., Bryan, G., and Zembruscki, S., in press. Diapiric structures in the South Atlantic: Am. Assoc. Petrol. Geol. Bull., v. 60.

Pautot, G., Renard, V., Daniel, J., and Dupont, J., 1973. Morphology, limits, origin, and age of salt layer along South Atlantic African margin: Am. Assoc. Petrol. Geol. Bull., v. 57, p. 1658-1672.

Ponte, F.C. and Asmus, H.E., in press. The Brazilian marginal basins: Current state of knowledge. In Almeida, F.F.M. de (Ed.), Symposium on Atlantic-type continental margins: São Paulo, Brazil, 13-18 Oct. 1975.

Premoli-Silva, I. and Bolli, H.M., 1973. Late Cretaceous to Eocene planktonic foraminifera and stratigraphy of Leg 15 sites in the Caribbean Sea. In Edgar, N.T., Saunders, J. B., et al., Initial Reports of the Deep Sea Drilling Project. Volume 15: Washington (U.S. Government Printing Office), p. 499-527.

Reyment, R.A. and Tait, E.A., 1972. Biostratigraphical dating of the early history of the South Atlantic Ocean: Phil. Trans. Roy. Soc. London, v. 264, p. 55-95.

Saunders, J.B., Edgar, N.T., Donnelly, T.W., and Hay, W.W., 1973. Cruise synthesis. In Edgar, N.T., Saunders, J.B. et al., Initial Reports of the Deep Sea Drilling Project, Volume 15: Washington (U.S. Government Printing Office), p. 1077-1111.

Shackleton, N.J. and Kennett, J.P., 1975. Late Cenozoic oxygen and carbon isotopic changes in DSDP Site 284; implications for glacial history of the northern hemisphere and of Antarctica. In Kennett, J.P., Houtz, R.E., et al., Initial Reports of the Deep Sea Drilling Project, Volume 29: Washington (U.S. Government Printing Office), p. 801-808.

Worsley, T., 1974. The Cretaceous-Tertiary boundary event in the ocean. In Hay, W.W. (Ed.), Studies in paleooceanography: Soc. Econ. Paleontol. Mineral., Spec. Pub. 20, p. 94-125. 
APPENDIX A

Smear-slide Summary

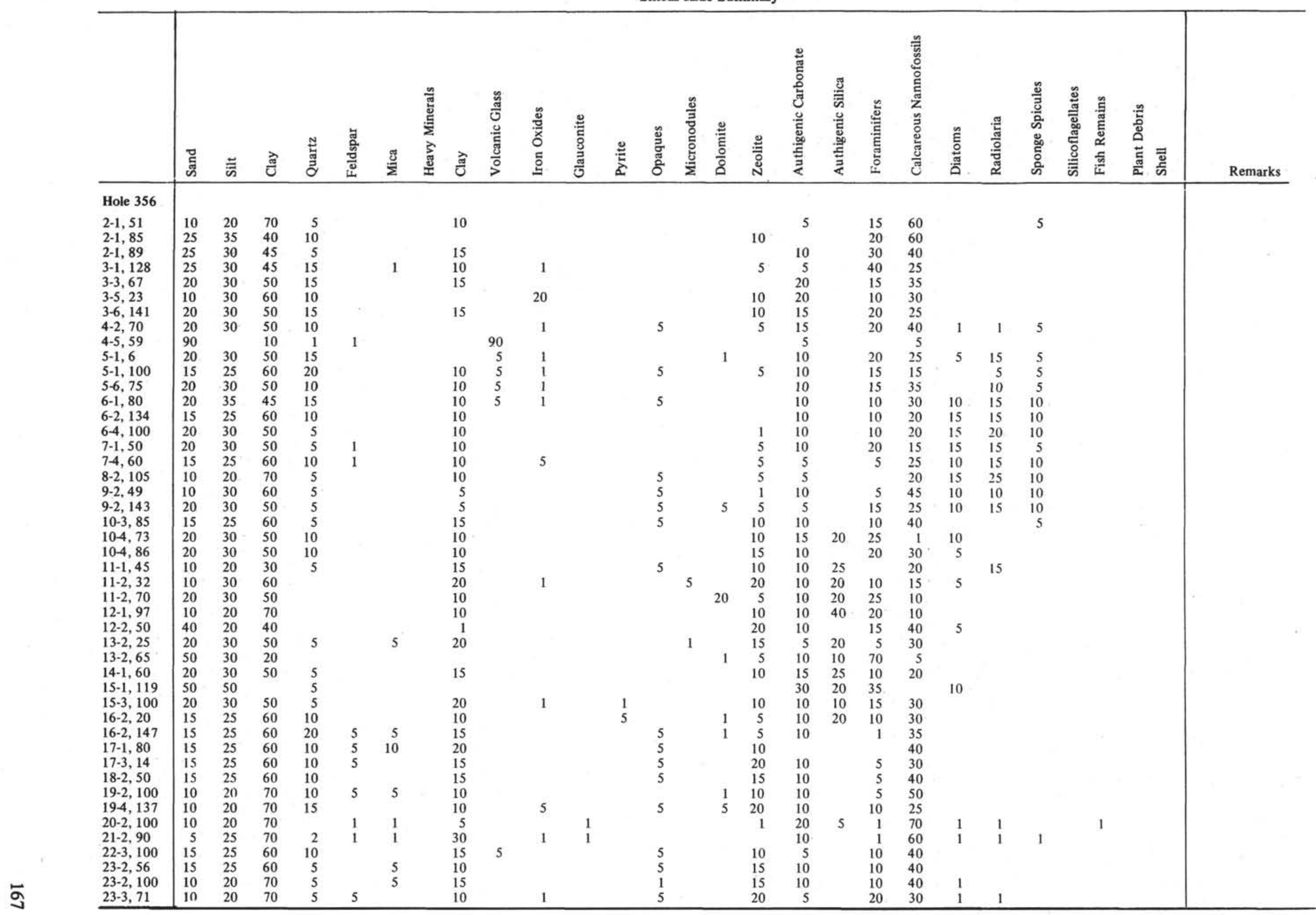

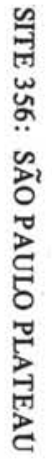




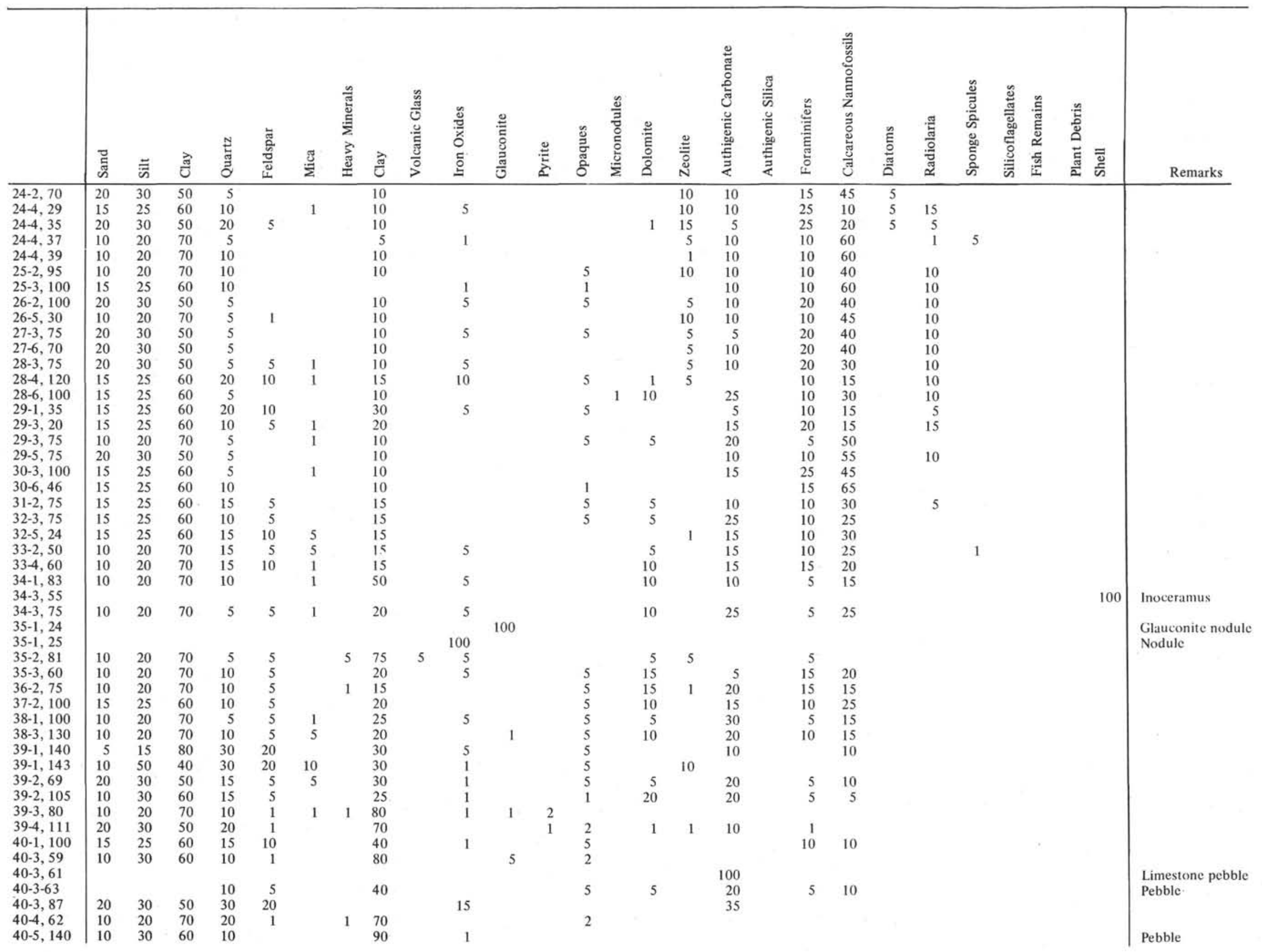




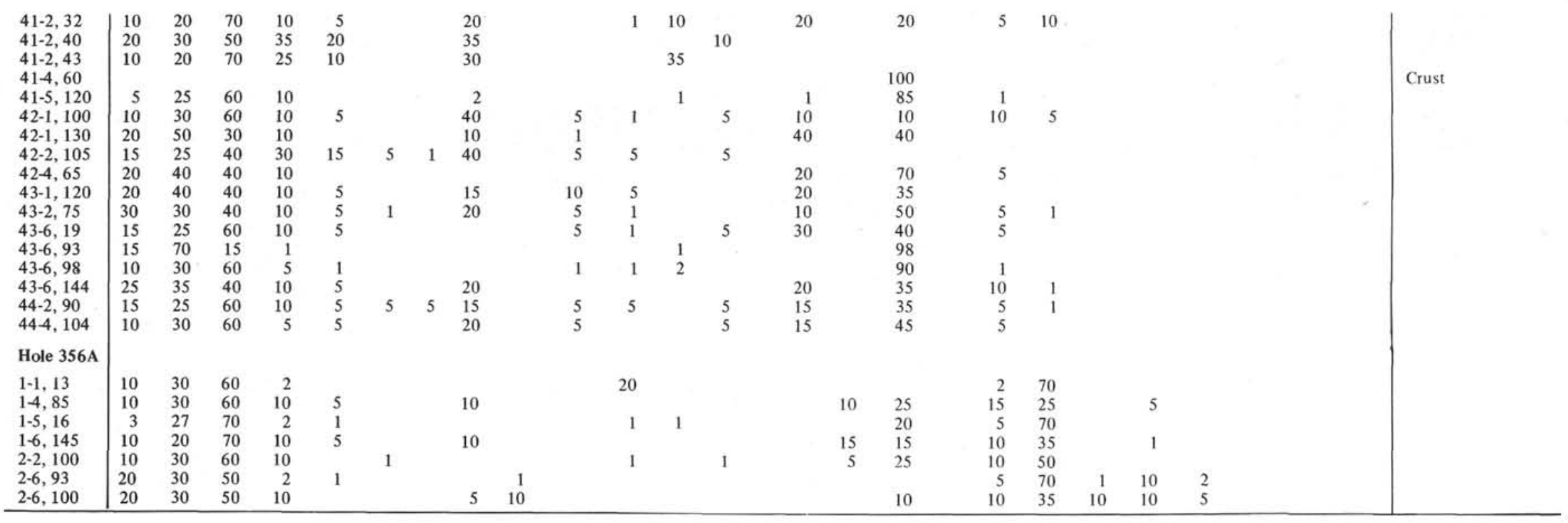


APPENDIX B

Carbonate and Quartz Determinations Leg 39

\begin{tabular}{|c|c|c|c|c|c|}
\hline Section & $\begin{array}{c}\text { Sediment } \\
\text { Depth }(\mathrm{cm})\end{array}$ & $\mathrm{CaCO}_{3}(\%)$ & Org (\%) & $\begin{array}{c}\text { Total } \\
\text { Carb (\%) }\end{array}$ & Qtz (\%) \\
\hline $2-2$ & 1110 & 82.01 & 0.05 & 9.90 & \\
\hline $2-2$ & 1151 & 72.79 & 0.69 & 9.42 & 12.73 \\
\hline $2-2$ & 1195 & 50.71 & 0.94 & 7.03 & 8.86 \\
\hline $3-2$ & 4068 & 49.55 & 0.07 & 6.02 & \\
\hline $3-4$ & 4355 & 41.85 & 1.01 & 6.03 & 11.25 \\
\hline $3-6$ & 4692 & 44.72 & 0.70 & 6.07 & 14.81 \\
\hline 43 & 6078 & 31.93 & 1.37 & 5.20 & 10.32 \\
\hline $4-3$ & 6086 & 43.68 & 0.17 & 5.42 & \\
\hline $4-5$ & 6359 & 10.73 & 0.92 & 2.21 & 7.57 \\
\hline 5-4 & 9033 & 43.48 & 1.12 & 6.34 & 16.63 \\
\hline $5-5$ & 9270 & 46.17 & 0.07 & 5.61 & \\
\hline $6-1$ & 11466 & 17.03 & 1.39 & 3.43 & 15.91 \\
\hline $6-4$ & 11860 & 28.55 & 0.15 & 3.58 & \\
\hline $6-4$ & 11910 & 19.61 & 1.03 & 3.38 & 5.57 \\
\hline $6-6$ & 12295 & 36.95 & 0.52 & 4.99 & 7.78 \\
\hline $7-1$ & 13350 & 54.70 & 0.54 & 7.11 & 8.53 \\
\hline $7-4$ & 13760 & 17.89 & 0.71 & 2.86 & 6.96 \\
\hline $7-4$ & 13770 & 21.19 & 0.23 & 2.77 & \\
\hline $8-2$ & 16380 & 26.58 & 0.54 & 3.73 & 4.73 \\
\hline $8-2$ & 16390 & 34.44 & 0.15 & 4.28 & \\
\hline $9-2$ & 19254 & 40.68 & 0.17 & 5.06 & \\
\hline $9-2$ & 19270 & 36.51 & 0.67 & 5.05 & 4.56 \\
\hline $10-3$ & 22186 & 24.07 & 0.85 & 3.74 & 4.91 \\
\hline $10-3$ & 22192 & 31.52 & 0.17 & 3.95 & \\
\hline $10-4$ & 22391 & 48.94 & 0.55 & 6.43 & 7.24 \\
\hline $11-1$ & 23777 & 26.32 & 0.18 & 3.34 & \\
\hline $11-1$ & 23783 & 17.90 & 0.58 & 2.73 & 4.44 \\
\hline $12-1$ & 24735 & 22.92 & 0.15 & 2.90 & \\
\hline $12-2$ & 24894 & 22.66 & 0.57 & 3.29 & 4.21 \\
\hline $13-2$ & 25856 & 15.79 & 0.55 & 2.45 & 3.79 \\
\hline 141 & 26637 & 20.59 & 0.11 & 2.58 & \\
\hline $15-1$ & 27669 & 42.70 & 0.86 & 5.98 & 3.69 \\
\hline $16-1$ & 28596 & 23.90 & 0.15 & 3.02 & \\
\hline $16-2$ & 28702 & 20.91 & 0.49 & 3.00 & 5.09 \\
\hline $17-3$ & 29800 & 14.90 & 0.39 & 2.17 & \\
\hline $17-4$ & 29970 & 13.22 & 0.61 & 2.20 & 7.49 \\
\hline $18-2$ & 30660 & 17.57 & 0.69 & 2.80 & 8.27 \\
\hline $19-4$ & 31840 & 43.06 & 0.07 & 5.24 & \\
\hline $19-4$ & 31840 & 16.47 & 0.70 & 2.68 & 5.93 \\
\hline $20-2$ & 32455 & 26.19 & 0.68 & 3.82 & 6.81 \\
\hline $22-1$ & 34330 & 32.08 & 0.57 & 4.42 & 6.20 \\
\hline $23-2$ & 35350 & 30.17 & 0.18 & 3.80 & \\
\hline $23-3$ & 35408 & 31.13 & 0.62 & 4.36 & 5.98 \\
\hline $24-2$ & 36396 & 48.32 & 0.41 & 6.21 & 3.89 \\
\hline $24-3$ & 36445 & 47.62 & 0.21 & 5.93 & \\
\hline $24-3$ & 36500 & 19.73 & 0.10 & 2.47 & \\
\hline $24-4$ & 36584 & 66.86 & 0.09 & 8.11 & \\
\hline $24-5$ & 36820 & 43.12 & 0.11 & 5.29 & \\
\hline $24-6$ & 36940 & 44.19 & 0.58 & 5.88 & 5.27 \\
\hline $25-2$ & 37198 & 45.43 & 0.82 & 6.28 & 7.04 \\
\hline $25-5$ & 37730 & 49.05 & 0.57 & 6.46 & 5.64 \\
\hline $26-2$ & 38168 & 40.92 & 0.67 & 5.58 & 8.96 \\
\hline $26-2$ & 38258 & 48.70 & 0.17 & 6.02 & \\
\hline $27-1$ & 39085 & 40.24 & 0.69 & 5.55 & 6.71 \\
\hline $28-1$ & 40034 & 26.18 & 0.55 & 3.70 & 7.40 \\
\hline $29-1$ & 40930 & 40.30 & 0.85 & 5.81 & 8.64 \\
\hline $29-2$ & 41090 & 34.24 & 0.18 & 4.29 & \\
\hline $29-3$ & 41230 & 57.57 & 0.34 & 7.25 & 9.76 \\
\hline $29-3$ & 41299 & 69.13 & 0.08 & 8.38 & \\
\hline $29-4$ & 41344 & 46.76 & 0.07 & 5.68 & \\
\hline $29-6$ & 41660 & 68.04 & 0.08 & 8.25 & \\
\hline $30-6$ & 42597 & 49.26 & 0.53 & 6.44 & 10.69 \\
\hline $31-5$ & 44380 & 63.00 & 0.00 & 7.56 & 8.76 \\
\hline $31-5$ & 44396 & 66.51 & 0.07 & 8.05 & \\
\hline $32-3$ & 45931 & 40.12 & 0.07 & 4.89 & 11.37 \\
\hline $33-2$ & 48655 & 36.42 & 0.06 & 4.43 & \\
\hline
\end{tabular}


APPENDIX B - Continued

\begin{tabular}{|c|c|c|c|c|c|}
\hline Section & $\begin{array}{c}\text { Sediment } \\
\text { Depth }(\mathrm{cm})\end{array}$ & $\mathrm{CaCO}_{3}(\%)$ & Org (\%) & $\begin{array}{c}\text { Total } \\
\text { Carb (\%) }\end{array}$ & Qtz (\%) \\
\hline $33-2$ & 48680 & 40.19 & 0.02 & 4,85 & 10.15 \\
\hline 342 & 51520 & 41.73 & 0.09 & 5.10 & \\
\hline 342 & 51520 & 38.89 & 0.17 & 4.84 & 13.27 \\
\hline $35-3$ & 54480 & 37.22 & 0.10 & 4.57 & 15.13 \\
\hline $35-3$ & 54550 & 30.60 & 0.12 & 3.79 & \\
\hline $36-1$ & 57021 & 35.36 & 0.29 & 4.54 & 15.14 \\
\hline $36-1$ & 57130 & 44.29 & 0.13 & 5.45 & \\
\hline $37-4$ & 60380 & 40.77 & 0.19 & 5.08 & 14.95 \\
\hline $37-4$ & 60383 & 47.76 & 0.09 & 5.82 & \\
\hline $38-3$ & 64959 & 34.63 & 0.29 & 4.45 & 12.62 \\
\hline $38-3$ & 64986 & 46.41 & 0.09 & 5.66 & \\
\hline $39-2$ & 67600 & 63.18 & 0.22 & 7.80 & 8.75 \\
\hline $39-4$ & 68005 & 35.75 & 0.36 & 4.65 & 13.56 \\
\hline $39-5$ & 68071 & 0.36 & 1.82 & 1.86 & \\
\hline $39-5$ & 68125 & 5.29 & 5.311 & 5.95 & 13.08 \\
\hline $40-1$ & 69427 & 55.70 & 0.38 & 7.07 & 2.29 \\
\hline $40-5$ & 70007 & 45.22 & 0.10 & 5.53 & \\
\hline $40-6$ & 70154 & 10.13 & 3.03 & 5.45 & 16.64 \\
\hline $40-6$ & 70155 & 13.50 & 2.46 & 4.08 & \\
\hline $41-2$ & 70484 & 49.04 & 0.31 & 6.19 & 17.32 \\
\hline $41-2$ & 70524 & 3.81 & 4.84 & 5.23 & \\
\hline $41-3$ & 70710 & 14.98 & 4.84 & 6.64 & 16.47 \\
\hline $41-4$ & 70804 & 32.43 & 11.97 & 15.86 & 10.41 \\
\hline $41-4$ & 70806 & 38.68 & 10.45 & 15.14 & 8.90 \\
\hline $41-5$ & 71048 & 51.81 & 0.64 & 6.86 & \\
\hline $42-3$ & 71696 & 20.08 & 0.102 & 2.51 & \\
\hline $43-4$ & 72657 & 33.09 & 0.12 & 4.09 & \\
\hline $43-5$ & 72897 & 28.04 & 0.08 & 3.45 & \\
\hline $43-5$ & 72897 & 20.51 & 0.22 & 2.68 & \\
\hline $44-4$ & 73700 & 43.61 & 0.10 & 5.33 & \\
\hline $1-4$ & 2430 & 32.47 & 0.08 & 3.97 & 12.15 \\
\hline $1-4$ & 2436 & 27.78 & 0.81 & 4.14 & 12.15 \\
\hline $1-4$ & 2483 & 28.97 & 0.53 & 4.01 & 12.81 \\
\hline $1-5$ & 2525 & 36.63 & 0.59 & 4.99 & 15.29 \\
\hline $1-5$ & 2595 & 41.52 & 0.11 & 5.09 & \\
\hline $1-6$ & 2795 & 39.16 & 0.86 & 5.56 & 15.52 \\
\hline $2-5$ & 3462 & 45.36 & 0.14 & 5.59 & \\
\hline $2-5$ & 3567 & 34.28 & 0.88 & 4.99 & 16.74 \\
\hline
\end{tabular}




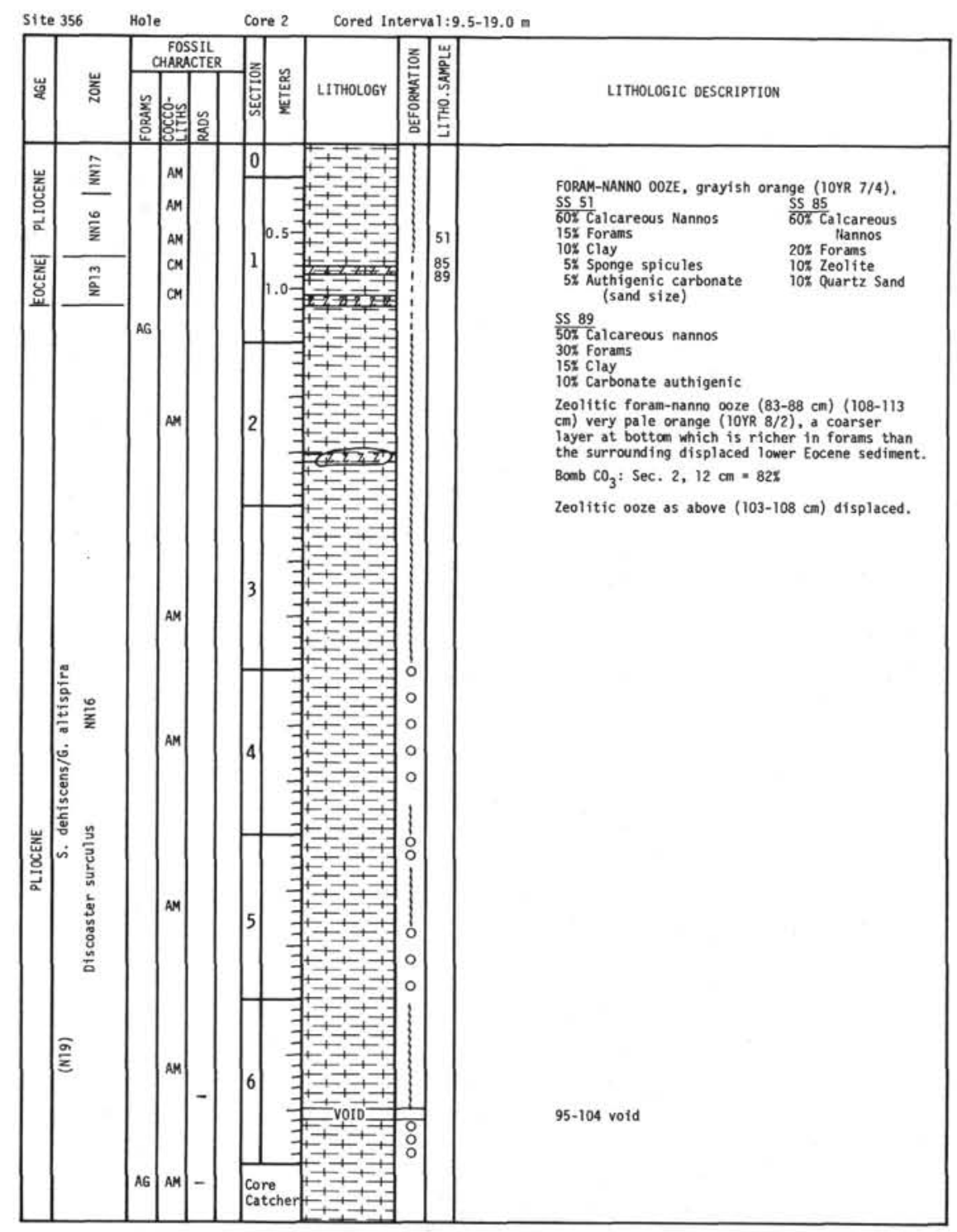

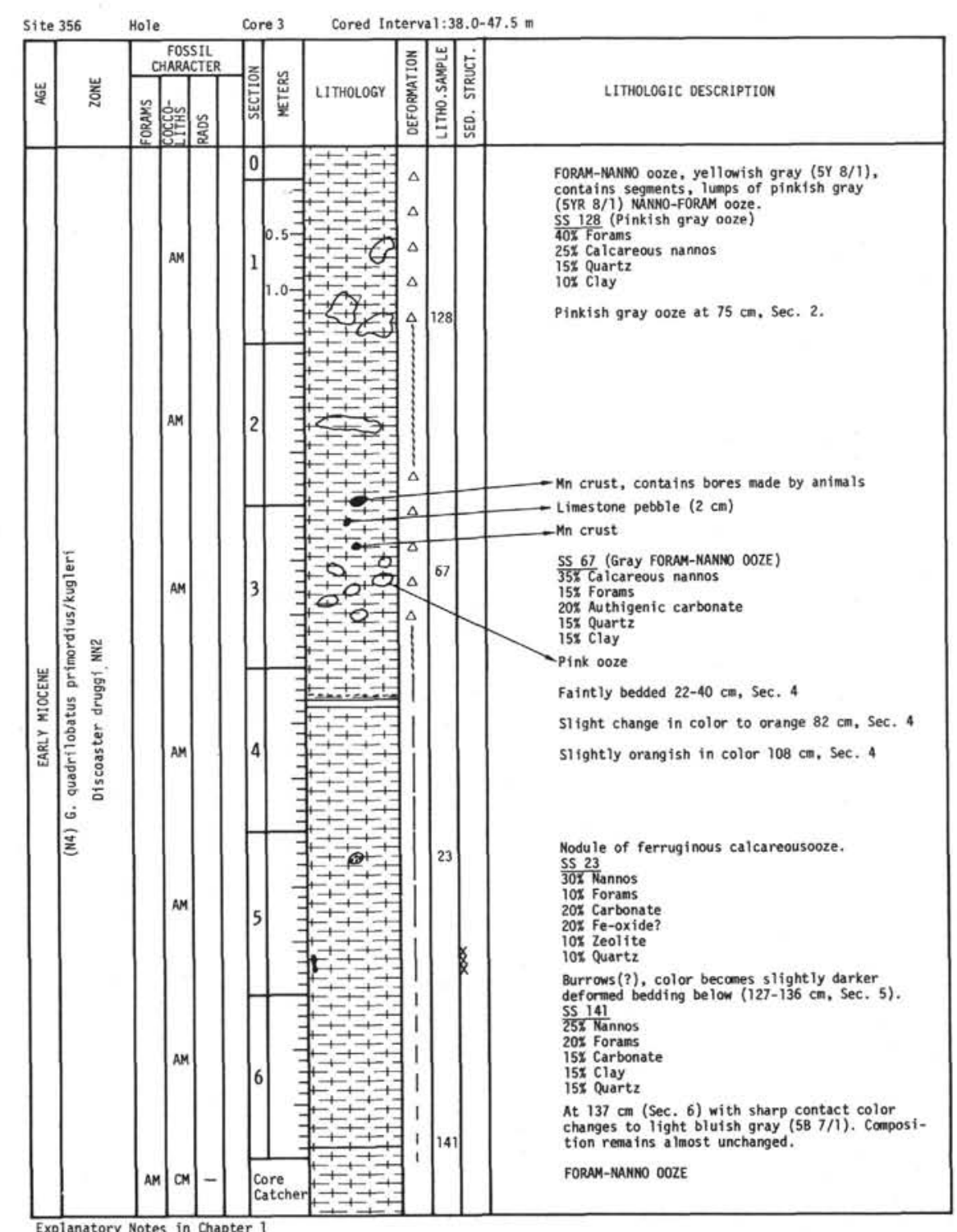




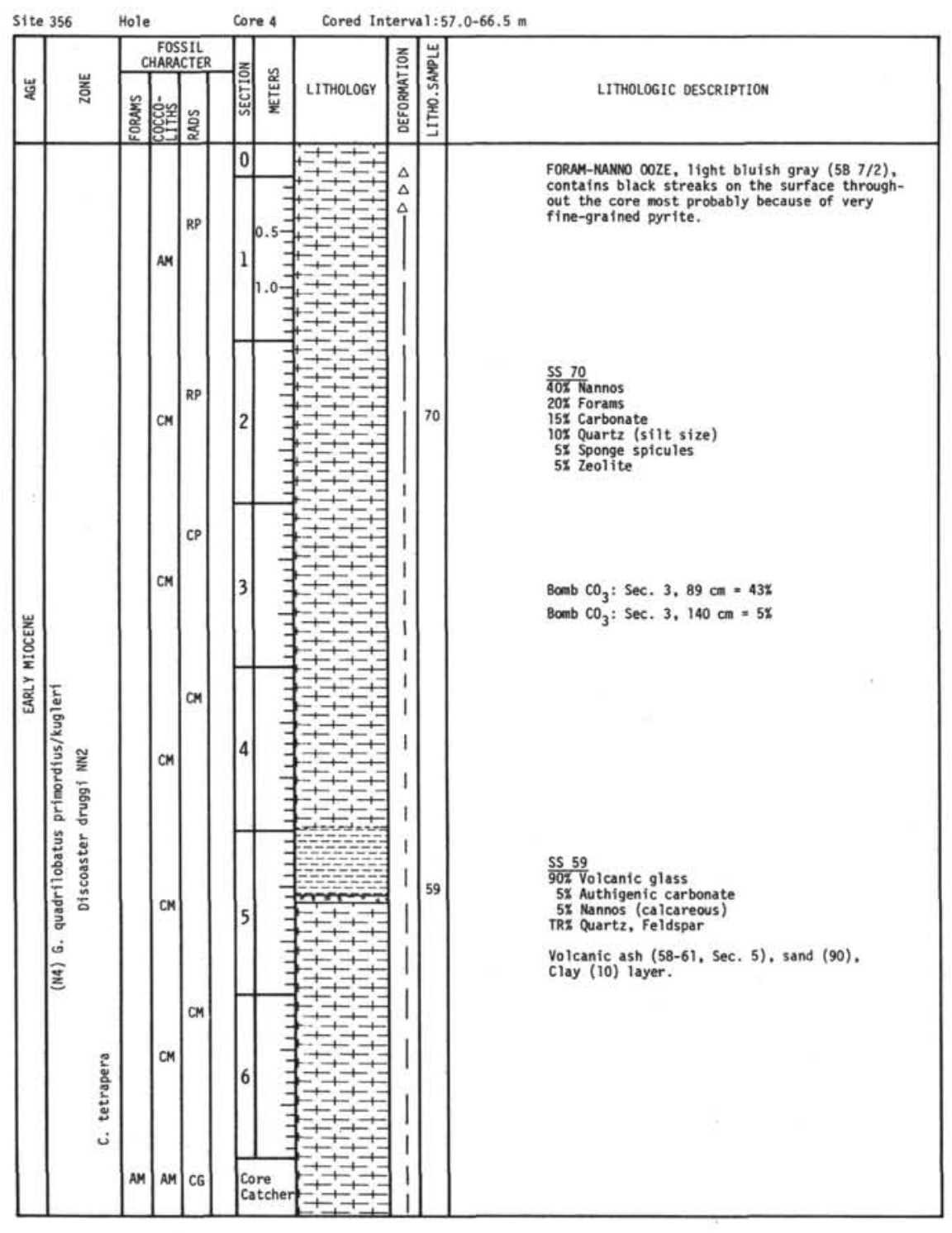

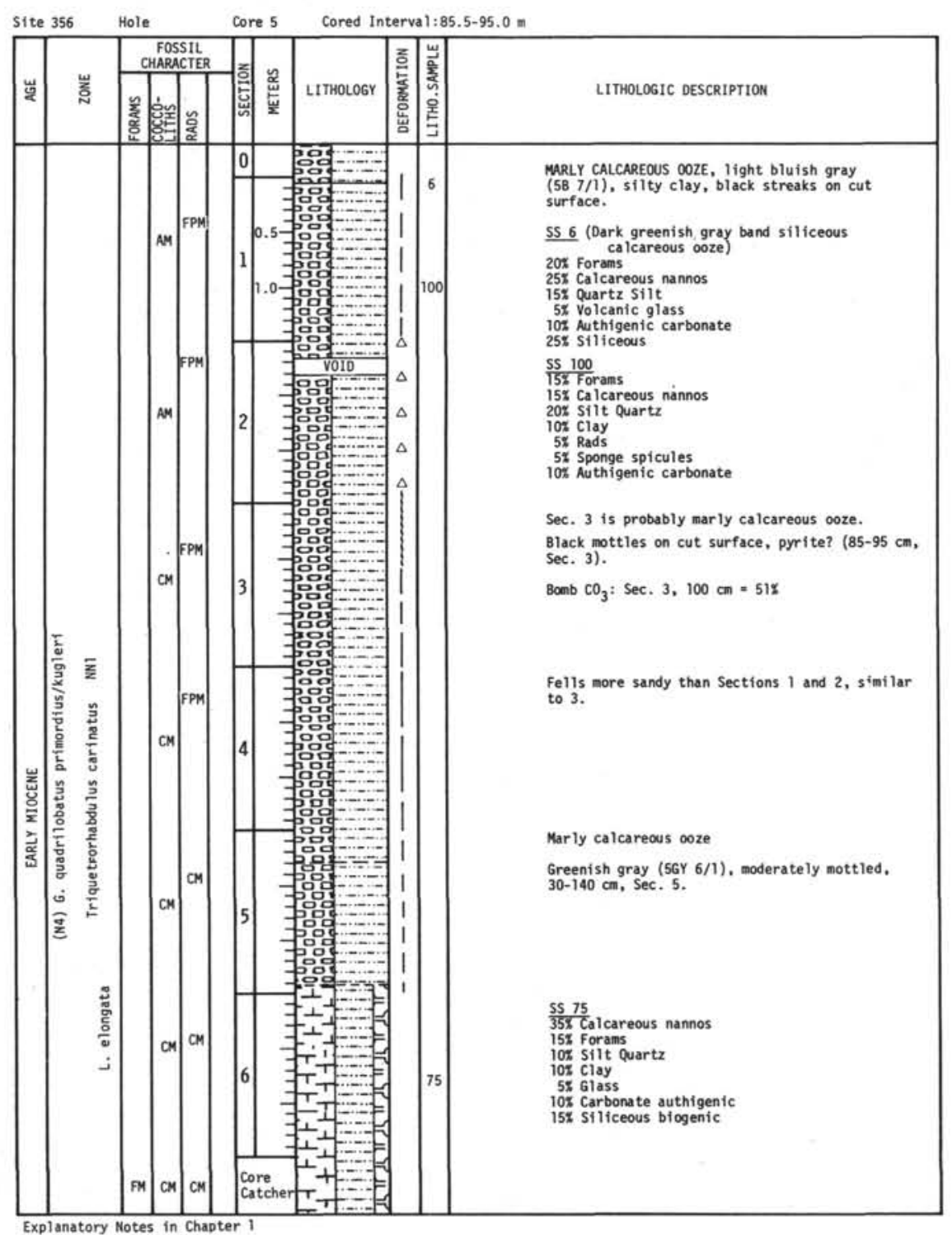




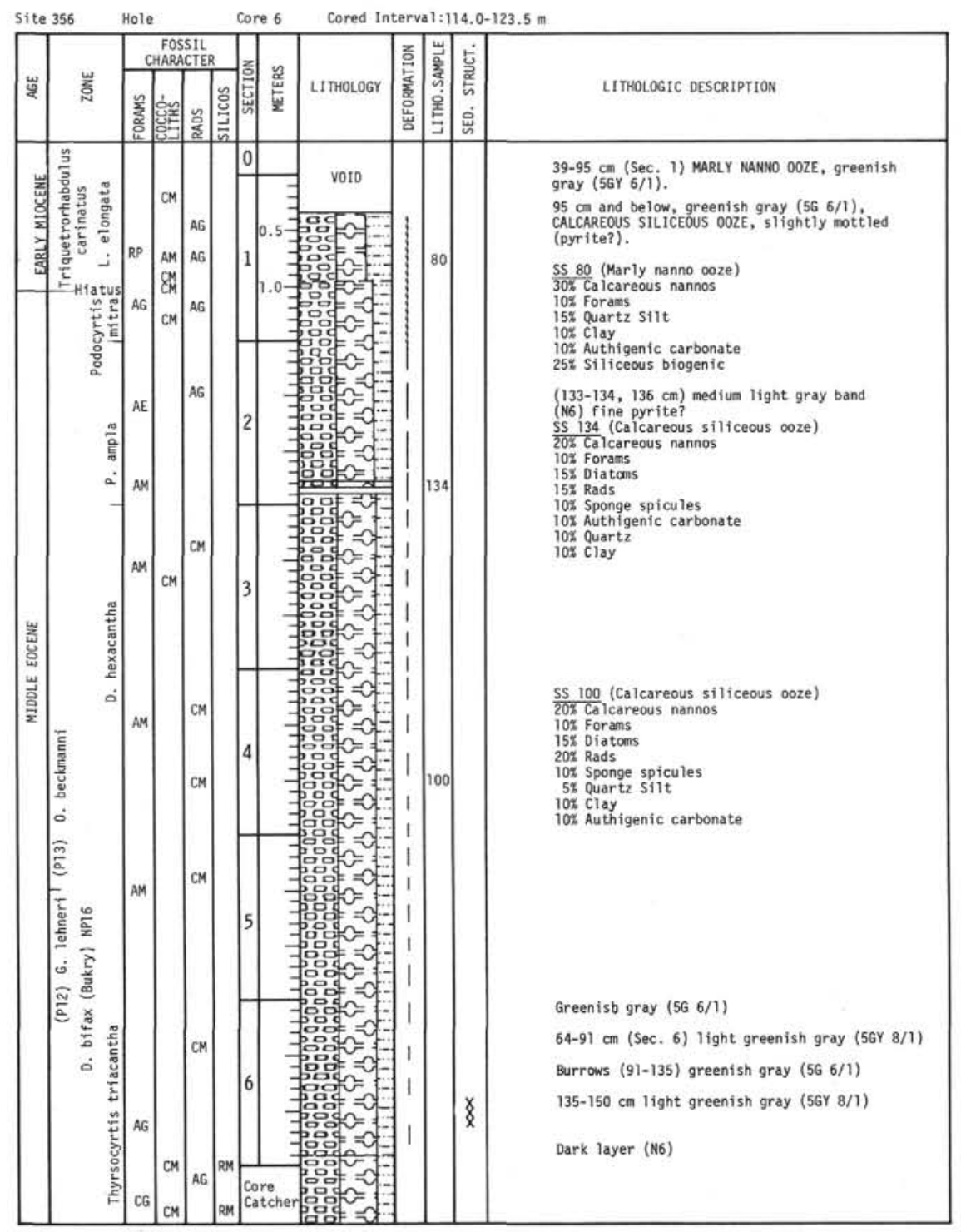

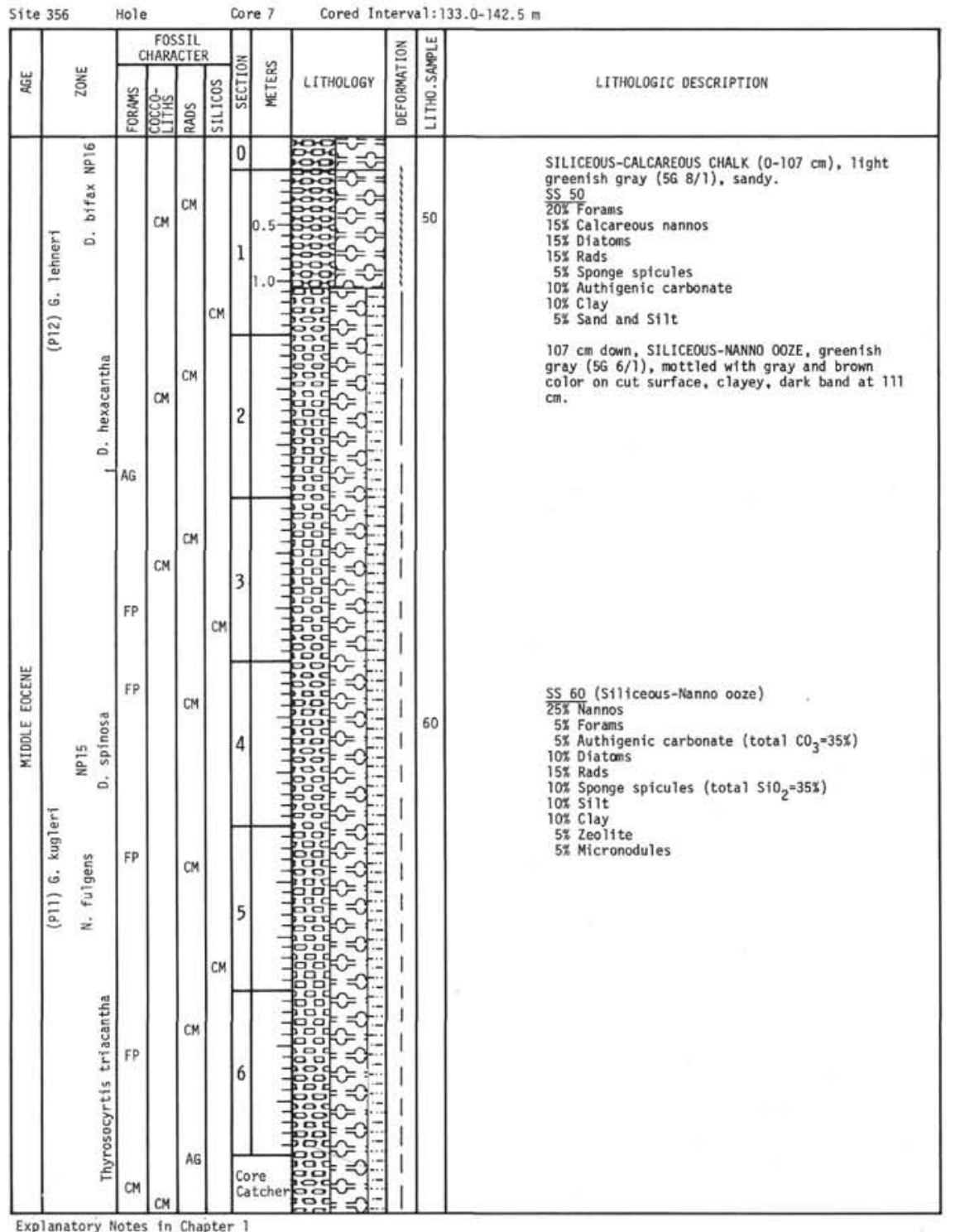




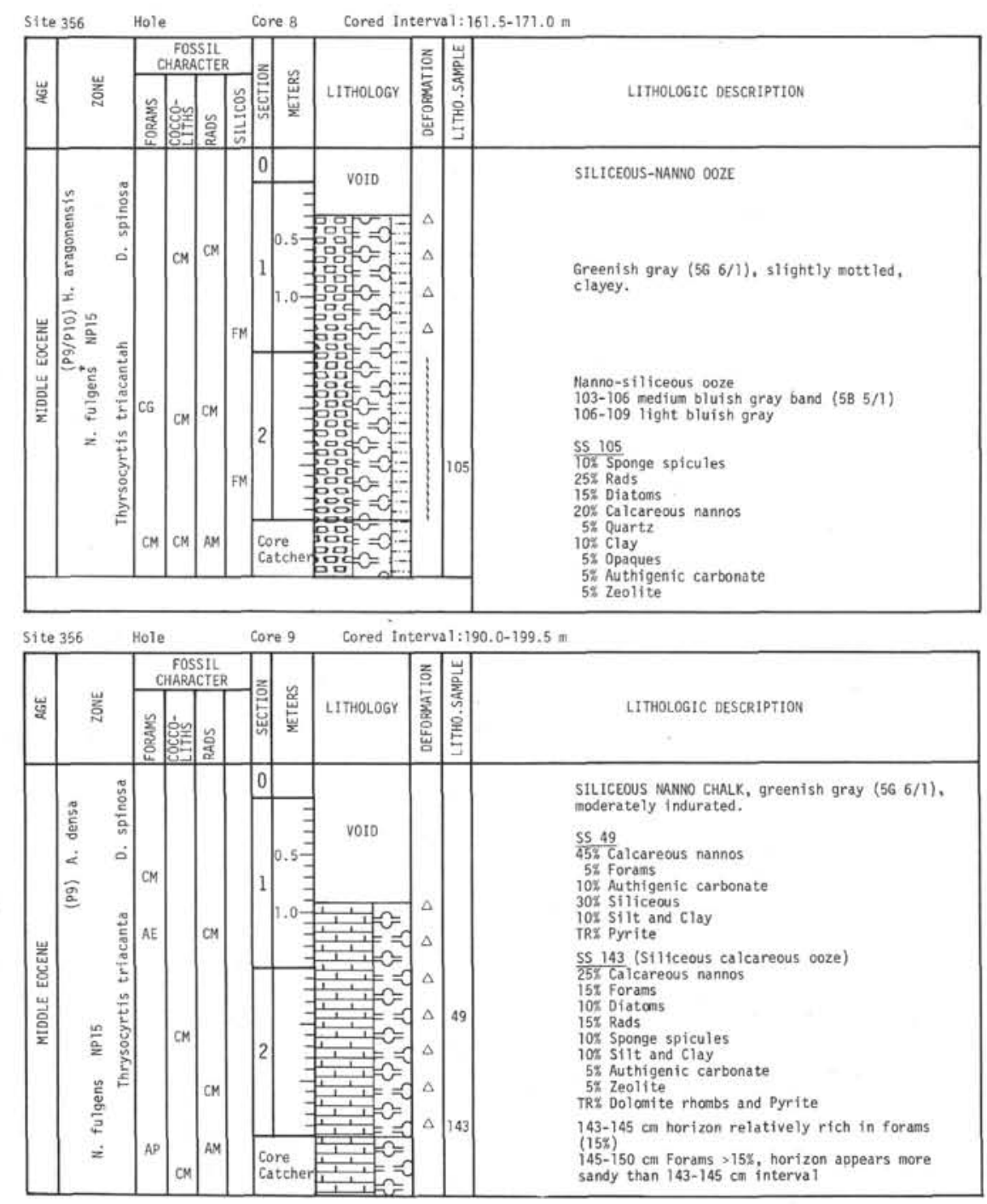

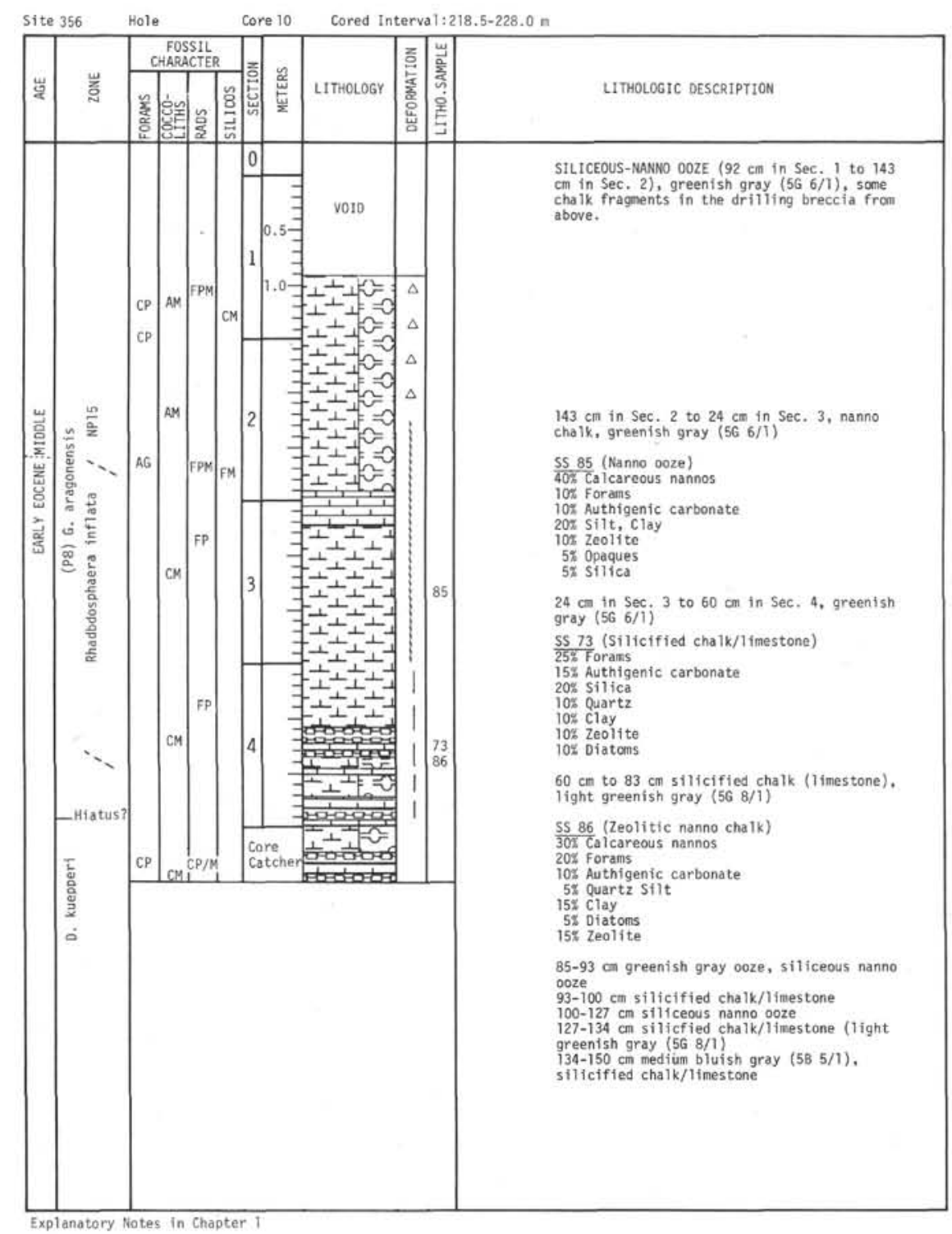




\begin{tabular}{|l|l|l|l|l|l|l|}
\hline & Site 356 \\
\hline
\end{tabular}

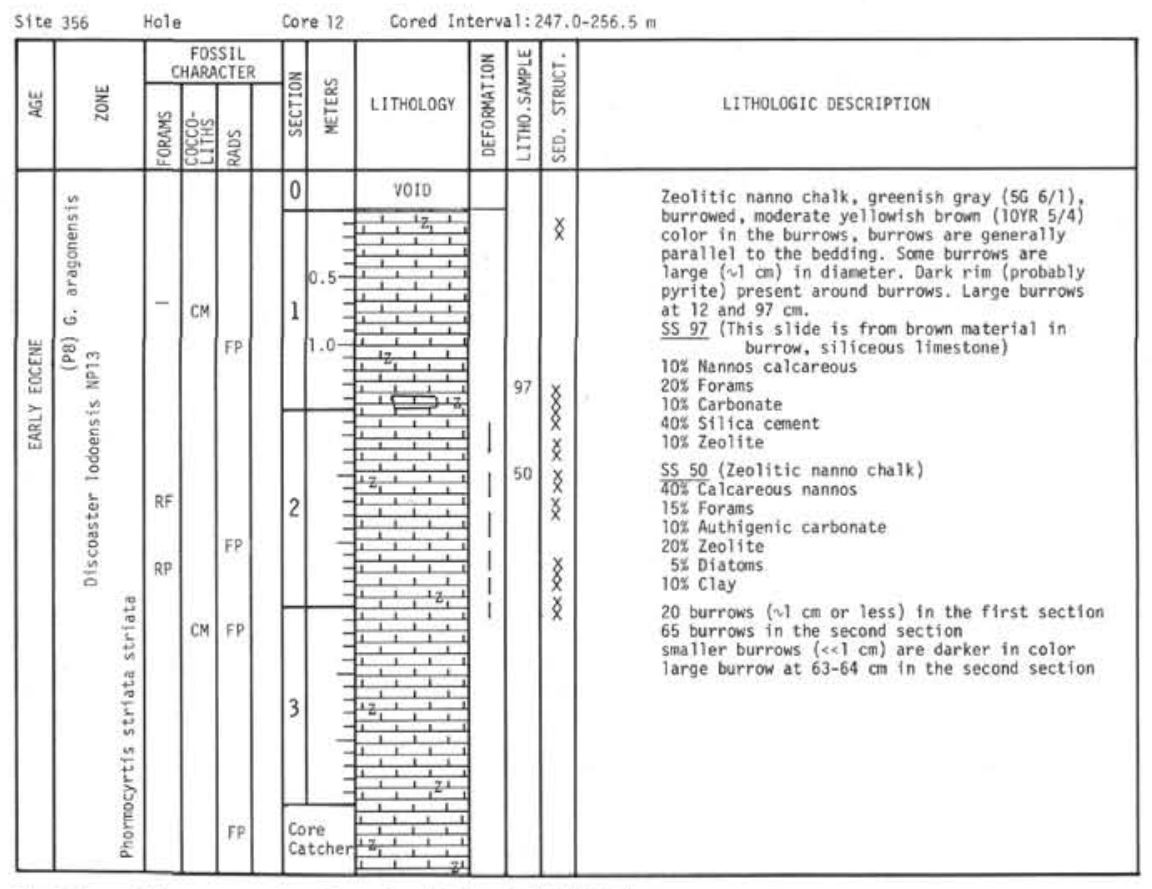

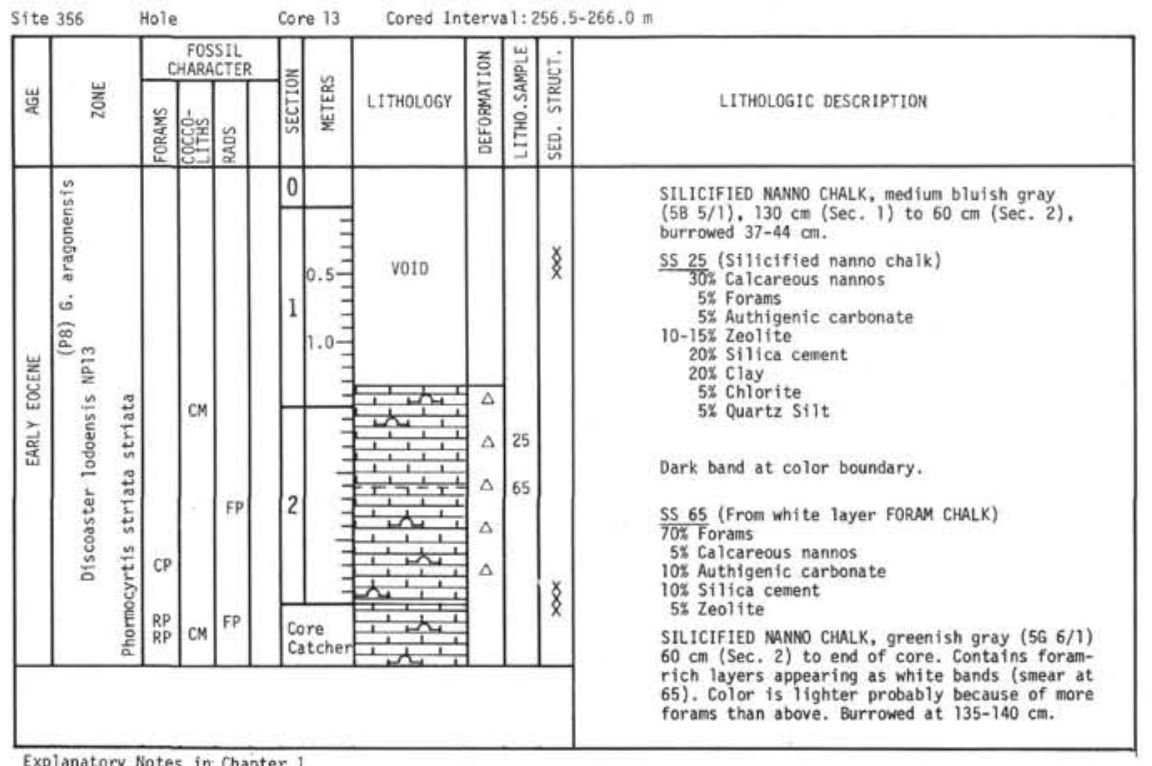




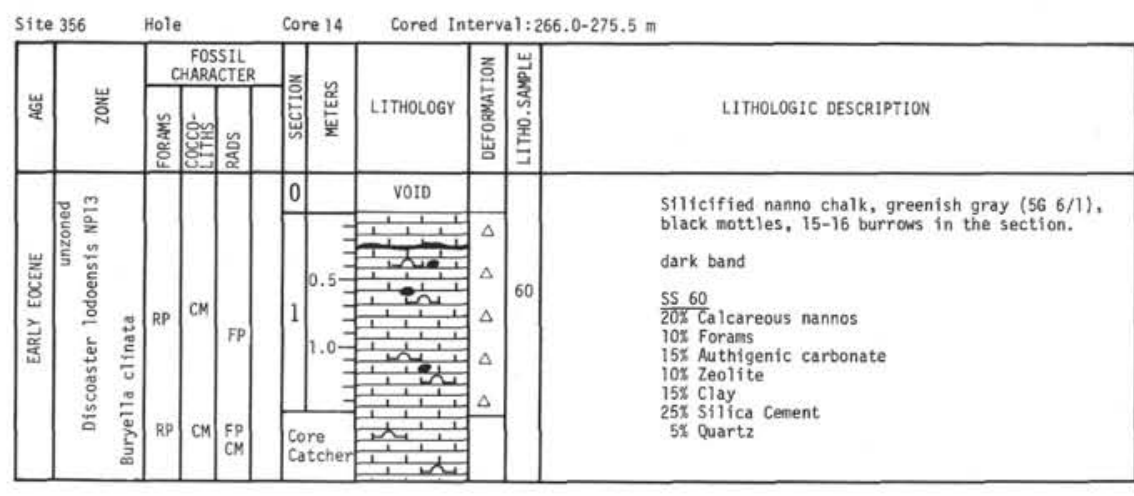

S1te 356 Hole

Core 15 Cored Interva 1:275.5-285.0

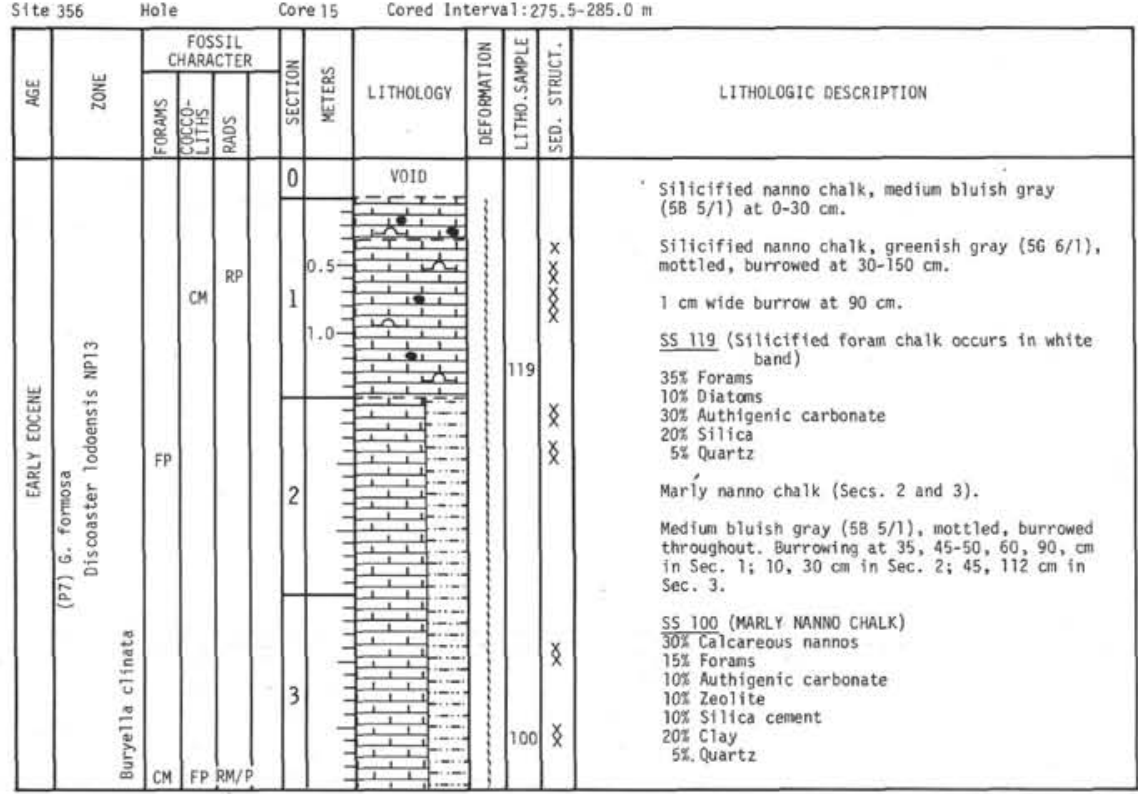

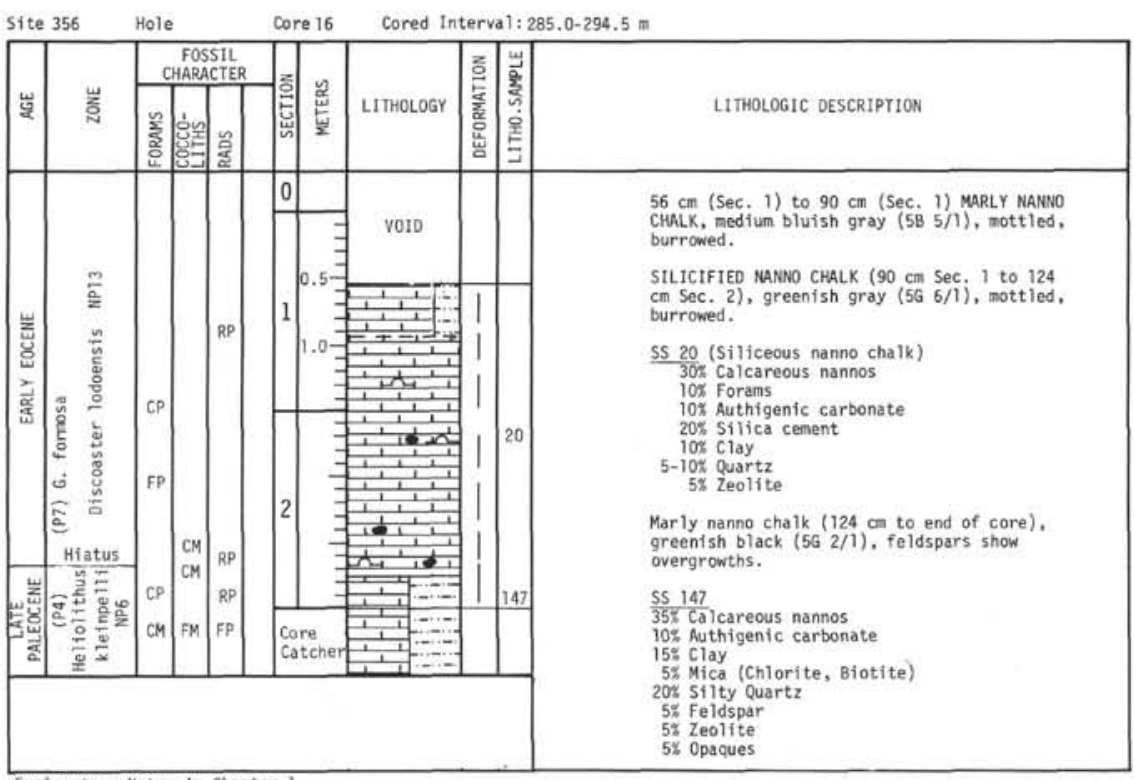

Explanatory Notes in Chapter 1 


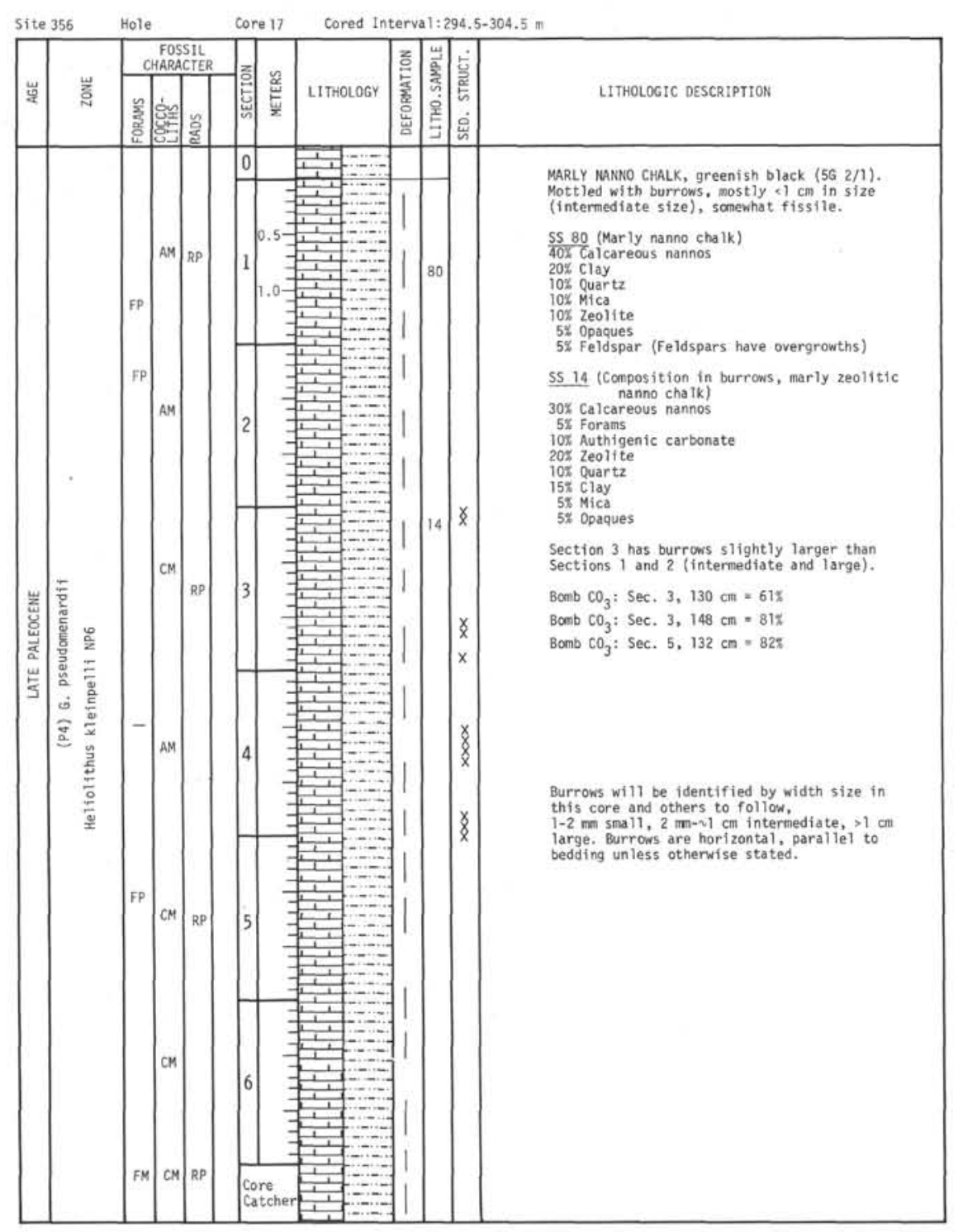

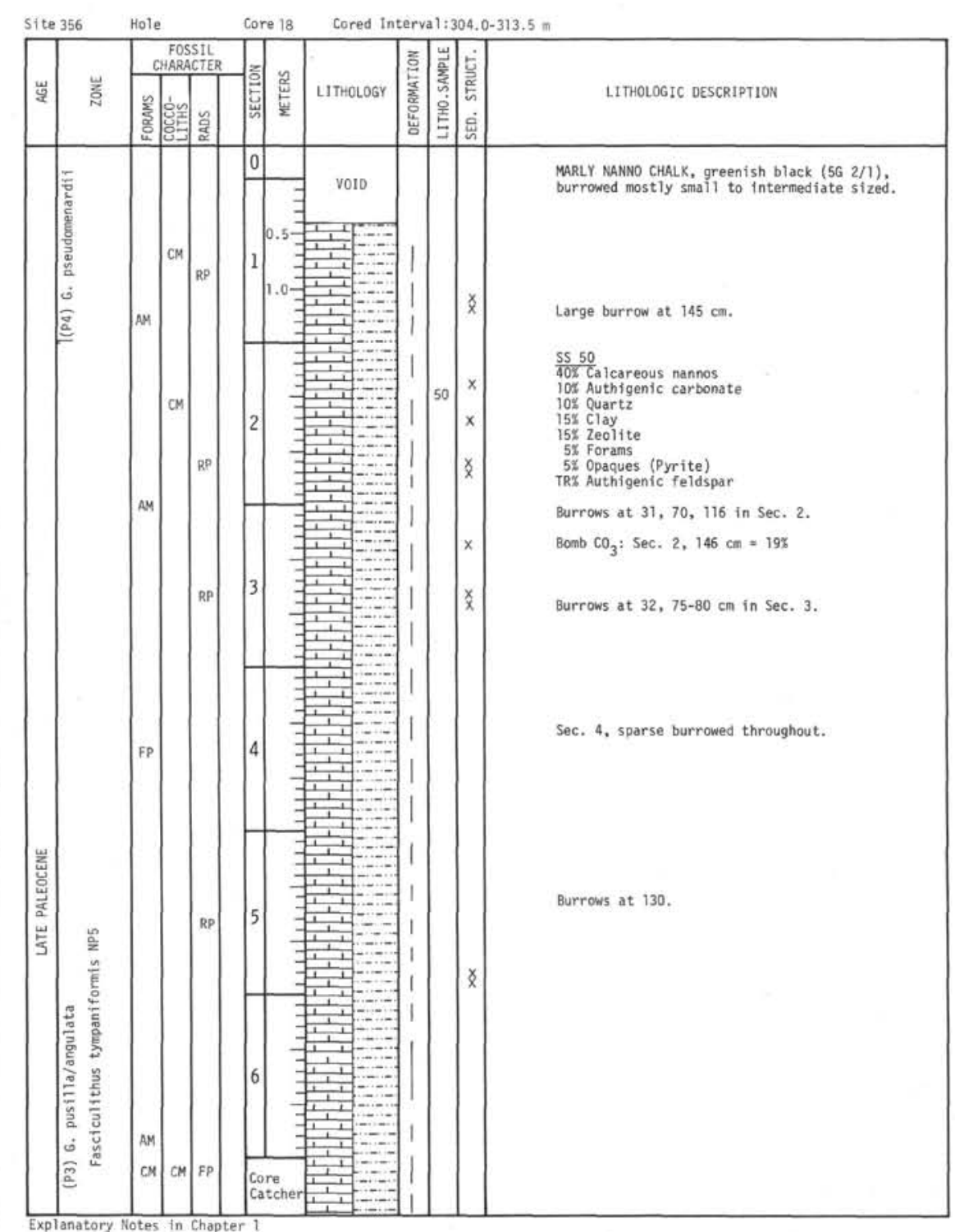



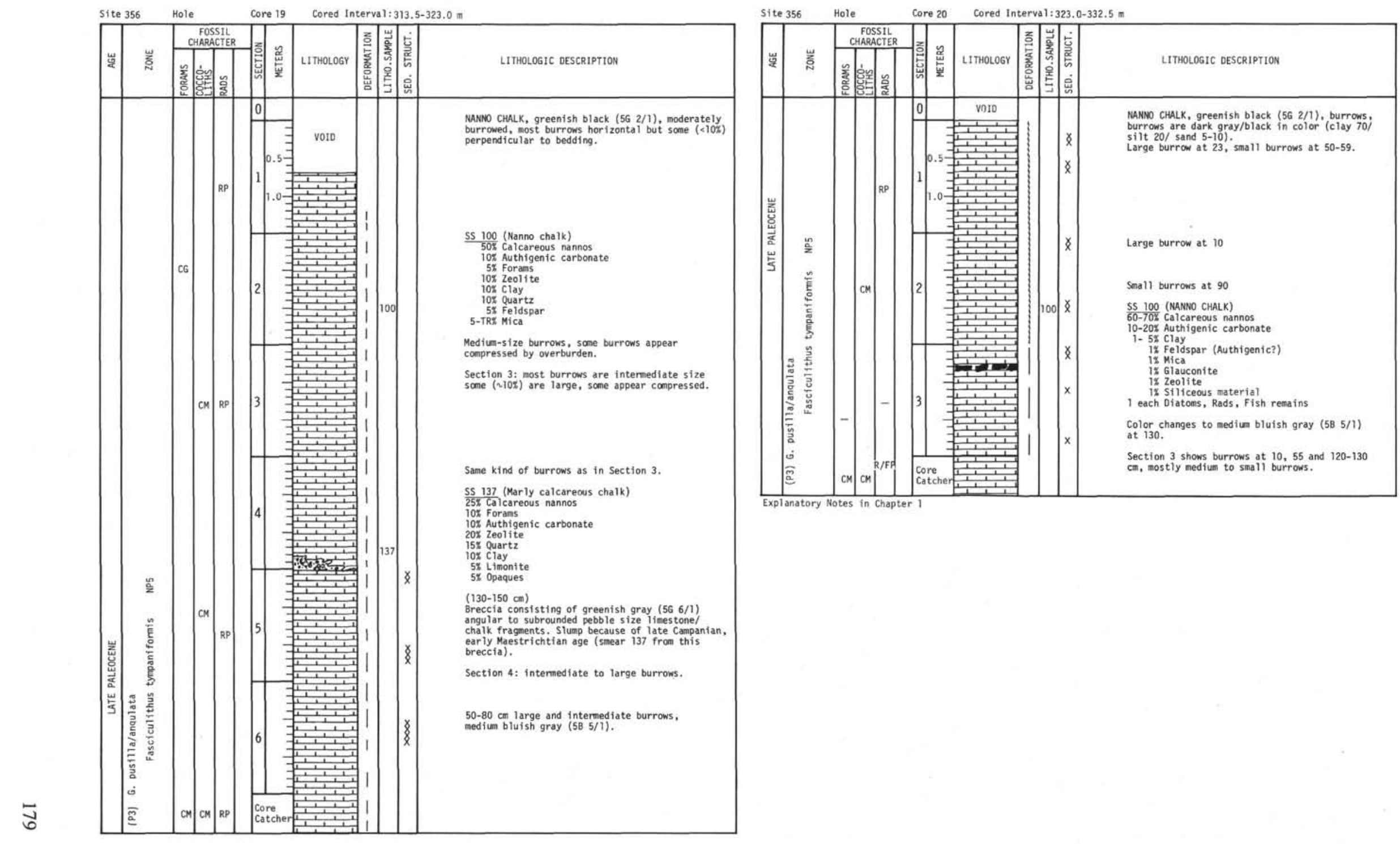

Explanatory Notes in Chapter- 


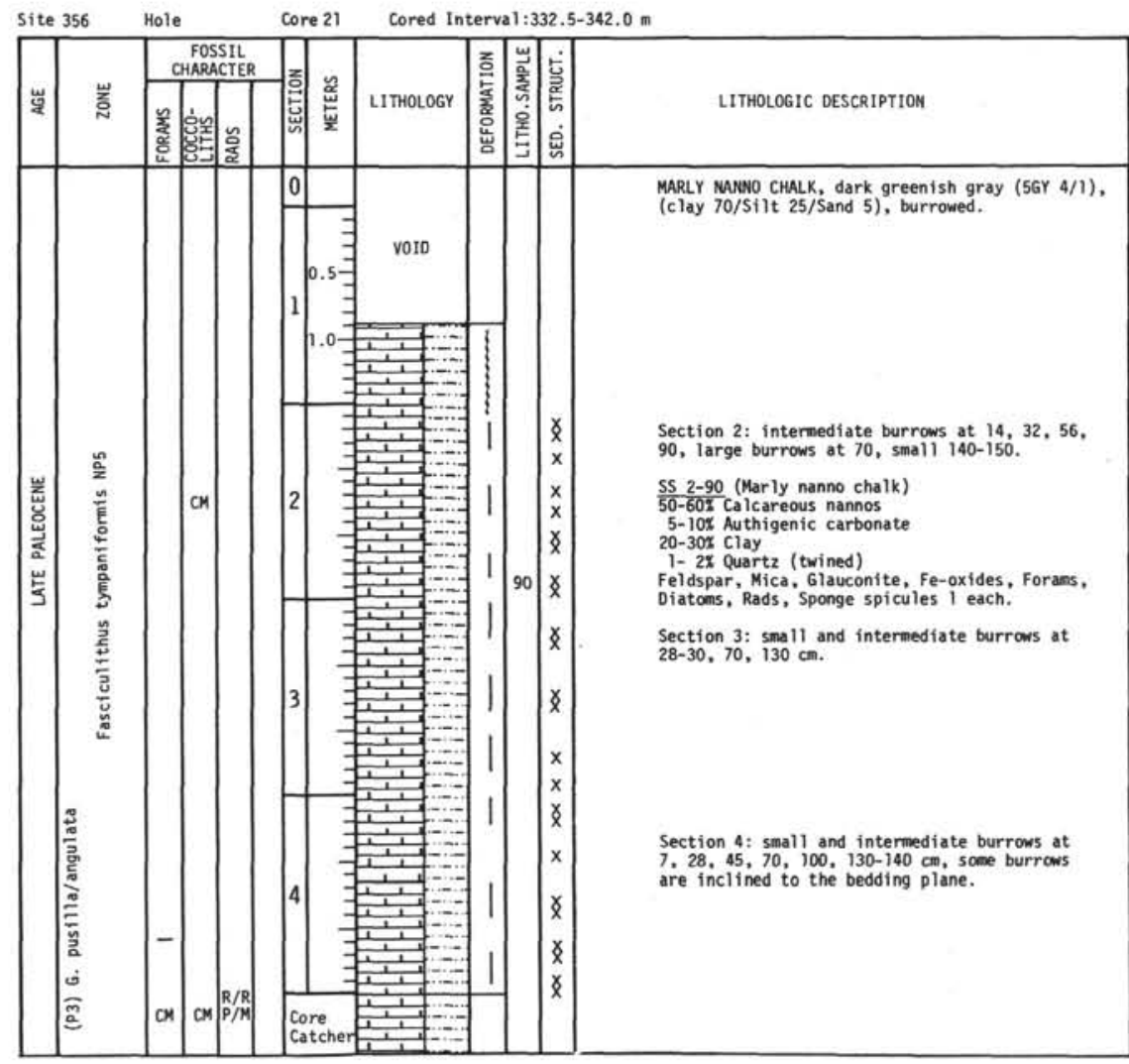

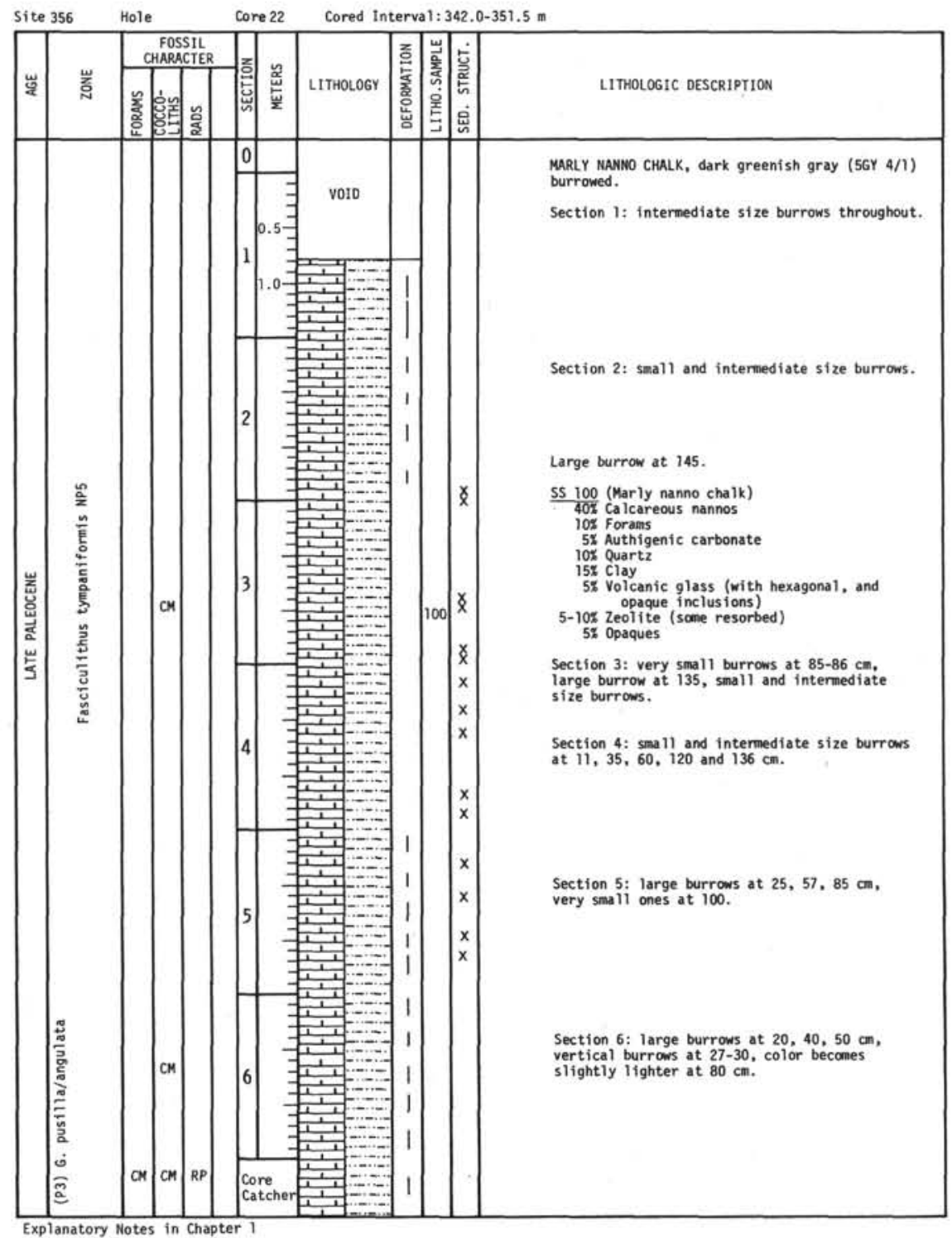



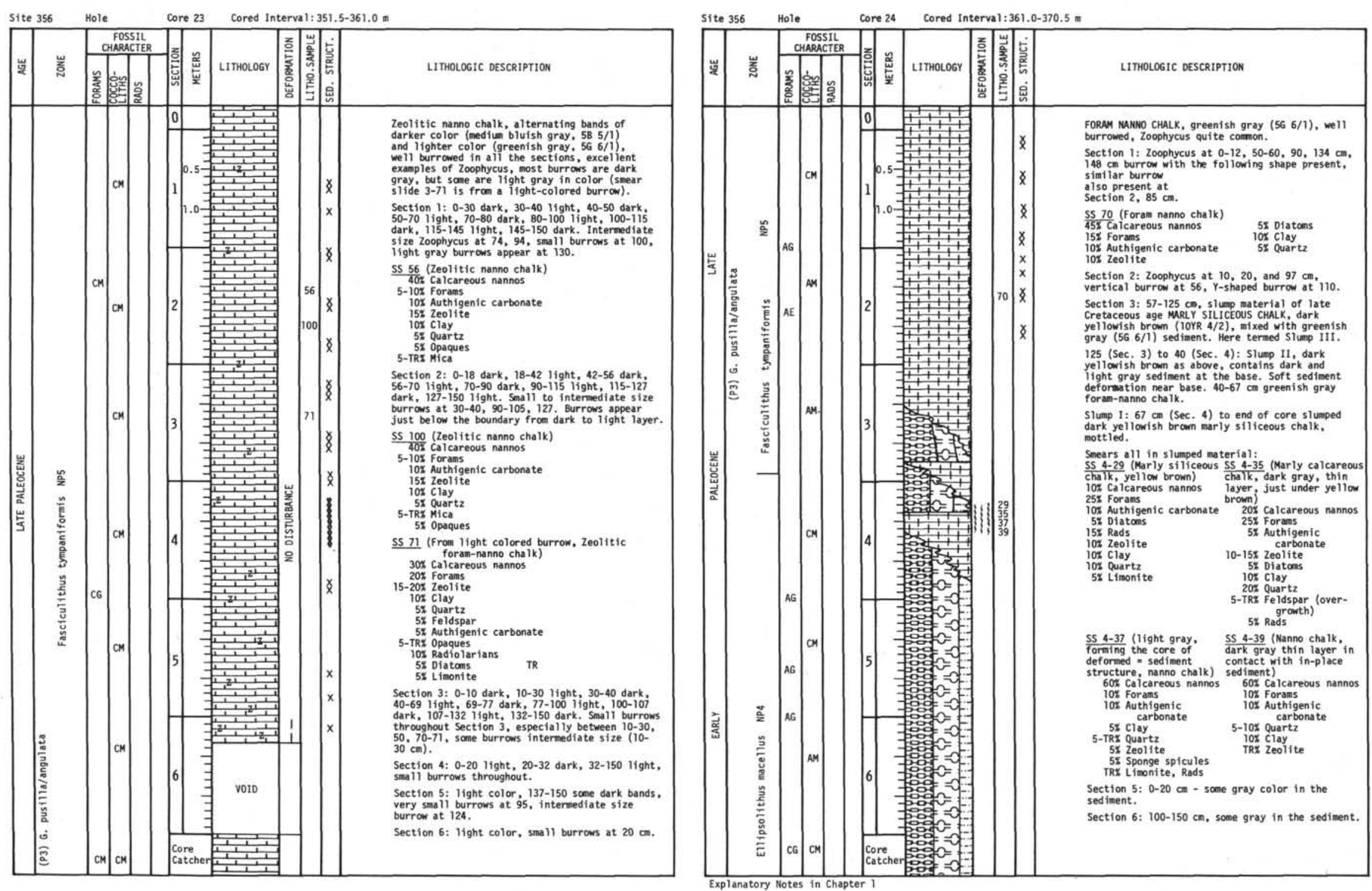

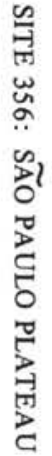




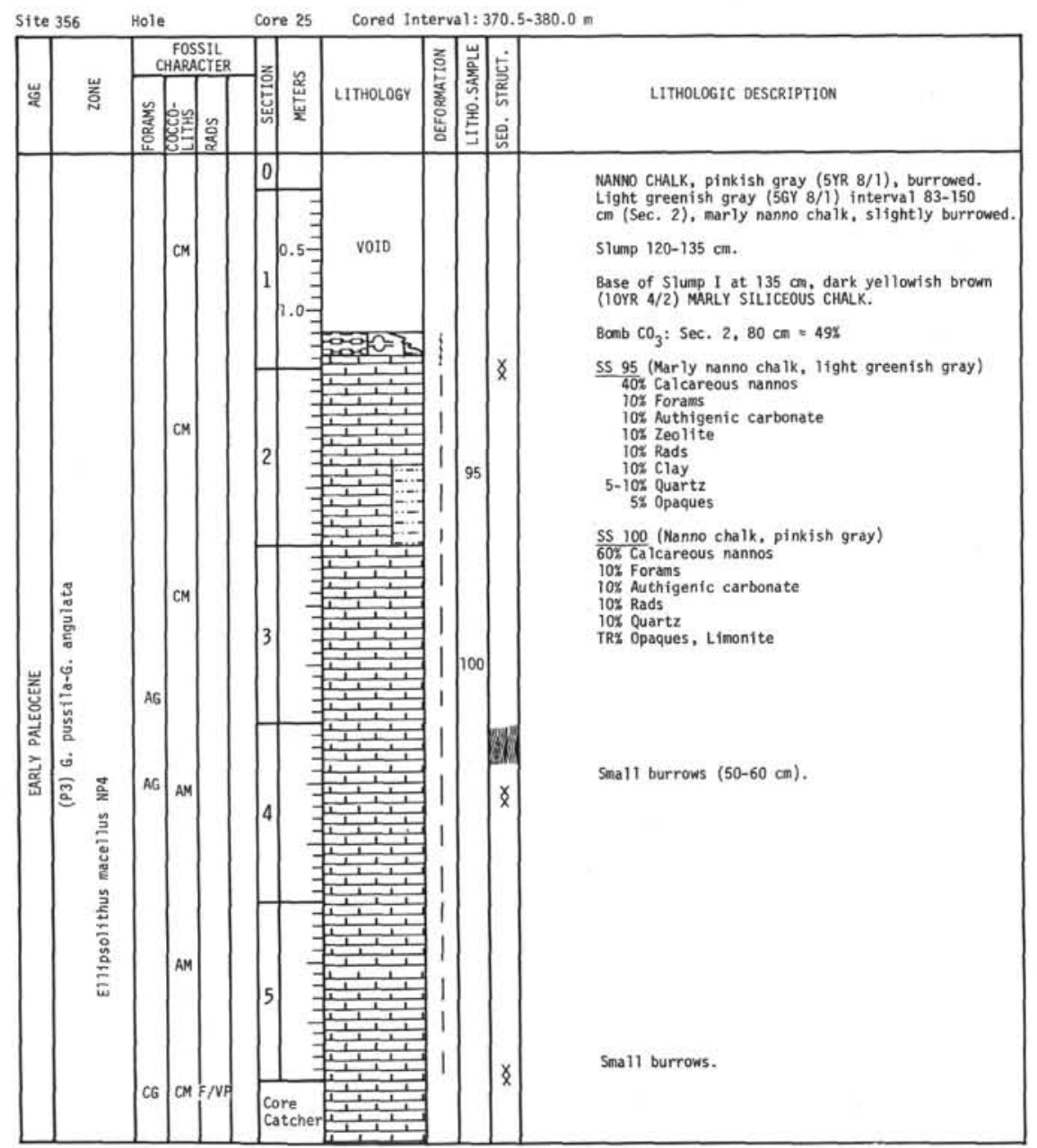

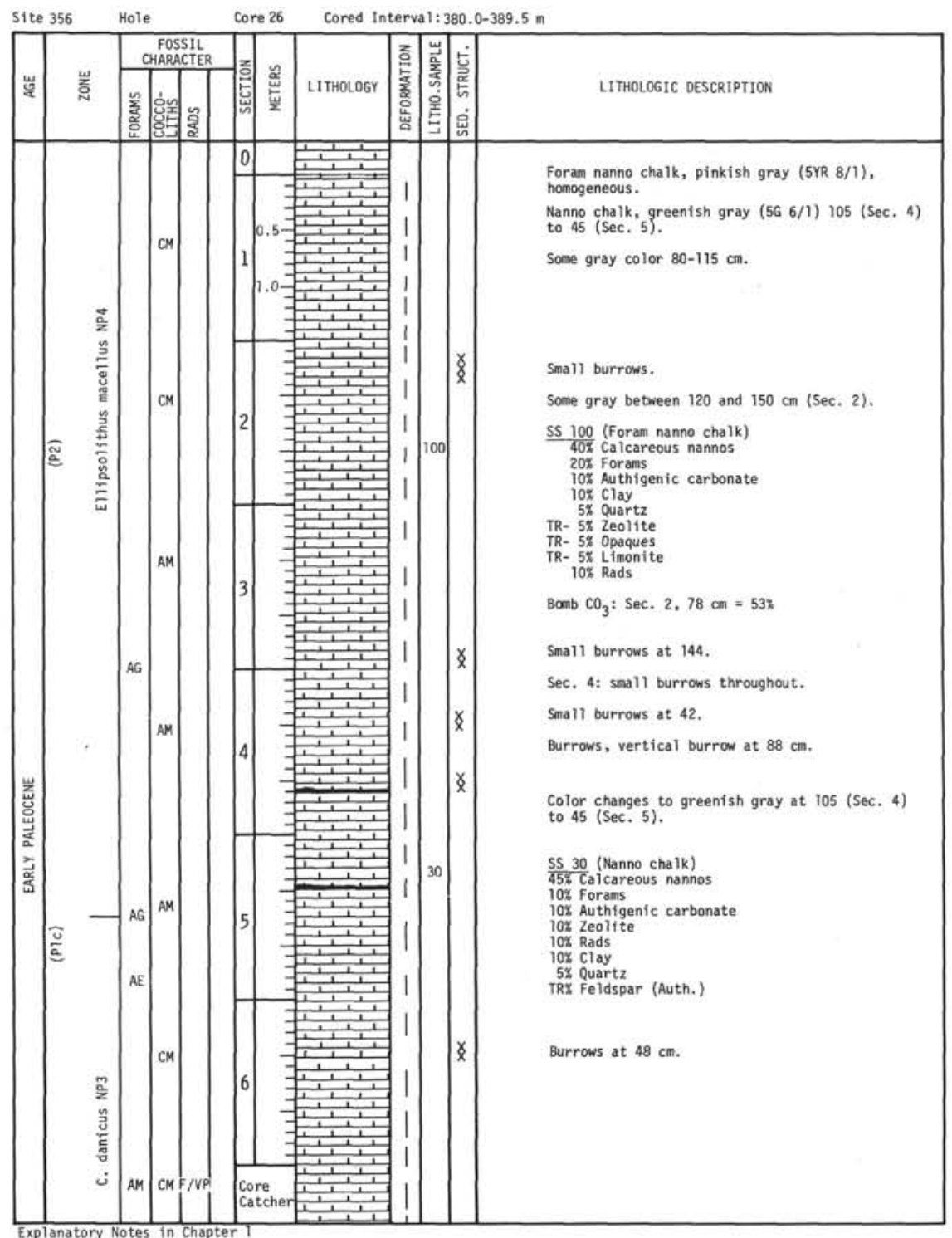






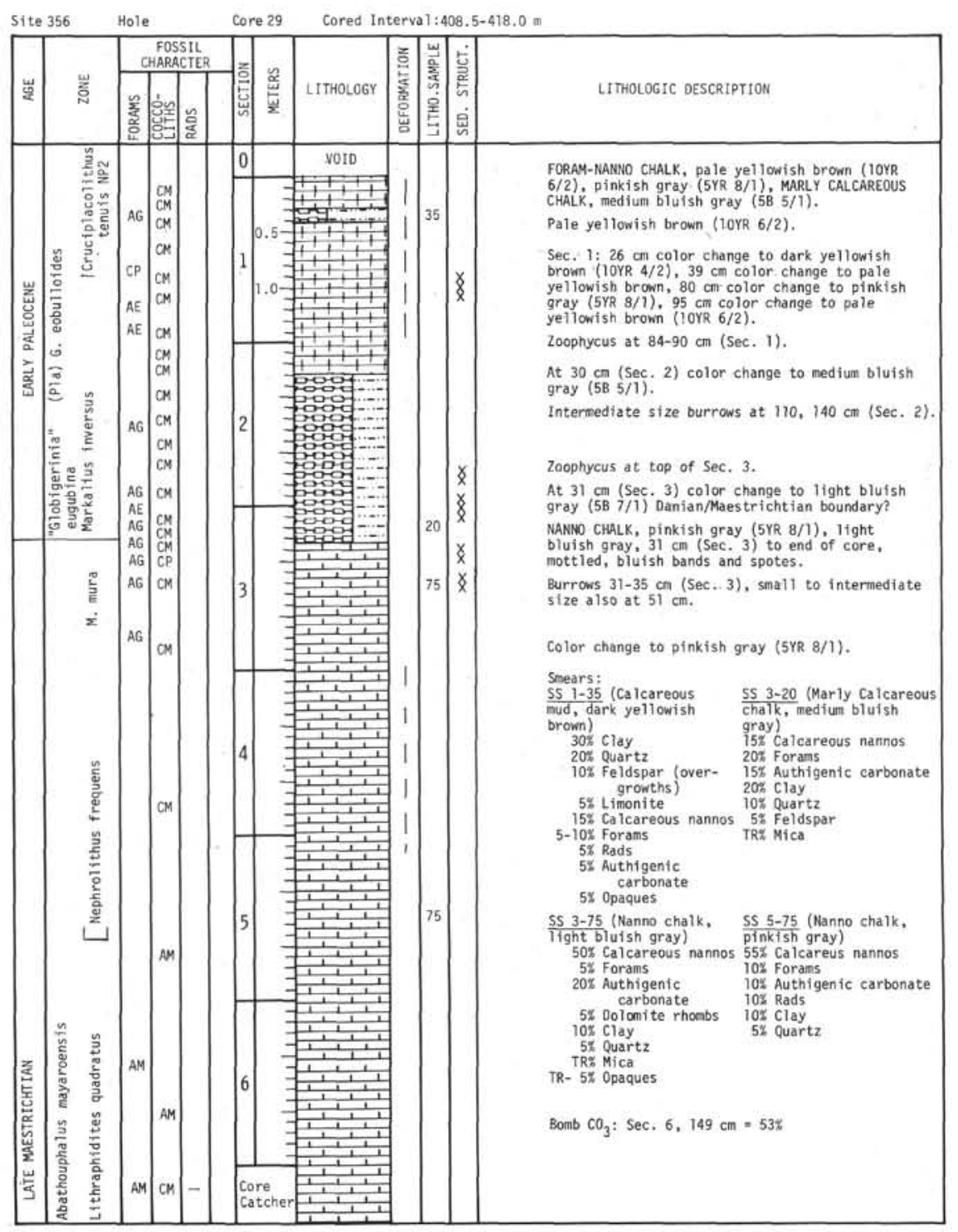

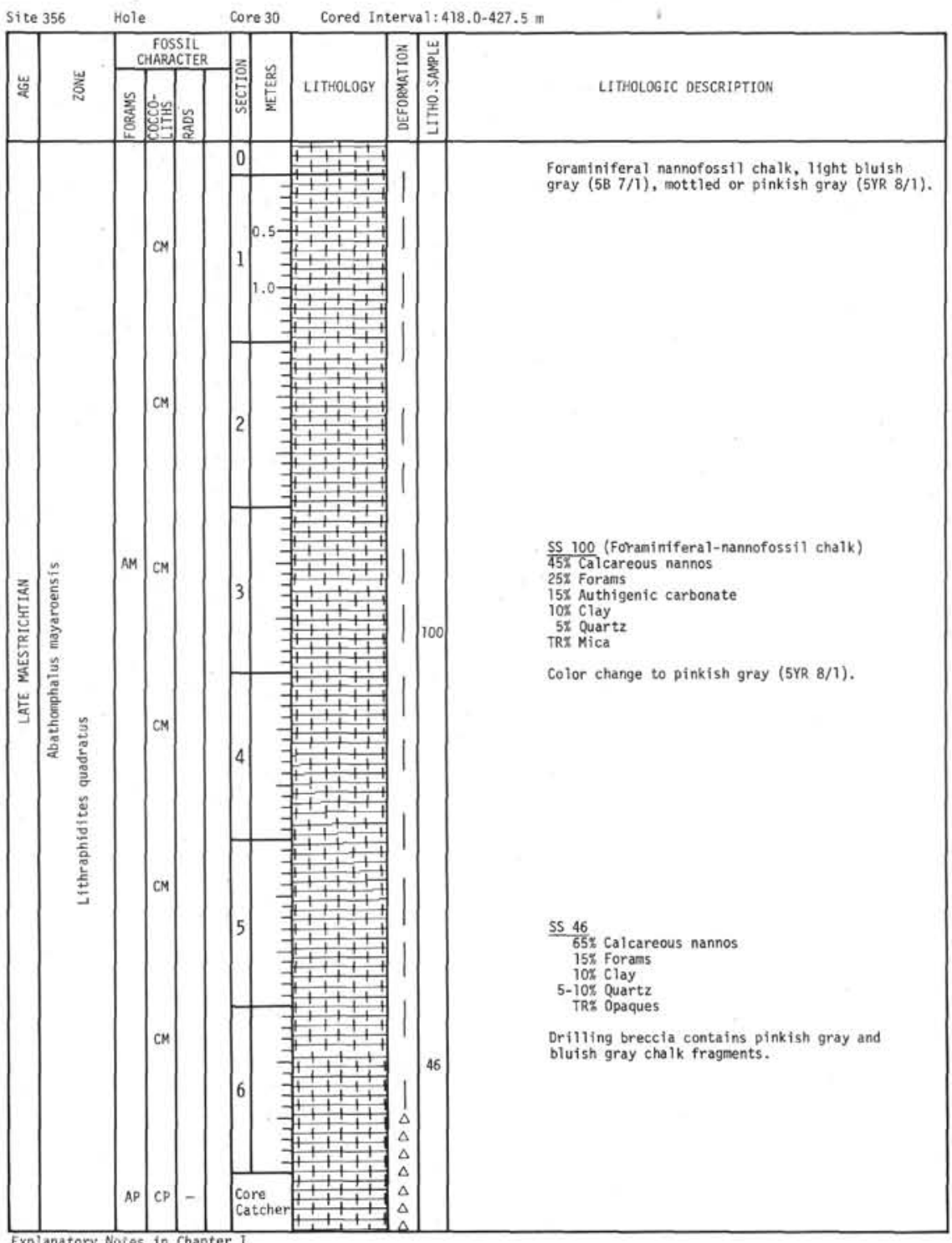



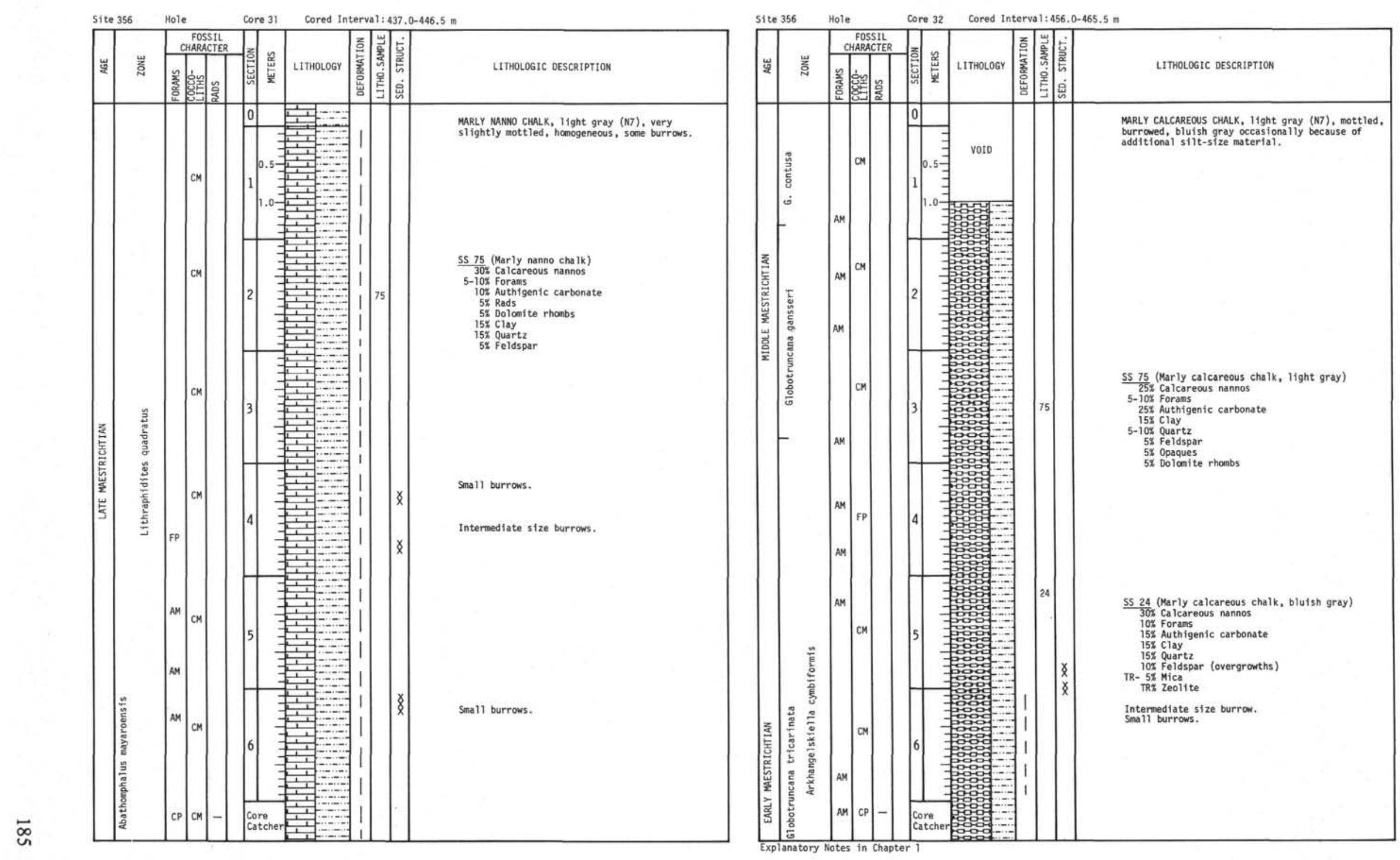


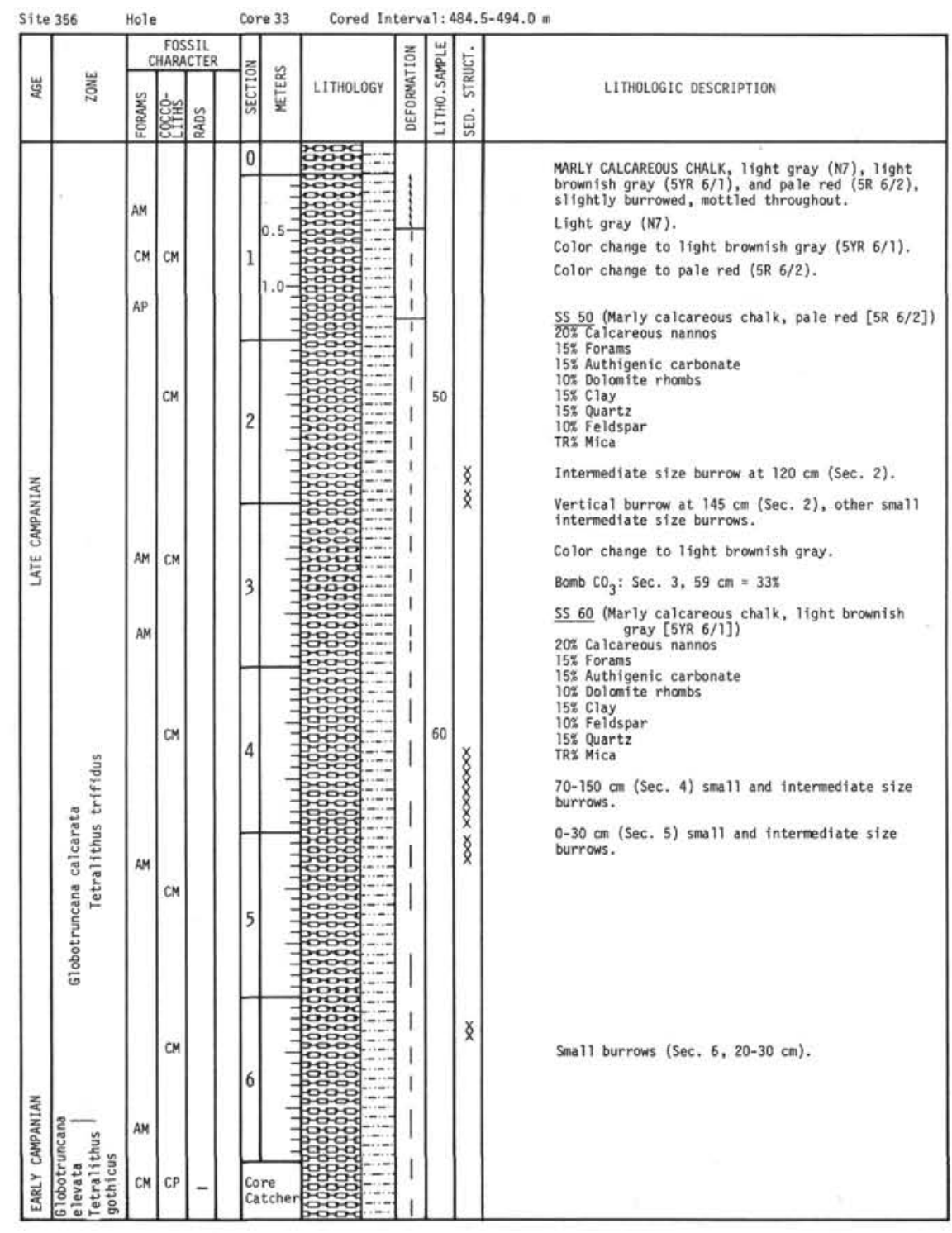

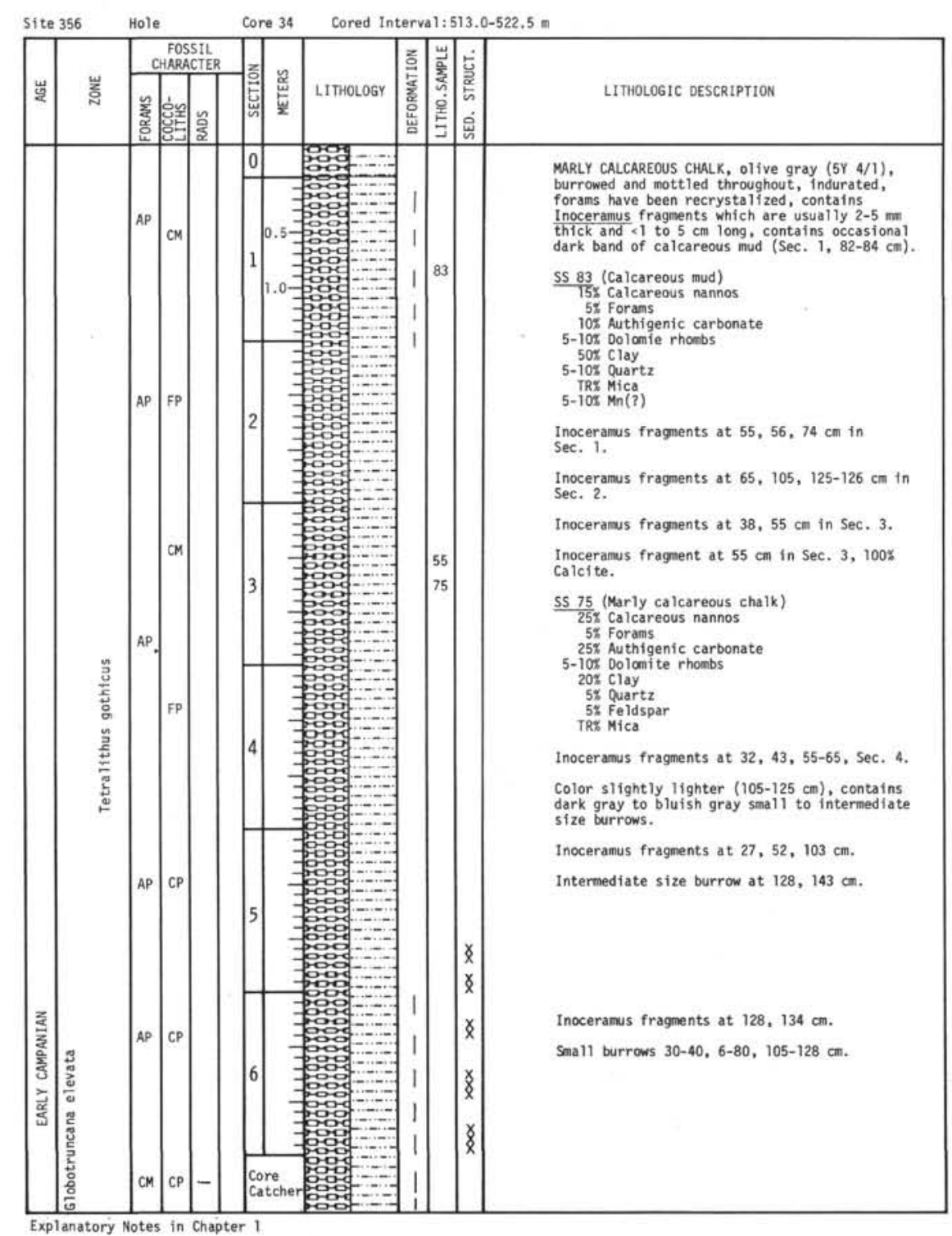



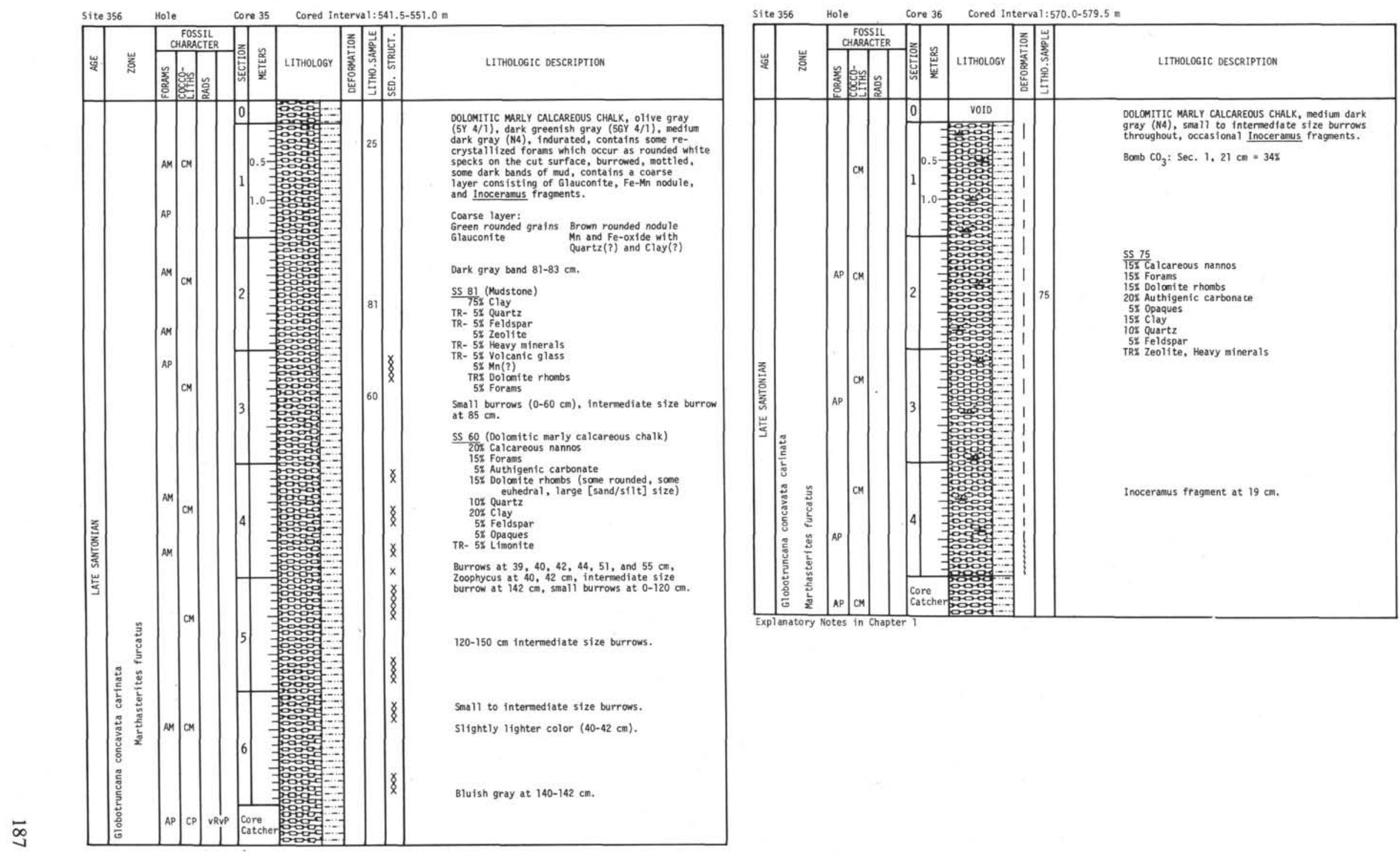


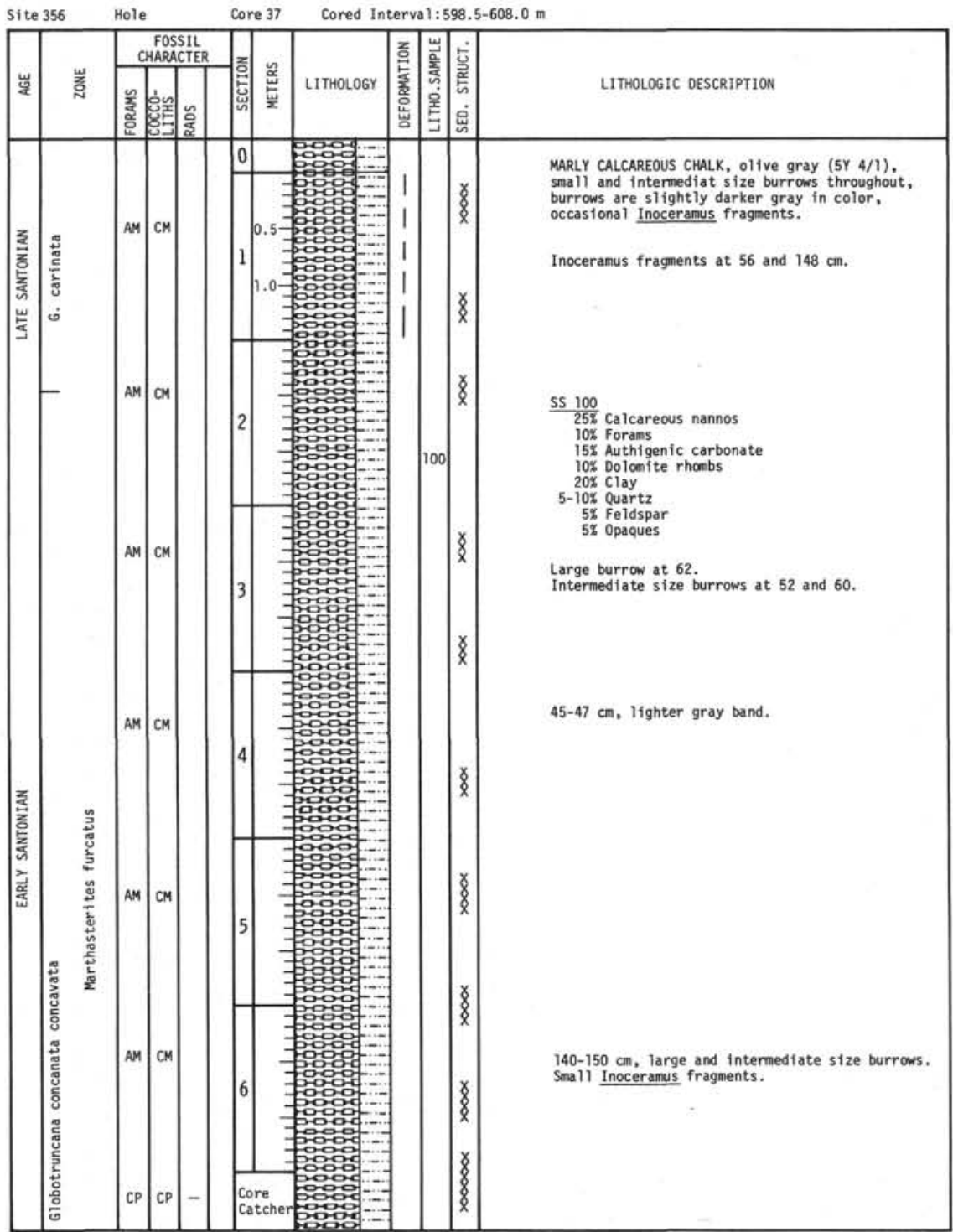

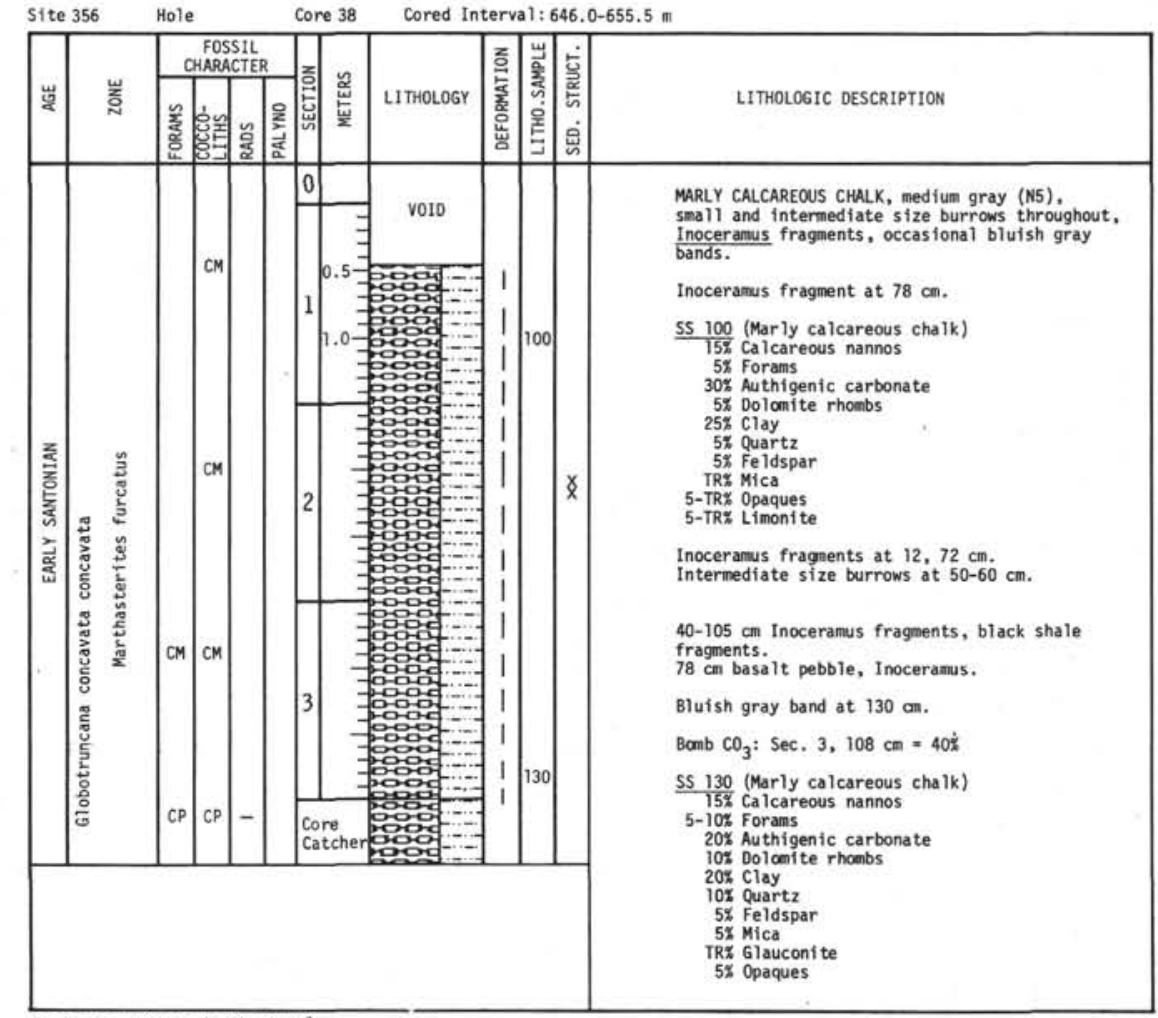

Explanatory Notes in Chapter 1 

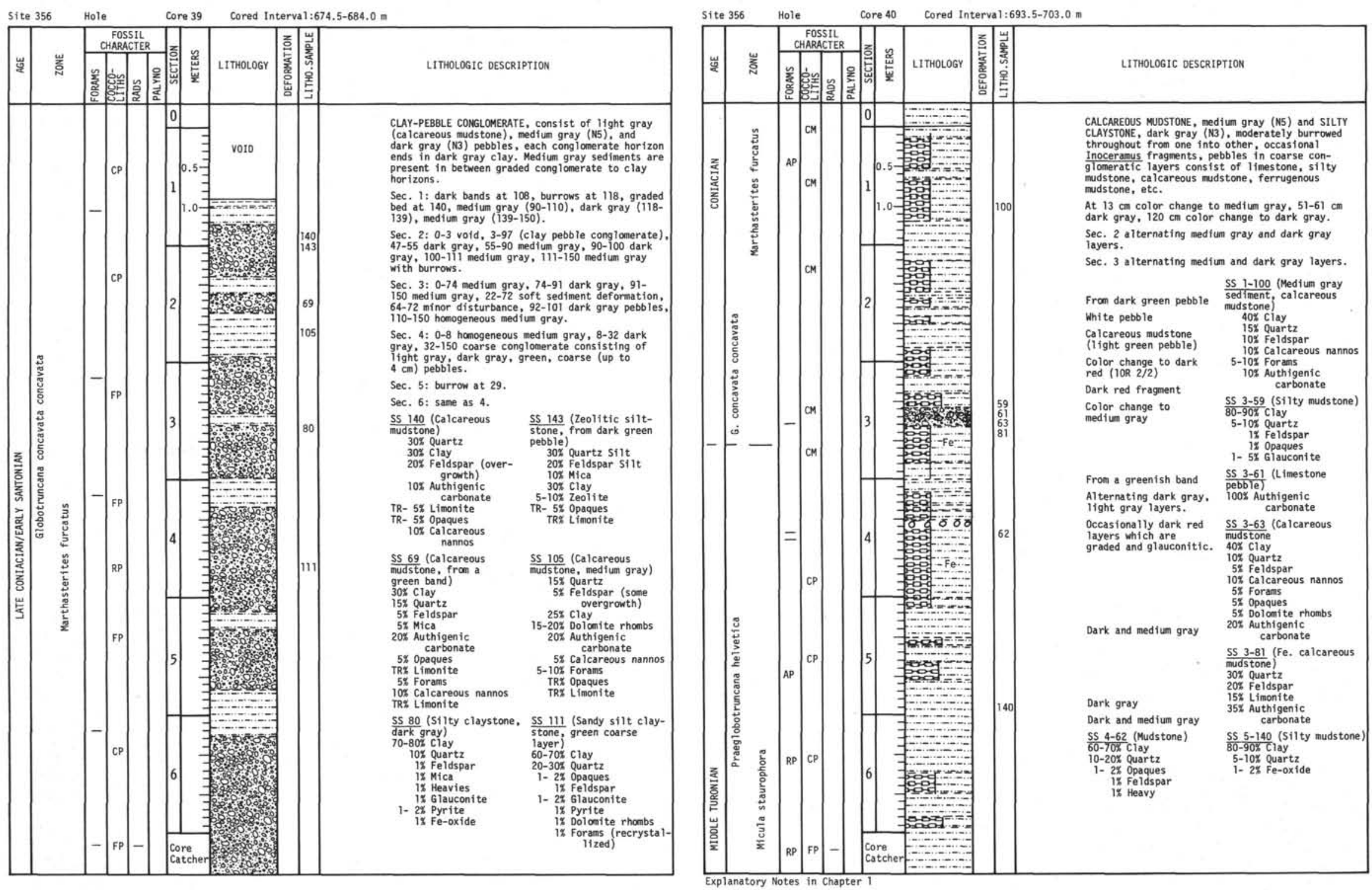


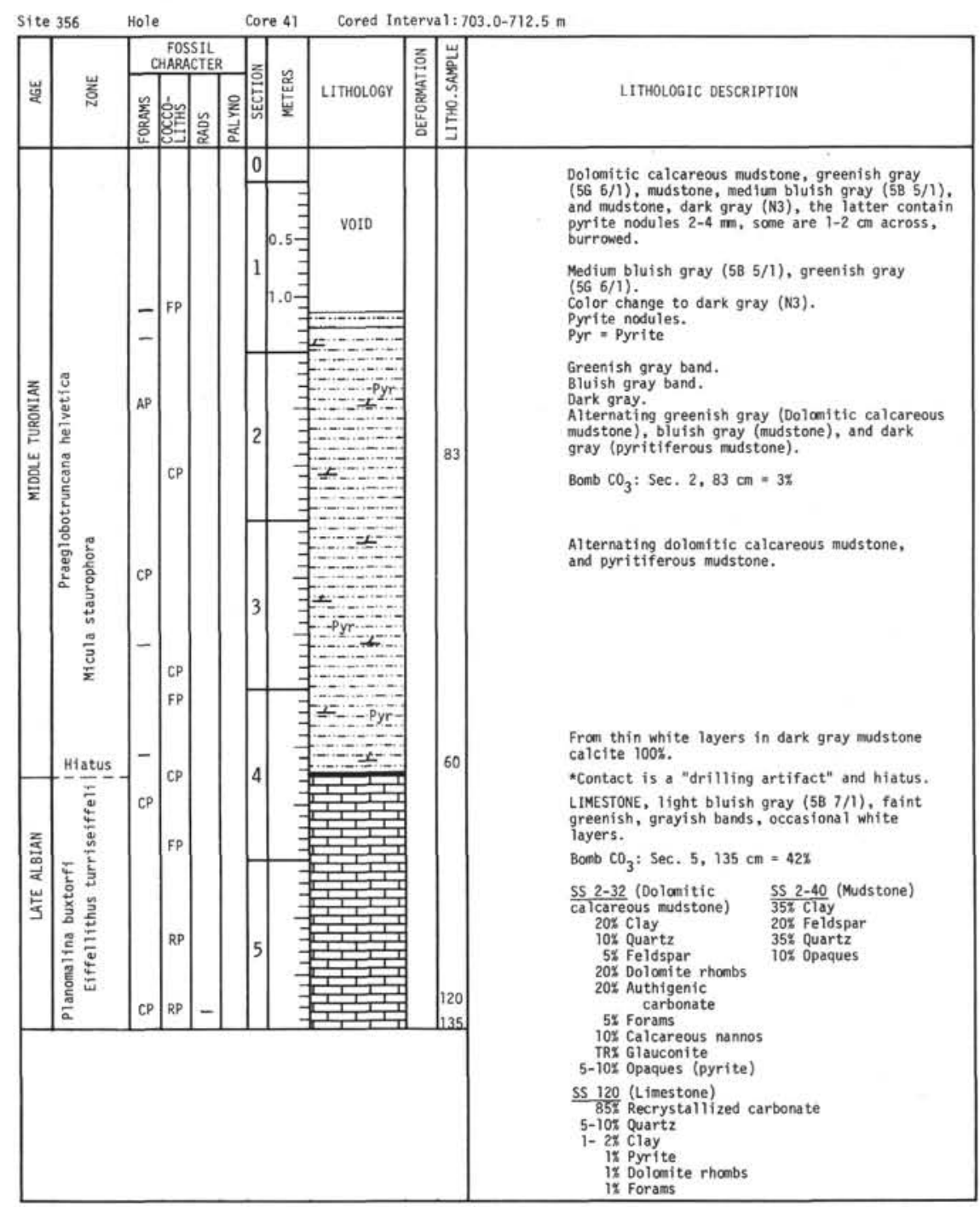

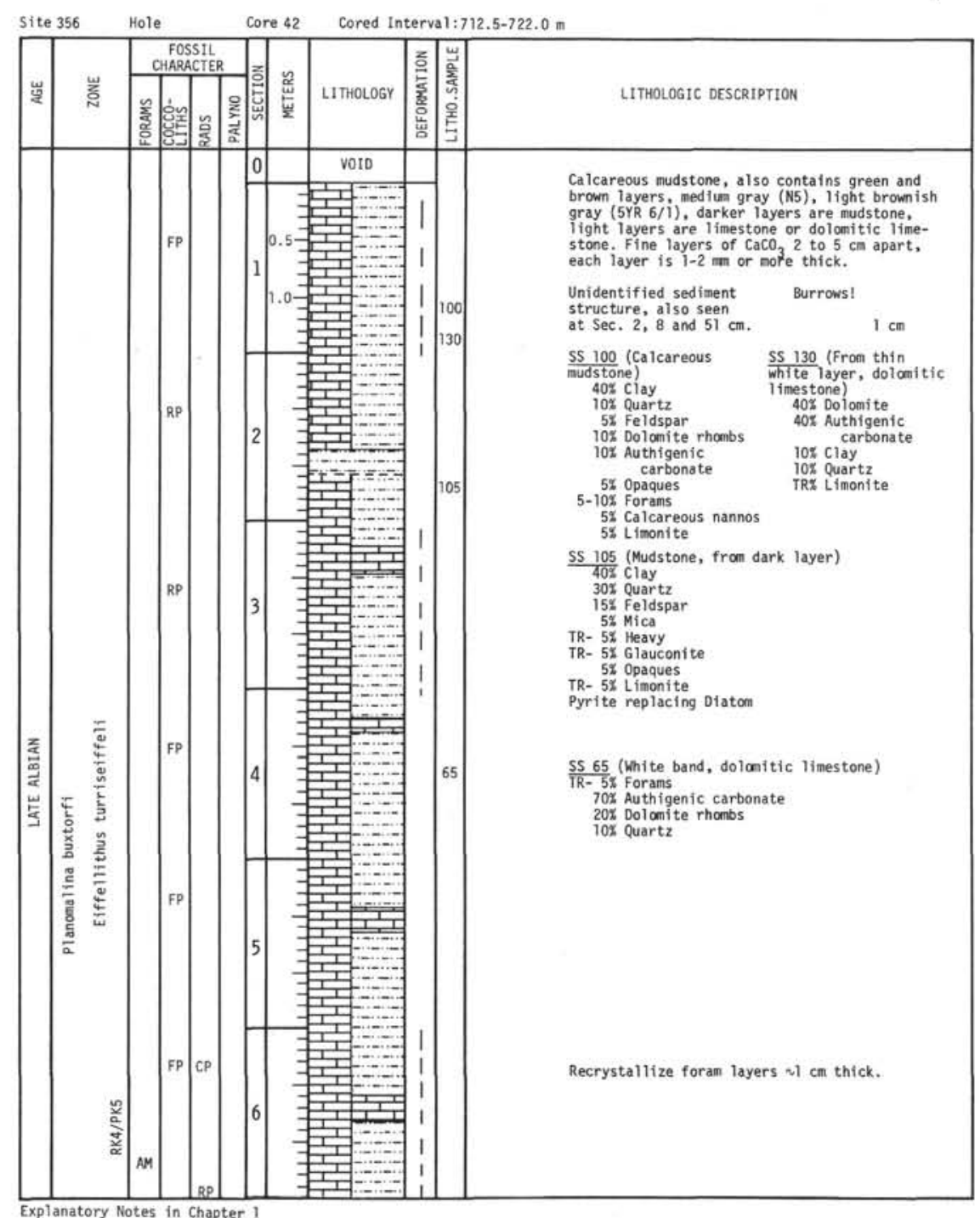




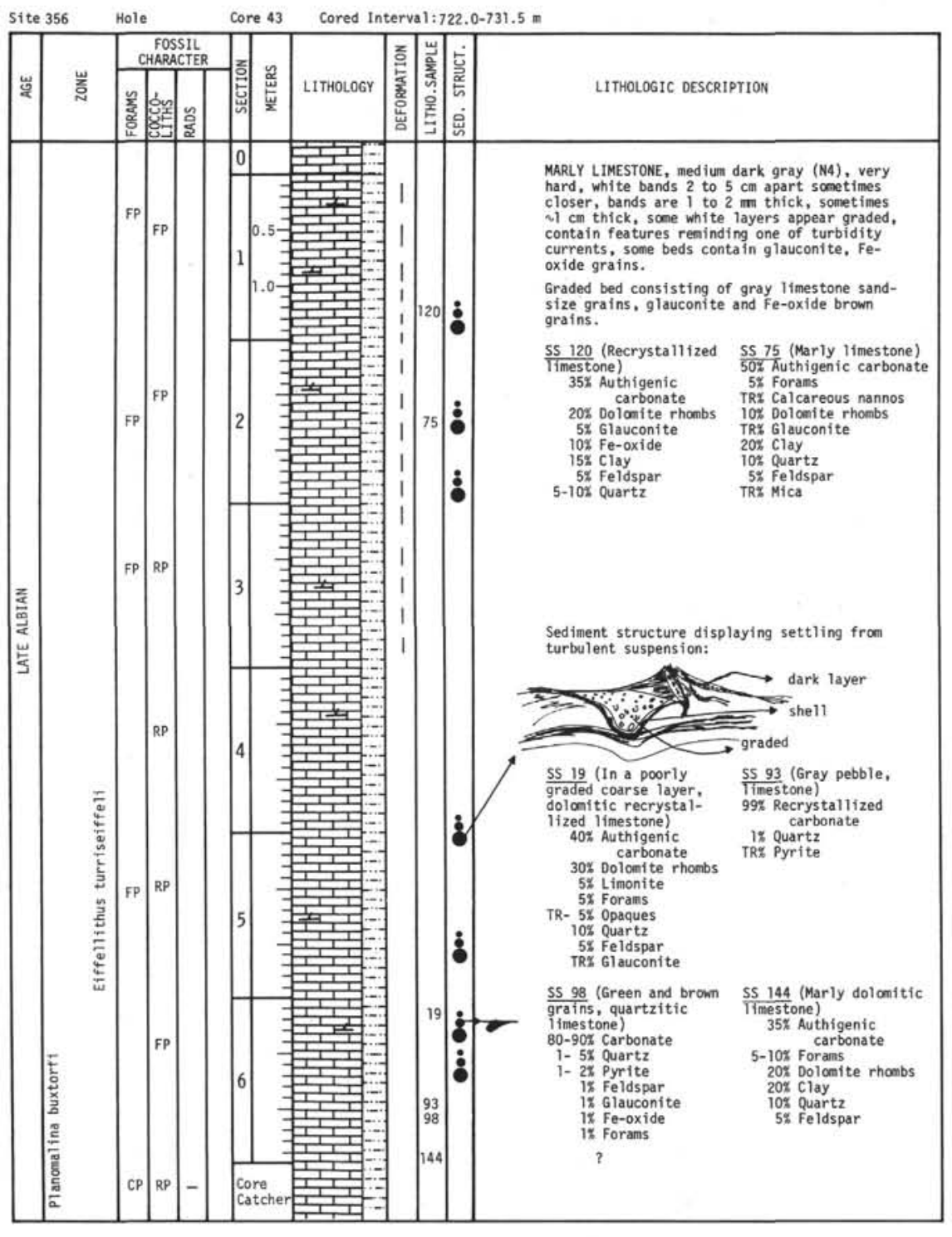

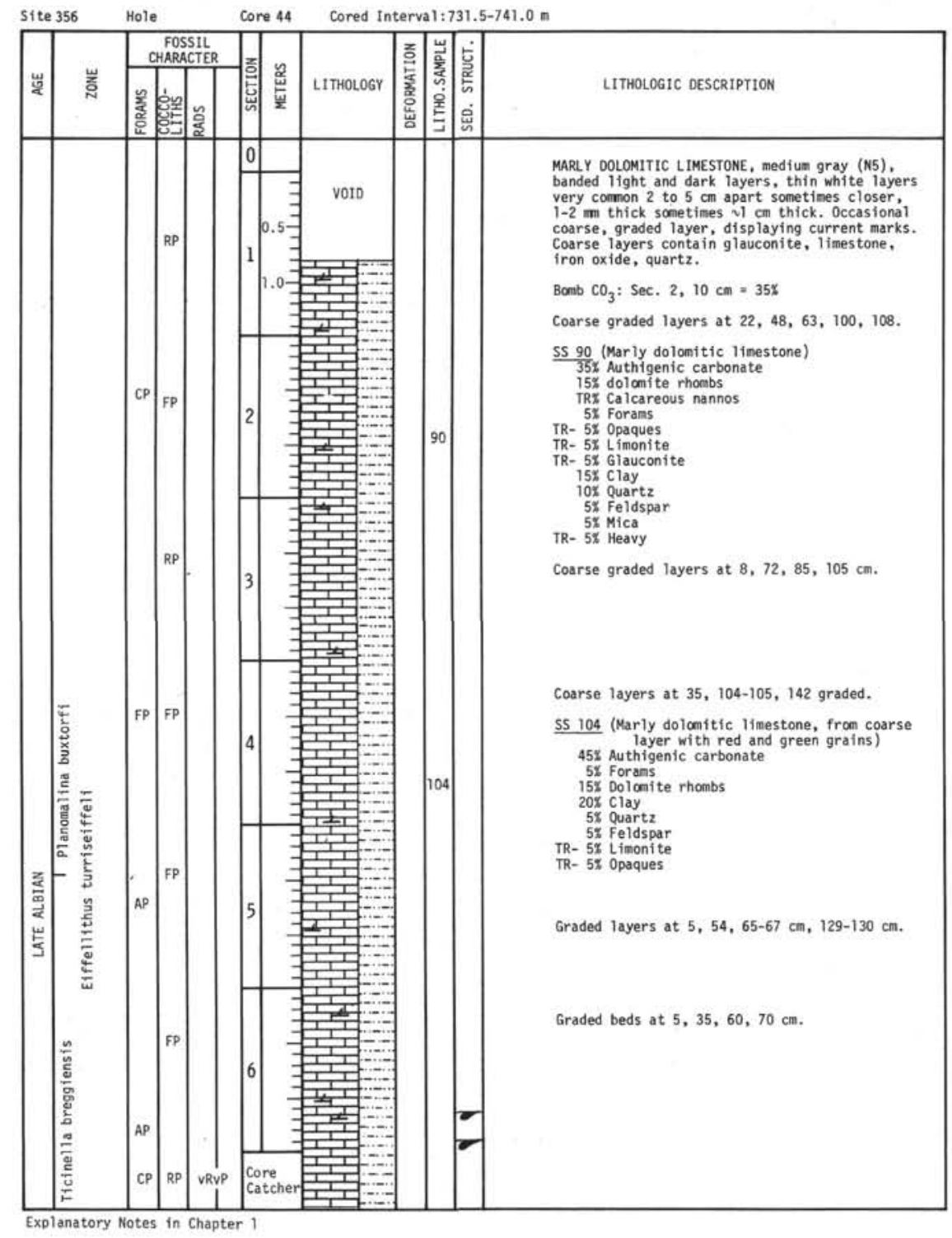




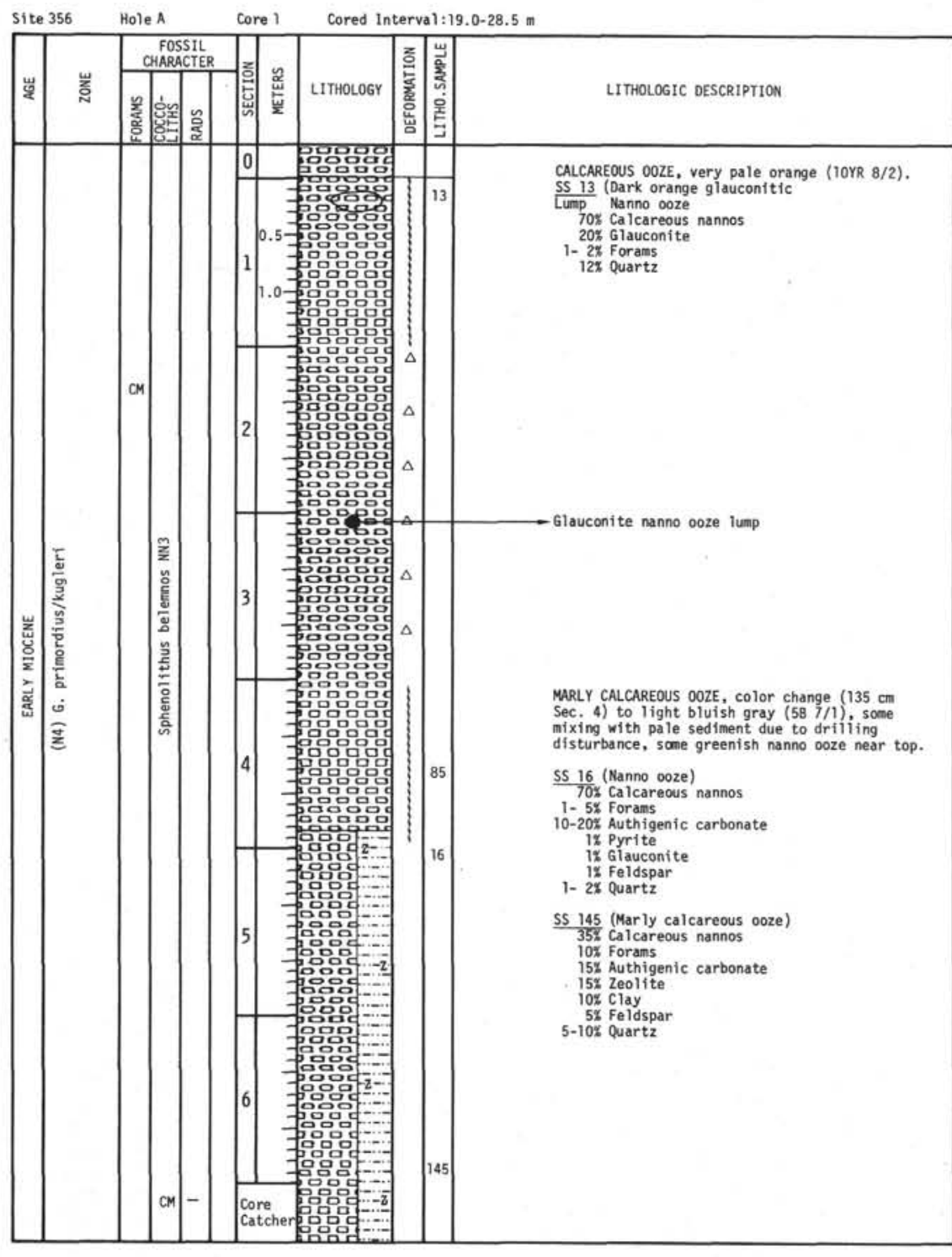

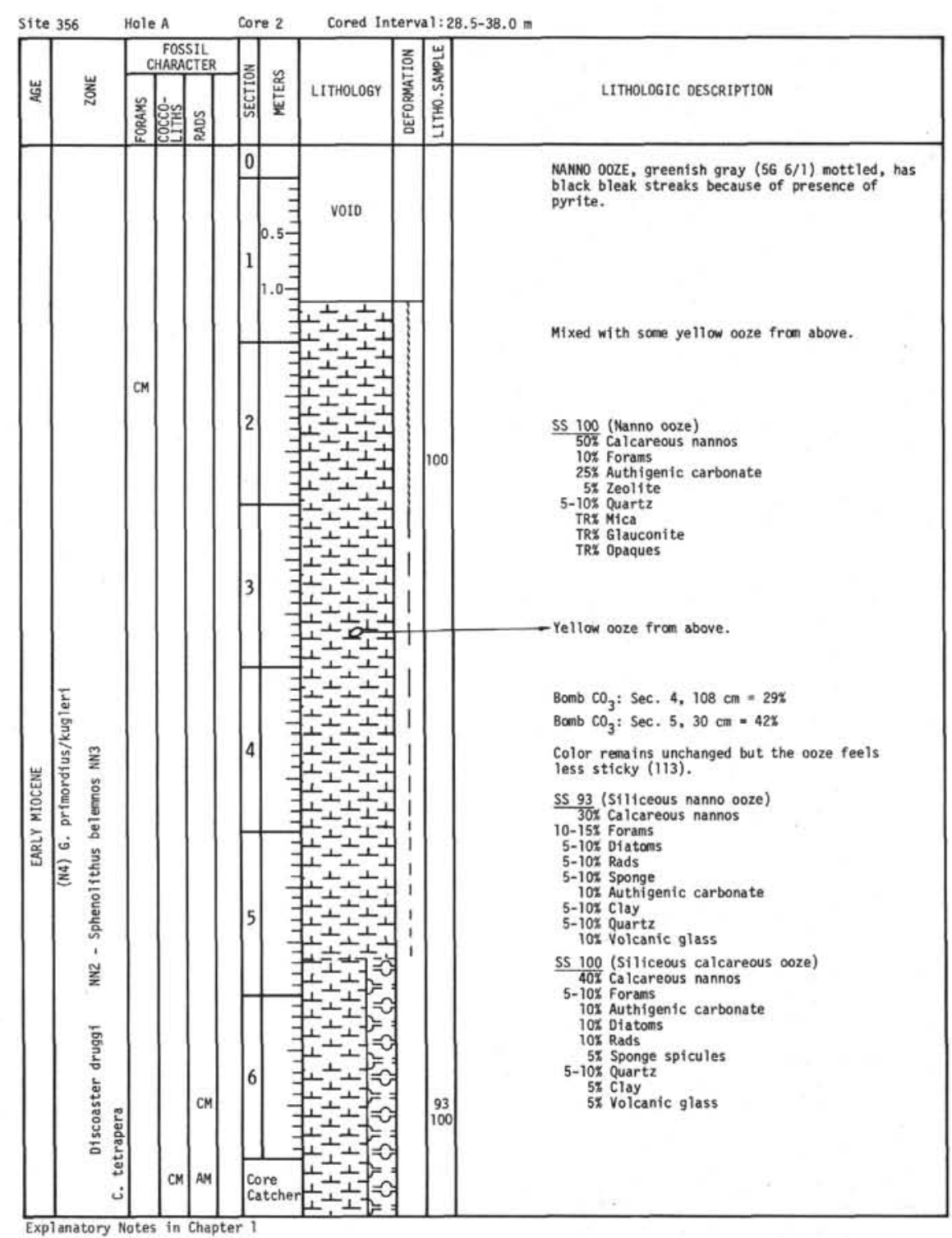


SITE 356: SÃO PAULO PLATEAU

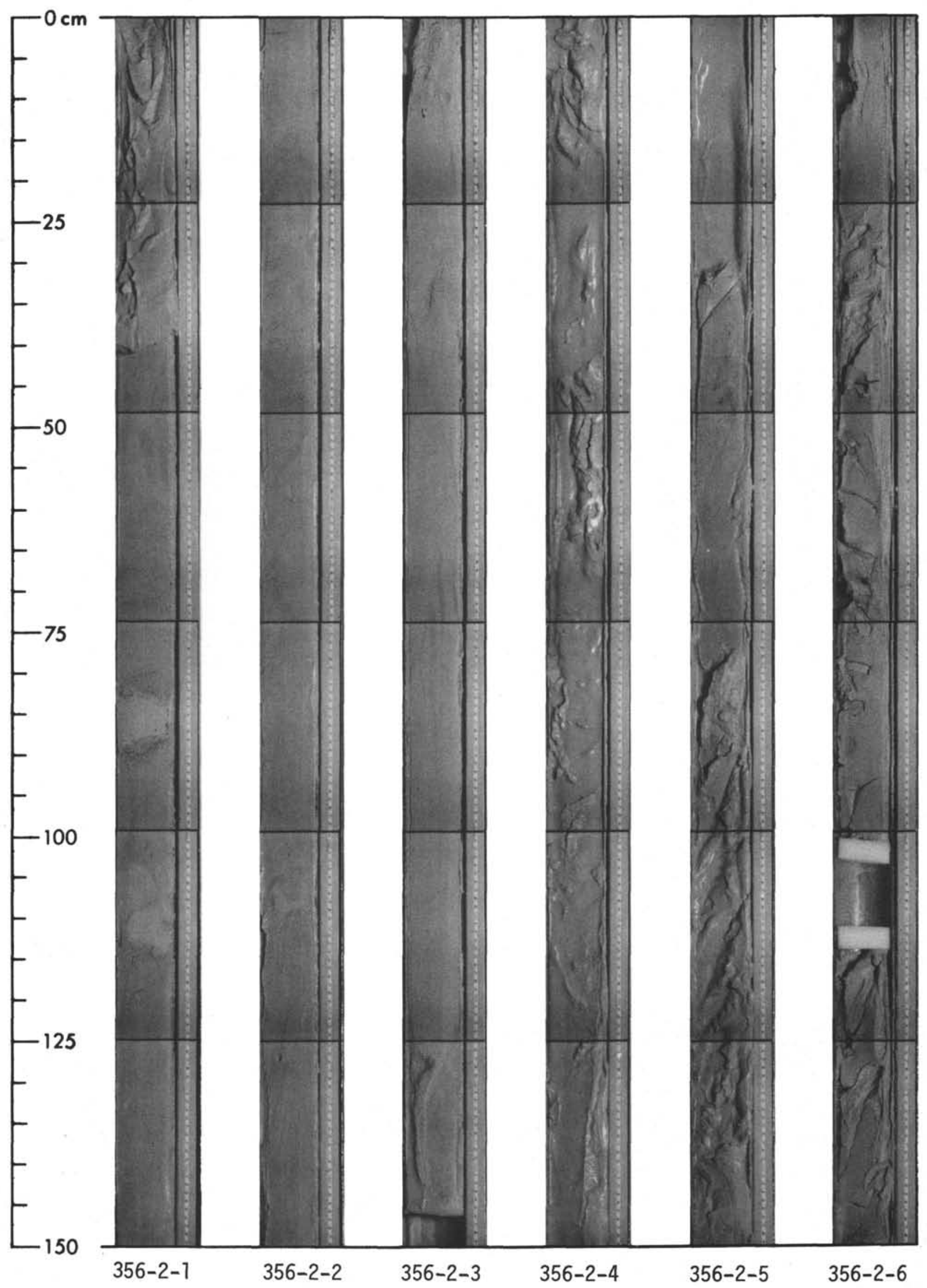


SITE 356: SÃO PAULO PLATEAU

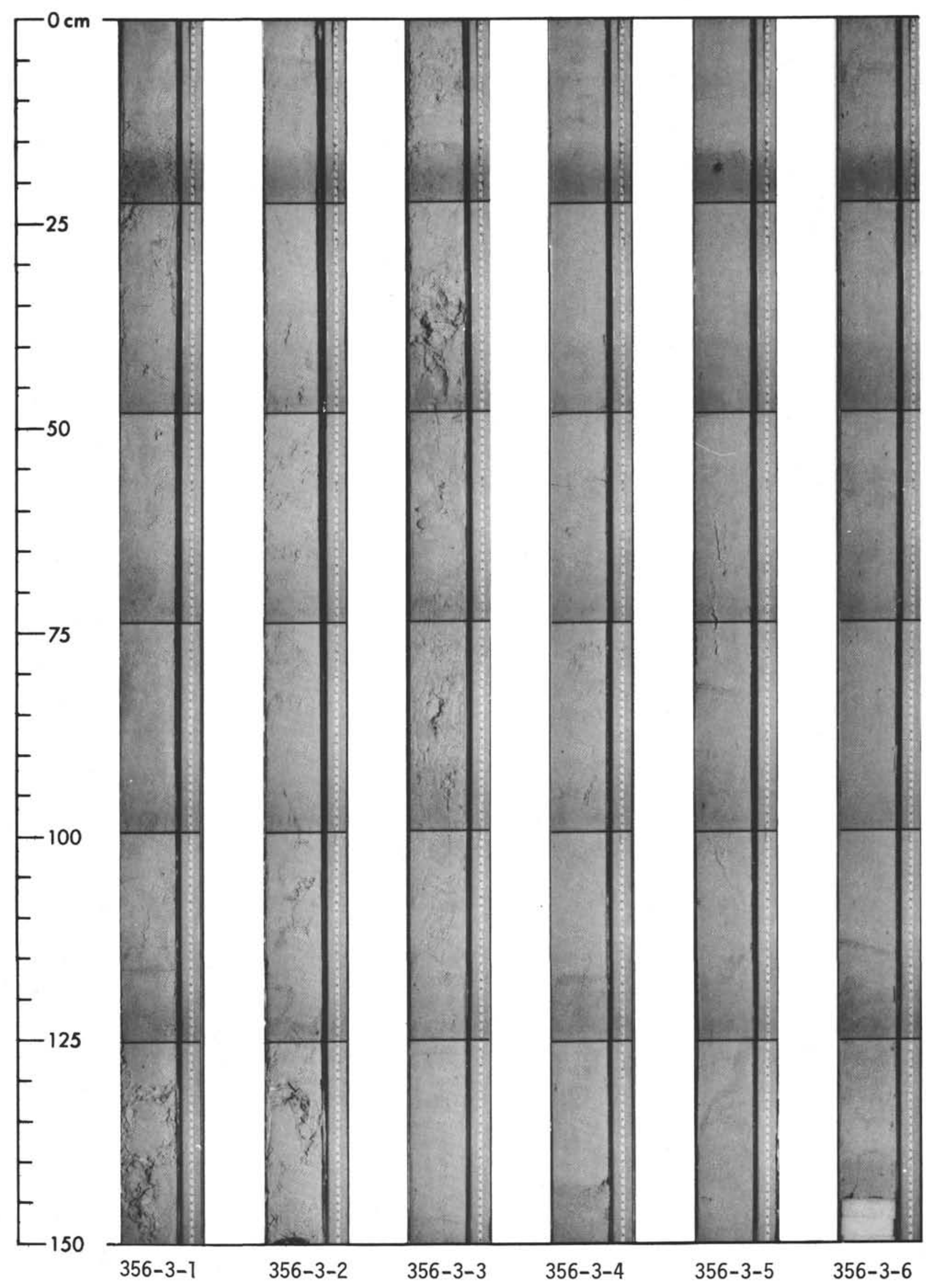




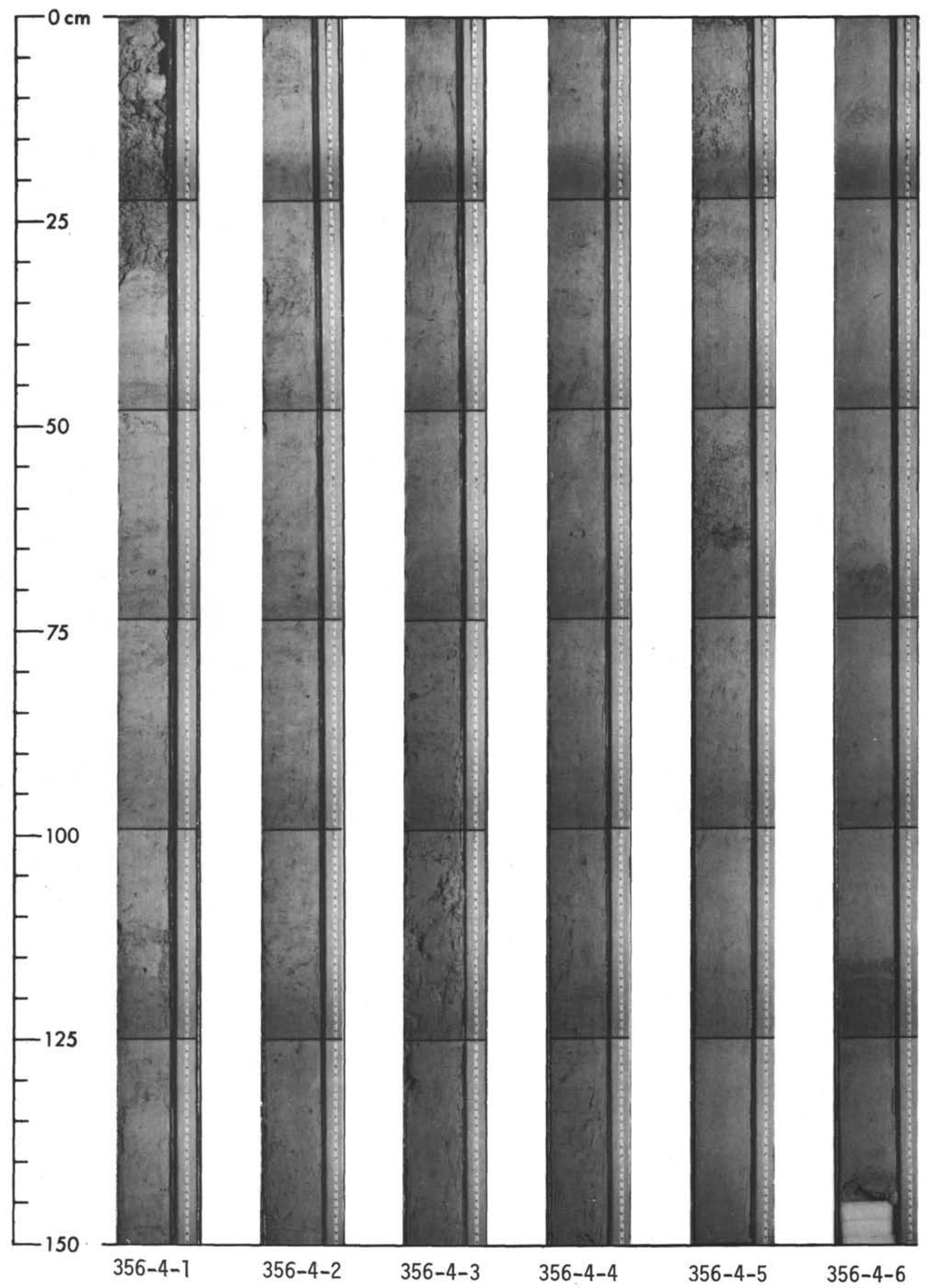


SITE 356: SÃO PAULO PLATEAU

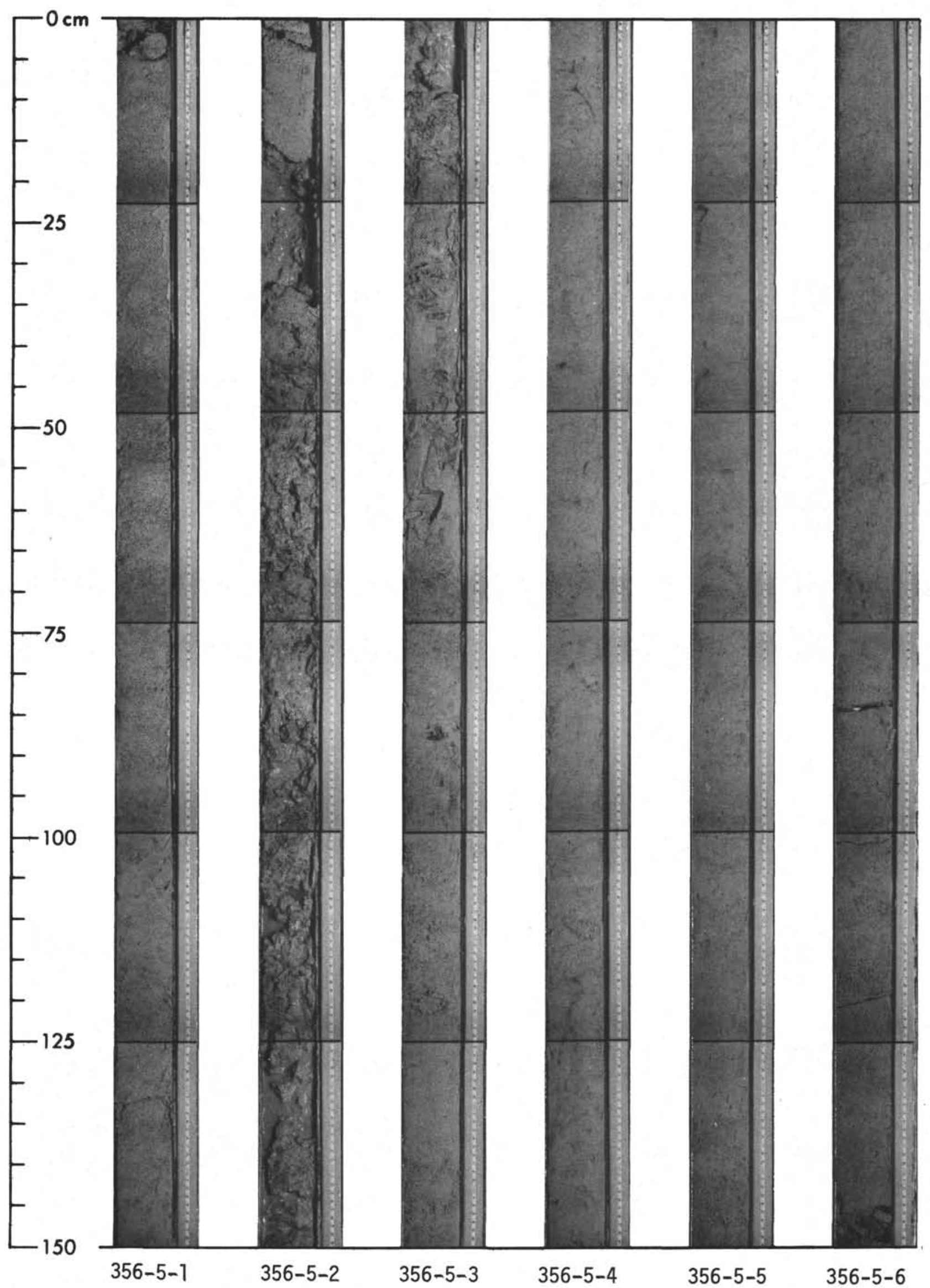




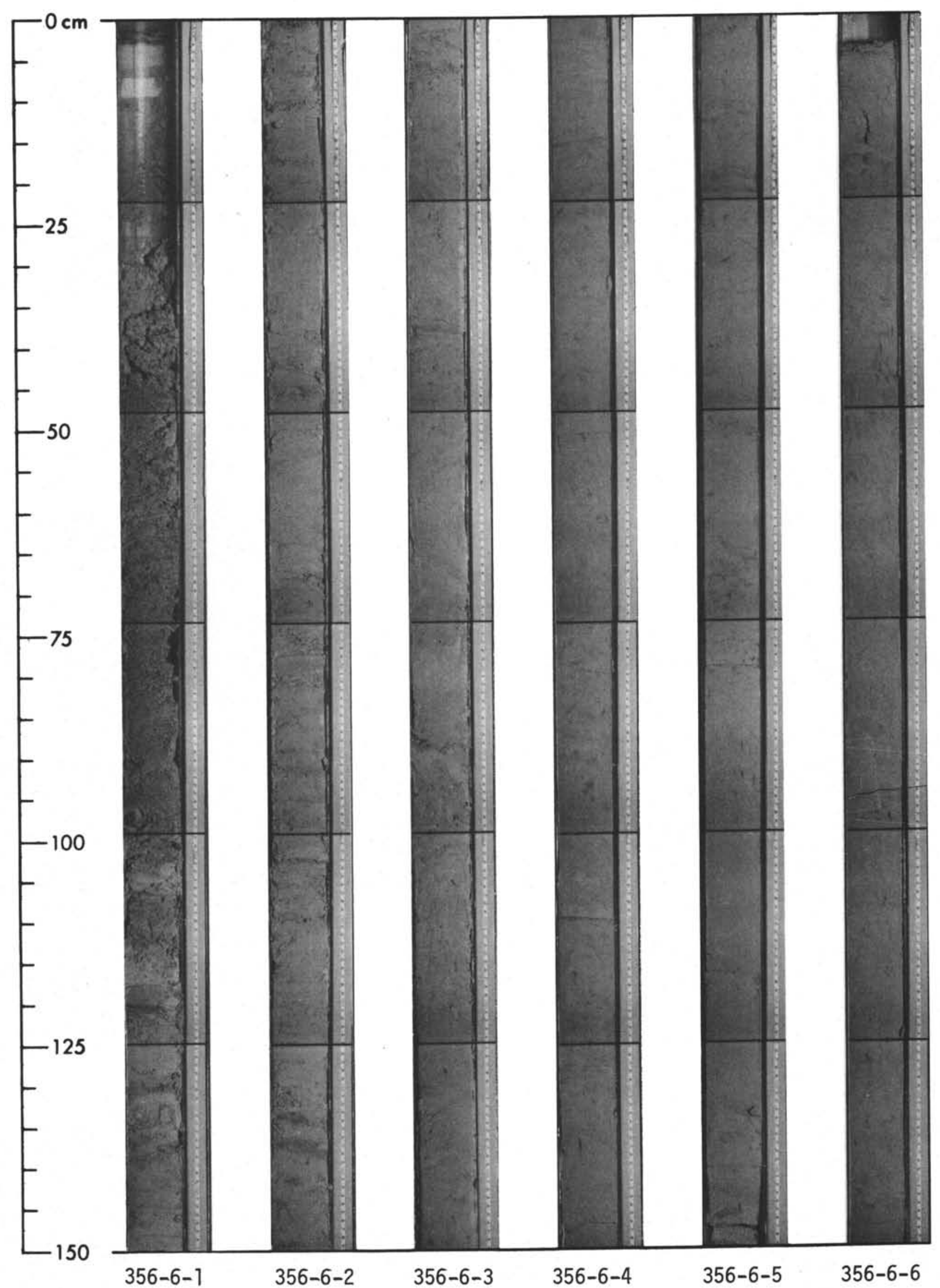


SITE 356: SÃO PAULO PLATEAU

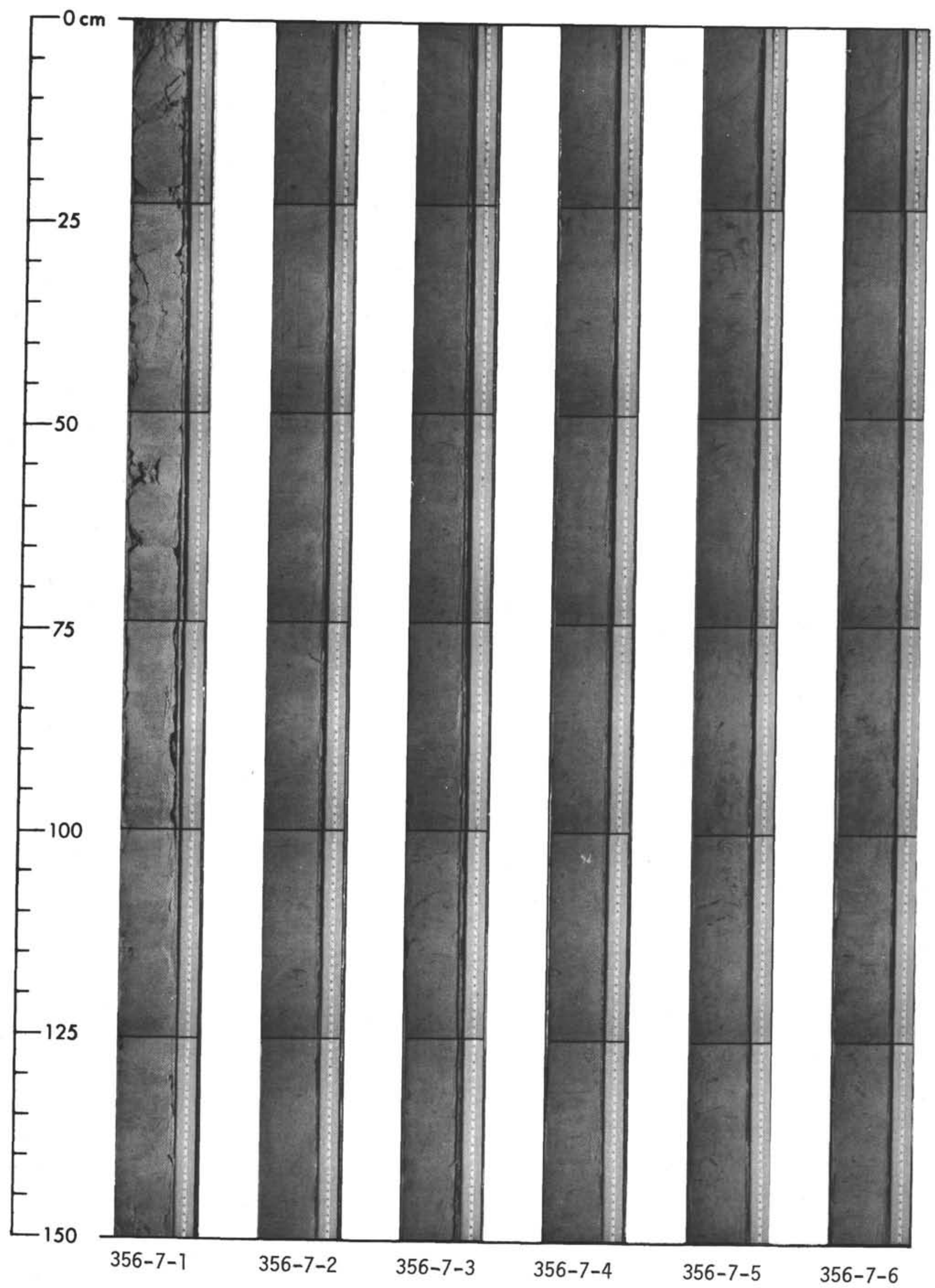




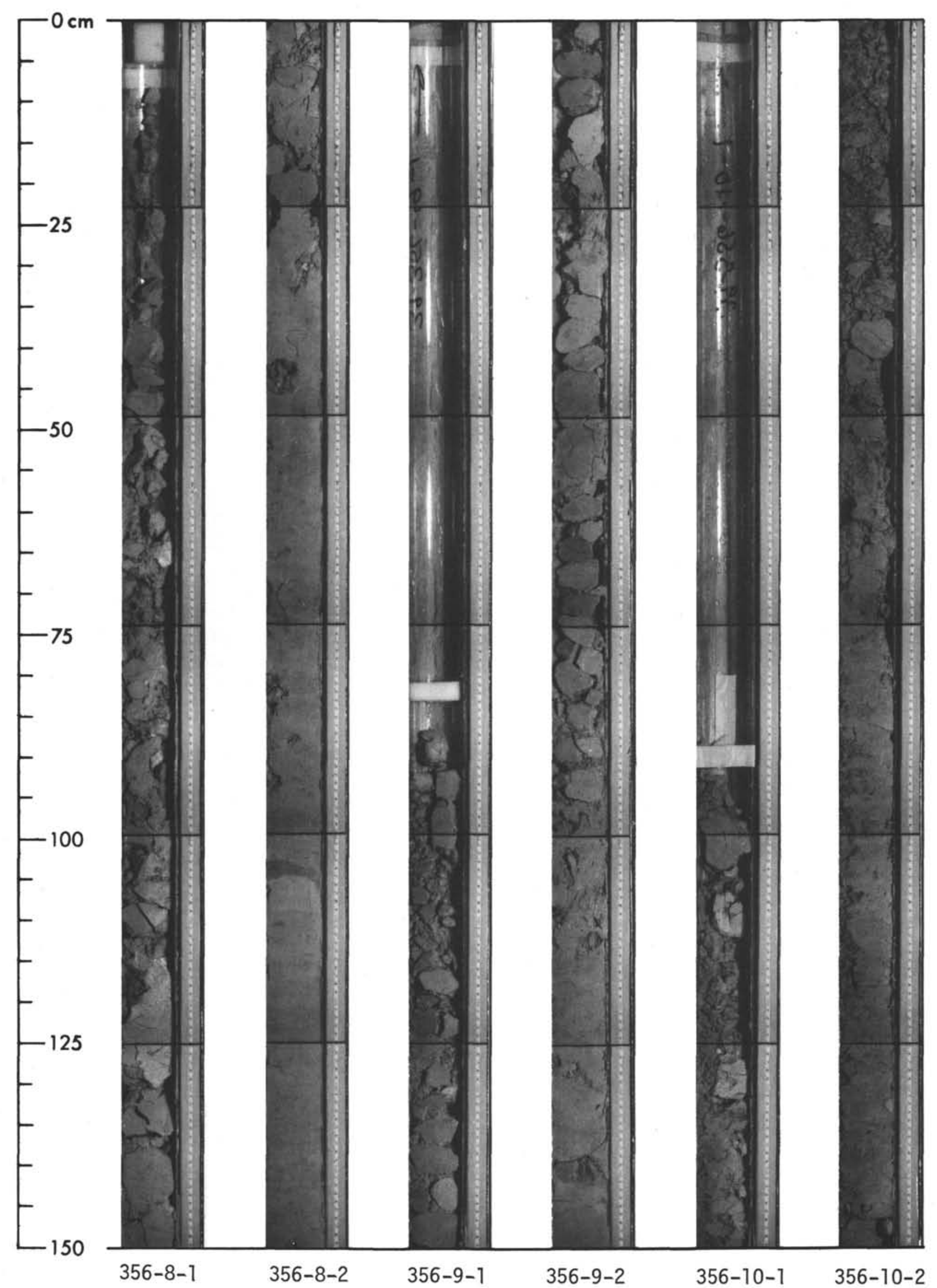


SITE 356: SÃO PAULO PLATEAU

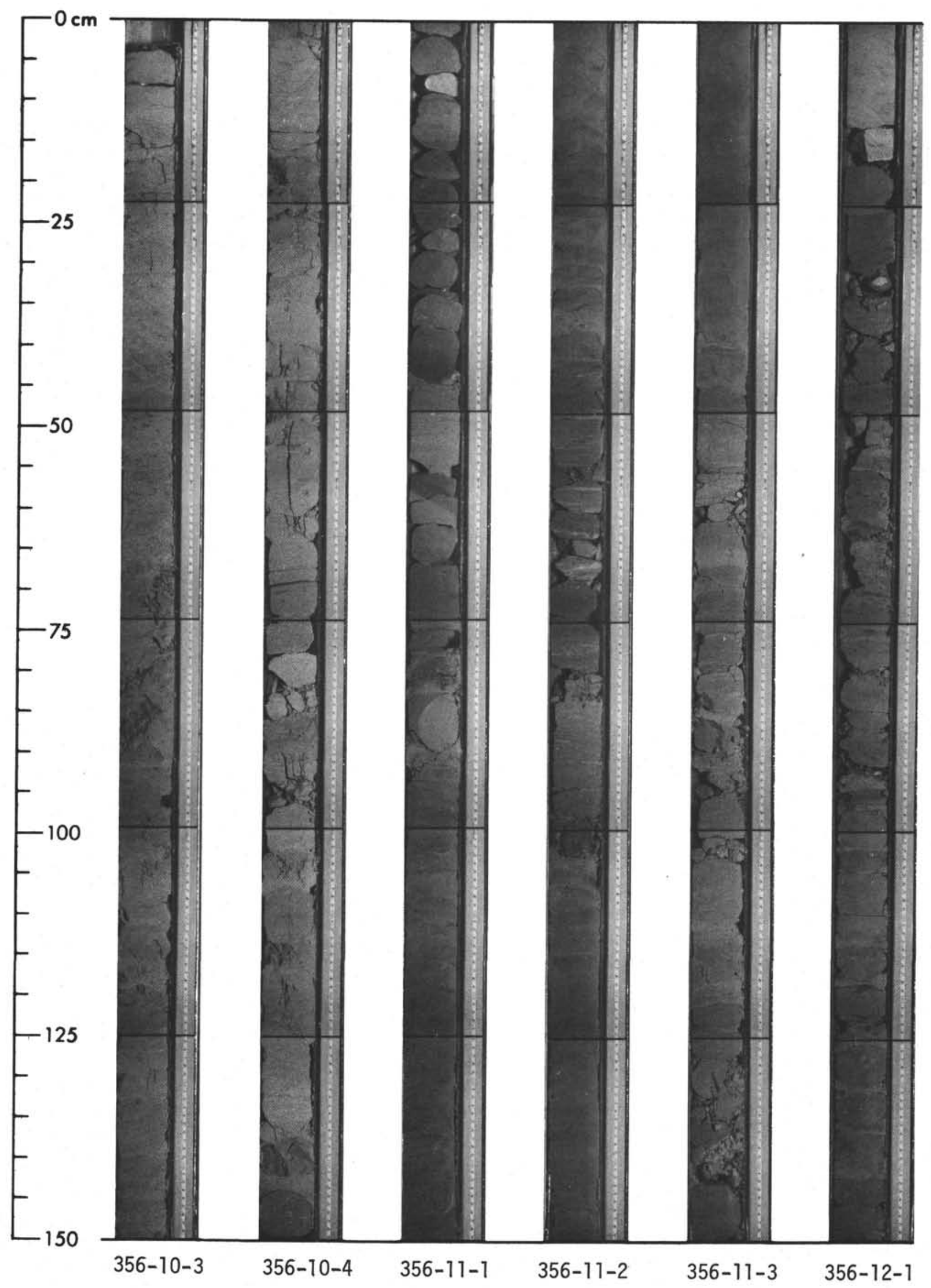


SITE 356: SÃO PAULO PLATEAU

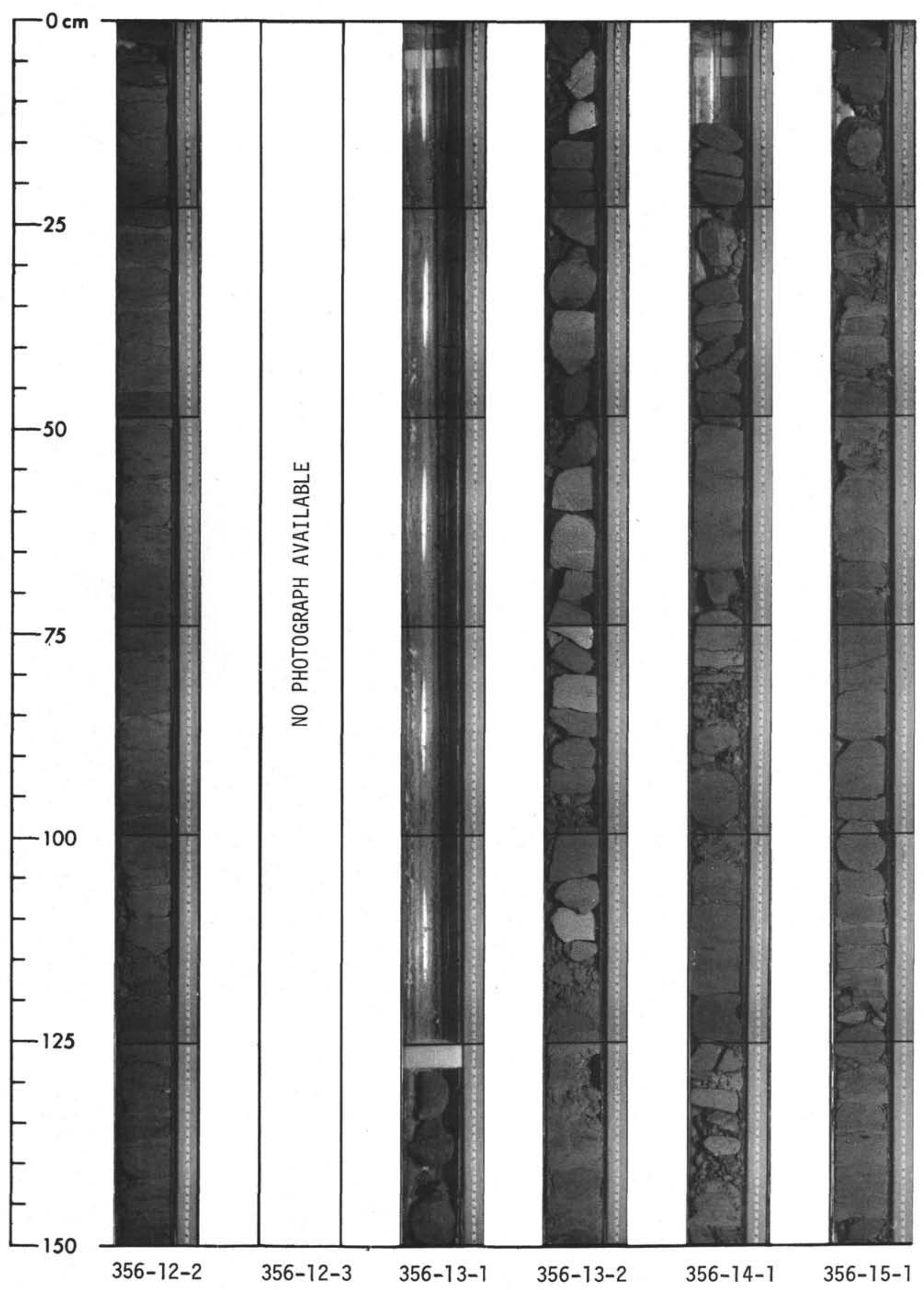


SITE 356: SÃO PAULO PLATEAU

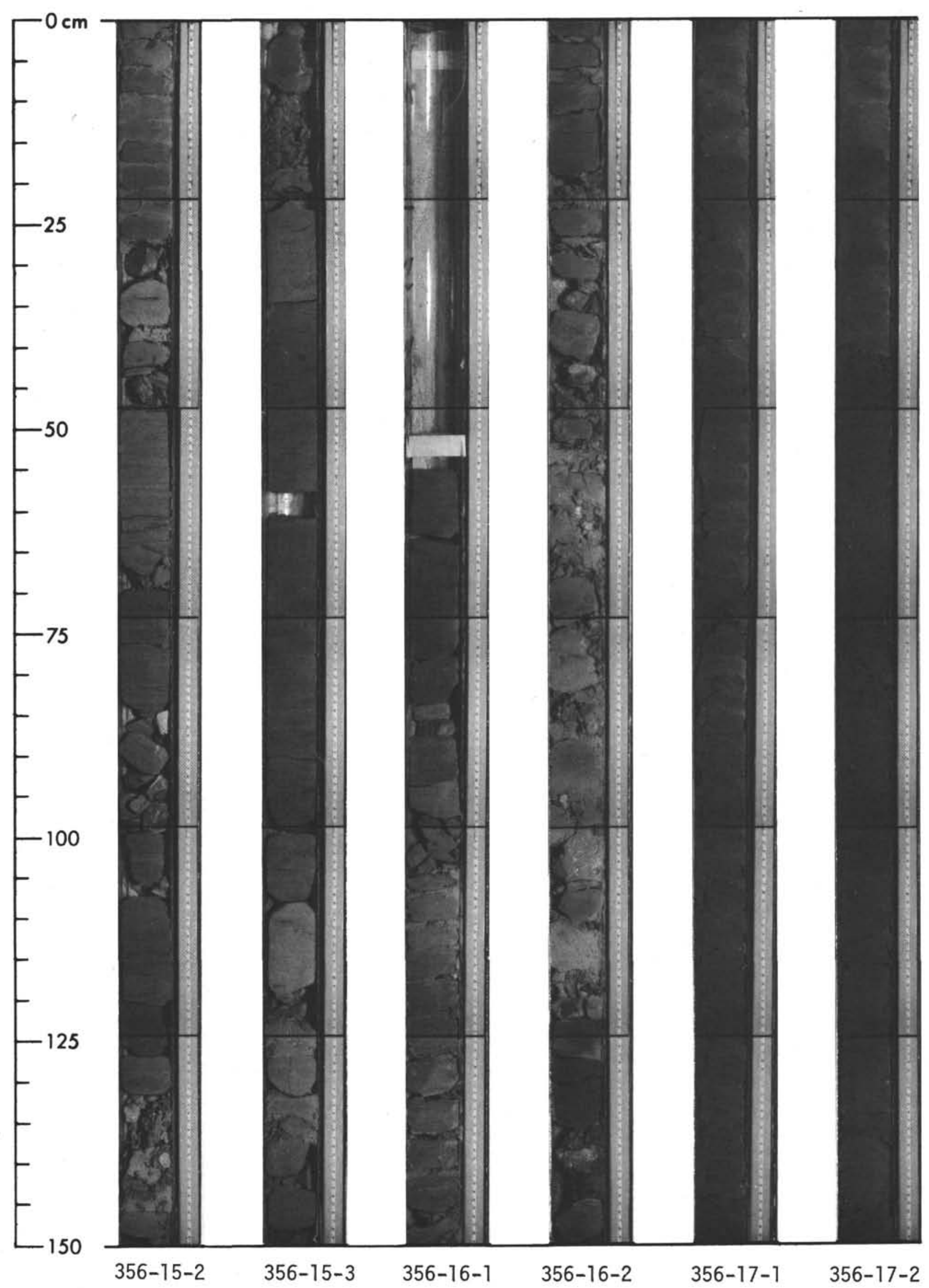


SITE 356: SÃO PAULO PLATEAU

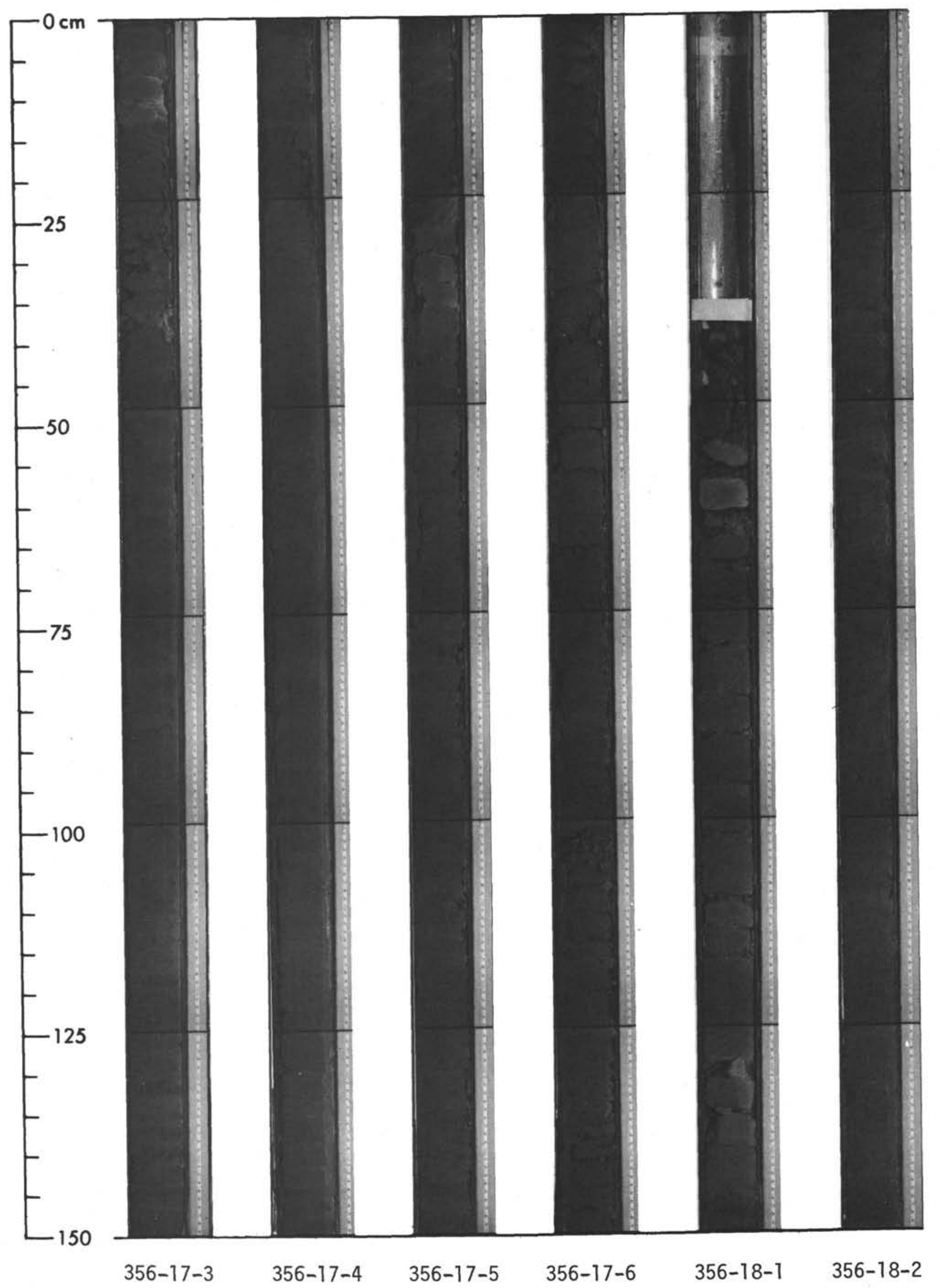


SITE 356: SÃO PAULO PLATEAU

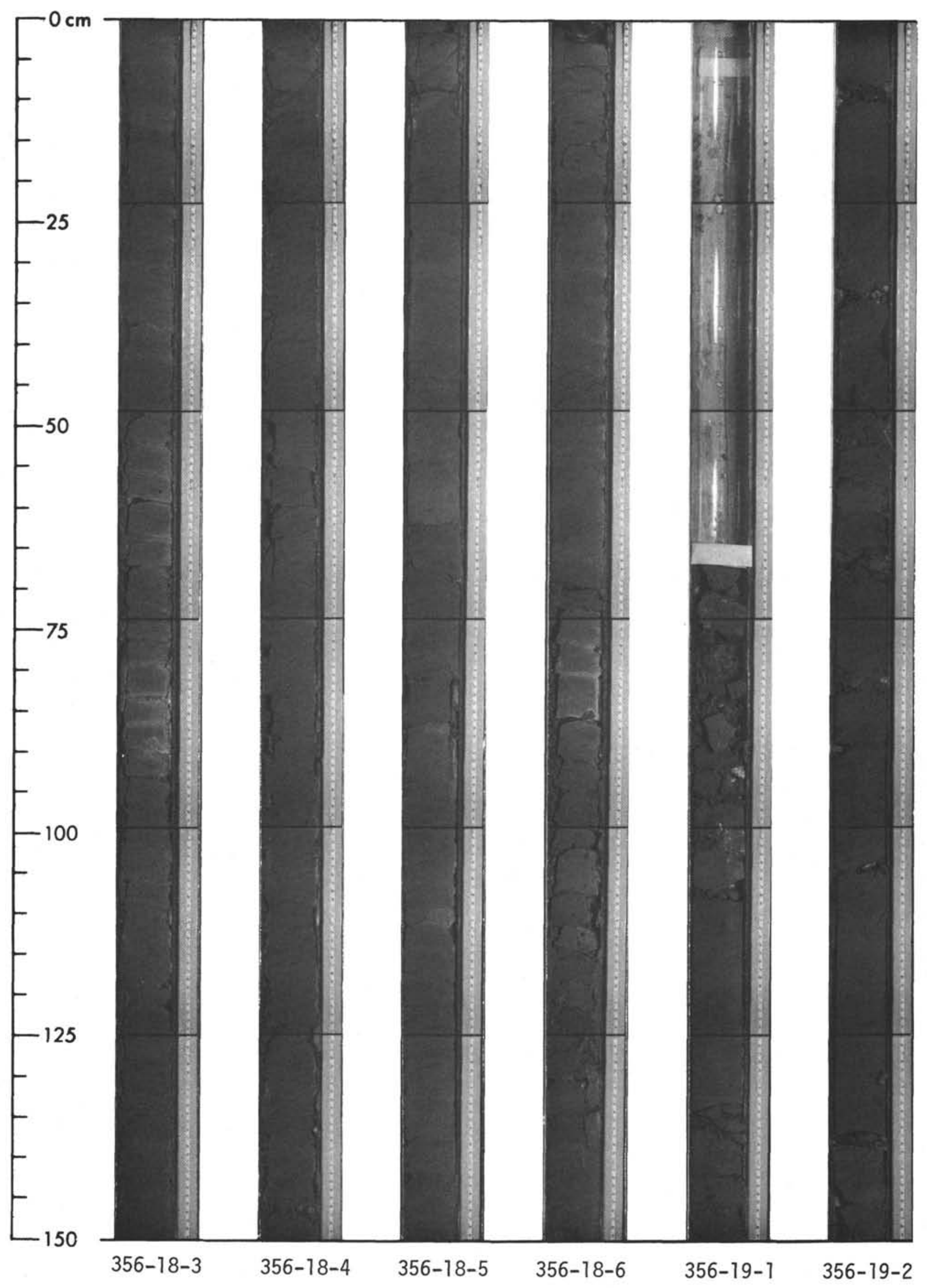


SITE 356: SÃO PAULO PLATEAU

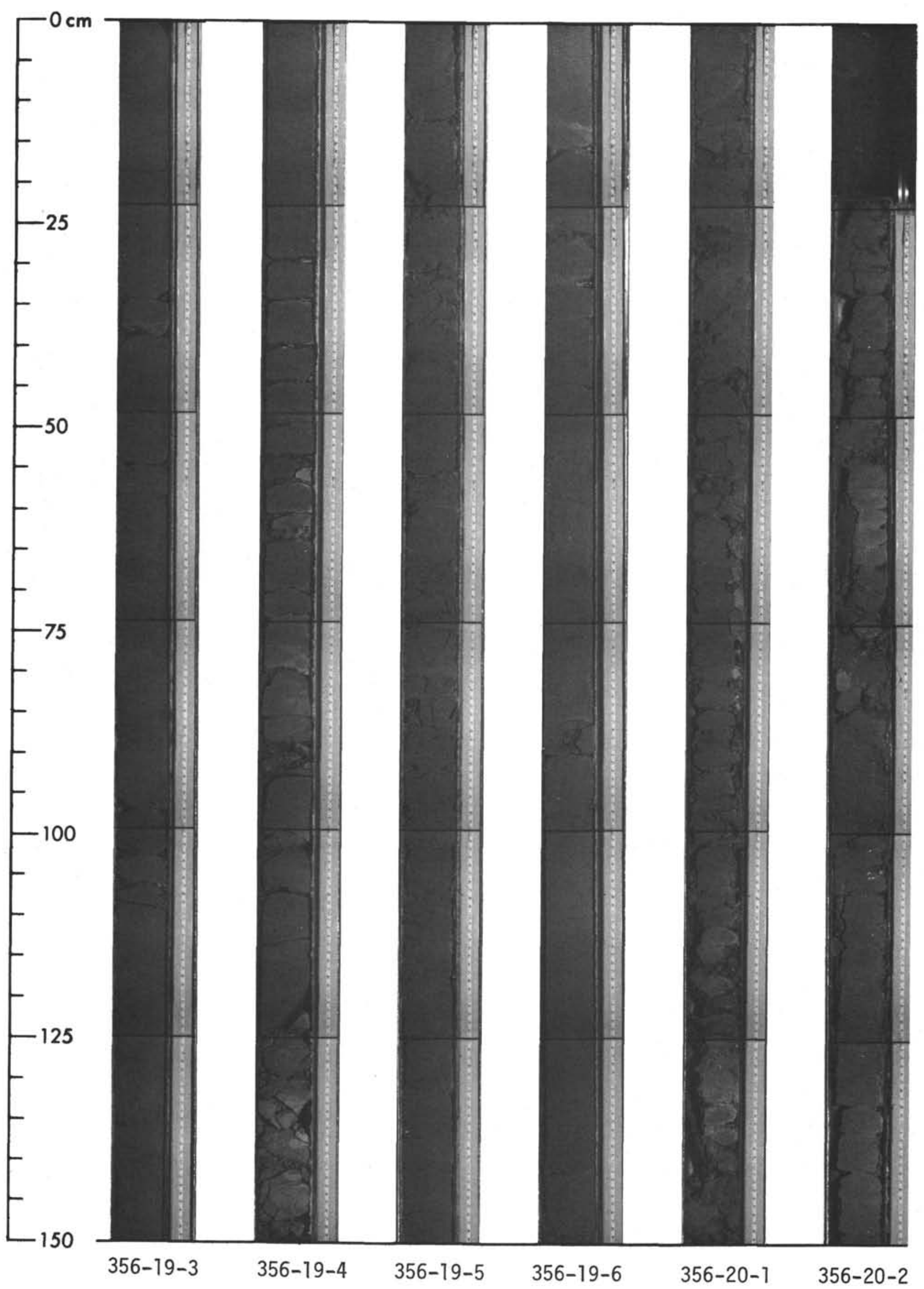


SITE 356: SÃO PAULO PLATEAU

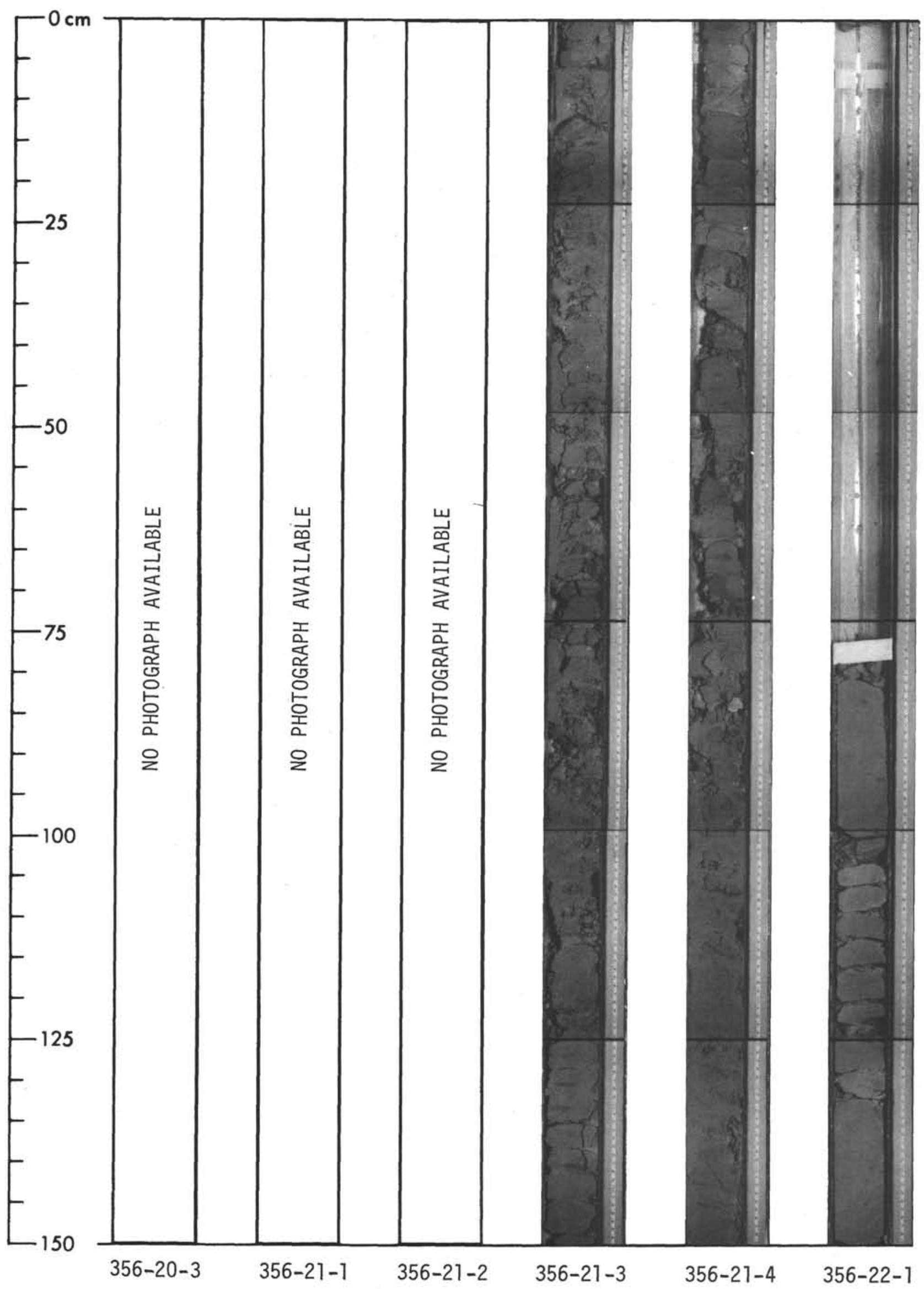




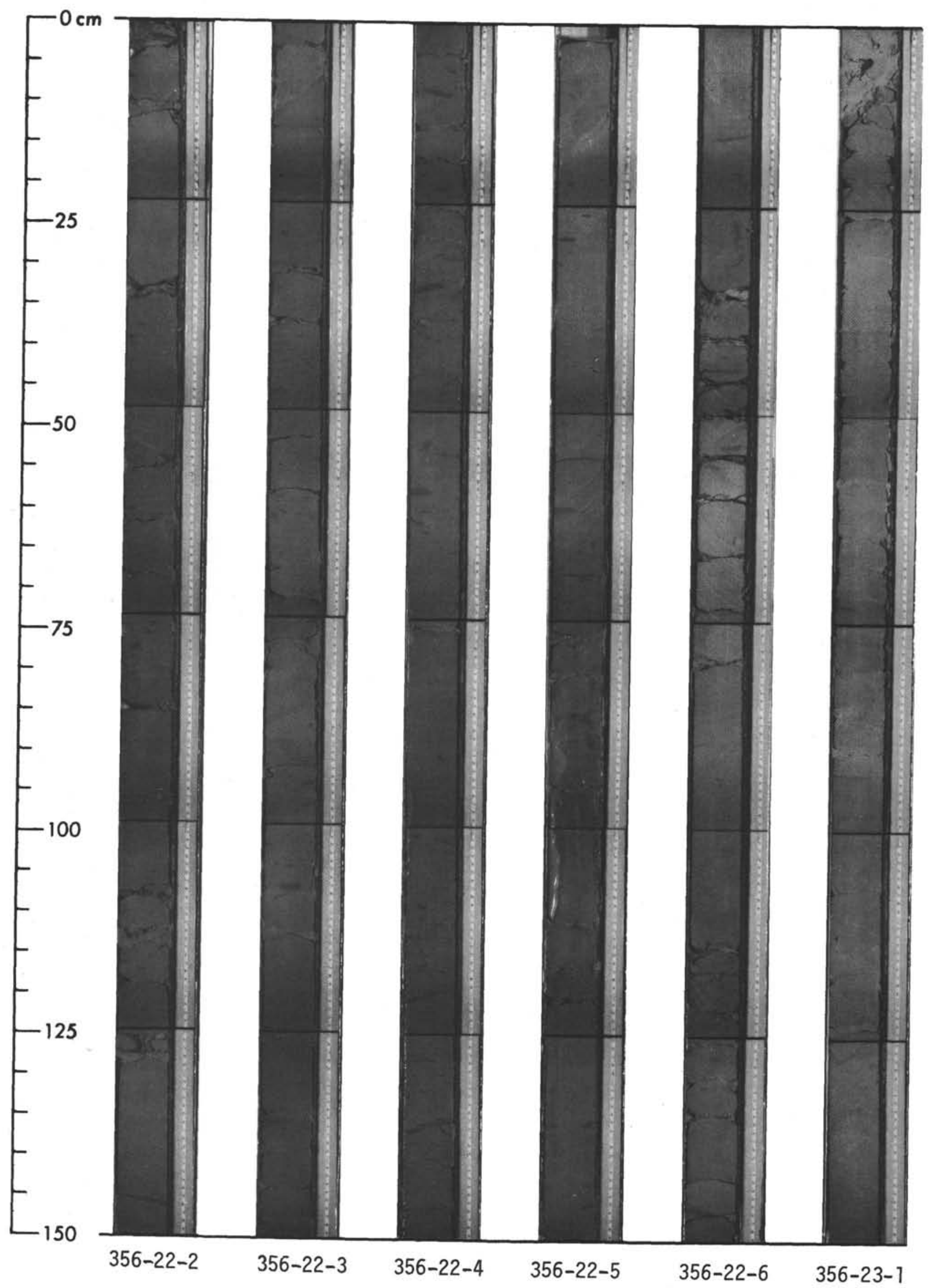


SITE 356: SÃO PAULO PLATEAU

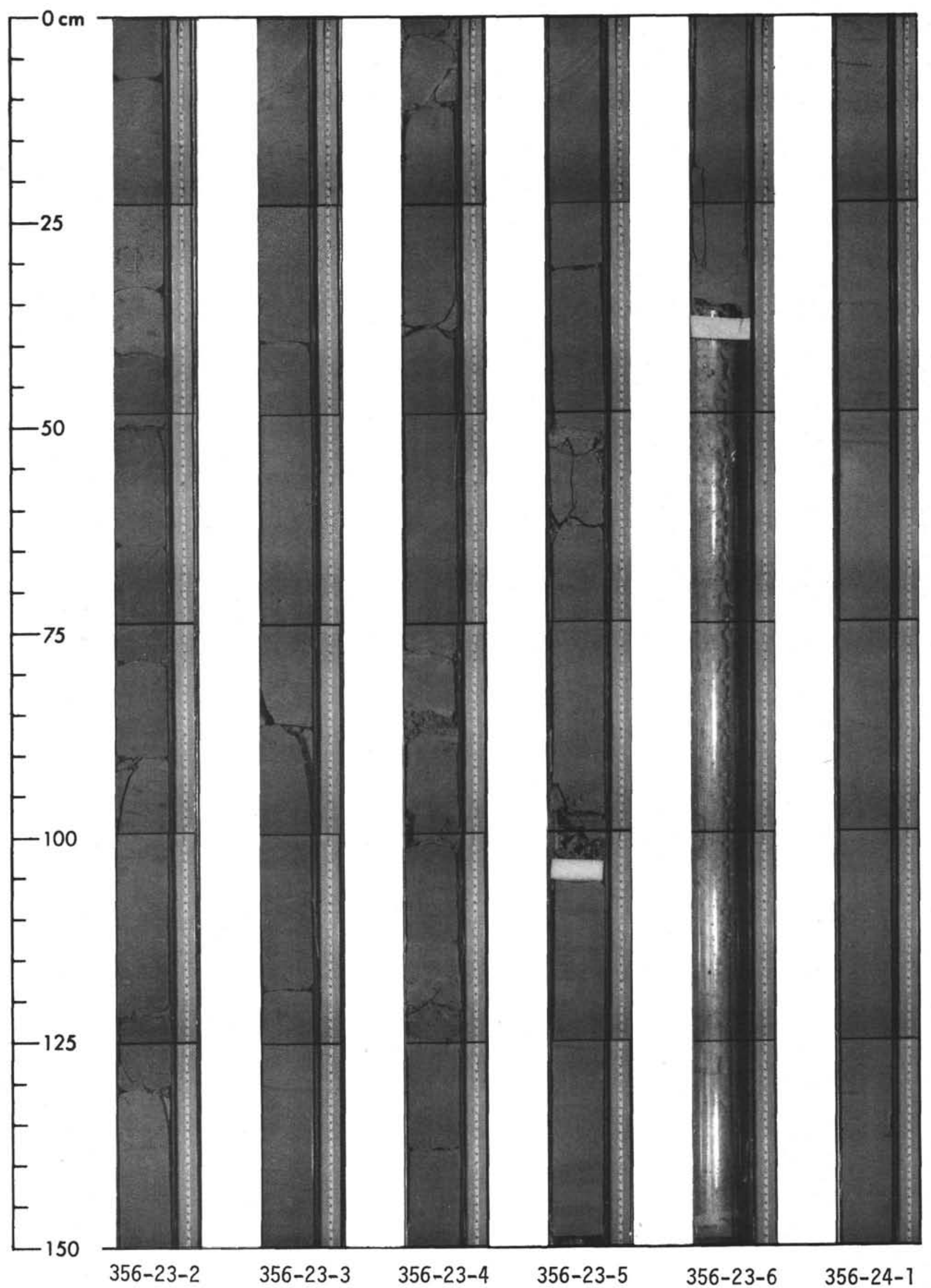




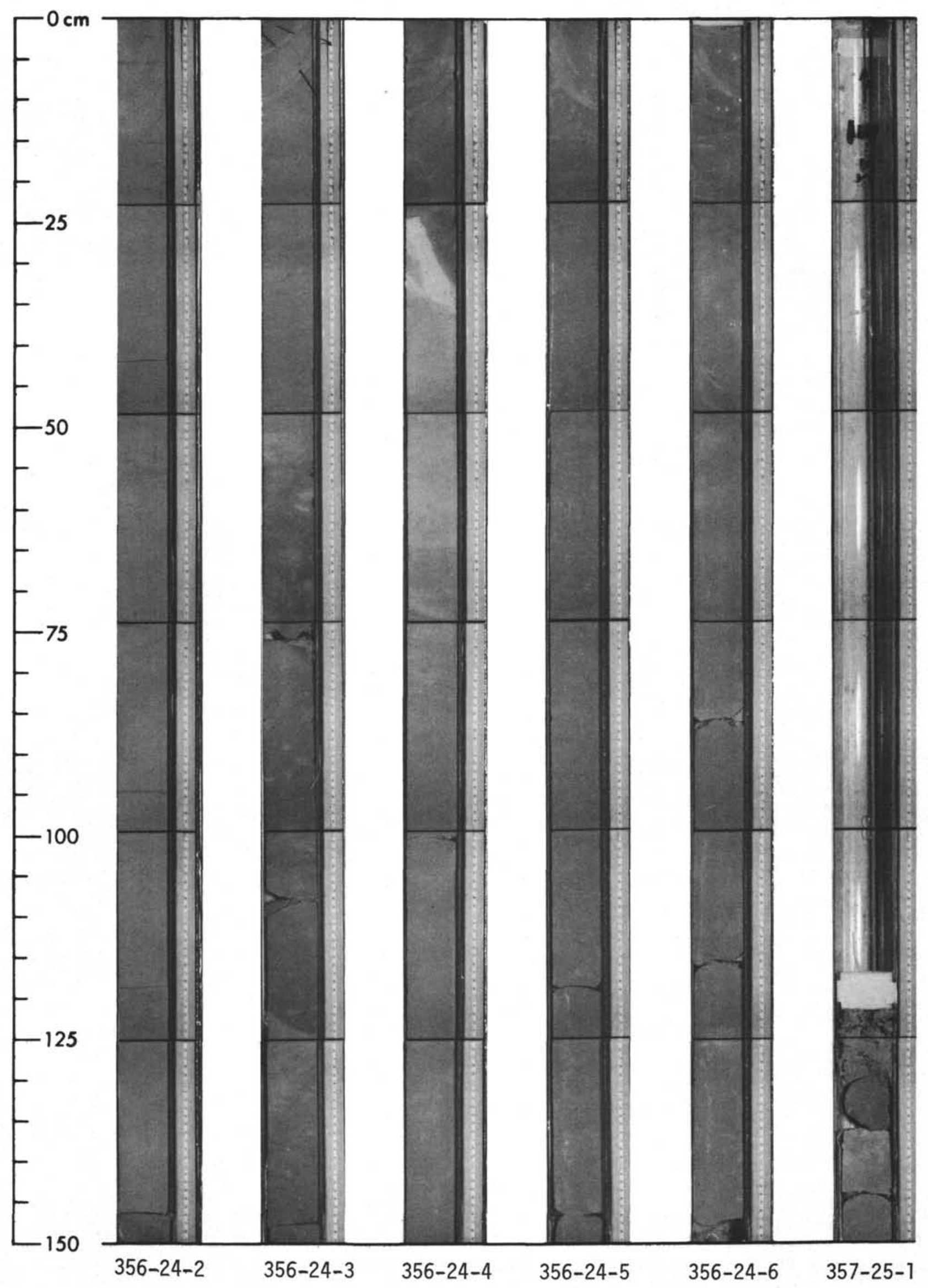


SITE 356: SÃO PAULO PLATEAU

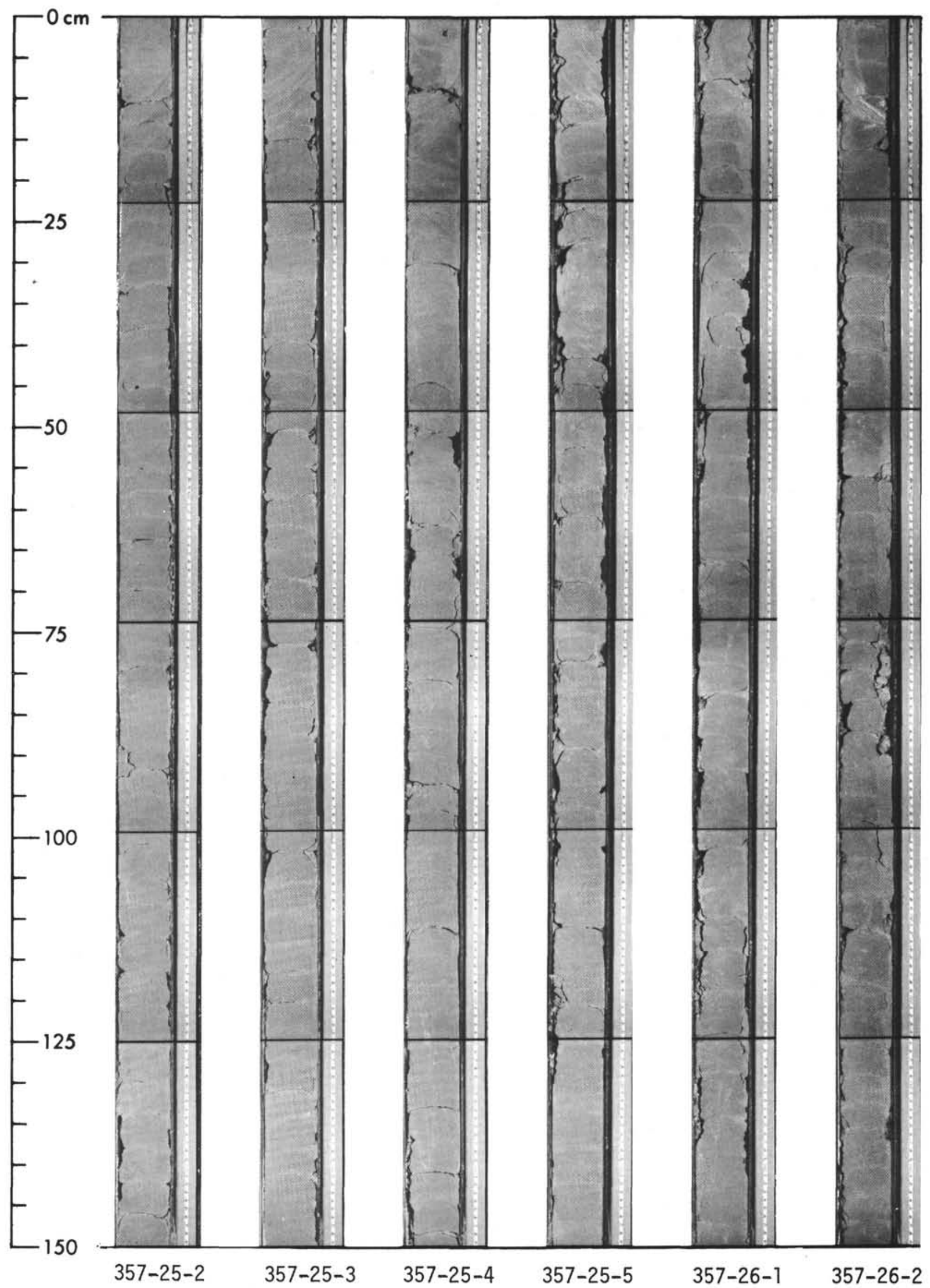


SITE 356: ST̃O PAULO PLATEAU

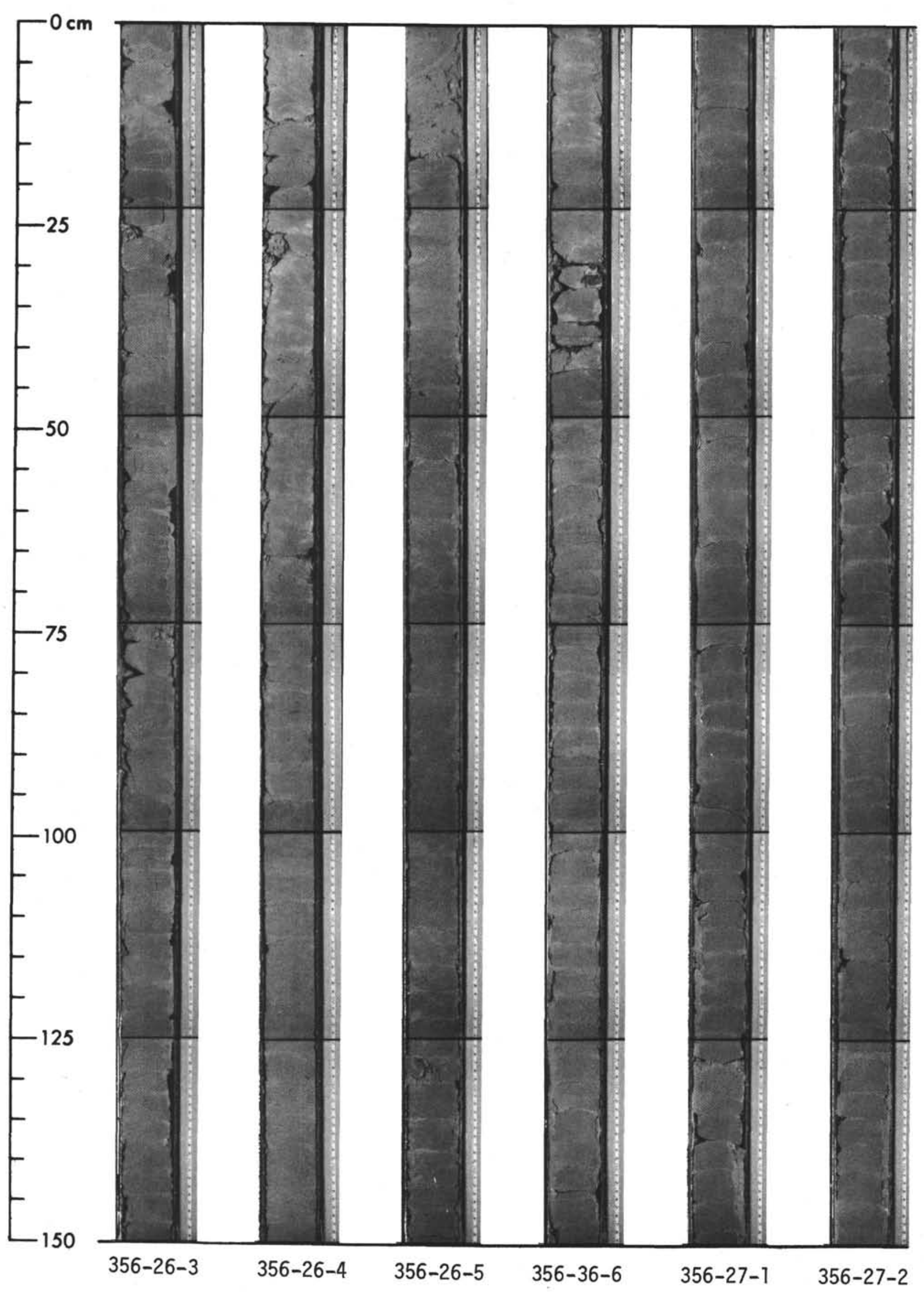


SITE 356: SÃO PAULO PLATEAU

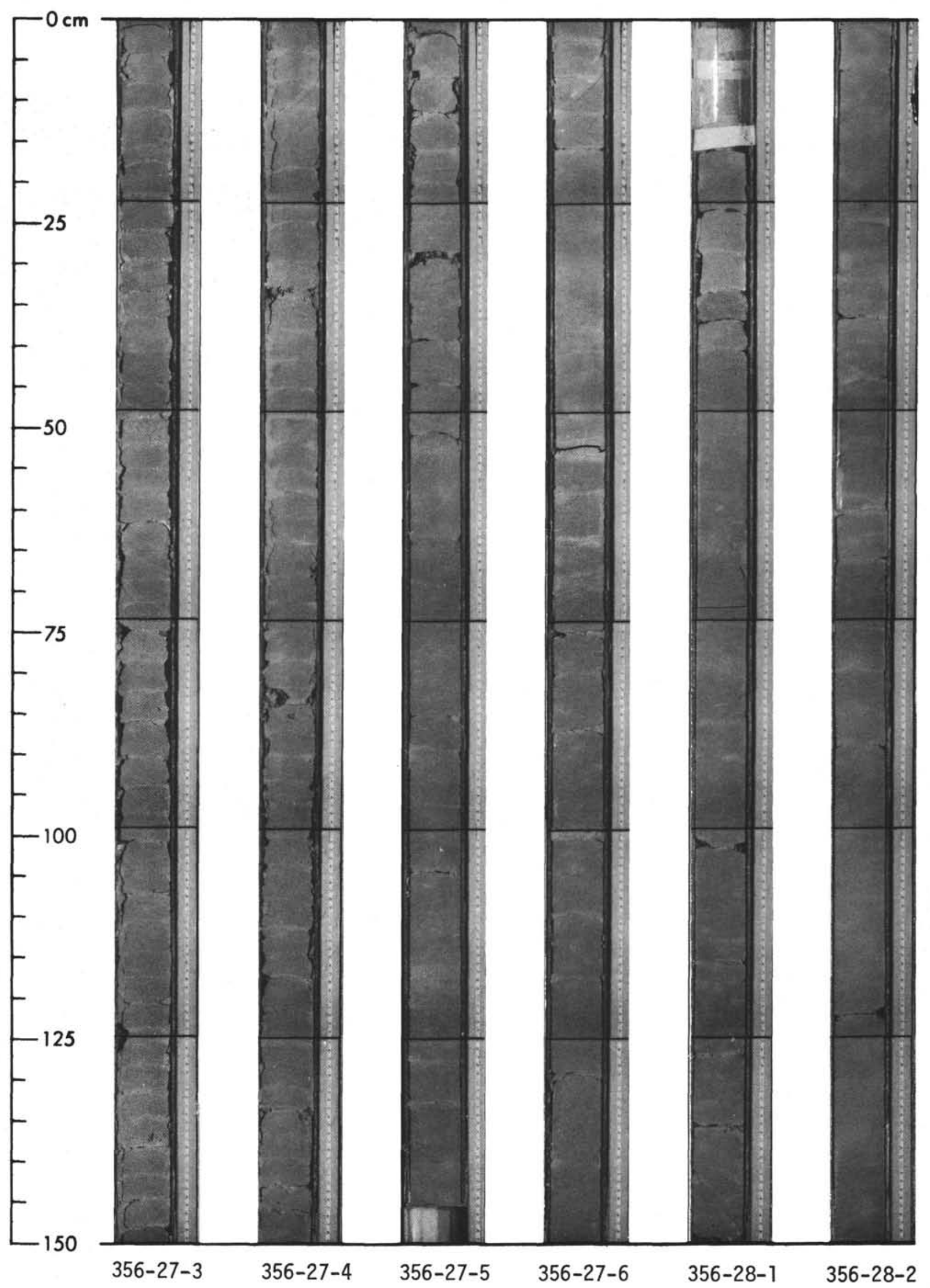


SITE 356: SÃO PAULO PLATEAU

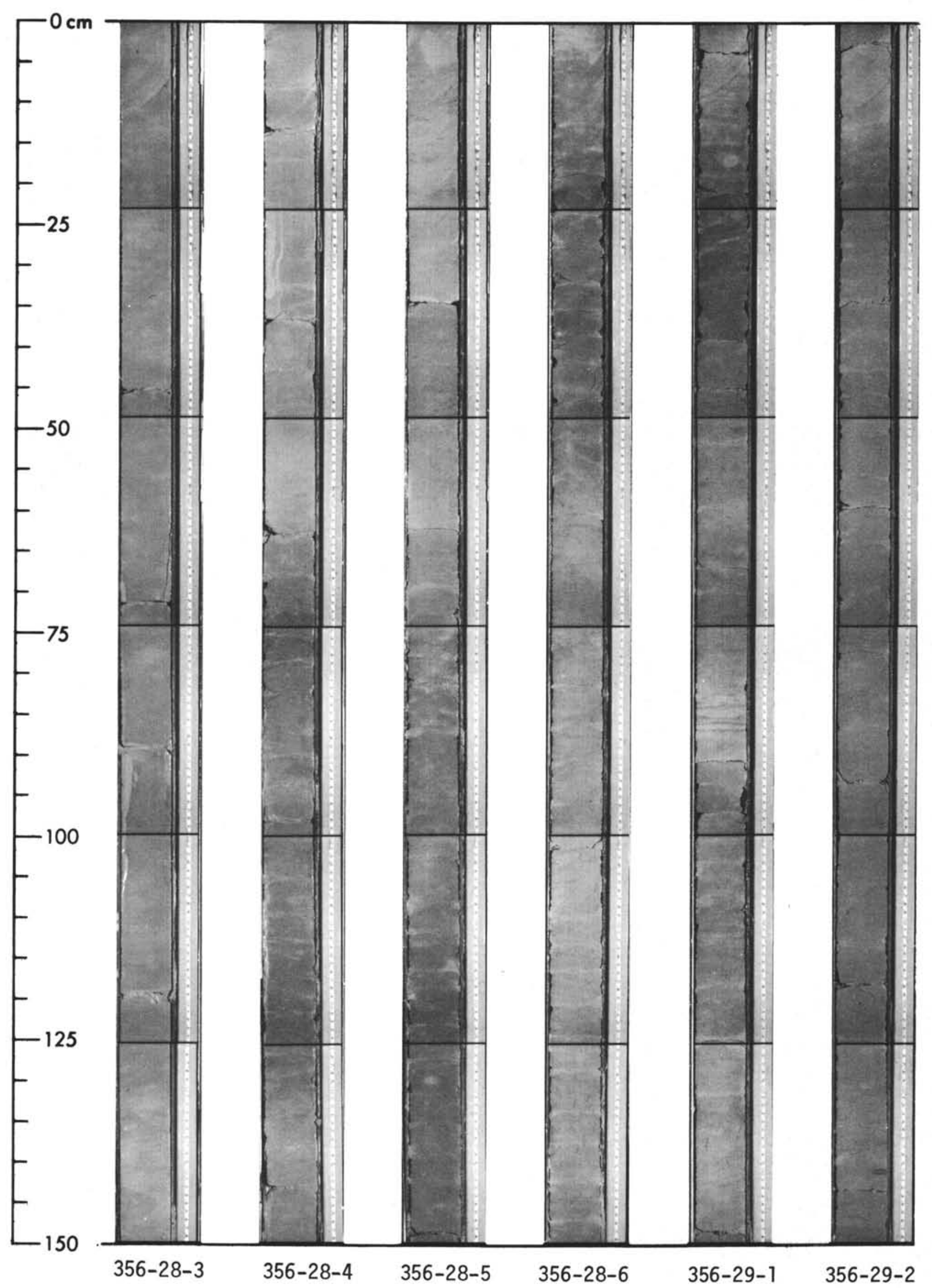




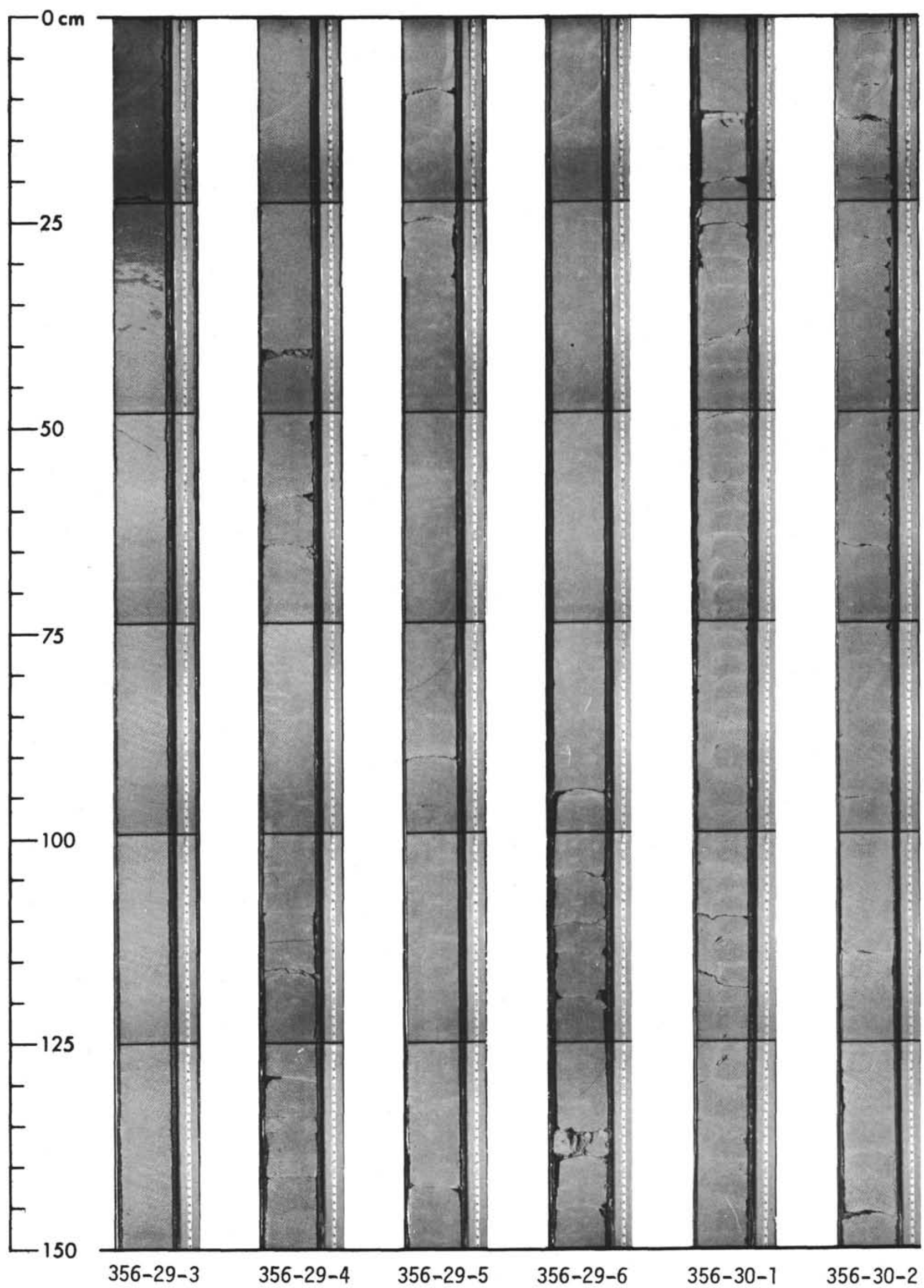




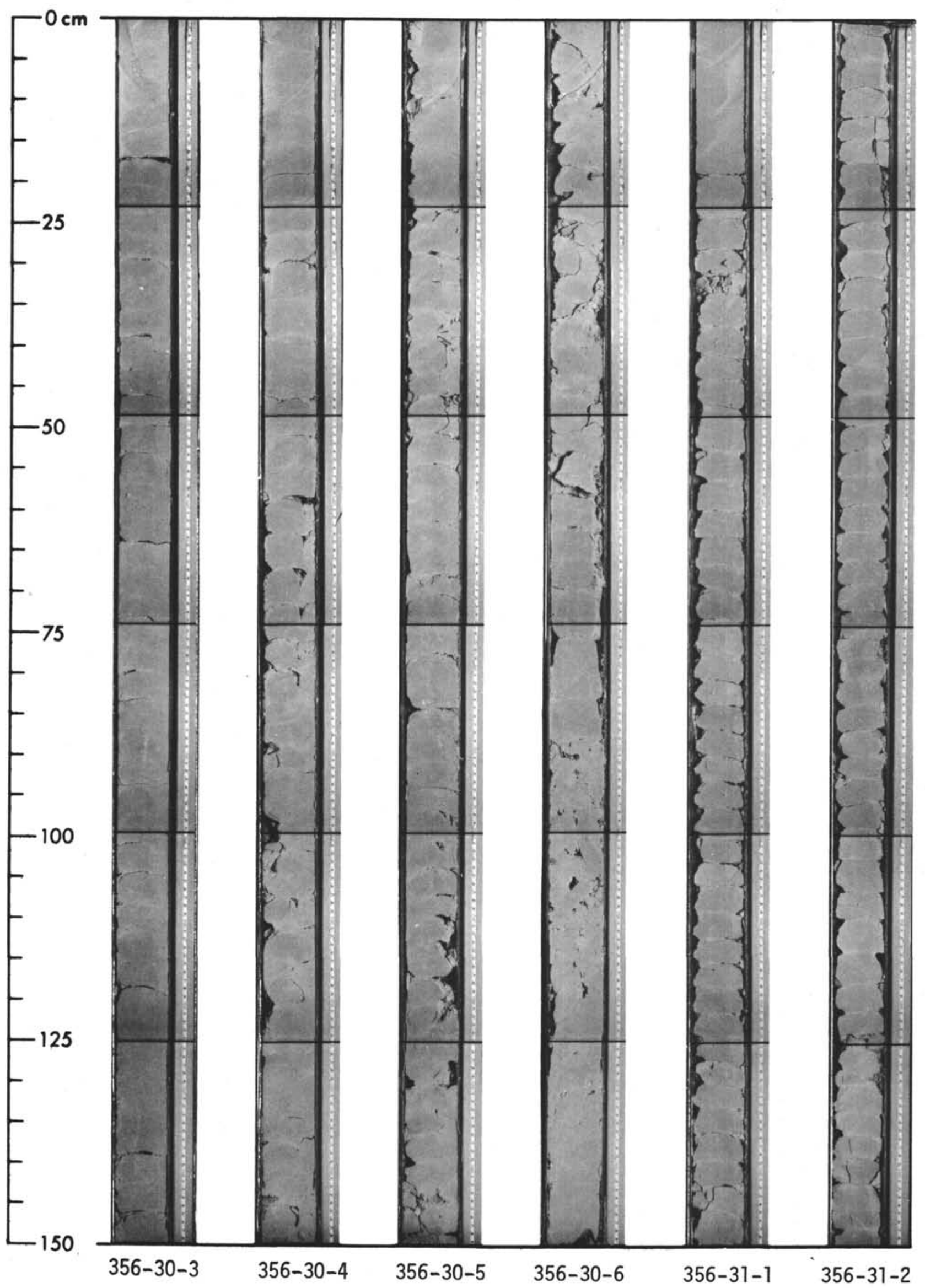


SITE 356: SÃO PAULO PLATEAU

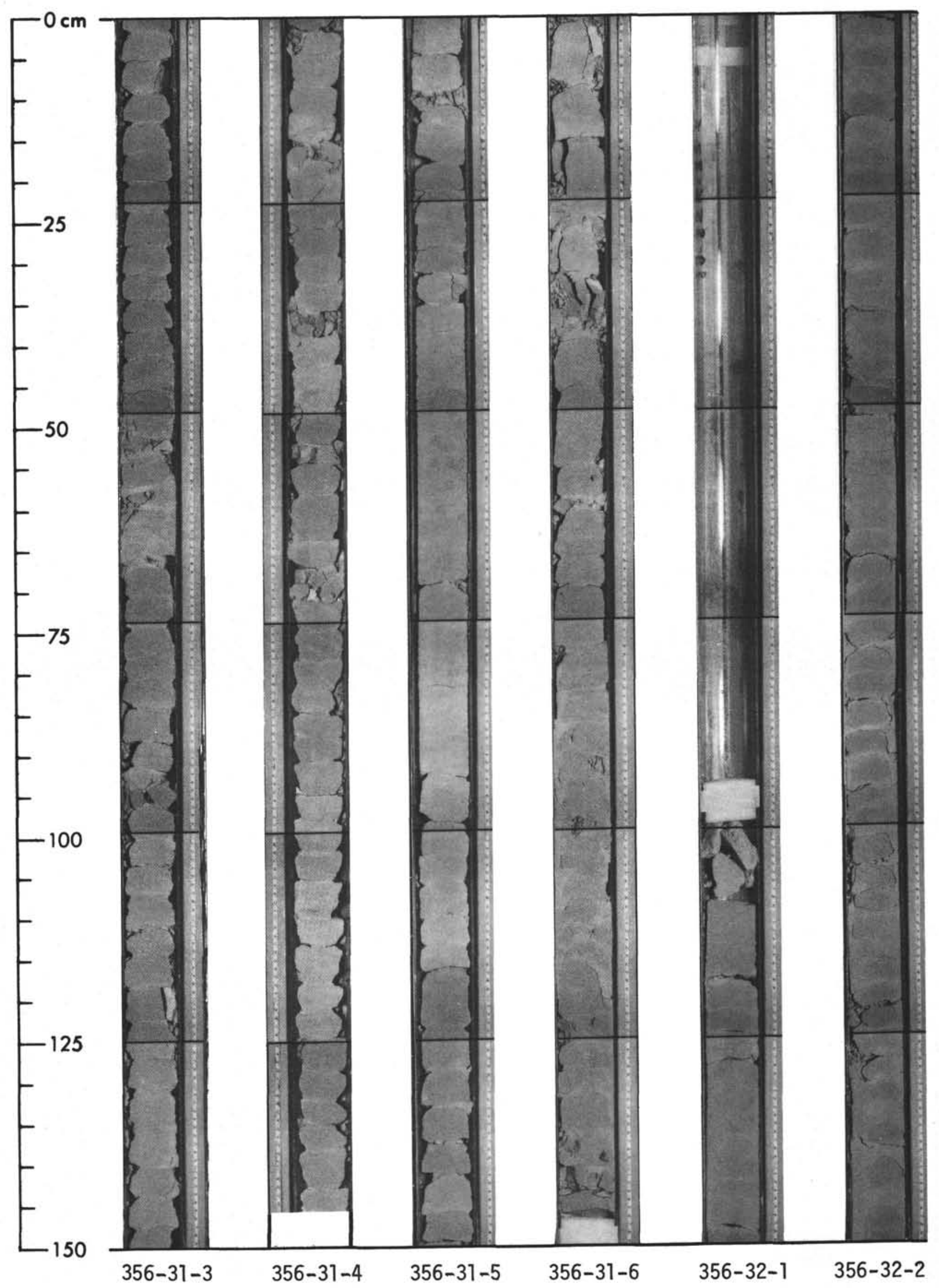


SITE 356: SÃO PAULO PLATEAU

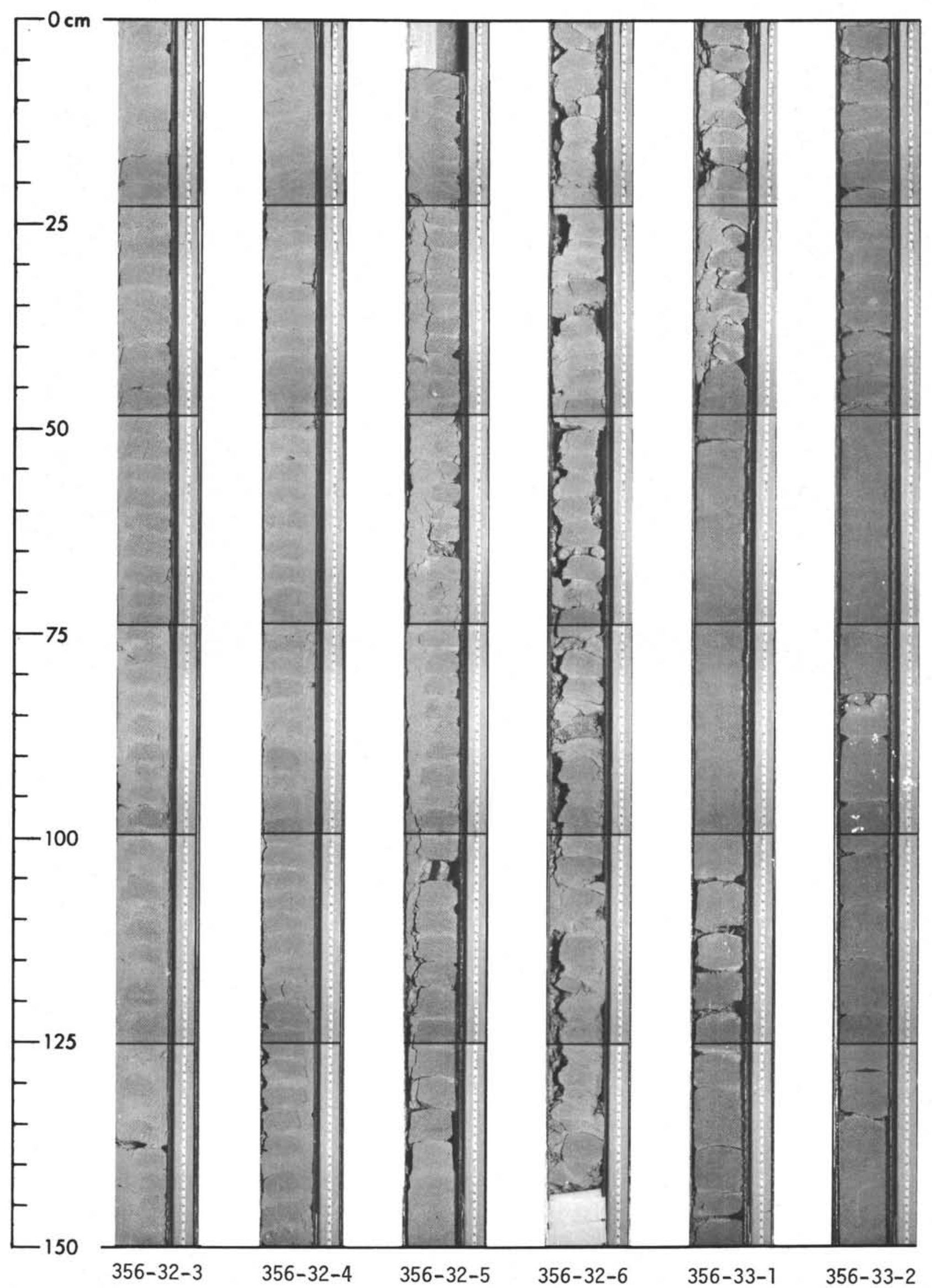


SITE 356: SÃO PAULO PLATEAU

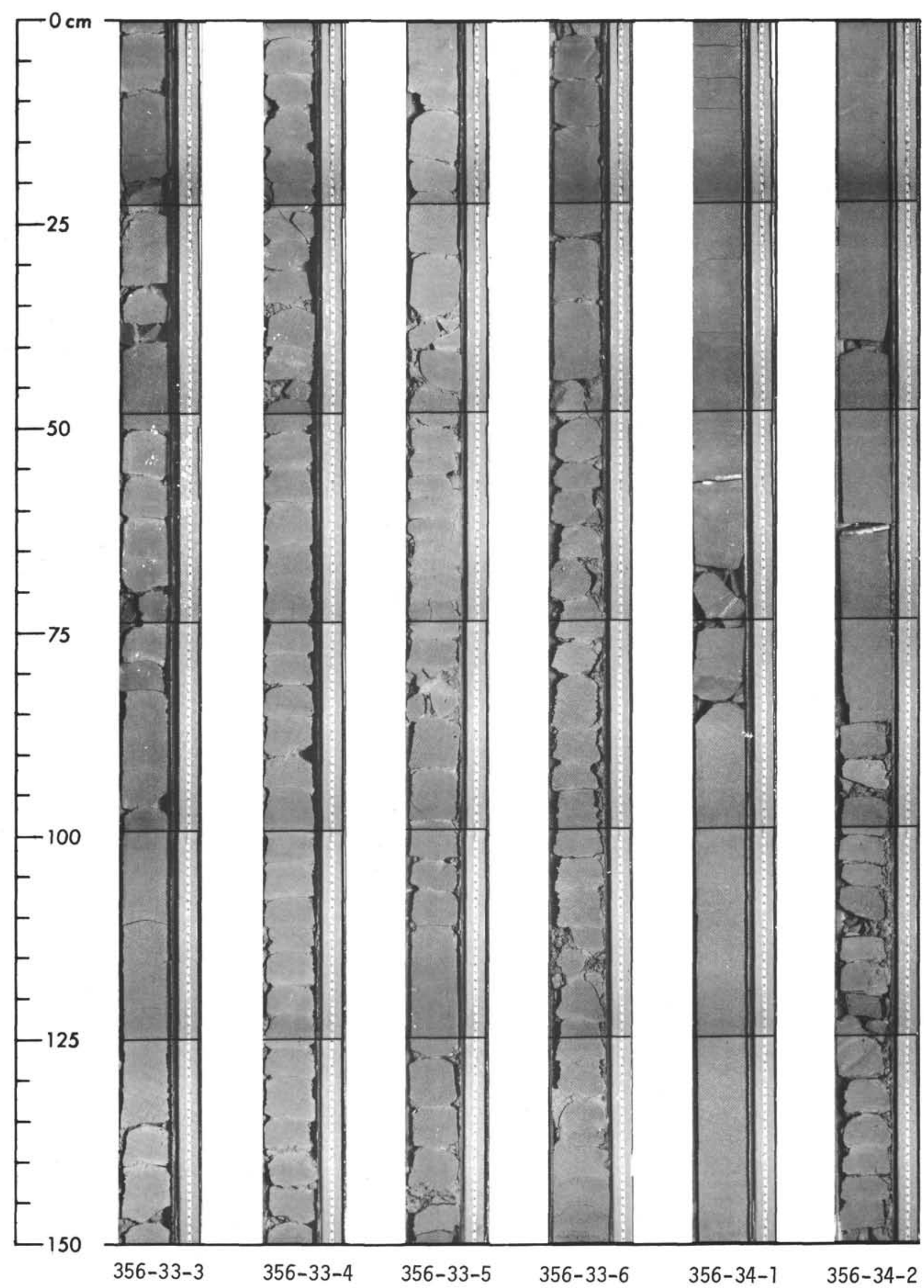


SITE 356: SÃO PAULO PLATEAU

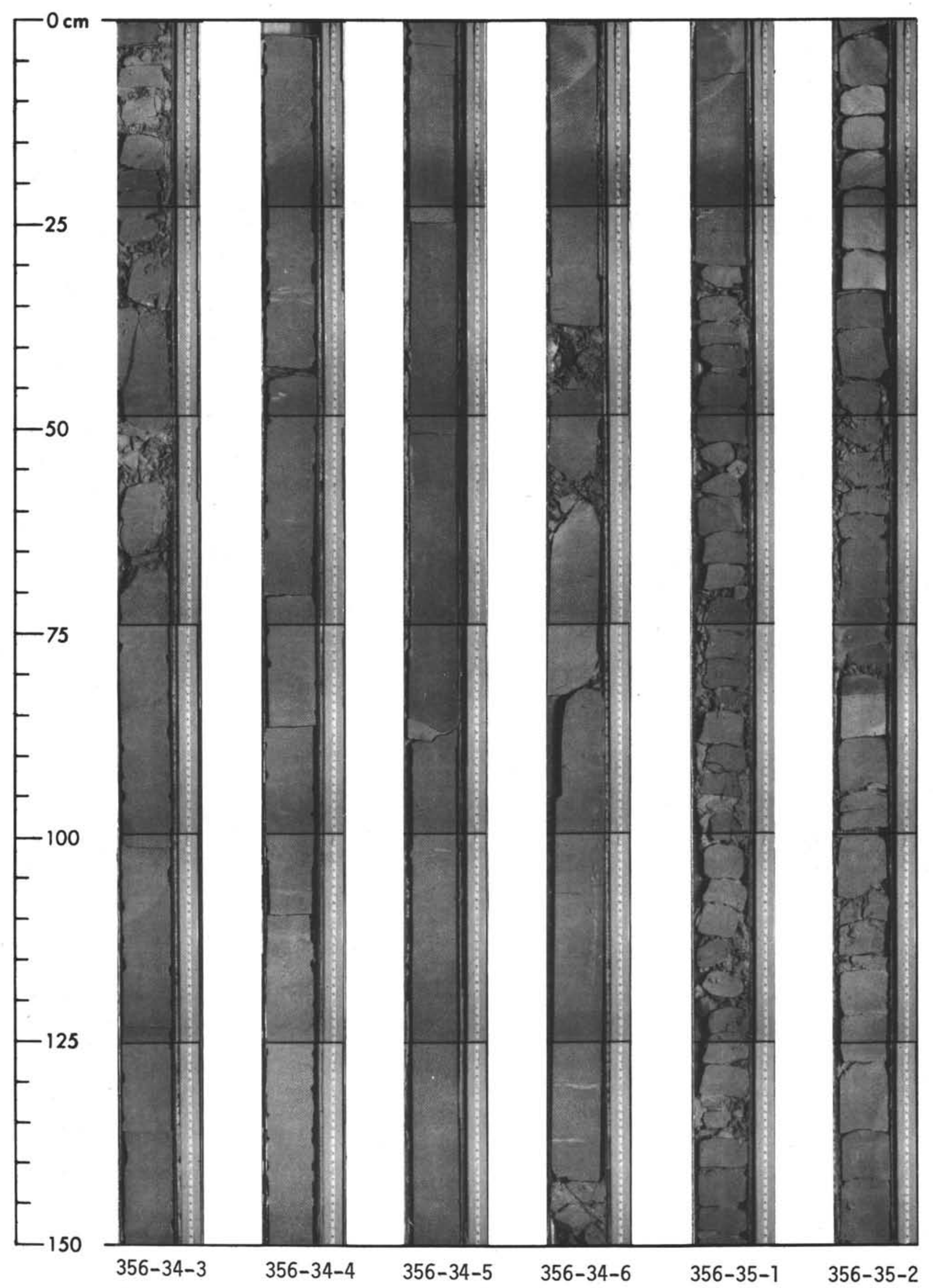


SITE 356: SÃO PAULO PLATEAU

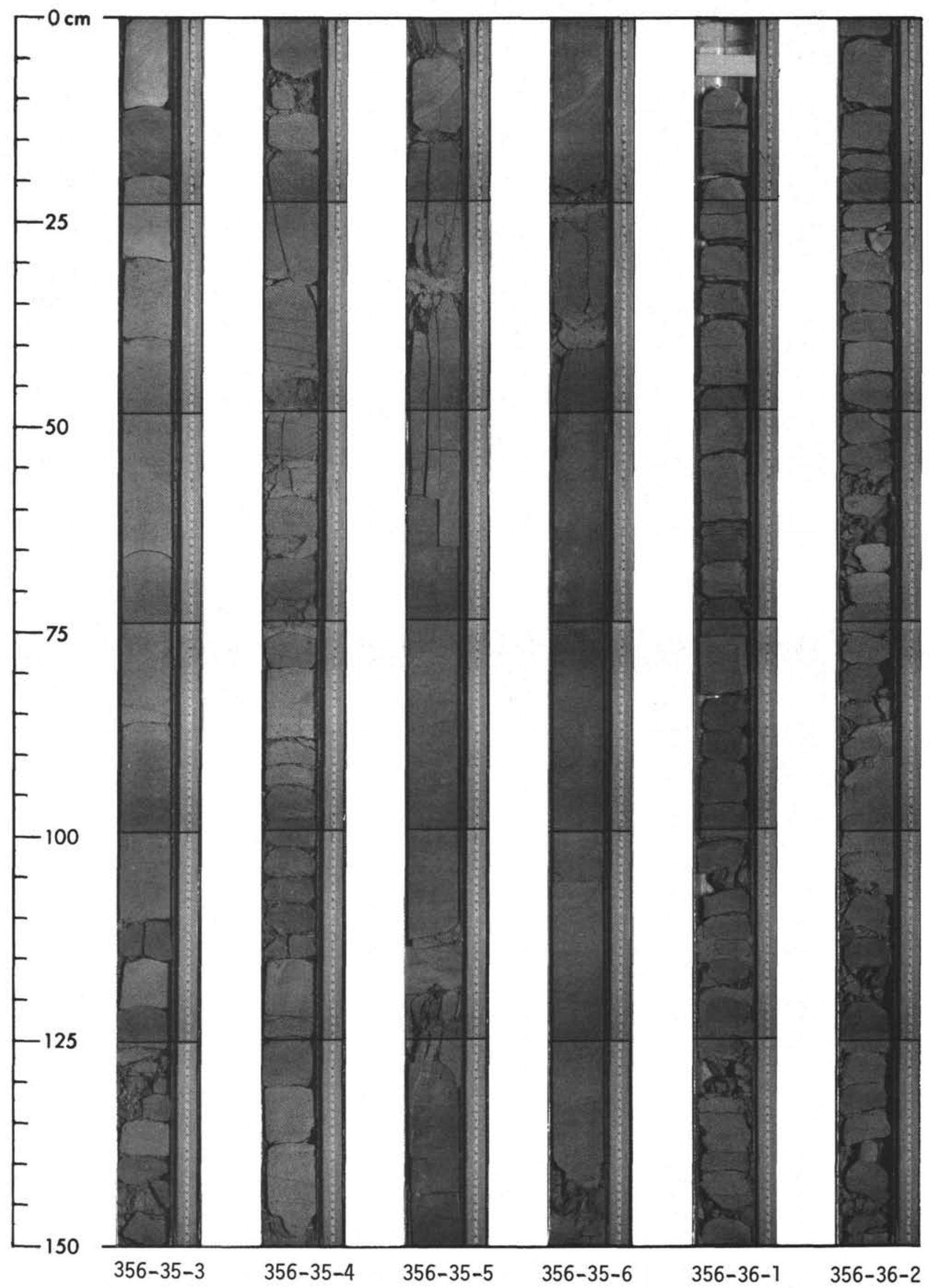




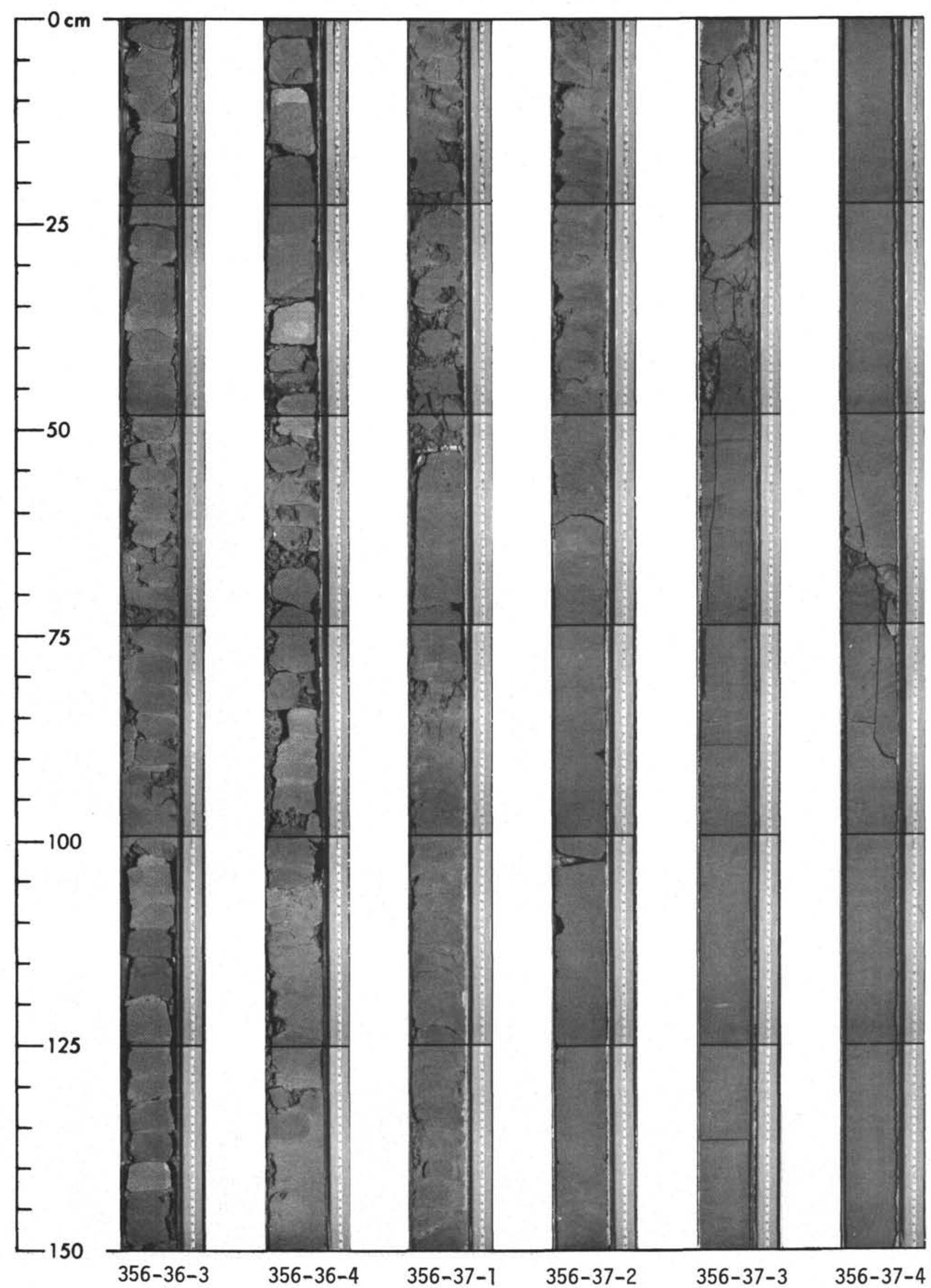


SITE 356: SÃO PAULO PLATEAU

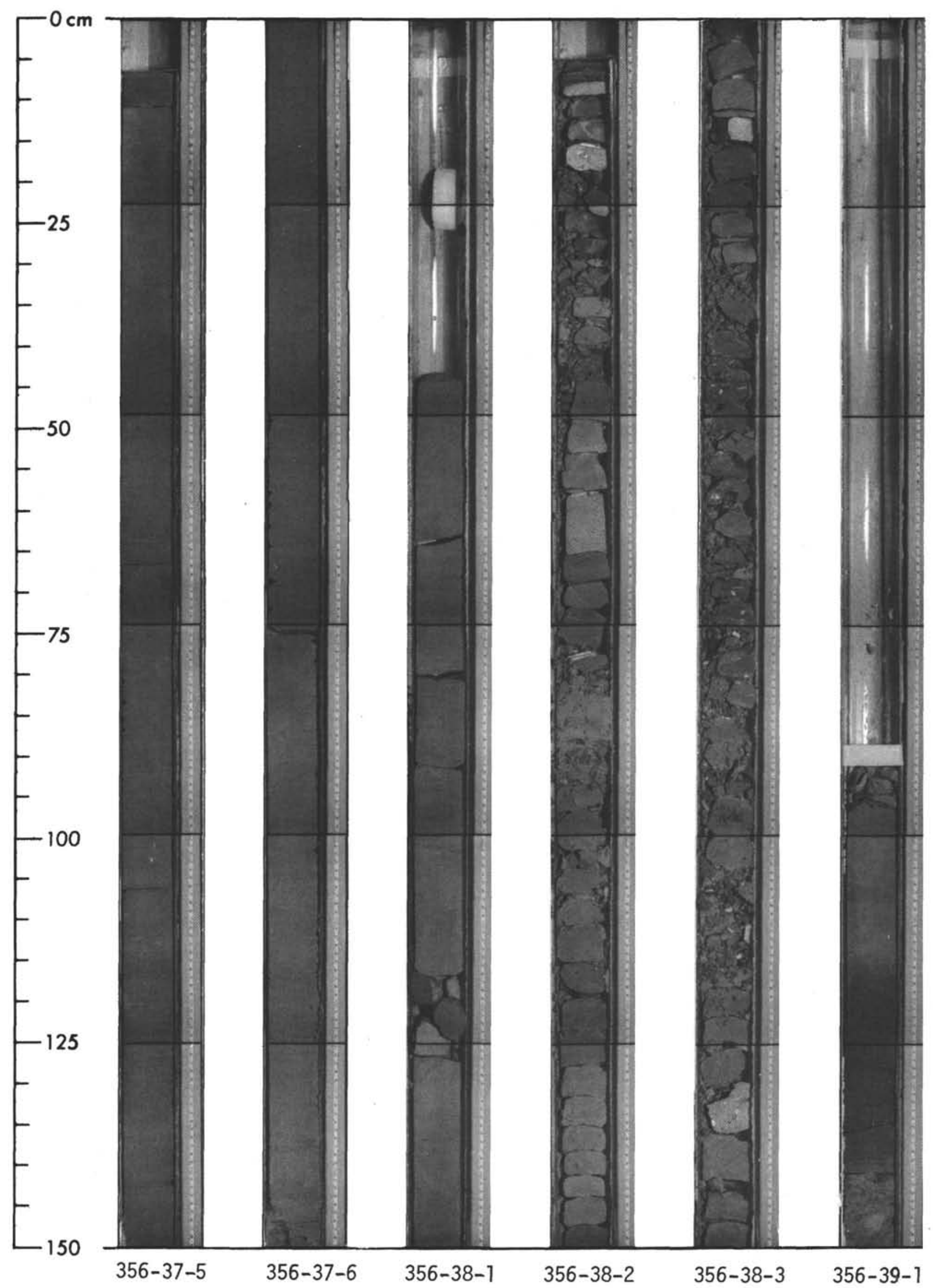


SITE 356: SATO PAULO PLATEAU

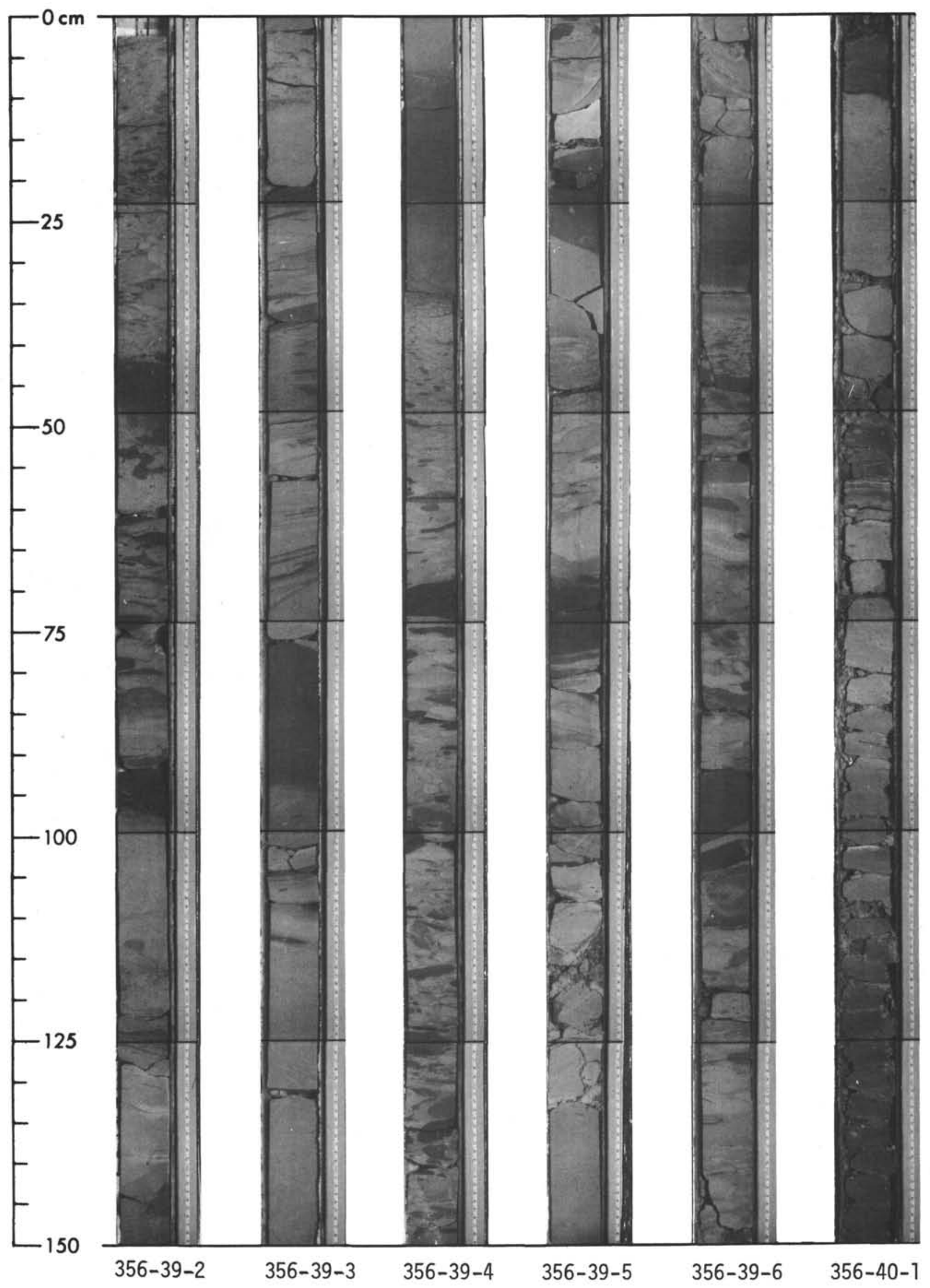




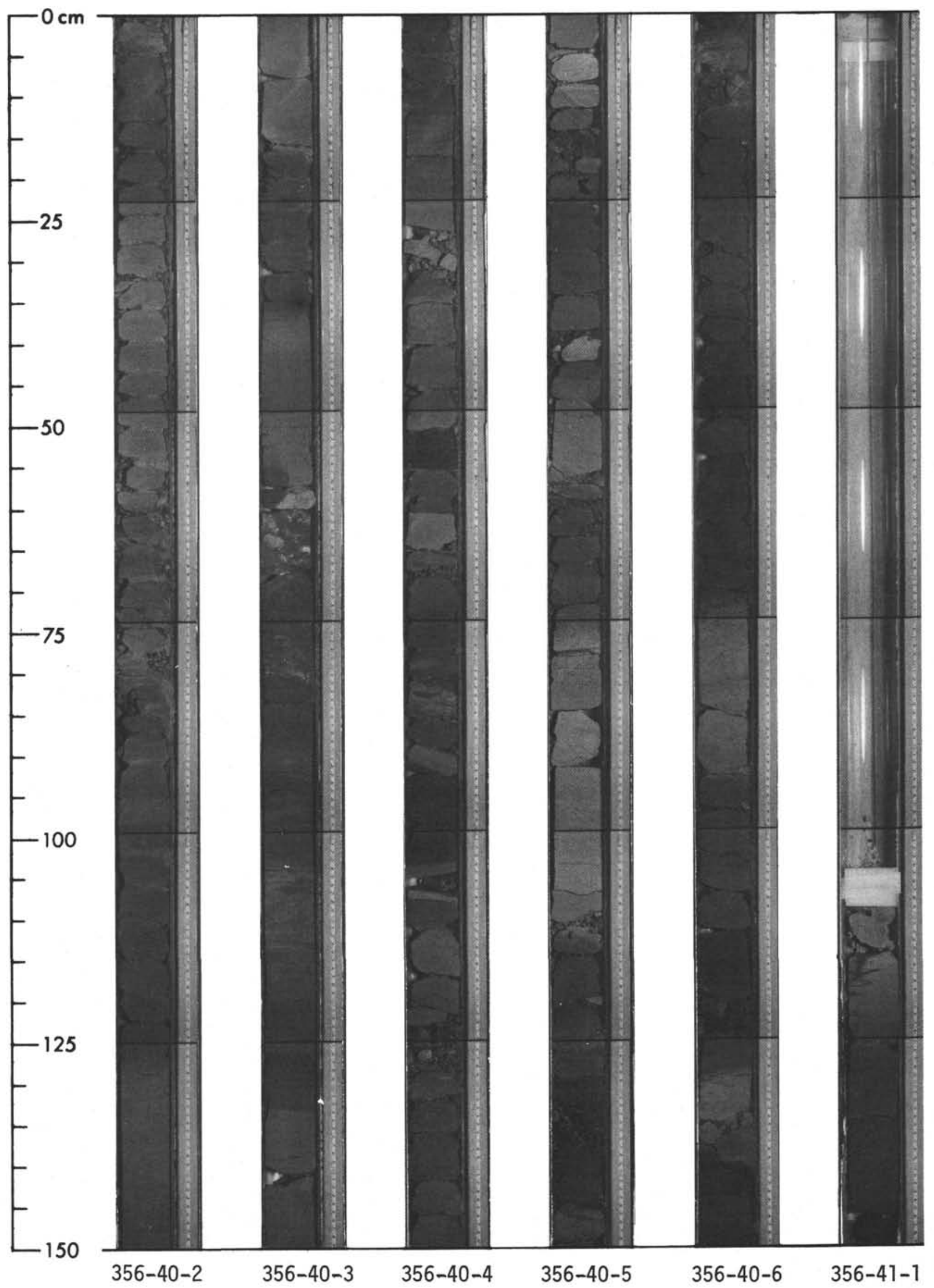




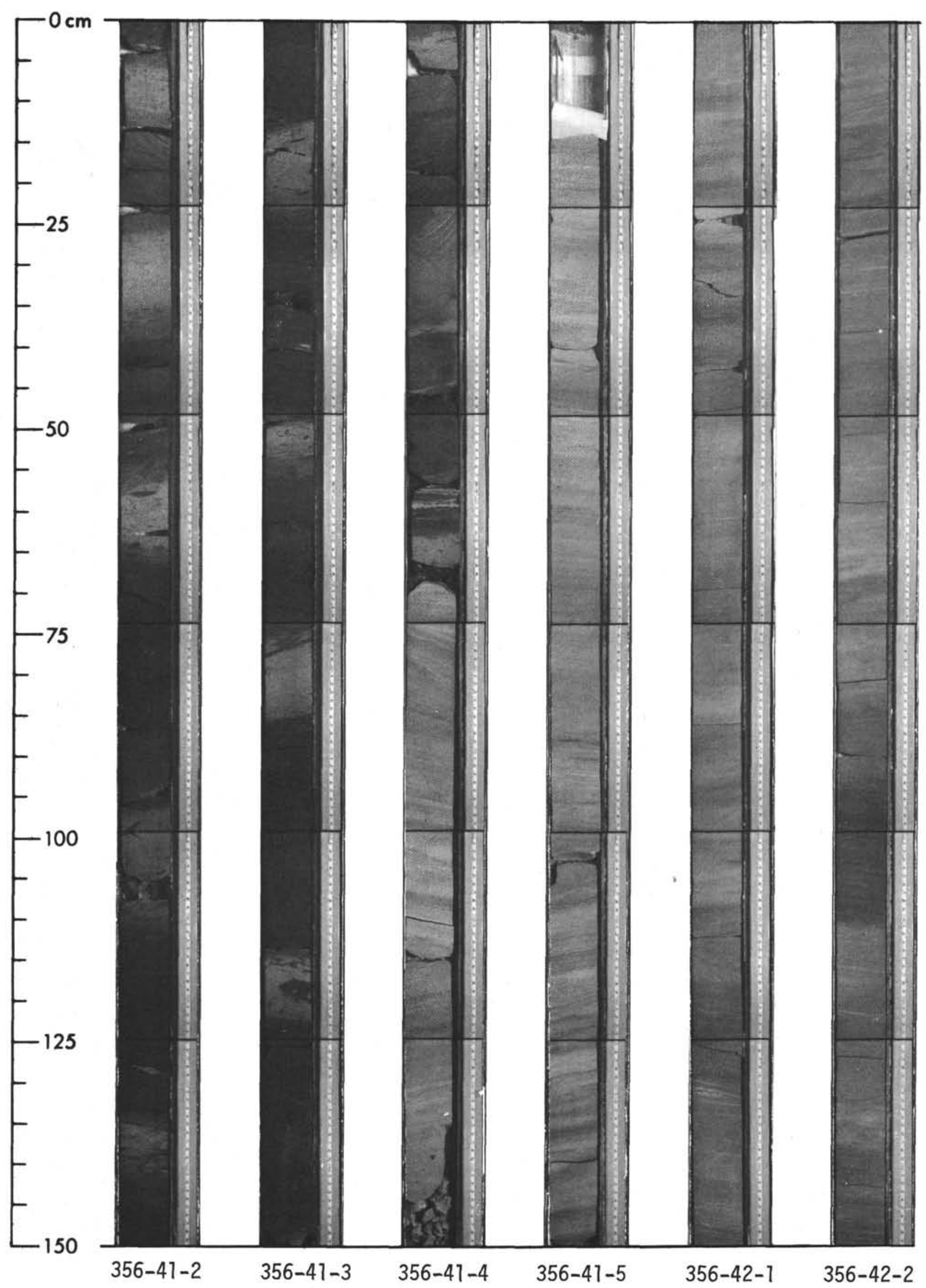


SITE 356: SÃO PAULO PLATEAU

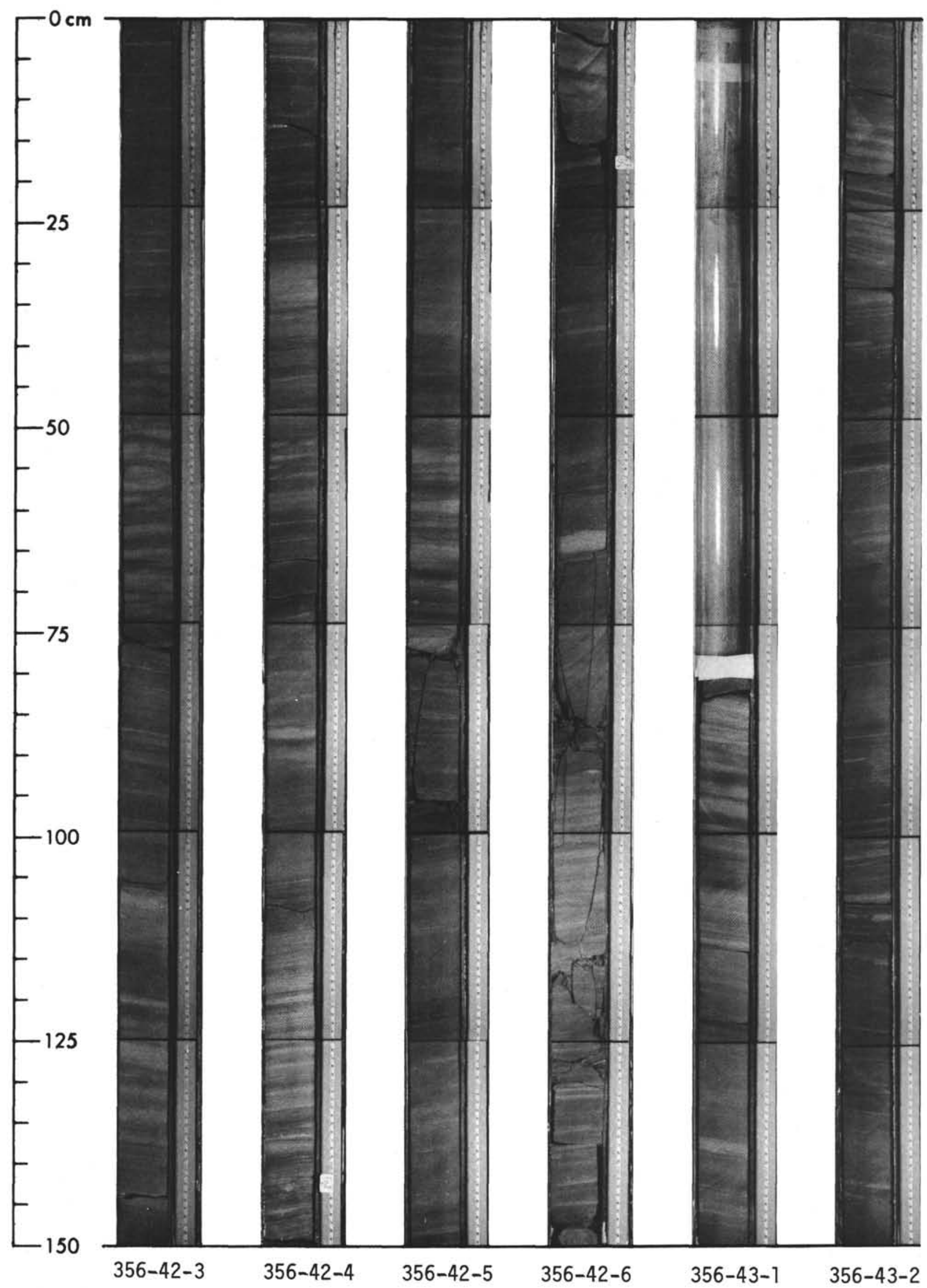


SITE 356: SÃO PAULO PLATEAU

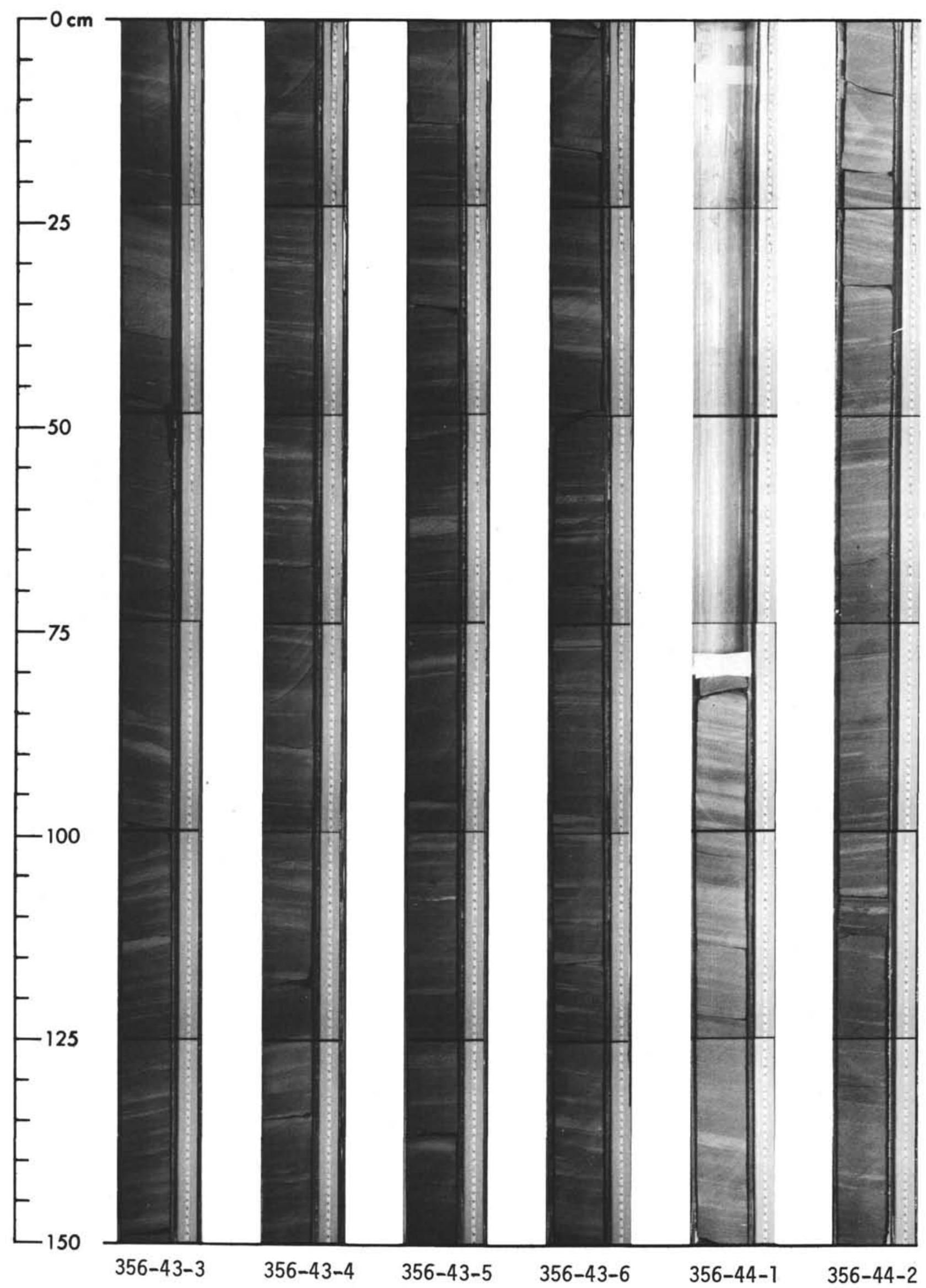


SITE 356: SÃO PAULO PLATEAU

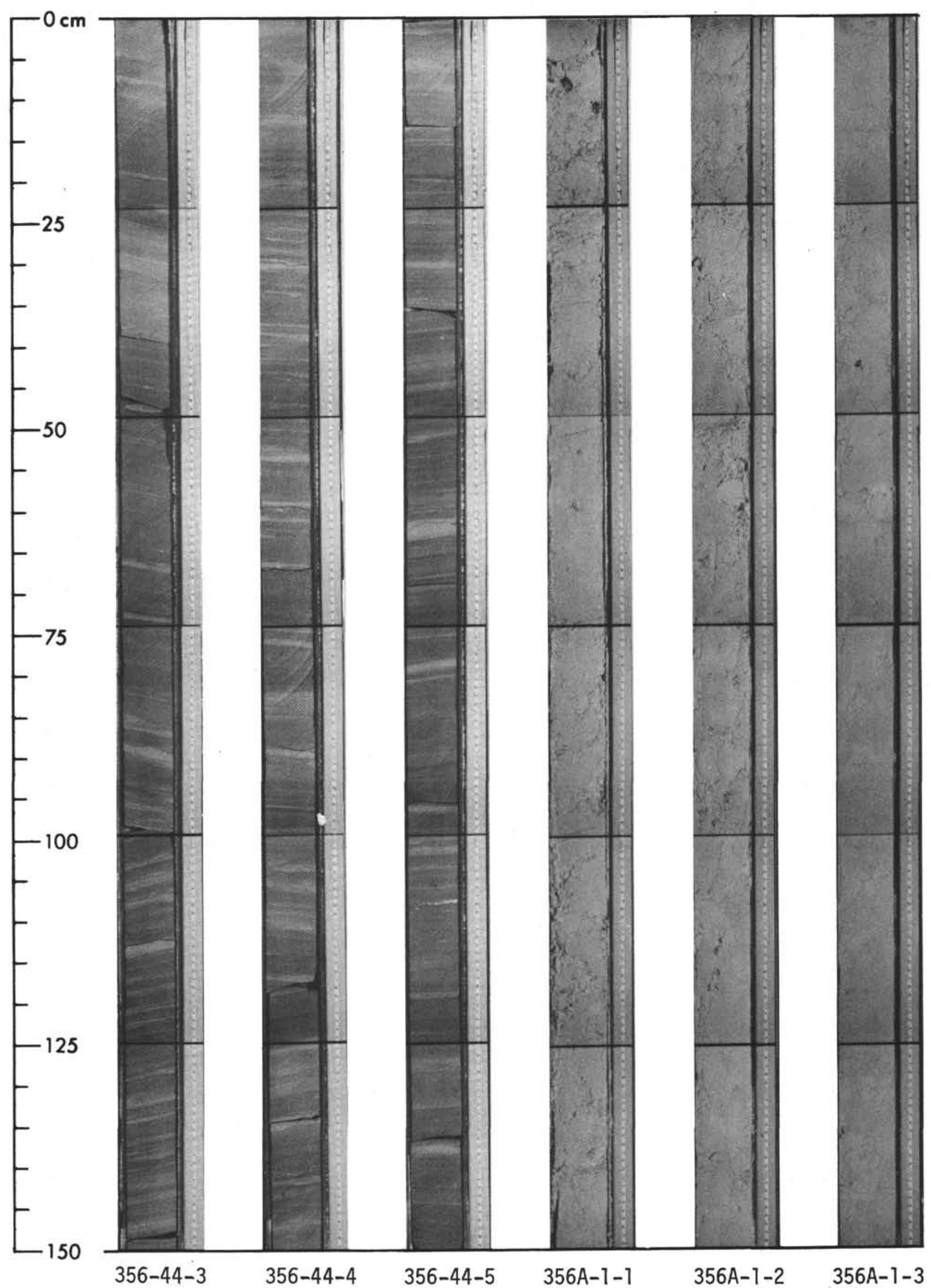




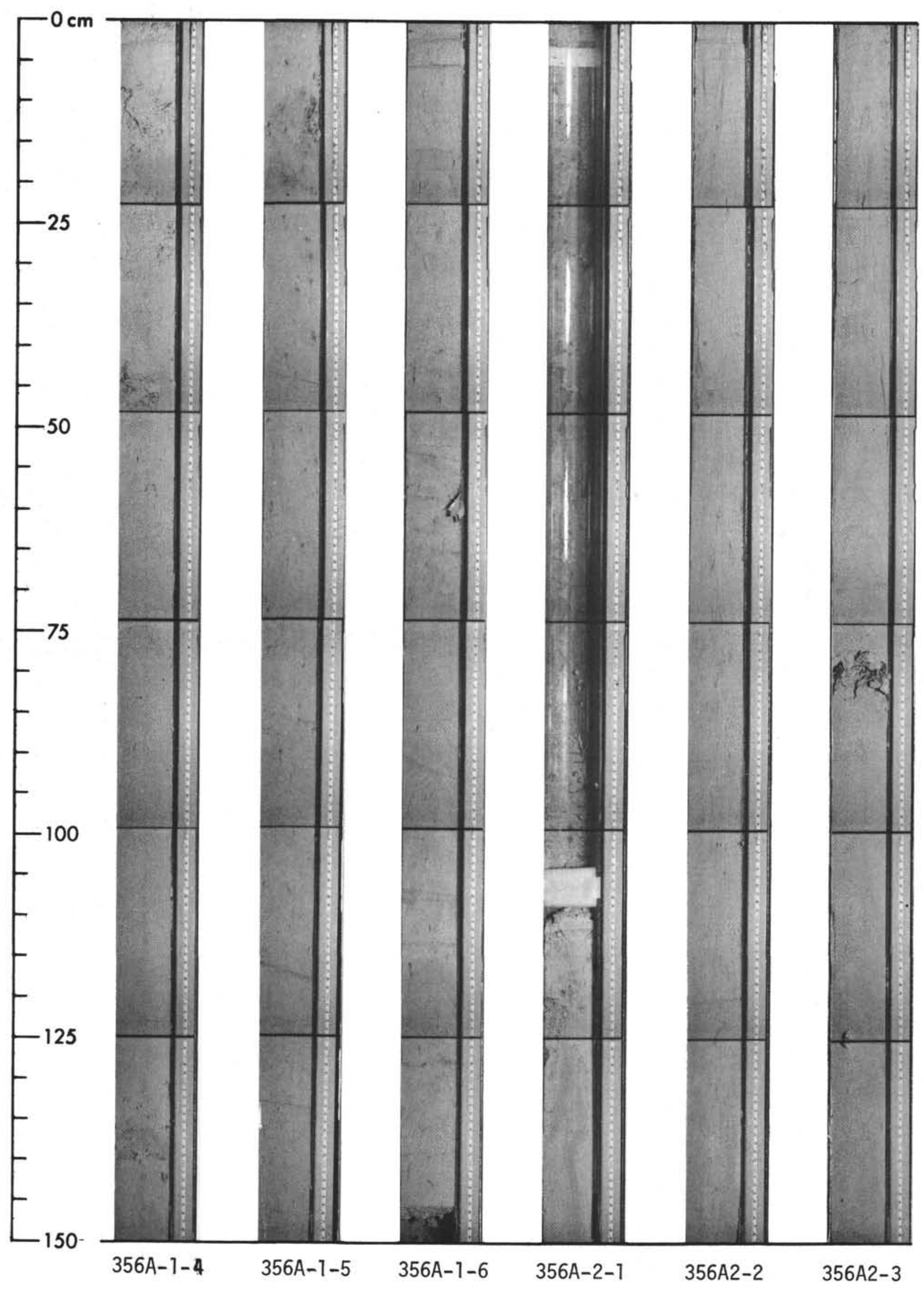


SITE 356: SÃO PAULO PLATEAU

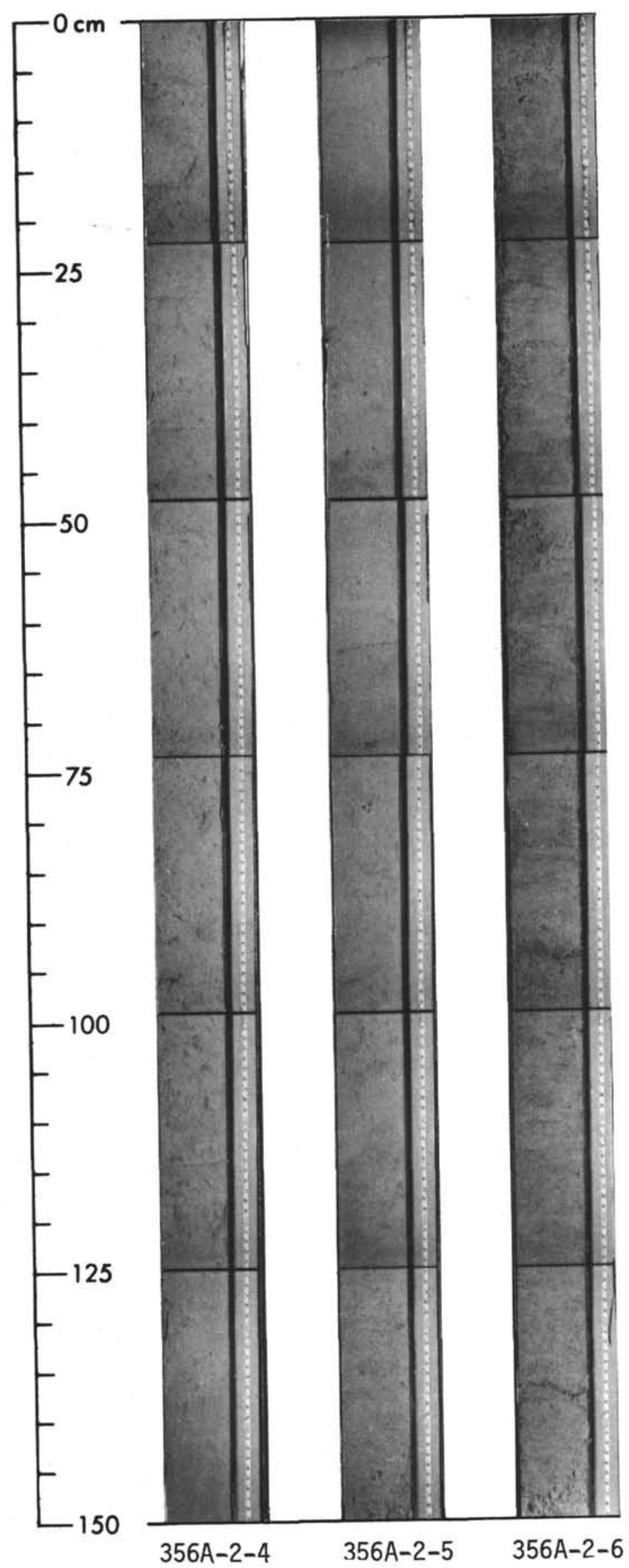

